\title{
Relationship between Fidelity and Dose of Human Patient Simulation, Critical Thinking Skills, and Knowledge in an Associate Degree Nursing Program
}

\author{
Rosella I. Beebe \\ West Virginia University
}

Follow this and additional works at: https://researchrepository.wvu.edu/etd

\section{Recommended Citation}

Beebe, Rosella I., "Relationship between Fidelity and Dose of Human Patient Simulation, Critical Thinking Skills, and Knowledge in an Associate Degree Nursing Program" (2012). Graduate Theses, Dissertations, and Problem Reports. 4831.

https://researchrepository.wvu.edu/etd/4831

This Dissertation is protected by copyright and/or related rights. It has been brought to you by the The Research Repository @ WVU with permission from the rights-holder(s). You are free to use this Dissertation in any way that is permitted by the copyright and related rights legislation that applies to your use. For other uses you must obtain permission from the rights-holder(s) directly, unless additional rights are indicated by a Creative Commons license in the record and/ or on the work itself. This Dissertation has been accepted for inclusion in WVU Graduate Theses, Dissertations, and Problem Reports collection by an authorized administrator of The Research Repository @ WVU.

For more information, please contact researchrepository@mail.wvu.edu. 
Relationship between Fidelity and Dose of Human Patient Simulation, Critical Thinking Skills, and Knowledge in an Associate Degree Nursing Program

Rosella I. Beebe

\author{
Dissertation submitted to the \\ College of Human Resources and Education \\ at West Virginia University \\ in partial fulfillment of the requirements \\ for the degree of
}

Doctor of Education

in

Educational Leadership

\author{
Rick Breault, Ed.D., Co-chair \\ Ernest Goeres, Ph.D., Co-chair \\ Reagan Curtis, Ph.D. \\ Cynthia A. Persily, Ph.D. \\ Lisa Straight, DNP \\ Department of Educational Leadership
}

Morgantown, West Virginia

2012

Keywords: Nursing Education, Traditional Clinical, Human Patient Simulation, Critical Thinking, Knowledge

Copyright 2012 Rosella I. Beebe 


\begin{abstract}
Relationship between Fidelity and Dose of Human Patient Simulation, Critical Thinking Skills, and Knowledge in an Associate Degree Nursing Program
\end{abstract}

Rosella I. Beebe

This study examined the relationship between human patient simulation (HPS), critical thinking skills, and knowledge acquisition after HPS was integrated across the curriculum of an associate degree nursing program to determine if differences existed in critical thinking and knowledge of students based on the fidelity of HPS used and amount of student exposure to HPS. The effectiveness of HPS when used as a teaching strategy in lieu of traditional clinical experiences was examined to determine the impact on critical thinking and knowledge in relation to the percentage of traditional clinical experiences substituted with HPS. The National League for Nurses $^{e(}$ (NLN) Nursing Education Simulation Framework, based on Kolb ${ }^{e c}$ s experiential learning theory, provided the framework. An ex post facto design was used with a convenience sample of 187 graduates drawn from the six cohorts of graduates who graduated from the nursing program spring 2006 through spring 2010. The Health Education Systems Incorporated (HESI) exit exam ${ }^{\text {ee }}\left(\mathrm{E}^{2}\right)$ composite score and HESI $E^{2}$ critical thinking (CT) subscore, used to measure nursing knowledge and critical thinking skills, were compared among the six cohorts of graduates exposed to varying degrees of fidelity and number of hours of traditional clinical experiences substituted with HPS during their educational program. Analysis of variance revealed that a significant difference in knowledge $(p=0.012)$ existed between the six cohorts. In addition, analysis of covariance revealed that a significant difference in critical thinking skills $(p=0.003)$ existed between the six cohorts. Polynomial contrasts identified a significant, positive linear trend in HESI $E^{2}$ mean scores $(p=.001)$ and HESI E $E^{2}$ T mean scores $(p<.001)$ across the six cohorts as exposure to and number of traditional clinical hours substituted with simulation increased. Significant statistical differences existing between pairs of cohorts were identified by pairwise comparisons of the six cohorts. The results supported the use of HPS as an effective teaching strategy in lieu of a small percentage of traditional clinical experiences. No significant statistical differences were identified in knowledge and critical thinking based on the fidelity of HPS used. 


\section{Acknowledgments}

I owe my gratitude to a great number of people who have made this dissertation possible. My genuine respect and deepest gratitude is to my advisor and co-chair of my dissertation committee, Dr. Ernest Goeres. I would not have had the courage to finish this journey without his continuous support, encouragement, guidance, and assistance over these past years. His spirit of adventure and sense of humor, excitement in regards to teaching, knack at giving practical advice, and willingness to offer guidance from the onset to the very end of my graduate work have helped to make these past five years bearable. His faith in my intellectual capacity and belief in my ability gave me the confidence I needed to move forward and complete this dissertation. My deepest appreciation for his willingness to "stay on" and co-chair my dissertation committee, despite his attempts to retire. His friendship will be cherished forever.

I am also indebted to Dr. Rick Breault who, despite being new to the institution, volunteered to step in as co-chair of my dissertation committee when all other faculty of the department had left. Without his help and guidance, this dissertation would not have been possible. I am deeply grateful for the insightful discussions that helped me sort out the direction for my research. His support, guidance, and encouragement as we walked together through the dissertation process is sincerely appreciated and valued.

A special thank you to each of the members of my dissertation committee. My dissertation would never have been started without the assistance of Dr. Cynthia Persily who listened to my ideas and helped me narrow my focus to discover my topic. The statistical guidance of Dr. Regan Curtis in identifying the statistical models to utilize confirmed that my research was possible. I am appreciative of his assistance in refreshing my knowledge of statistics and his guidance throughout the statistical analysis of this research. To my friend and 
colleague, Dr. Lisa Straight, I will always be grateful for her moral support, encouragement, and dedication as she stood by me to ensure my completion of this dissertation. My sincere appreciation for her ability to be straight forward with me, the countless hours she spent reading and proofreading my writings, and the time she spent listening to me talk, rant, and rave about my frustrations.

I would like to acknowledge and express my gratitude to several individuals for their contributions in helping me complete this dissertation. A special thank you to Lisa Gianettino for her efforts in assisting me in obtaining many books, articles, and resources for my literature review. I would also like to thank Dr. Patricia Gaston for her willingness to read my work and offer feedback on my writing. My sincere thanks to Amy Richards who entered the data and maintained all the student files for my database. To my friends and colleagues, particularly the faculty and staff of the Health Sciences Division at WVU Parkersburg, a special thank you for all of your help, support, and encouragement that have kept me sane throughout these difficult years. Your friendship is greatly valued.

Most importantly, I will always be grateful to Debbie Murphy who recognized the value of simulation in nursing education and was instrumental in the integration of human patient simulation across the nursing program of study. This research would not have been possible without her enthusiasm for technology and passion for teaching that gave her the drive and commitment to jump in head first and try human patient simulation. As a result, her efforts and success in implementing simulation have provided the background and data for this dissertation.

Completion of this journey would not have been possible without the love, support, and patience of both my immediate and extended family. I express my genuine thanks and appreciation for your tolerance of my moods, acceptance of my excuses, and understanding of 
my behaviors. A special acknowledgement for the loyalty, devotion, and warmth given by my four-legged grandchildren, Izzy and Chadwick, as they cuddled by me and watched me read, napped by my side, in my lap, or on the computer, or tolerated my piles of papers in their space on the floor. In addition, a special thank you to my husband Gary, for his love and support throughout our 32 years of marriage. Finally, I extend my sincere thanks and appreciation to my daughter Jessica, for her love and support that gave me the strength to keep moving forward. She knew when to rescue me from my chaos while pushing me to get started. Inspiring me with her own accomplishments, she was always there encouraging me not to quit and cheering me up when times became unbearable.

Five years ago, I embarked upon a journey that has proven to be rewarding and valuable in my growth as a professional, educator, and researcher. However, the emotions, feelings of frustrations, and hardships I have experienced are difficult to describe and ones that I hope are easier to forget. If it were not for my parents, who instilled in me my values, commitment, and work ethic, I would not have had the strength or drive to finish this journey I had started. Although they are not here today to celebrate my accomplishments, I dedicate this dissertation to them. With the encouragement, support, and assistance from many, this journey has come to an end. And for that, I am deeply grateful. 


\section{Table of Contents}

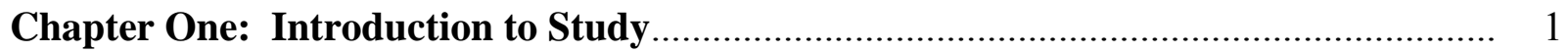

Support for HPS ......................................................................................... 2

Statement of the Problem........................................................................................... 5

Purpose and Research Questions............................................................................ 6

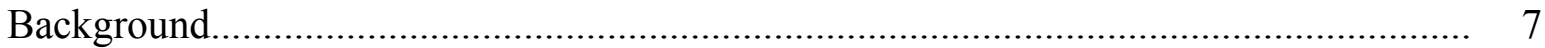

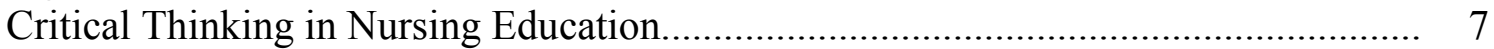

Experiential Learning through Clinical Experiences............................................. 9

Transitioning from Academia to Practice.......................................................... 11

The argument for the use of HPS in Nursing Educational Programs.......................... 13

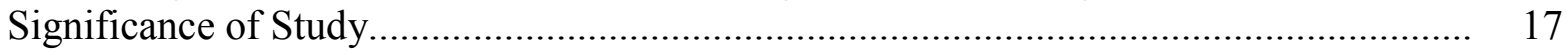

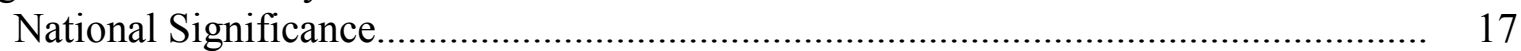

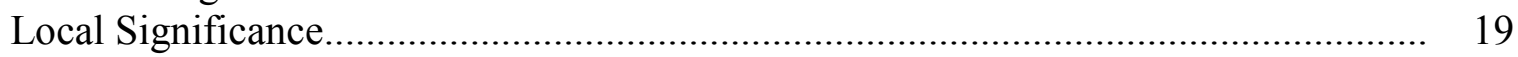

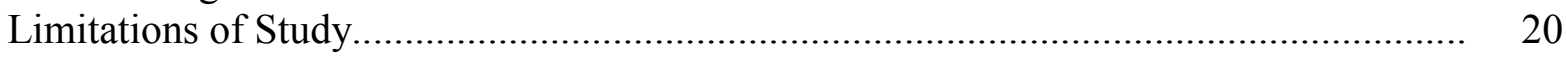

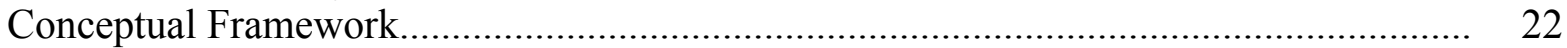

Critical Thinking and Surrogate Terms............................................................. 25

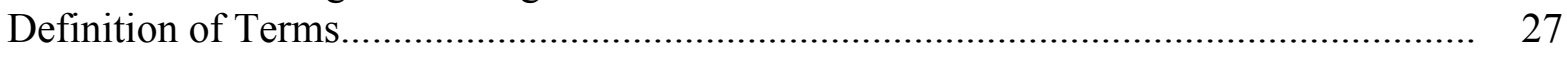

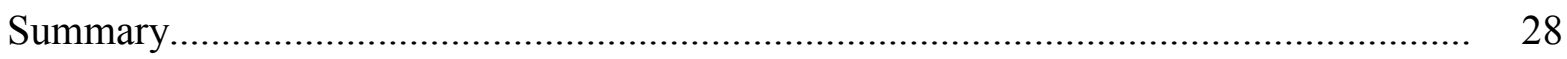

Chapter Two: Review of Literature ................................................................ 30

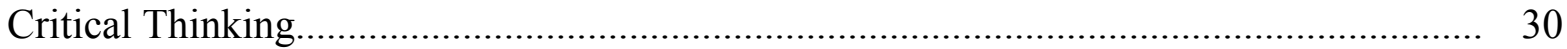

Critical Thinking and Relevance to Nursing............................................... 30

Critical Thinking Defined......................................................................... 32

Critical Thinking as Defined by Nursing...................................................... 37

Characteristics of the Critical Thinker................................................................ 39

Critical Thinking, Clinical Judgment, and Clinical Reasoning................................ 40

Learning and Acquisition of Knowledge................................................................ 41

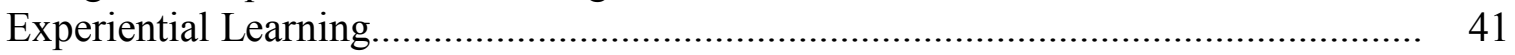

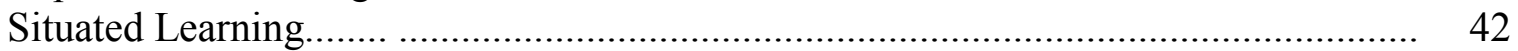

Experiential and Situated Learning as it Applies to Nursing..................................... 43

Reflective Thinking as it Relates to Critical Thinking and Knowledge......................... 44

Reflective Thinking Defined.......................................................................... 44

Reflective Thinking and Relevance to Nursing............................................... 45

Critical Thinking and Knowledge as Outcomes of Nursing Education.......................... 46

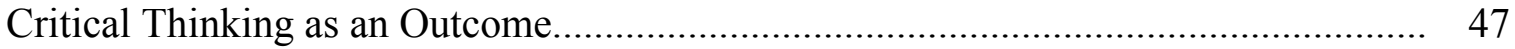

Critical Thinking and Development of Knowledge............................................... 47

Measurement of Knowledge and Critical Thinking............................................ 49

Development of Critical Thinking................................................................. 53

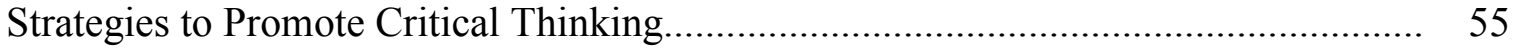

Nursing Education..................................................................................... 59

Traditional Clinical Education.................................................................... 60

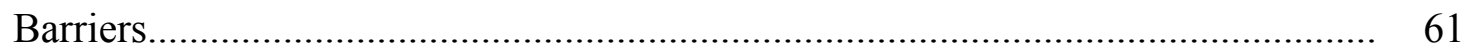

Strategies to Address Barriers....................................................................... 63

A Call for Change in Clinical Education.......................................................... 66

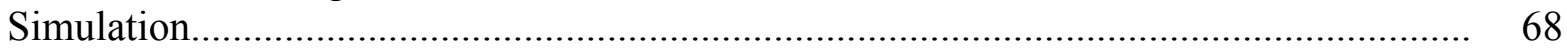


History of Simulation in Professional Education................................................ 68

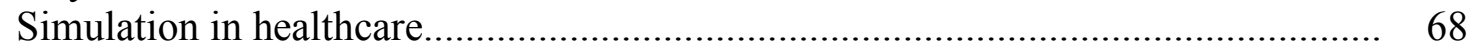

Research Related to HPS in medicine............................................................ 70

Evolution of Simulation in Nursing Education.................................................... 73

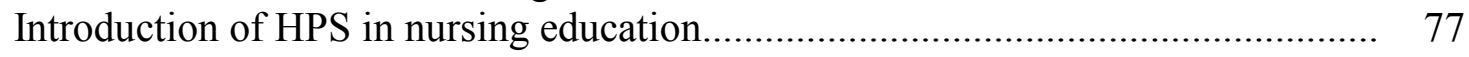

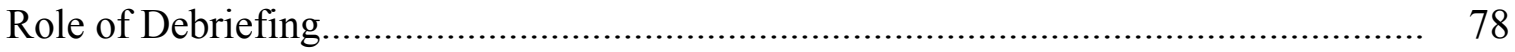

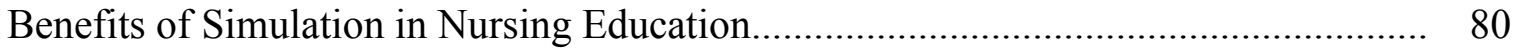

Research on Simulation in Nursing Education...................................................... 86

Low, Medium, and High-fidelity HPS in Nursing Education................................. 91

Student and Faculty Perceptions of Value and Satisfaction..................................... 98

Self-efficacy, Self-confidence, and Competency................................................... 103

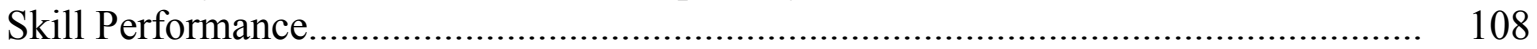

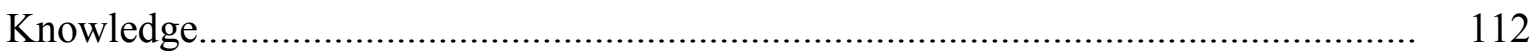

Critical Thinking.......................................................................................... 124

Critical Thinking and Knowledge.................................................................... 129

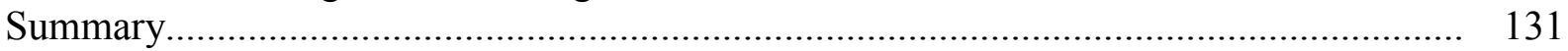

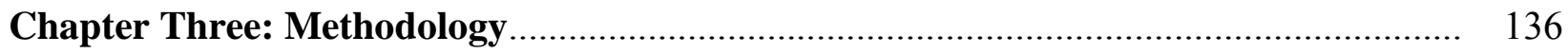

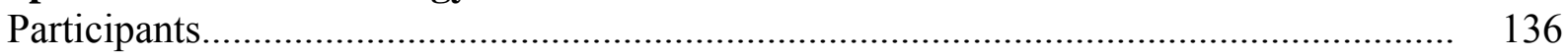

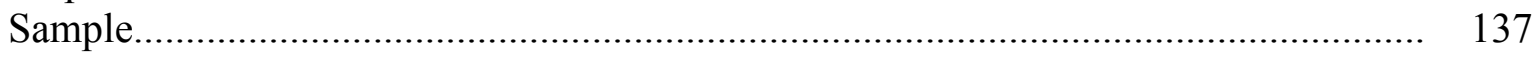

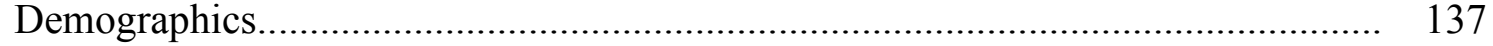

Academic Abilities on Admission.................................................................. 138

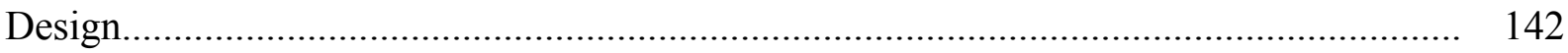

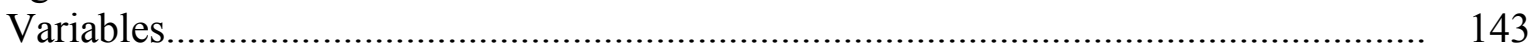

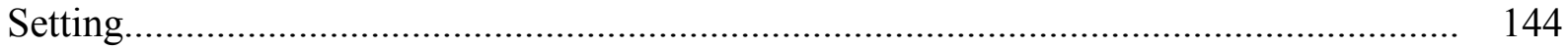

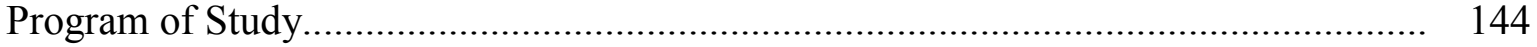

Simulation Laboratory and Simulators.......................................................... 145

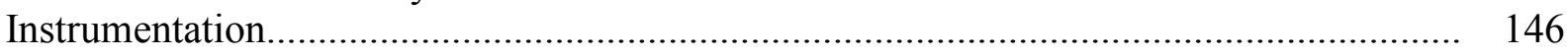

Description of the Instrument...................................................................... 147

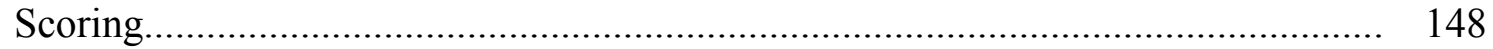

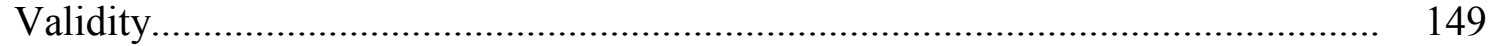

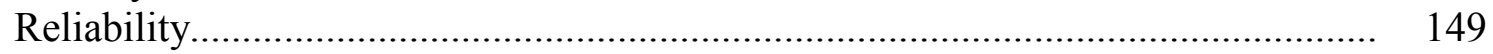

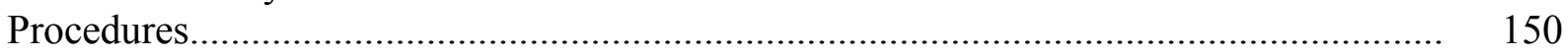

Introduction of Medium-Fidelity HPS............................................................ 150

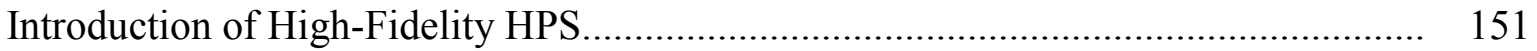

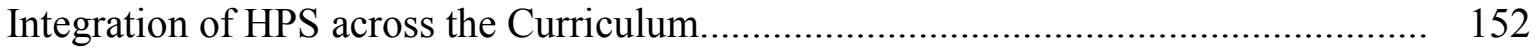

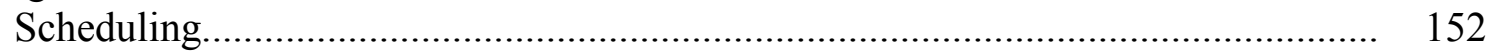

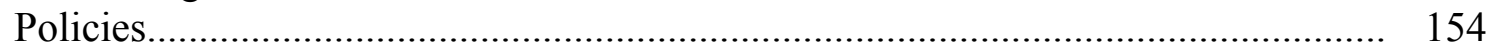

NLN ${ }^{e e}$ s Nursing Education Simulation Framework................................................. 154

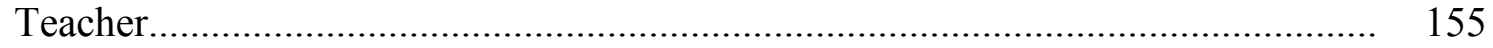

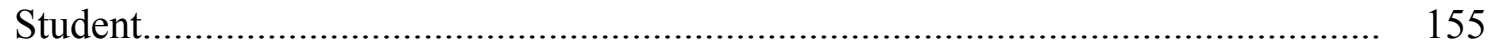

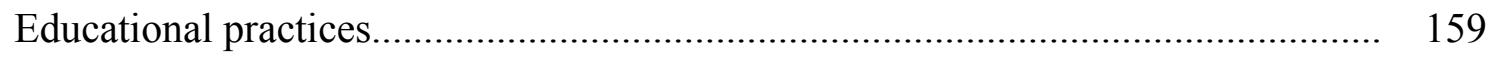

Simulation design characteristics.......................................................... 161

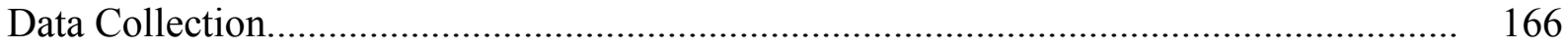

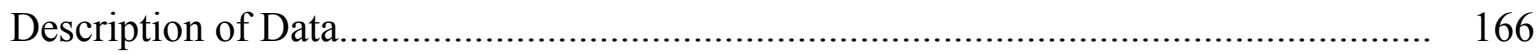




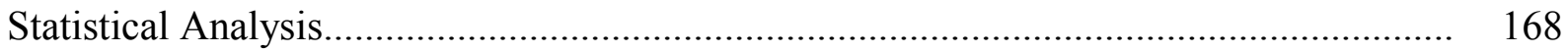

Violations of Assumptions................................................................................ 168

Research Questions 1 and 2........................................................................ 169

Research Questions 3 and 4........................................................................... 169

Polynomial Contrasts and Pairwise Comparisons.................................................... 170

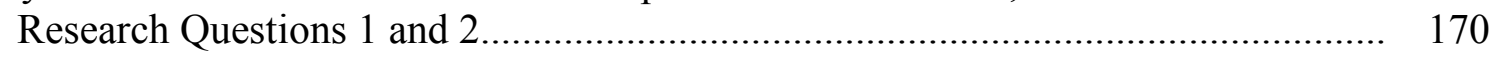

Research Questions 3 and 4..................................................................... 170

Analysis of Covariance.................................................................................. 171

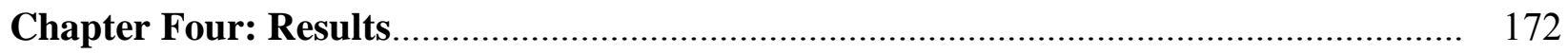

Analysis of Variance and Covariance...................................................................... 172

Tests for Assumptions.................................................................................... 173

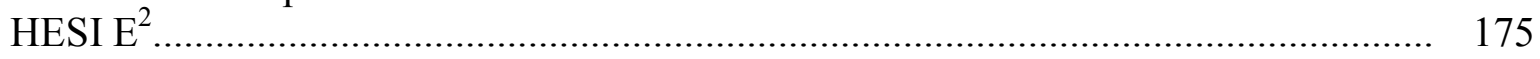

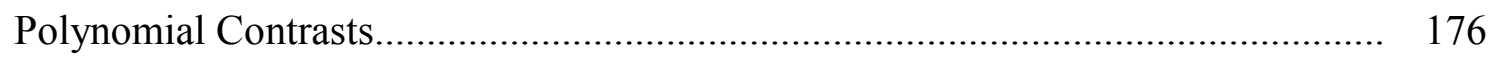

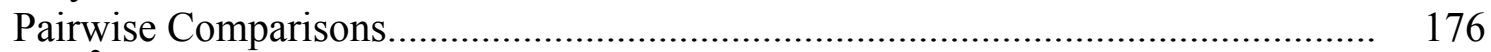

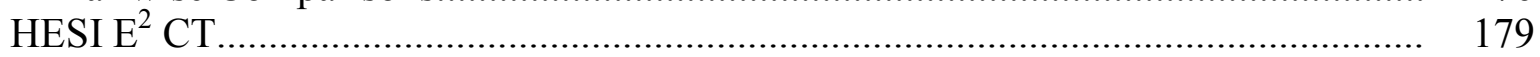

Analysis of Covariance............................................................................. 179

Polynomial Contrasts................................................................................. 180

Pairwise Comparisons....................................................................................... 181

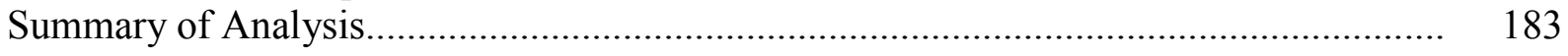

Chapter Five: Summary, Conclusions, and Recommendations.................................... 185

Review of the Problem........................................................................................... 185

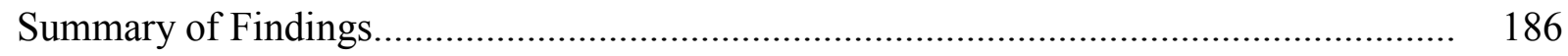

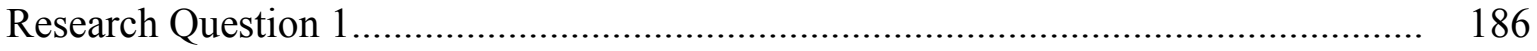

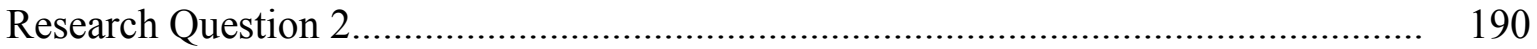

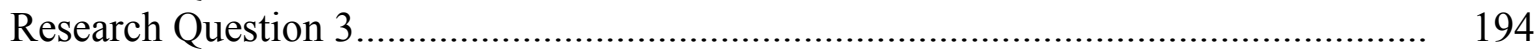

Research Question 4 .................................................................................. 198

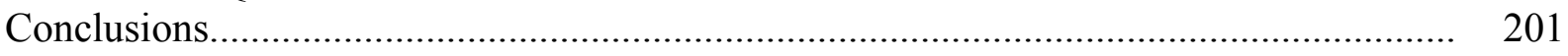

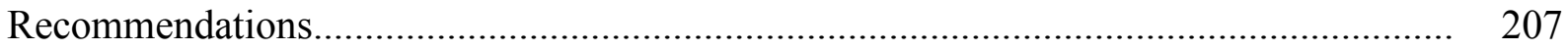

Recommendations for Practice.......................................................................... 207

Recommendations for Further Research.......................................................... 210

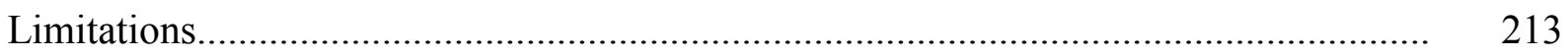

Ending Thoughts............................................................................................... 215

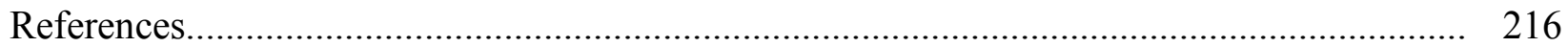

Appendix A: Program of Study ................................................................................... 241

Appendix B: NURS 216 HPS Scenarios 2006/2007......................................................... 243

Appendix C: NURS 216 HPS Scenarios 2007/2008........................................................ 246

Appendix D: Orientation to Simulation Materials.............................................................. 248

Appendix E: Simulation Prep Worksheets................................................................. 252 
Appendix F: Simulation Curriculum with Objectives....................................................... 257

Appendix G: Institutional Review Board Exempt Letter.................................................... 262

Appendix H: West Virginia University at Parkersburg Approval......................................... 264

Appendix I: Elsevier/HESI Nurse Researcher Agreement................................................. 266

\section{List of Figures}

Figure 1 The Nursing Education Simulation Framework................................................... 22

Figure 2 Trend Analysis of HESI A2 and CT Admission Exam Means Across Cohorts....... 140

Figure 3 Trend Analysis of HESI E ${ }^{2}$ Mean Scores Across Cohorts...................................... 176

Figure 4 Trend Analysis of HESI E ${ }^{2}$ CT Means Across Cohorts......................................... 181

\section{List of Tables}

Table 1 Demographic Data of Sample by Cohort............................................................. 138

Table 2 Descriptive Statistics for Demographics by Cohort............................................... 138

Table 3 Descriptive Statistics for Admission Criteria and Critical Thinking by Cohort....... 139

Table 4 ANOVA Results for Admission Criteria and Critical Thinking by Cohort............. 139

Table 5 Pairwise Comparisons of HESI A ${ }^{2}$ and CT Exam on Admission............................ 142

Table 6 Variations in Fidelity, Exposure, Delivery, and Placement of HPS by Cohort........ 143

Table 7 Fidelity and Number of HPS and Hours Replaced for Clinical per Cohort............. 153

Table 8 HESI E ${ }^{2}$ and HESI E ${ }^{2}$ CT Test of Normality by Cohorts...................................... 174

Table 9 Test of Homogeneity of Variances Admission Criteria, HESI E ${ }^{2}$, and CT............. 175

Table 10 Descriptive Statistics for HESI E ${ }^{2}$ Scores by Cohort.............................................. 175

Table 11 Pairwise Comparisons of HESI E $E^{2}$.................................................................... 177

Table 12 Descriptive Statistics for HESI E ${ }^{2}$ CT Scores and Covariate by Cohort................. 179

Table 13 Pairwise Comparisons of HESI E ${ }^{2}$ CT................................................................. 182 


\section{Chapter One: Introduction to the Study}

Nurses are essential to providing high quality health care and their education and training directly affect the safety and quality of patient care (Institute of Medicine [IOM], 1999, 2004, 2010; Smith \& Crawford, 2003). Research supports the importance of nurses and their impact on patient care (Clarke \& Aiken, 2003; Long, 2004). An adequate number of experienced nurses is essential for providing safe patient care. Reductions in the nursing workforce, often used in efforts to increase efficiency in hospitals or as a result of the nursing shortage, increase the nurse"s patient care load. Increased patient care loads can result in inadequate nursing care, which has been directly related to increases in patient mortality and morbidity (Long, 2004). The effectiveness of nursinge's $24 / 7$ surveillance to detect early signs of possible complications of patients in an acute care setting is dependent on low patient-nurse ratios and a balance of experienced and novice nurses (Clarke \& Aiken, 2003). Failure-to-rescue (inability to save a hospitalized patient"s life when complications occur) rates are lower in hospitals that are staffed with an adequate number of registered nurses (RNs). As noted in the Institute of Medicine (IOM, 2004) report addressing the work environment of nurses, "How well we are cared for by nurses affects our health, and sometimes can be a matter of life and death" (p. 2).

Educational programs that prepare nurses to sit for the National Council Licensure Exam for Registered Professional Nurses (NCLEX-RN) are faced with the challenge of not only preparing more nurses for entry into practice, but also ensuring that their graduates are prepared with the skills and knowledge to meet the increasing demands of the profession. Due to advances in technology, increased complexity and acuity of patients with shorter lengths of hospital stay, tremendous expansion of knowledge in nursing and medicine, as well as the public"s expectation to receive safe, quality health care, the complexity of health care systems in which nurses must practice is increasing (Benner, Sutphen, Leonard, \& Day, 2010; Jeffries, 
2007; Long, 2004). A greater degree of skill and knowledge, coupled with an increased ability to think critically for clinical problem solving and decision-making, is required for nurses to provide safe, effective care within the complexity of todayes health care environments (Del Bueno, 2005; IOM, 2001; Jeffries, 2007; Lisko \& O'Dell, 2010; Smith \& Crawford, 2003). Educational programs preparing nurses for practice are being challenged to ensure that graduates have the necessary knowledge, skill, and higher order critical thinking skills required to provide safe, quality, cost effective care (Durham \& Alden, 2008; National Council of State Boards of Nursing [NCSBN], 2005; National League for Nursing [NLN], 2003). To ensure that nurses are able to meet this challenge, the education system needs to adapt (IOM, 2011). The need for a transformation in nursing education, including innovative approaches to better prepare nurses for today ${ }^{e e}$ s healthcare system, is well supported by researchers, professional organizations, governmental groups, and health care organizations (American Association of Colleges of Nursing [AACN], 2008; Benner et. al., 2010; IOM, 2003, 2011; Jeffries, 2005; Long, 2004; NCSBN, 2005, 2009a; NLN, 2003). Use of human patient simulation (HPS) in nursing education programs is being seen as an effective innovative approach to better prepare students for the complexities of clinical practice (Beyea \& Kobokovich, 2004; IOM, 1999; Jeffries, 2007; Nehring, 2008; Spunt, Foster, \& Adams, 2004).

\section{Support for HPS}

In addition to the challenges associated with improving the quality of education, educational programs that prepare registered nurses are being pressured to expand enrollment to address the publicized nursing shortage. Although total enrollment in all nursing programs leading to the baccalaureate degree has increased in the last ten years, 54,686 qualified applicants were turned away from entry level BSN programs in 2010 (AACN, 2011). These findings are 
further supported by the National League for Nursing ${ }^{e e}$ (NLN, 2011) findings from their annual surveys of schools of nursing. Pre-licensure programs, responding to the survey, that prepare registered nurses report that $42 \%$ of all qualified applicants were rejected in 2010 . Of these, the highest number of rejections occurs in associate degree nursing programs (ADN), with these programs rejecting $46 \%$ of qualified applications as opposed to BSN programs turning away $37 \%$ and diploma programs 21\%. In West Virginia (WV), despite increases in admissions of most nursing schools, ADN programs turned away 642 of the 1,471 qualified applicants and BSN programs turned away 422 of the 983 qualified applicants in 2007 (Napier, 2007).

Nursing educational programs are faced with barriers that prohibit them from increasing admissions to accommodate the high number of qualified applicants interested in pursuing a career in nursing. The primary barriers cited that prohibit program expansion and increased student admissions included a shortage of qualified nurse educators and clinical preceptors, insufficient classroom space, and insufficient clinical placement sites (AACN, 2008; NLN, 2011). Although the shortage of qualified nurse educators has been directly related to the nursing shortage and cited as a major barrier for increasing enrollments for nursing schools, lack of sufficient and quality clinical placement sites is increasingly becoming more of an issue and has been cited as the primary obstacle to expanding admissions in pre-licensure nursing programs (NLN, 2011). Nursing educational programs frequently cite lack of clinical placements for courses in obstetrics, pediatrics, and mental health (Hayden, 2010). As a result, educational programs that prepare nurses have had to develop alternative learning strategies in lieu of, or to supplement, clinical experiences. With advances in computer technology, the field of HPS has provided nursing faculty with a promising complement to the traditional direct patient care clinical experiences (Durham \& Alden, 2008; Hovancsek, 2007; Hyland \& Hawkins, 
2009; Larew, Lessans, Spunt, Foster, \& Covington, 2006; Jeffries, 2008; Li, 2008; Pacsi, 2008;

Scherer, Bruce, Graves, \& Erdley, 2003; Seropian, Brown, Gavilanes, \& Driggers, 2004; Tanner, 2006a).

The use of simulation in nursing programs has dramatically increased in recent years (Jeffries, 2008). Simulation has been defined as "activities that mimic the reality of a clinical environment and are designed to demonstrate procedures, decision-making, and critical thinking through techniques such as role playing and the use of devices such as interactive videos or mannequins" (Jeffries, 2005, p. 97). Mortan (1995) defined simulation as the, intent to replicate some or nearly all of the essential aspects of a clinical situation so that the situation may be more readily understood and managed when it occurs for real in clinical practice" (p. 76). As noted by Nehring and Lashley (2009) in their review of nursing simulation, in addition to the anatomical models, task trainers, and human patient simulators, other forms of simulation used in nursing education include games, role playing, computer-assisted instruction (CAI), standardized patients, and virtual reality. Advantages, as well as disadvantages of these forms of simulation have been identified by nurse educators; however, research on the efficacy of their use is limited or has revealed mixed results (Cant \& Cooper, 2010; Nehring \& Lashley, 2009).

Although many forms of simulation are being used in nursing education today, this study focused on simulation with the use of high-fidelity HPS. High-fidelity HPS involves the use of computerized full-body mannequins that can be programmed to respond physiologically to the actions of the student. These simulations are conducted in a realistic environment with the use of actual supplies and medical equipment (Cant \& Cooper, 2010; Medley \& Horne, 2005). Learning experiences with the use of high-fidelity simulators present nurse educators with the most advanced technology in simulation education (Nehring, Lashley, \& Ellis, 2002). 
Unlike experiences with direct care patients, high-fidelity HPS provides nursing students with the opportunity to learn in a safe setting without the anxiety associated with the fear of harming an actual patient (Spunt et al., 2004). Advantages of HPS, reported in the literature, include the success of this teaching strategy on improving students "enfidence level and enhancing student learning by maintaining clinical skills, acquiring new skills, and managing crises (Haskvitz \& Koop, 2004). Student and faculty perceptions of satisfaction and value of HPS have been found to be positive in a number of studies (Bearnson \& Wiker, 2005; Bremner, Aduddell, Bennett, \& VanGeest, 2006; Feingold, Calaluce, \& Kallen, 2004; Kuznar, 2007). Students perceived that experience with HPS emulated real-life situations, enhanced learning, and increased clinical confidence (Bremner et al., 2006; Kuznar, 2007). A study examining the use of HPS in nursing programs reported that reasons for using this technology related to comfort and confidence, synthesis of knowledge, and promotion of critical-thinking and clinicalreasoning skills as reasons for using this technology (Nehring \& Lashley, 2004a).

\section{Statement of the Problem}

Although high-fidelity HPS is becoming more widely used in nursing programs, there continues to be a need for further research that provides evidence of knowledge and clinical competencies gained when HPS is used as a complement to or in lieu of traditional clinical experiences (Durham \& Alden, 2008; Gaba, 2004; Harder, 2010; Hayden, 2010; Jeffries, 2009; Li, 2008; NCSBN, 2010; Nehring \& Lashley, 2009). Substitution of traditional clinical experiences involving direct patient care with simulation continues to raise questions that need to be explored (NCSBN, 2009a). What is the most appropriate mix of hands on clinical experiences and simulation to achieve the best outcomes (Jacobson \& Grindel, 2006)? As Jeffries (2009) noted in an editorial in Nursing Education Perspectives, further research is 
needed to determine what clinical experiences with real patients provide that cannot be provided in simulation experiences. The need for further research to determine if simulation is as effective as traditional clinical hours to achieve the outcomes of critical thinking and nursing knowledge was the underlying force for this study. This study examined the relationship of critical thinking skills, knowledge, and use of HPS in the education of nurses in a pre-licensure associate degree nursing program. The impact on students ${ }^{\text {ee }}$ critical thinking skills and nursing knowledge, when HPS was used as an adjunct to clinical experiences, was explored. Differences in critical thinking skills and nursing knowledge were compared based on the fidelity of HPS used, as well as the amount of time in which the student participated in HPS.

\section{Purpose and Research Questions}

The purpose of this study was to examine relationships among fidelity and dose (amount) of HPS, critical thinking skills, and knowledge in a sample of undergraduate students in an associate degree nursing program in one selected institution in West Virginia. The overarching research question to be answered was: How does the use of HPS impact critical thinking skills and knowledge of graduates of an associate degree nursing program?

Specific questions designed to answer this research question and to identify any differences in critical thinking skills and knowledge acquisition among cohorts exposed to different types of fidelity and number of hours of HPS were:

1. How does nursing knowledge, as determined by the HESI $E^{2}$ score, of graduating associate degree nursing students trained with high-fidelity HPS compare to those trained with medium-fidelity HPS or those not exposed to HPS? 
2. Is there a difference in knowledge, as determined by the HESI $E^{2}$ score, among the cohorts of graduating associate degree nursing students based on the number of hours traditional clinical experiences were substituted with HPS?

3. How do the critical thinking skills, as determined by the Health Education Systems, Inc. Exit exam (HESI E ${ }^{2}$ ) Critical Thinking (CT) score, of graduating associate degree nursing students trained with high-fidelity HPS compare to those trained with mediumfidelity HPS or those not exposed to HPS?

4. Is there a difference in critical thinking skills, as determined by the HESI E ${ }^{2}$ CT score, among the cohorts of graduating associate degree nursing students based on the number of hours traditional clinical experiences were substituted with HPS?

\section{Background}

Critical thinking in nursing education. Prior to 1960, the majority of nurses in the U.S. were trained in hospital-based diploma programs and the majority of their training consisted of working on the floor administering patient care (Benner et al., 2010; Cronenwett, 2011). The transition of nursing education programs into institutions of higher education occurred in the 1940s, with the first associate degree program developed in 1958 (Benner et al., 2010). As noted by Benner, Sutphen, Leonard, and Day (2010), the move from service-driven hospital-based diploma programs to more structured and formalized BSN and ADN programs offered through institutions of higher education has created a separation of academia and practice. Basic components of the curriculum in today ${ }^{\text {ee }}$ s nursing programs consist of classroom instruction and planned laboratory experiences in skills laboratories and clinical laboratories. With this curriculum, students learn nursing knowledge (theory) in a classroom setting, practice skills 
using equipment and technology seen in the clinical setting in a skills laboratory, and apply the knowledge and skills in the clinical setting when caring for patients (Benner et al. 2010).

Although there are three pathways for entry into the profession of nursing: diploma, $\mathrm{ADN}$, and $\mathrm{BSN}$ programs, the purpose of each is to prepare graduates with the necessary knowledge and skills to attain licensure through successfully passing the NCLEX-RN (Benner et al., 2010). The NCLEX-RN is a computerized multiple-choice exam designed to demonstrate minimal competency by requiring the graduate to think at the application, analysis, and synthesis levels of cognitive ability (Del Bueno, 2005).

Nurse educators are challenged to teach students to think critically and are responsible for creating opportunities for students to develop critical thinking and critical reasoning skills (NLN, 2005a). The NLN (2000) has identified clinical decision-making as a core competency of graduates of associate degree nursing programs. Evidence based practice and the use of critical thinking have been identified by the NLN as providing the foundation for appropriate clinical decision-making. As a result, critical thinking can be seen as a common thread throughout the curricula of most nursing educational programs (AACN, 2008; Daly, 1998; Hicks, 2001; Jones \& Brown, 1991; Patterson, Crooks, \& Lunyk-Child, 2002; Paul, 1995; Simpson \& Courtney, 2002; Su \& Juestel, 2010).

Findings of a multiyear study of nursing education, funded by the Carnegie Commission, were published in the book Educating Nurses: A Call for Radical Transformation by Benner et al. (2010). Results of the study identified that most nurse educators rely heavily on lecture, supported by audiovisuals, in their presentation of classroom knowledge. The passive learning that occurs as a result of lectures promotes rote memorization of facts and principles. The authors noted that lectures are overloaded with a vast amount of information and content that 
instructors believe needs to be covered; include large amounts of information that is required to be memorized; and lack pedagogical strategies that actively involve students, facilitate critical thinking, and provide for integration of knowledge acquisition and use. As a result, students reported feeling overloaded, experienced a decreased retention of subject matter, and found it difficult to transfer knowledge from the classroom to clinical setting when administering patient care. Although the authors identified that clinical situations and information to guide clinical decisions can be presented through lectures, students are passive recipients of the critical thinking required in the reasoning process for these situations (Benner et al., 2010). The literature supports that active learning increases the retention of knowledge (Johnson, Zerwic, \&Theis, 1999) and enhances critical thinking skills (Billings \& Halstead, 2009). More active forms of learning must be integrated into nursing curricula that provide critical thinking experiences (Lisko \& O'Dell, 2010) and build on a student"s knowledge and skills (Sinclair \& Ferguson, 2009).

Experiential learning through clinical experiences. Clinical experiences, where students begin working with patients in clinical settings, are usually started early in a nursing program and provide the opportunity for students to learn through experience (Benner et al., 2010). As defined by Benner et al. (2010), experiential learning is learning that occurs from the experience of caring for patients; the learning from the particular situations of specific patients is referred to as situated learning. Benner et al. also noted that students learn best through the experiential and situated learning that occurs as a result of working with actual patients in clinical settings. Supported by Kolb"s (1984) experiential learning theory, clinical situations with patients provide the learner with an expanded knowledge base. Each experience the students encounter, through reflection, conceptualization, and incorporation of the meaning of 
the experience into their existing knowledge base, adds to the knowledge base that can be applied to new situations (Waldner \& Olson, 2007). Situated learning that occurs through clinical experiences provides the opportunity for students to live the subject matter of nursing in the context of real-world environments (Stein, 1998). As Stein (1998) noted, the opportunity to experience the complexity and ambiguity of learning in the real world increases knowledge and transfers learning from the classroom to practice.

The inclusion of planned, structured, supervised clinical experiences across the life-span in pre-licensure nursing educational programs is essential to the education of nurses (AACN, 2008; Benner et al., 2010; NCSBN, 2005). The National Council of State Boards of Nursing [NCSBN] (2005) conducted a study of clinical education to ensure that new graduates are prepared to practice safely and begin discussions of alternative activities that could be used in lieu of traditional clinical experiences in pre-licensure nursing educational programs. The NCSBN's study, which included a review of the literature, a survey of boards of nursing and nursing education organizations, and input from stakeholders and experts, confirmed the need for situated learning and practice in the authentic situation. In particular, the need for feedback and reflection was identified as being important for developing knowledge and critical thinking, as well as improving confidence of students. As a result of this study, it was the recommendation of the NCSBN (2005), in their position paper on Clinical Instruction in Pre-licensure Nursing Programs, that pre-licensure nursing education programs include clinical experiences with actual patients. This recommendation was further supported by a position statement, issued in 2004 by the American Organization of Nurse Executives (AONE), recommending the inclusion of structured, supervised, clinical experiences in all pre-licensure programs (NLN, 2005b). In addition, the Essentials of Baccalaureate Education for Professional Nursing Practice (AACN, 
2008) clearly states "patient care experiences with actual patients form the most important component of clinical education" (p. 34). However, the value of HPS experiences in augmenting clinical learning is recognized in the Essentials of Baccalaureate Education for Professional Nursing Practice.

Transitioning from academia to practice. A sharp divide between the theory that is learned in the classroom and the need to know how to apply the theory in clinical experiences and practice settings has been associated with negative consequences and has created challenges for nursing graduates, nurse educators, and health care settings (Benner et al., 2010). Recent studies support the lack the preparation of new graduates to practice effectively in health care settings (Del Bueno, 2005; IOM, 2011; Kovner, Brewer, Yingrengreung, \& Fairchild, 2010; Pellico, Brewer, \& Kovner, 2009; Smith \& Crawford, 2003). Results from the 2002 NCSBNS practice and professional issues survey of newly licensed RN"s (Smith \& Crawford, 2003) found that $49 \%$ of entry level nurses reported they had been involved in errors. Gaps in educational preparation for practice were identified by entry level nurses as a contributing factor to these errors. A follow-up report in the fall of 2004 (Kenwood \& Zhong, 2006) found that 53\% of entry level RN's reported they were involved in errors. Although the majority of nurses who were surveyed reported satisfaction with their educational preparation, new nurses perceived their involvement in errors were related to the adequacy of their educational preparation. Del Bueno (2005) reported that the majority of new graduates of nursing programs have difficulty translating knowledge into practice and as few as 35 percent of new graduates meet entry level expectations for clinical judgment.

To overcome the gap from academia to practice, classroom learning must be better integrated with clinical experiences (Benner et al., 2010). Integration provides opportunities for 
students to make the connection between acquiring knowledge and using knowledge to develop clinical reasoning skills required for practice (Benner et al., 2010; Tanner, 2008). As stated in the Essentials of Baccalaureate Education for Professional Nursing Practice (AACN, 2008), learning opportunities must be sufficient to ensure graduates are prepared to use clinical/critical reasoning to achieve the outcomes and integrate the knowledge and skills required for professional nursing practice. As noted earlier, critical thinking skills are essential for nurses to make clinical judgments and decisions in the complexity of health care systems. Instructional strategies used in both didactic and clinical components of nursing courses strive to influence the development of psychomotor skill performance while enhancing critical thinking and clinical decision-making ability (Durham \& Alden, 2008). The increased use of high-fidelity HPS in nursing education programs over the last few years supports the observation that nurse educators are beginning to recognize the value of supplementing didactic and clinical components with simulation to enhance critical thinking and decision-making (Jeffries, 2008).

It is important to note that traditional clinical experiences with patients do have limitations (Benner et al., 2010). The types of experiences that students are exposed to are dependent upon the clinical placement of the student and the diversity of patients (Jeffries, 2007; Nehring, 2010). Faculty cannot guarantee consistency among student experiences or that all students will be provided with the opportunity to care for a variety of patients. Furthermore, when critical situations arise during clinical experiences that require quick decisions and actions, the nurse in charge takes over to ensure the safety of the patient (Durham \& Alden, 2008). Experiences that could have provided excellent opportunities for students to use their critical thinking skills for clinical decision-making are often lost. Barriers to student experiences are also imposed by practice boundaries or agency limitations (Li \& Kenwood, 2006). As Li and 
Kenwood (2006) noted, opportunities for student nurses to receive and write verbal orders from a physician or implement certain skills may be lost due to practice boundaries or agency restrictions.

Supplementing student clinical experiences with HPS can overcome these limitations imposed by traditional clinical experiences. Standardization of student experiences occurs when all students participate in a patient scenario designed to meet the objectives of the clinical experience (Ironside \& McNelis, 2010; Nehring, 2010, Pacsi, 2008). Scenarios designed to expose students to high stakes critical incidents provide learning opportunities for the student to gain experience (Beyea \& Kobokovich, 2004; Nehring et al., 2002). As Beyea \& Kobokovich (2004) indicated, these situations require the student to utilize critical thinking for clinical decision-making and problem solving in a safe environment without the risk to patient safety. Patient outcomes of HPS can either be positive or negative based on the decision-making and actions of the students. Debriefing that occurs after the simulation experience provides students with the opportunity to reflect on their performance during the simulation experience and to suggest alternative solutions (Jeffries \& Rogers, 2007; Johnson-Russell \& Bailey, 2010).

The argument for the use of HPS in nursing educational programs. In addition to the challenges facing nurse educators to close the gap between nursing education and practice, nurse educators and students must be in tune to the dangers associated with the high-stakes learning that occurs when students are placed in an acute care hospital for clinical experiences (Benner et al., 2010). The high acuity of patients, often with complex needs and disease processes, requires nursing students to apply the knowledge, skills, and attitudes necessary to ensure the safety of their patients (Del Bueno, 2003; IOM, 2001; Jeffries, 2007). As Benner et al. (2010) noted, the threat of making a mistake that could potentially result in injury or death of a patient is very real. 
It has been estimated that at least 44,000 or more people die each year in hospitals as a result of preventable medical errors (IOM, 1999). In efforts to improve the safety and quality of healthcare, the Institute of Medicine (IOM) issued their first report on a comprehensive strategy for prevention of medical errors, To Err is Human: Building a Safer Health Care System. Released in November 1999, a minimum goal to reduce medical errors by $50 \%$ over the next five years was identified in the report. Using simulation whenever possible was one recommendation to prevent errors in the clinical setting. As stated in the IOM report (1999), “...teaching institutions should participate in the development and use of simulation for training novice practitioners, problem solving, and crisis management, especially when new and potentially hazardous procedures and equipment are introduced" (p. 179). The recommendation for the use of simulation in nursing educational programs is further supported by the NCSBN"s (2005) position paper on clinical education. As stated in the position paper (NCSBN, 2005), it is the recommendation that clinical experiences in pre-licensure nursing programs include actual patients; however, these clinical experiences might also include "innovative teaching strategies that complement clinical experiences for entry into practice competency" (p. 1). Although it was noted that simulation cannot replace learning in the authentic setting, simulation was identified as an important asset to clinical learning.

The use of simulation in the education of nurses is not new and can be traced back to the nineteenth century (Cooper \& Taqueti, 2004). Cooper and Taqueti (2004), in an article on the history of the development of mannequin simulators used for healthcare training, defined simulation as "applications of simulators for education or training" (p.i11). Simulators, defined as "physical objects or representation of the full or part task to be replicated" (p. i11), are designed to recreate the environment to imitate tasks (2004). The degree to which the simulator 
mimics reality is referred to as fidelity (Nehring \& Lashley, 2009) and is divided into three categories: low-, medium-, and high-fidelity (Seropian et. al., 2004). As noted by Seropian et al. (2004), low-fidelity simulators are static and lack the detail and realism of medium- to highfidelity simulators. High-fidelity simulators are the most realistic mannequins with the physiological detail that brings students closer to believing that what they are seeing and experiencing is real.

A national survey of 1,060 pre-licensure nursing programs, conducted by the NCSBN in 2010 , indicated that $87 \%$ of students receive high- or medium-fidelity experiences (Hayden, 2010). Simulation was reported to be used in five or more courses in $55 \%$ of the programs responding to the survey. Additionally, respondents in the survey listed, among others, clinical decision-making/critical thinking as one type of learning opportunity offered by their simulation laboratory (2010). Li (2008), in a presentation at the NCSBN conference on March 26, 2008, cited the opportunity for students to engage in critical thinking, decision-making, and delegation as one potential advantage of simulation over actual clinical experience. The value of HPS as a means to improve student confidence, decision-making, and critical thinking was identified through a review of the literature conducted by Nehring, Ellis, and Lashley (2001).

Advantages of incorporating HPS in pre-licensure nursing educational programs are well cited in the literature and will be discussed in more detail in chapter two. The opportunity for students to be active participants in their learning without risk to an actual patient has been cited as the major advantage of using HPS (Gaba, 1992). In the era of healthcare reform and the publices expectation that nurses and other health care professionals demonstrate professional competency, nursing educational programs have the responsibility to ensure that competency of their nursing graduates is not achieved at the expense of patient safety (Decker, 2007c; 
McCallum, 2007). HPS affords nursing educational programs the opportunity to enhance competency of nursing students and promote safe nursing care in a controlled learning environment (Durham \& Alden, 2008). Exposing students to HPS provides a bridge between theory and clinical practice (Pasci, 2008; Rauen, 2001). Scenarios can be selected based on the learning needs of the student for accomplishment of course objectives. Unlike experiences with actual patients, learning that occurs with HPS provides a safe and realistic environment to help students understand and apply the cognitive and psychomotor skills learned in the classroom, without fear of harming an actual person (Alinier, Hunt, \& Gordon, 2004; Spunt et al., 2004). Students are expected to apply previously learned theory and draw from previous clinical experiences which provide opportunity for students to actively participate and practice critical thinking, clinical decision-making and psychomotor skills required in actual clinical practice (Durham \& Alden, 2008; Sinclair \& Ferguson, 2009).

Use of HPS as an alternative or supplement to traditional clinical experiences is gaining attention. With the capability of nursing faculty to control the types of case scenarios used with high-fidelity simulators, students can be provided with situations they may not encounter during clinical experiences with direct patient care (Pacsi, 2008). As noted by Pacsi (2008), HPS can control for inconsistencies in patient assignments that can occur with student"s clinical experiences; students are exposed to the same scenarios under the same conditions, providing greater consistency in learning experiences. In addition, HPS provides opportunity for students to be exposed to emergent critical situations that they typically are not able to participate in during traditional clinical experiences (Gaba, 2004). To ensure the safety of patients, the staff typically takes over patient care when a patientes condition deteriorates and the student assumes the role of passive observer (Durham \& Alden, 2008). Therefore, HPS has gained popularity as 
an adjunct to direct patient care in traditional clinical experiences when quality clinical sites are unavailable.

Although the advantages of HPS are well cited in the literature, it is important to recognize the challenges associated with the use of simulation. One of the most frequently cited limitations to HPS is the cost. Initial costs of the purchase of human patient simulators vary depending on the fidelity and can range from $\$ 10,000$ to $\$ 200,000$ (Lapkin \& Levett-Jones, 2011). Additional costs identified by Lapkin and Levett-Jones (2011) involve remodeling or creation of physical space and purchase of equipment to replicate a hospital environment for the simulator, a remote area for debriefing, a storage space for equipment, and a control area for faculty to run the simulation. Video recording equipment for debriefing, the purchase of consumables and supplies to be used by students during simulations, purchase of scenarios or release time for faculty to develop, and training for faculty and staff to use competently the technology are additional costs (Hovancsek, 2007; Lapkin \& Levett-Jones, 2011; Rauen, 2001). Further research is needed to evaluate if the outcomes of HPS outweigh the costs (Hovancsek, 2007; Norman, 2003).

\section{Significance of Study}

National significance. Although the number of publications related to simulation has increased in the past few years, student outcomes as a result of simulation is an area that has been identified as needing more research (Cant \& Cooper, 2010). It is important to validate whether HPS makes a difference in helping students learn how to provide safe, competent care when compared to more traditional pedagogies. This study specifically explored the relationship of fidelity and amount of exposure to HPS to critical thinking and knowledge of graduates of an associate degree nursing program. Statistical analysis compared critical thinking abilities and 
knowledge of students exposed to low-fidelity HPS, medium-fidelity HPS, and high-fidelity HPS. In addition, critical thinking abilities and knowledge were compared among cohorts who had no exposure to HPS and cohorts who had differing numbers of traditional clinical hours substituted with HPS.

The role of HPS as a teaching strategy is being questioned by many nurse educators and the NCSBN. Individual state Boards of Nursing (BONs) are investigating how HPS is being incorporated into the curricula of schools of nursing (NCSBN, 2009b). Most BONs are waiting for more research to determine what role simulation should have in nursing education in relation to clinical education ( $\mathrm{Li}, 2008)$. The NCSBN (2009b) surveyed BONs regarding their position on simulation and clinical experiences. Of the 40 boards that responded, seven (18\%) stated their education rules addressed simulation. Rules varied ranging from not permitting simulation to be used in lieu of direct patient care hours to specific guidelines of the percentage of time that direct patient care can be substituted with simulation. Seven BONs reported that they specify the percentage of time that clinical, with direct patient care, can be replaced with simulation. One BON permitted "no more than 100 clock hours of the required 800 hours of clinical practice" (pg. 4), one BON allowed for "20\% of direct client care hours being simulation experiences" (pg. 2), and two allowed for "no more than 25\%" (p.4) of clinical hours to be used for simulation or hours other than direct patient care. Three BONs did not permit simulation to take the place of clinical experience with actual patients (p. 4). Some BONs provided flexibility to the nursing educational program. The education rules of these BONs stated that simulation can be "used as a complement to clinical learning experiences" (p.2), "clinical experiences might also include simulated activities" (p.4), and "utilization of simulation experiences is at the discretion of the nursing program" (p.4). 
As noted in the NCSBN survey (2009b), the BONs have very diverse rules related to the percentage of time that can be allocated for simulation because of the relative lack of research published. Based on comments from the survey, BONs are beginning to collect data regarding the amount of simulation being used in the schools of nursing and have plans to revise their rules to include language for simulation after data collection is complete. As one Board stated, "We are asking our schools to do no more than $25 \%$ of active simulation for clinical. We do not feel that research is sufficient - to determine a number of hours to be put in the rules" (p.5).

Comments from the survey did address the need for further research on simulation, particularly related to student outcomes. This research study adds to the body of knowledge regarding the impact of HPS on student outcomes of critical thinking and knowledge gains.

Local significance. Although the WV Board of Examiners for Registered Professional Nurses (WVBERN) does not have a position statement regarding simulation being used in lieu of traditional clinical experiences, it does support the use of simulation in nursing education (L. Rhodes, personal communication, February 2, 2012). Permission to substitute simulation for a small percentage of direct patient care has been granted to schools. Initial research on simulation conducted by the NCSBN provided inconclusive results $(\mathrm{Li}, 2008)$. Although further research on simulation is in progress, the WVBERN has no current plans to change the rules regarding clinical or simulation (L. Rhodes, personal communication, February 2, 2012).

The institution in this study has spent an enormous amount of money for the purchase of two adult, a pediatric, and a baby high-fidelity HPS. In addition, the campus lab was enlarged and remodeled to include three glass enclosed rooms that replicate a hospital room. A full time lab manager was hired to assist with set ups and running the simulation and the teaching load of one faculty member has been devoted $100 \%$ to simulation. Just recently, two additional adult 
simulators were purchased. HPS has replaced one hour of traditional clinical hours each week across the program of study and research was needed to determine the impact that this has had on student outcomes. The National League for Nursing Accrediting Commission, Inc. [NLNAC] (2008) requires programs to evaluate student learning to determine if program outcomes are met. Improvement in critical thinking skills and success on the NCLEX-RN are two program outcomes of this institution. This study has been helpful in determining what effect the integration of HPS has had on the graduate s critical thinking skills and knowledge for successful passage of the NCLEX-RN. Results of the study will be used to determine if additional direct patient care clinical hours will be substituted with HPS. Additionally, results of this study can provide guidance to other nursing programs and their regulatory bodies as they strive to balance the most appropriate ratio of direct patient care hours and simulation hours.

\section{Limitations of Study}

This study is an ex post facto study in that it uses data over a span of six years from cohorts of students who have already graduated from a nursing program. Critical thinking and knowledge scores, obtained from a comprehensive exit exam, were compared among cohorts of graduates exposed to different fidelity and amounts of HPS during their educational program. Using the Statistical Package for the Social Sciences (SPSS), an analysis of variance (ANOVA) was performed to determine if there were differences in critical thinking and knowledge in relation to the type (low-, medium-, or high-fidelity) and amount of student exposure to HPS.

It is important to note that this study is not a direct cause-effect study. With the study spanning the years of 2004 through 2010, it was not possible to control for the many variables that might also have had an impact on the critical thinking and knowledge of graduates of the program, in addition to the implementation of HPS. The inability to control for all variables that 
could have affected the critical thinking and knowledge scores of graduates is a significant limitation of the study. To control for the variables of differences found among nursing curricula such as resources, support for students, assessment outcomes, and type of degree obtained among institutions offering a nursing education program, this study was confined to graduates from one institution, a community college offering the associate degree in nursing. Although the curriculum of this nursing program has remained the same, there has been high faculty turnover, in both the classroom and clinical laboratories, throughout this time frame. Differences in instructor experience, teaching style, strategies used for classroom instruction, program policies, and expectations required of the students may have had an effect on the development of a student"s critical thinking skills and acquisition of knowledge.

Implementation of a required remediation program was incorporated in fall 2008. This program required all students who did not earn a $76 \%$ on any unit exam to attend remediation. The remediation coordinator reviewed the content missed on the exam with the student. Although not all students attended remediation, the implementation of this program is cited as a limitation of the study. Exposure to remediation may have had an influence on the knowledge and critical thinking of those students who participated in simulation.

With the study confined to associate degree nursing education and one institution, there is a potential limitation to generalizability of the study"s findings. Although nursing students from an associate degree program take the same NCLEX - RN as graduates from a diploma and baccalaureate program, differences in prerequisites and admission criteria of programs, organization and content of curricula, and learning styles of traditional versus non-traditional students may have an impact on the effectiveness of simulation. 


\section{Conceptual Framework}

The theoretical framework that guided this study is Jeffries"e theoretical framework for simulation design (Jeffries, 2005; Jeffries, 2007). The model provides the details to assist educators with the design, implementation, and evaluation of simulation and is depicted in Figure 1. As portrayed in the model, the relationship of five major components, each comprised of specific variables, influences the success of simulation. The five major components include: teacher factors, student factors, educational practices that need to be incorporated into the simulation, design characteristics of the simulation, and expected student outcomes.

Figure 1. The Nursing Education Simulation Framework

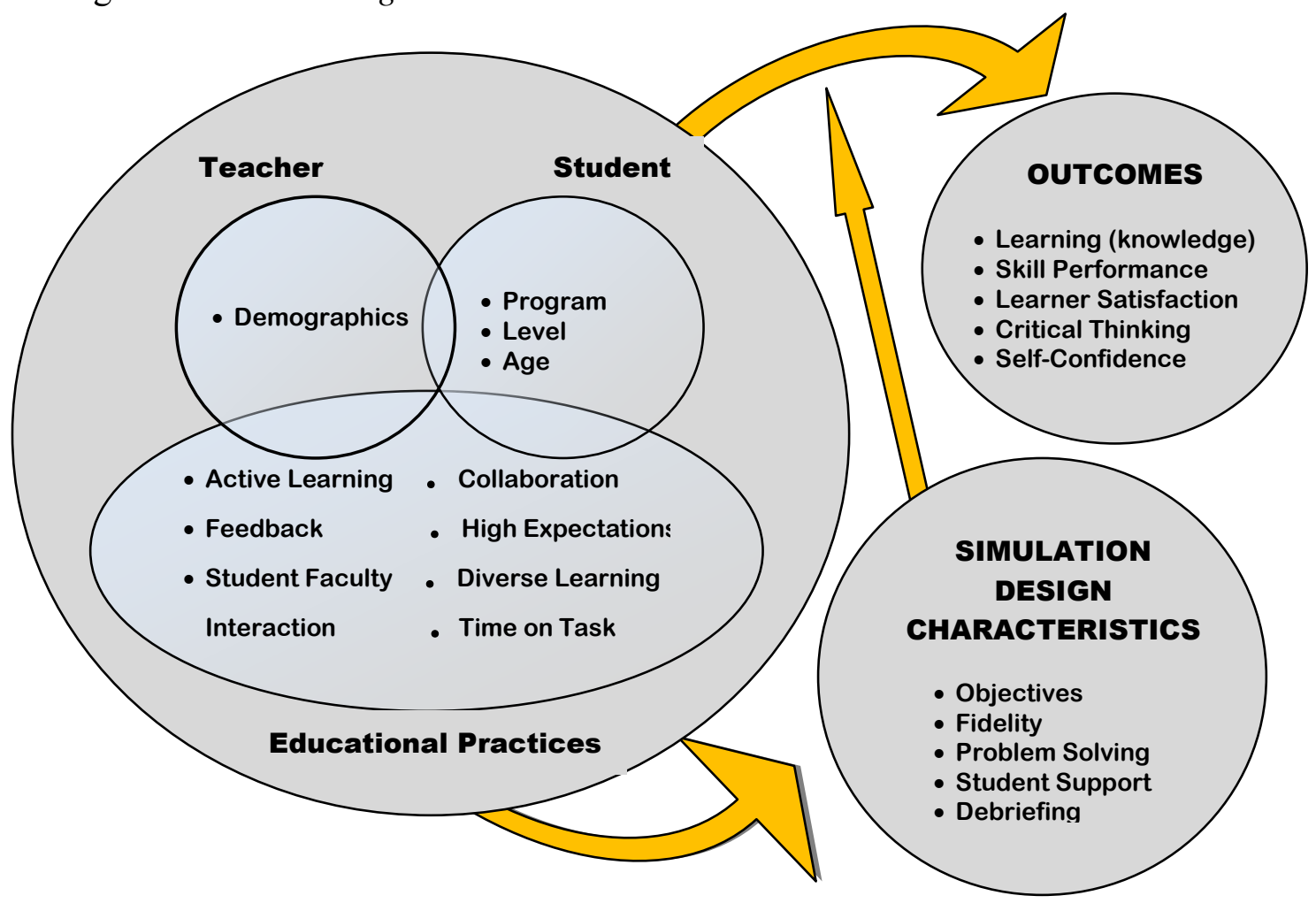

From Simulation in Nursing Education: From Conceptualization to Evaluation (p. 23), by P. R. Jeffries (Ed.), 2007, New York: NLN. Copyright 2007 by the National League for Nursing. Reprinted with permission. 
As illustrated in the model, the interrelationships of the teacher, student, and educational practices are significant factors of simulation that influence the simulation design characteristics and impact student outcomes (Jeffries \& Rogers, 2007). As acknowledged by Jeffries and Rogers (2007), the teacher is essential to the success of the simulation experience. Serving as a facilitator of student learning, the teacher provides support and guidance throughout the experience and debriefing. Using a student-centered approach to learning, students must be selfdirected and motivated as they take responsibility for their learning. They are expected to arrive prepared for the scenario, function collaboratively within a team, and be an active participant in the debriefing. Jeffries and Rogers identified the importance of students ${ }^{\text {ee }}$ roles being clearly defined in the simulation experience and discussed the differences between the response-based role and process-based role students can assume during the simulation experience. A student assigned to observe the experience assumes the response-based role, while the student who assumes the process-based role is an active participant in the simulation.

The model distinguishes seven educational practices that need to be considered when planning simulation experiences. These seven educational practices have been identified by Chickering and Gamson as influencing student satisfaction and learning (Jeffries, 2005). Supported by the works of Dewey's (1933), Kolb"s (1984), and Schön ${ }^{\text {ee }}$ (1983) reflective thinking and experiential learning theories, the seven principles include: active learning, feedback, student-faculty interaction, collaborative learning, high expectations, time on task, and diverse learning (Jeffries, 2005). As noted by Jeffries (2005), students must be actively engaged in the simulated experience with opportunities to use critical thinking skills for decision-making and problem-solving. Supported by Dewey and Kolb, active involvement in a learning experience with opportunities for problem solving and reflection is important for learning. 
Debriefing provides opportunity for students to self-reflect on their performance and receive immediate feedback from their instructor and peers, thus reinforcing learning (McLellan, 1996). Interactions of faculty and students, during and after the simulation, facilitate discussion and learning. Students feel more comfortable asking questions in a climate of mutual respect and support (Jeffries, 2005). As Weis and Guyton-Simmons (1998) noted, students learn best when faculty are available to answer questions during a learning activity. Simulation provides an opportunity for students to collaborate as they work together and share in the problem solving and decision-making for patient care. Results of simulation can be improved when both faculty and students have high expectations for the experience, diverse learning styles are accommodated, and activities are incorporated into the simulation to meet auditory, visual, tactile, and kinesthetic learning styles (Jeffries, 2005). Fountain and Alfred (2009) found that students with both a preference for solitary learning and social learning were satisfied with HPS. To keep students on task, it is important that clear objectives are identified, a time frame is identified, simulations are limited to reinforcing only a few key concepts, orientation to the technology is provided prior to the experience, and students are briefed on the scenario prior to beginning (Jeffries, 2005).

The simulation design characteristics of objectives, fidelity, problem solving, student support, and debriefing must be planned to accomplish the purpose and intended outcomes of the simulation (Jeffries \& Rogers, 2007). Clear objectives, written to match the learner"s knowledge, course content, and desired outcomes of the experience, are the first step in planning for a simulation (Jeffries \& Rogers, 2007; Hawkins, Todd, \& Manz; 2008). Students must be provided with guidelines for preparation and how the simulation will be conducted. Orientation to the capabilities of the simulator and preparing the student for the simulation is critical (Alinier 
et al., 2004). Failure to do so can influence behavior and affect the learning experience. As Alinier et al. (2004) discussed, simulation must have a high degree of fidelity (realism) and be as authentic with realistic environmental props as possible to help students suspend disbelief and act as they would in practice. The complexity of the scenario should match the learner's knowledge and be at a level that is attainable but challenging (Jeffries \& Rogers, 2007). Jeffries and Rogers (2007) recommended that support be given to the student in the form of cues provided throughout the scenario to help the student progress through the activity. However, the authors cautioned that cues should provide only enough information to allow the student to continue and should not interfere with the studentes problem solving. Debriefing, which should occur at the end of the experience, provides opportunity for reflection on the positive aspects of the experience, as well as to identify areas for improvement (Jeffries \& Rogers, 2007).

Five possible outcomes of simulation are identified in the model: increase in knowledge or understanding, increase in self-confidence of student, increase in satisfaction of the student, improvement in skill performance, and enhancement of critical thinking skills (Jeffries, 2005). Evaluation of these outcomes is essential for measuring student learning and the effectiveness of the simulation experience. This study specifically addressed the impact of simulation on the outcomes of knowledge gain and enhancement of critical thinking skills.

Critical thinking and surrogate terms. It is important to call attention to the wide use of surrogate terms associated with critical thinking such as clinical decision-making, problem solving, diagnostic reasoning, and clinical judgment that can be found in the literature (Turner, 2005). The broad use of these terms can create confusion among educators and researchers; attempts to understand the relationship between these terms have been the subject of many 
articles (Duchscher, 1999; Facione \& Facione, 1996; Ignatavicius, 2001; Kataoka-Yahiro \& Saylor, 1994; Tanner, 2006b; Turner, 2005).

In nursing, the necessity of critical thinking skills required for effective decision-making, problem solving, diagnostic reasoning, and sound clinical judgment is evident in the literature (Alfaro-LeFevre, 1995; Dushscher, 1999; Facione \& Facione, 1996). Critical thinking skills are identified as an important step in decision-making and problem solving (Simpson \& Courtney, 2002) and are necessary for nurses to think, apply, analyze, synthesize, and evaluate clinical situations to form clinical judgments (Schank, 1990). AACN (1998) defined critical thinking as "All or part of the process of questioning, analysis, synthesis, interpretation, inference, inductive and deductive reasoning, intuition, application, and creativity" (p. 36).

Critical thinking requires not only discipline-specific knowledge, experience, and attitudes, but is also a thought process that involves higher order thinking processes of diagnostic reasoning, clinical decision-making, and problem solving necessary for clinical judgment (Boland, 2009; Kataoka-Yahiro \& Saylor, 1994). The critical thinking process is reflective and facilitates the cognitive process of diagnostic reasoning required for sound nursing judgment and problem solving (Facione \& Facione, 2008; Kataoka-Yahiro \& Saylor, 1994; Paul \& Heaslip, 1995, Tanner, 2006b). Although professional organizations have identified that the higher order thinking skills of clinical reasoning, clinical decision-making, and clinical judgments are required for nurses to provide safe, quality nursing care; critical thinking skills are recognized as the foundation and underlying skill necessary for appropriate decision-making and formation of clinical judgments (AACN, 2008; NLN, 2000; 2010). Therefore, critical thinking is seen as the broad umbrella that serves as the cognitive engine that drives diagnostic reasoning, clinical decision-making, problem solving, and clinical judgment in nursing (Hicks, 2001). 
The impact of HPS on critical thinking skills of associate degree nursing graduates was a focus of this study. The tool used to measure critical thinking was the HESI $E^{2}$ exam. Although this exam is a multiple choice exam, the concept of critical thinking is incorporated into each question. In addition to students receiving a total score for the exam, critical thinking scores are provided. Although this score is identified as a measurement of critical thinking abilities, the score measures students ${ }^{\text {ee }}$ critical thinking skills required for decision-making and clinical judgment within the context of nursing practice. Therefore, the review of literature includes a discussion of critical thinking as a broad concept; the association of critical thinking, clinical reasoning, clinical decision-making, and nursing judgment; the significance of reflective thinking on the development of critical thinking and knowledge; as well as the responsibility of nurse educators for developing these skills in their graduates.

\section{Definition of Terms}

1. Associate degree nursing program: A nursing program, typically offered by community colleges, designed to be completed in two years. Graduates earn the Associate in Applied Science degree and are prepared to sit for the NLCEX-RN.

2. Critical thinking: The definition of critical thinking as defined by the institution represented in the study is "The reflective process of clinical decision-making using problem-solving skills and involving inquiry, critical analysis, and synthesis to resolve or influence a situation" (West Virginia University at Parkersburg [WVUP], 2012). For this study, critical thinking is operationally defined by the HESI E ${ }^{2} \mathrm{CT}$ score.

3. Human patient simulation: Patient care scenario in a simulated realistic environment that uses a medium-fidelity or high-fidelity simulator. 
4. High-fidelity human patient simulator: Patient care scenario in a simulated realistic environment that uses a standardized patient or a full-body patient simulator that can be programmed to respond to affective and psychomotor changes, such as breathing chest action. High-fidelity mannequins used in this study were SimMan and IStan.

5. Medium-fidelity human patient simulator: Patient care scenario that uses a full-body simulator with installed human qualities such as breath sounds without chest rise. Medium-fidelity mannequins used in this study were LaerdalesVitalSim.

6. Low-fidelity human patient simulator: use of static mannequins for the practice of psychomotor skills.

\section{Summary}

Nursing educational programs are being challenged not only to prepare more nurses for entry into the profession, but also to change the way they educate nurses to better prepare them for the complexities of the health care system. In efforts to improve the safety and quality of patient care, professional organizations and government agencies are calling for a transformation in nursing education. Current methods of educating nurses are falling short in preparing graduates for transition into professional practice. Although traditional clinical experiences with patients provide the student with the opportunity to apply knowledge learned in the classroom, schools of nursing frequently cite a lack of sufficient clinical placement sites as the primary obstacle for program expansion. In addition, limitations imposed by the traditional clinical experiences can impact the quality of the student's education. Use of HPS in the education of nurses is being seen as a promising complement to didactic education and traditional patient care clinical experiences. 
Although advantages of using HPS are well cited in the literature, there continues to be a need for more research that provides evidence of knowledge and clinical competencies gained when HPS is used as a complement to or in lieu of traditional clinical experiences. This study specifically addresses the need identified by nurse educators, nurse researchers, and governing boards of nursing for more research that provides evidence of the knowledge and clinical competencies gained when HPS is used as a complement to traditional clinical experiences. Chapter two provides an extensive review of the published literature related to critical thinking and its importance to professional nursing, as well as experiential learning and its impact on critical thinking and acquisition of knowledge. The role of simulation in the education of nurses, advantages and disadvantages of HPS, and research related to HPS is discussed. 


\section{Chapter Two: Review of the Literature}

It is imperative that educational programs preparing graduates for the practice of professional registered nursing respond to the call for a transformation in nursing education. Graduates must be prepared with the necessary knowledge, skill, and higher order thinking to provide safe, quality care in today ${ }^{\mathrm{ee}}$ complex health care system. Nurse educators have been challenged to include innovative approaches in the preparation of nurses. Benefits associated with the incorporation of human patient simulation (HPS) into nursing education programs have shown great promise This study examined the relationship of critical thinking scores, knowledge acquisition, and use of HPS in the education of nurses in a pre-licensure associate degree nursing program.

Chapter two provides an extensive review of the literature and research related to the variables under study. An overview of critical thinking, what it is, and why it is important to nursing is examined. Discussion of experiential and situated learning theories focuses on the relationship of experiential and situated learning to the development of critical thinking and knowledge acquisition. Barriers and challenges of the current model of clinical education is addressed, as well as innovations for improvement. A brief review of the history of HPS in health care education includes the evolution and current use in nursing education. Results of previously conducted research on the benefits and limitations of the use of HPS are explored, culminating with research specific to the outcomes of critical thinking and knowledge.

\section{Critical Thinking}

Critical thinking and relevance to nursing. It is well-documented that critical thinking skills are essential skills required of professional nurses to practice safely, competently, and effectively within the complexities of the health care systems of today (Daly, 2001; Facione \& 
Facione, 1996; Ironside, 2005; Kataoka-Yahiro \& Saylor, 1994; Paul \& Heaslip, 1995; Simpson \& Courtney, 2002). It is the expectation that nursing programs prepare graduates with the higher level of thinking required for effective decision-making, problem solving, and nursing judgment essential for entry into the profession of nursing (NLN, 2010; NLNAC, 2008). The National League for Nursing Accrediting Commission Standards and Criteria for Associate Degree Nursing Programs [NLNAC] (2008) requires the curriculum to prepare students for safe practice in contemporary health care environments (Standard 4) and the graduates to demonstrate achievement of outcomes appropriate to role preparation (Standard 6.4).

The NLN (2000) identified clinical decision-making as one of the core components and competencies for graduates of associate degree nursing programs. The NLN further defined clinical decision-making as the use of critical thinking to analyze and integrate information and knowledge for clinical judgments. As emphasized by NLN, effective clinical decision-making provides for individualized, safe patient care. To improve the quality of care and ensure safe practice, NLN (2010) revised the competencies for graduates of associate degree nursing programs to include nursing judgment, the ability to make judgments in practice, and the spirit of inquiry to challenge the status quo and question underlying assumptions. AACN (2008) defined clinical judgment as the "outcomes of critical thinking in nursing practice" (p. 36).

The nursing process has been used to guide decision-making and actions of nurses since the 1960s (Jones \& Brown, 1991). The American Nurses Association Standards of Practice (2004) states that the nursing process is unique to the discipline of nursing, provides the foundation for clinical decision-making, and includes all the significant actions taken by nurses to provide a competent level of nursing care. The nursing process involves the comprehensive assessment of pertinent patient data, analysis of the data to identify diagnoses or problems, 
identification of expected outcomes individualized to the patient or problem, development of a plan with individualized strategies and alternatives to achieve the outcomes, implementation of the plan, and evaluation of the patientes progress towards achievement of outcomes (American Nurses Association [ANA], 2004). The nursing process provides a common language and structural framework for nurses to think through patient problems (Kataoka-Yahiro \& Saylor, 1994). However, some researchers have suggested that the nursing process is a narrowly focused process that fails to account for the complexity of and many factors that influence clinical judgment; therefore, relying solely on this model is not a sufficient conceptualization of critical thinking (Jones \& Brown, 1991; Kataoka-Yahiro, 1994; Tanner, 2006b). Others recognized a relationship between critical thinking, application of the nursing process, and clinical decisionmaking which provides a systematic framework for nursing care (Alfaro-LeFevre, 1995; Duchscher, 1999; Paul \& Heaslip, 1995; Shirrell, 2008; Su \& Juestel, 2010).

Critical thinking defined. The importance of critical thinking as an essential educational outcome is well documented in the literature (Daly, 2001; Facione, 1990; McMillan, 1987; Paul, 1995). The review of literature indicates there are multiple definitions of critical thinking; a widely accepted single definition is lacking. As a result, there is an absence of clarity of the definition of critical thinking which can result in inconsistencies with the application of the concept (Beyer, 1987; Daly, 1998; Kataoka-Yahiro \& Saylor, 1994; Scheffer \& Rubenfeld, 2000; Turner, 2005). As noted by the authors, this lack of clarity has generated much confusion among researchers, educators, and clinicians

Although theories of critical thinking can be traced back to the teachings of Socrates, John Dewey, a renowned American philosopher and educator, is noted as having a major influence in the development of this concept (Simpson \& Courtney, 2002). Dewey (1916) 
suggested effective thinking is dependent upon one ${ }^{\text {es }}$ past and present experiences which provide the resources to cope with a difficulty. Thinking involves reflection on past experiences from which inferences can be drawn to discover connections between what we do and the consequences that occur. Dewey emphasized thinking was "a method of an educative experience" (Ch. 12, Summary), requiring the learner to have a true situation of experience. Dewey proposed that thinking results from a situation of personal interest to the learner which causes perplexity, confusion, and doubt and from this situation of experience, a real problem develops. As Dewey discussed, the interpretation of observations and the use of past information become the stimulus for thought to deal with the problem. Through careful examination, inspection, exploration, and analysis of these observations and information, the learner develops suggested solutions that can be put into action and tested. Consequences resulting from this experimental testing of solutions provide the learner with clear meaning and opportunity to discover the validity of the solutions. As identified by Dewey, "Thinking includes all of these steps: the sense of a problem, the observation of conditions, the formation and rational elaboration of a suggested conclusion, and the active experimental testing” ( $\mathrm{p}$ 157). As a result, the learner attempts to derive meaning and understanding from these experiences, which results in learning.

Expanding on Dewey see work, Ennis (1962) attempted to present a clear and detailed description of critical thinking that could serve as a basis for further research. Defining critical thinking as the correct assessing of statements, Ennis identified twelve aspects or attitudes required for the three distinct dimensions of critical thinking: logical, criteria, and pragmatic. As described by McPeck (1981), the logical dimension involves knowing the meanings and implications of the words and statements to judge relationships between them. Having 
knowledge of the subject related criteria and standards for judging the statements is defined by McPeck as the criteria dimension. McPeck further defines the pragmatic dimension as the ability to determine when one has enough evidence to judge the statement's purpose and consequences. McPeck (1981), acknowledged the contribution of Ennis ${ }^{e e}$ s work on critical thinking. As McPeck acknowledged, the three dimensions of critical thinking gave significant importance to the setting in which a judgment is made and the practical consequences of such in relation to specialized information and knowledge. However, McPeck challenged Ennis ${ }^{\text {ee }}$ definition of critical thinking, noting it lacked a clear distinction of what he meant by the correct assessing of statements. McPeck did note the acknowledgment by Ennis that a degree of intelligent judgment, in addition to knowing the meaning and applying knowledge, is usually required in the pragmatic dimension. Therefore, McPeck (1981) stated, "Critical thinking does not merely refer to the assessment of statements but includes the thought processes involved in problem solving and active engagement in certain activities" (p. 13).

McPeck (1981) defined critical thinking as the "intelligent use of all available evidence for the solution of some problem" (p. 12) and involves careful and precise thinking about the problem, activity, or subject area. As McPeck noted, it does not take truth for granted, but considers alternative hypotheses and possibilities. McPeck emphasized that critical thinking requires the appropriate use of skepticism, learning to know when and what to question, in addition to the judicious use of skepticism. He further emphasized that critical thinking must be supplemented with detailed knowledge and an understanding of the norms and standards along with experience in the field under consideration. Therefore, McPeck proposed that critical thinking involves the appropriate use of reflective skepticism that requires an understanding of the specific areas of knowledge and skill in question, so as to provide a more satisfactory 
solution to, or insight into, the problem at hand. McPeck recognized that critical thinking is teachable through drills, exercises, or problem solving, but requires both the cognitive and affective domains of a student ${ }^{e c}$ s learning in the area of study.

Paul (1995) defined critical thinking as "thinking about your thinking while you are thinking in order to make your thinking better" (p. 91). He identified the need for self-

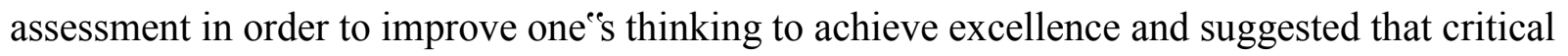
thinking is purposeful thinking occurring only when there are problems to be solved. As Paul discussed, critical thinking is guided by intellectual standards, supports the development of intellectual traits, identifies the elements of thought, and yields a predictable, well-reasoned answer. Intellectual standards important to the critical thinking process were defined by Paul as "the ability to evaluate information for its relevance, accurately identify assumptions, construct plausible inferences, identify relevant points of view, and distinguish significant from insignificant information" (p. 54). Critical thinking, as defined by the National Council for Excellence in Critical Thinking Instruction, is "the intellectually disciplined process of actively and skillfully conceptualizing, applying, analyzing, synthesizing, or evaluating information gathered from, or generated by, observation experience, reflection, reasoning, or communication, as a guide to belief and action" (Paul, 1995, p. 110).

Daly (1998), in an attempt to define critical thinking and its importance to nursing practice, compared the definitions of critical thinking by Ennis (1962), Facione (1984), Halpern (1989), Landis \& Michael (1981), McPeck (1981), and Watson \& Glaser (1991). Daly acknowledged critical thinking to be "purposeful thinking, i.e. selectively attending to something to achieve a goal" (p. 324) which includes an evaluative element. Although there were unique elements in each of the definitions Daly reviewed, commonalities were obvious. He ascertained 
that critical thinking can "be associated with knowledge, cognitive skills, complex reasoning, argumentation, beliefs, action, problem identification, evidence, and the envisioning of alternative frames of reference and possibilities" (p. 325). Daly identified critical thinking as autonomous, purposeful, reasoned thinking that results from the interaction between an individual and information received to determine the authenticity, accuracy and worth of information, knowledge claims, and arguments. Daly did point out that although Landis \& Michael (1981) proposed that critical thinking may provide a greater understanding of a problem, it may not always conclude with solutions to the problem. Therefore, Daly suggested that critical thinking and problem solving may not be completely synonymous.

In response to the diversity of definitions of critical thinking found in the literature and the national movement in the 1980s to infuse critical thinking into the curricula of K-12 and post-secondary institutions, the American Philosophical Association"s (APA) Committee on PreCollege Philosophy asked Peter Facione to lead a systematic inquiry into the current state of critical thinking (Facione, 1990). As noted in this Delphi research project (Facione, 1990), the need to clarify the skills and dispositions that are characteristic of a critical thinker, identify effective ways critical thinking can be taught, and explore ways that critical thinking can be assessed was crucial for the development of this concept into K-12 curricula and college level academic programs. A team of experts, widely recognized for their expertise and experience in critical thinking, formed an interactive panel of experts for the Delphi research project from February 1988 through November 1989. Outcomes of this project resulted in fifteen recommendations pertaining to the instruction and assessment of critical thinking. An APA consensus definition regarding critical thinking can be found in Table 1 of the report. This statement reads: 
We understand critical thinking to be purposeful, self-regulatory judgment which results in interpretation, analysis, evaluation, and inference, as well as explanation of the evidential, conceptual, methodological, criteriological, or contextual considerations upon which that judgment is based. CT is essential as a tool of inquiry. The ideal critical thinker is habitually inquisitive, wellinformed, trustful of reason, open-minded, flexible, fair-minded in evaluation, honest in facing personal biases, prudent in making judgments, willing to reconsider, clear about issues, orderly in complex matters, diligent in seeking relevant information, reasonable in the selection of criteria, focused in inquiry, and persistent in seeking results which are as precise as the subject and the circumstances of inquiry permit. (p. 2)

Critical thinking as defined by nursing. Literature reviews of nursing education have also identified a lack of a consensual definition of critical thinking specific to the discipline of nursing (Turner, 2005). This lack of a common definition has led to some confusion and uncertainty when academic programs attempt to define and measure the concept in their curricula (Hicks, 2001; Jones \& Brown, 1991; Kataoka-Yahiro \& Saylor, 1994; Scheffer \& Rubenfeld, 2000; Simpson \& Courtney, 2002; Turner, 2005). In attempts to reach a more comprehensive understanding of critical thinking in nursing, a Delphi study was conducted between 1995 and 1998 with an international panel of nursing experts from nine countries and 23 states in the United States (Scheffer \& Rubenfeld, 2000). As a result of this Delphi technique, a consensual definition of critical thinking was achieved that included the cognitive skills of thinking, as well as the affective component of critical thinking required for nursing. Identified by Scheffer $\&$ Rubenfeld (2000), the consensus statement reads: 
Critical thinking in nursing is an essential component of professional accountability and quality nursing care. Critical thinkers in nursing exhibit these habits of the mind: confidence, contextual perspective, creativity, flexibility, inquisitiveness, intellectual integrity, intuition, open-mindedness, perseverance, and reflection. Critical thinkers in nursing practice the cognitive skills of analyzing, applying standards, discriminating, information seeking, logical reasoning, predicting and transforming knowledge. (p. 357)

As Scheffer \& Rubenfeld pointed out, this Delphi study emphasized the importance of including the affective component of critical thinking, in addition to the cognitive component, through the defined habits of mind. As they further noted, creativity and intuition were included as important affective components required for critical thinking in nursing.

Turner (2005) conducted a review of nursing literature from 1982-2002 to determine changes in the concept of critical thinking over time and how it applied to nursing education and nursing practice. Although she noted there were a variety of definitions of critical thinking found in the literature, more recent literature referenced the APA Delphi report's definition of critical thinking. In addition, the literature review revealed that critical thinking was often used interchangeably with nursing process, clinical reasoning, clinical decision-making, clinical judgment, and problem solving. Critical thinking in nursing, as defined by Turner (2005), is a purposeful, self-regulating judgment that requires knowledge in the area of one"s thinking and is characterized by the most common attributes of analysis, reasoning, inference, interpretation, knowledge, and open-mindedness. Tanner further notes that critical thinking is associated with improved decision-making, clinical judgments, and problem solving, resulting in safe, competent practice. 
Characteristics of the critical thinker. For nurses to become effective critical thinkers, they must have a body of nursing knowledge based on the concepts addressed in science, humanities, and nursing courses (Kataoka-Yahiro \& Saylor, 1994). As Kataoka-Yahiro and Saylor (1994) noted, without a knowledge base in nursing, one is not able to think about nursing problems and identify appropriate actions or solutions for those problems. The importance of the nurse in exhibiting both cognitive skills and affective dispositions (habits of mind) for effective critical thinking is well supported in the literature (Facione \& Facione, 1997; Heaslip, 1993/2008; Ignatavicius, 2001; Paul, 1995; Scheffer \& Rubenfeld, 2000; Simpson \& Courtney, 2002).

Six essential cognitive skills used by expert critical thinkers include interpretation, analysis, evaluation, inference, explanation, and self-regulation (Facione, 1990; Facione \& Facione, 1996; Ignatavicius, 2001). As defined by Ignatavicius (2001), interpretation involves clarifying and understanding the meaning of patient data. Patient problems are identified through analysis; the process of evaluation identifies expected patient outcomes and assesses the degree to which they have been met. Inference results in drawing conclusions about the patient $t^{\text {ee }}$ health status, explanation refers to the ability to justify actions, and self-regulation involves the ability to justify, correct, or improve one ${ }^{e e}$ sactions (p. 32).

Affective dispositions, also referred to as attributes or habits of mind, are the personal traits that characterize good critical thinkers (Facione, 1990). These attributes include truthseeking, open-mindedness, analyticity, systematicity, self-confidence in reasoning, inquisitiveness, and cognitive maturity (Facione \& Facione, 1997). As Facione and Facione (1996) indicated, the ideal critical thinker demonstrates intellectual honesty; is eager and curious to find the best knowledge in the context of the situation; is objective, unbiased, and open to new 
ideas and different views; is willing to reconsider; utilizes reasoning and evidence in problem identification and anticipation of consequences; approaches complex problems with organization, persistence, and diligence; trusts one"s own reasoning skills; and exhibits caution in making, suspending, or revising judgments. Ignatavicius (2001) identified several attributes and traits that are characteristic of an expert thinker in nursing. For nurses to develop into expert critical thinkers, Ignatavicius acknowledged the importance of continuous learning and expansion of knowledge. Additionally, she noted that nurses must remain focused on patient outcomes, become risk takers, and think out of the box to explore new and creative ways to solve problems and meet the needs of patients.

Critical thinking, clinical judgment, and clinical reasoning. The association of critical thinking and clinical judgment, an essential skill of every health care professional (Prion, 2008; Tanner, 2006b, White, 2003), is apparent in the literature. Alfaro-LeFevre (1995) identified the significance of critical thinking in making clinical judgments in nursing and defined clinical judgment as "critical thinking in the clinical area" (p. 46). Critical thinking is further defined by Alfaro-LeFevre as the meaningful thinking nurses use to make decisions based on evidence, not assumptions. Facione and Facione (2008) described the relationship of critical thinking and clinical judgment as "Critical thinking is the process used to make a judgment about what to believe and what to do about the symptoms our patient is presenting for diagnosis and treatment" (p. 2). Tanner (2006b) defined clinical judgment as the conclusion drawn about a patient"s needs or problems, the decision of what actions to take, and the modification or use of standard or new approaches appropriate for the patient. Tanner further defined clinical reasoning as the "processes by which nurses and other clinicians make their judgments" (p. 204). 
As defined by Paul (1995), reasoning "is the art of figuring things out for yourself" (p. 305). Clinical reasoning, also referred to as diagnostic reasoning, is an essential component of nursing practice that is required for nurses to "figure out" a patient"s health status, identify patient problems accurately, make decisions about patient care, and form clinical judgments (O'Neill \& Dluhy, 1997; Simmons, Lanuza, Fonteyn, Hicks, \& Holm, 2003). Development of clinical reasoning increases the accuracy of decisions, thus improving patient outcomes (Simmons et al., 2003). Effective clinical reasoning requires the development of both critical thinking (cognitive skill) and reflective thinking (metacognitive skill) (Kuiper \& Pesut, 2004). This is also supported by Alfaro-LeFevre (2004), who stated that critical thinking requires "reasonable, reflective thinking" (p. 9). Meta-cognitive reflection of what one is doing and why is essential to the critical thinking process and improves cognitive thinking skills necessary for the development of critical thinking (Kuiper \& Pesut, 2004). Reflective thinking is essential for the development of the clinical reasoning skills that are necessary for nurses to form clinical judgments (Facione \& Facione, 1996; Kuiper \& Pesut, 2004; Murphy, 2004).

\section{Learning and Acquisition of Knowledge}

Experiential learning. Learning is a process of acquiring skills and knowledge through experience (Bigge \& Shermis, 2004). The importance of experience in the process of learning and knowledge attainment is evident in Kolb"s experiential learning theory. Kolb (1984) defined learning as "the process whereby knowledge is created through the transformation of experience" (p. 38). Based on the works of Dewey, Lewin, and Piaget, Kolb suggested that learning is a continuous, life-long process of creating knowledge that results from the transaction between the objective (environmental) and subjective (personal) conditions of experience. He further discussed the resolution of conflicts between concrete (real-life) experiences and abstract 
concepts and conflicts between observation and action that are required for learning. Kolb proposed that knowledge, skills, and attitudes are gained through four modes of experiential learning. These four modes, described by Kolb, require the learner to become fully involved and without bias to new experiences (concrete experience), reflect on experiences from many perspectives (reflective observation), look for patterns and meanings to create concepts into logically sound theories (abstract conceptualization), and use these theories to make decisions and solve problems (action).

Knowledge gained through learning is increased when students are actively engaged in experiential problem-solving and decision-making as compared to didactic, passive learning (Dewey, 1933; Kolb, 1984). Learning is enhanced when students learn by doing in an environment that replicates a real life situation while faculty serve as a facilitator (Kolb, 1984; Schön, 1987) and role model of critical thinking (Duchscher, 2003; Ironside, 1999; Paul \& Heaslip, 1995). Experience based education, such as apprenticeships, internships, field placements, work/study programs, gaming simulations, and laboratory studies, has become widely accepted as a method of instruction in colleges and universities (Kolb, 1984).

Situated learning. Based on the situated cognition theory of Brown, Collins, and Duguid, situated learning involves acquisition of skills and knowledge learned in contexts that mirror how the knowledge and skills will be used in real life (Brill, 2001). Brill (2001) emphasized that instructional strategies based on the situated cognition theory require the learner to be actively engaged in an environment that resembles context in the subject matter to be learned, thus making the learning more useful, relevant, and transferable. As suggested by Brill, learning that occurs as a result of strategies that apply the principles of situated cognition theory teach students the conditions for applying knowledge, the ability to see the implications of 
knowledge, and the ability to structure the knowledge learned for future use. Summarized in an article by Stein (1998), outcomes of situated learning identified by McLellan (1996) included: acting on situations, producing negotiated meaning, reasoning, resolving emergent dilemmas, and solving problems. As Stein noted, providing opportunities for students to live subject matter in real-world experiences facilitate the transfer of knowledge from the classroom to practice.

As discussed by Stein (1998), situated learning is a social process occurring within an environment that closely resembles the practice environment and involves the transaction with others. Four elements of situated learning were defined by Stein: content, context, community of practice, and participation. As he discussed, higher order thinking skills required for application of content are emphasized with situated learning. Context provides the setting for the learner to live the experience, while community provides the social interaction to interpret, reflect, and form meaning through dialogue with others. Shared knowledge occurs through the participation and active engagement of the participants (Stein, 1998).

Experiential and situated learning as it applies to nursing. Contextualized experience lies at the heart of professional learning (Kneebone, Scott, Darzi, \& Horrocks, 2004). In nursing, pedagogies of contextualization are used in clinical education to provide opportunity for the transfer of knowledge from theory to practice (Benner et al., 2010). Benner et al. (2010) discussed the benefits of situated learning experiences and recognized that knowledge occurs when the student is immersed in experiences of caring for specific patients in a particular situation. They further noted that the opportunity for understanding a patientes response in relation to the circumstances and events surrounding each unique patient teaches the student to look at the whole situation. Recognizing differences in patient responses within the context of the patient"s situation will lead to individualized patient care and avoid the trap of stereotyping 
patients. As identified by Benner et al., the development of clinical reasoning skills and clinical judgment occurs as students extend their thinking when responding to patient changes and determining what is most important for a particular patient.

A review of qualitative studies in nursing emphasizes the significance of experiential and contextual factors on the development of the critical thinking process (Hicks, 2001). As noted by Del Bueno (2005), "Nursing is a practice art that requires the use of knowledge within a specific set of circumstances" (p. 281) and it is through the experiential and situated learning experiences provided through the nurse-patient interactions that critical thinking and nursing knowledge evolve. However, reflection or conscious thought of the situation is vital for situated learning to occur (McLellan, 1996).

\section{Reflective Thinking as it Relates to Critical Thinking and Knowledge}

Reflective thinking defined. Reflective thinking, which can be traced back to the work of Dewey $(1916,1933)$, is defined as careful examination of issues of concern related to an experience that draws upon knowledge learned from past experiences (Kuiper \& Pesut, 2004). According to Dewey (1933), situations that cause mental difficulty, perplexity, or doubt cause one to draw upon past experiences to find ideas or suggestions to resolve the perplexity or doubt. Dewey proposed that the learner collects additional pertinent data in attempts to understand and explain the situation when faced with a difficult situation. Through active reflection on past experiences, new knowledge is gained that can be used to understand and effectively deal with situations that follow.

Schön (1983), well known for his work on reflective thinking, identifies two types of reflection: reflection-in-action and reflection-on-action. As described by Schön, reflection-inaction refers to the reflective thinking that occurs while one is engaged in a situation of practice 
which an individual is attempting to deal with. The individual uses knowledge from past experiences in attempts to understand the current situation and respond. Reflection-on-action, as defined by Schön, occurs after the situation has taken place in attempts to critique and understand the situation so new knowledge can be applied to future practices. Schön emphasized the role of practice as being important for the development of professional knowledge.

Reflective thinking and relevance to nursing. Experiential learning occurs through active reflection of one ${ }^{\text {ee }}$ own experiences (Kolb, 1984). Knowledge gained through reflective and critical thinking occurs with each clinical experience of practice; however, for learning to occur as a result of reflective thinking, the learner must be actively involved in the clinical experience (Dewey, 1933; Kolb, 1984; Schön, 1983; Teekman, 2000). The learning that occurs by doing can be transferred to different settings without conscious awareness (Schön, 1983). Repeated and successful experiences of practice contribute to learning and add to the knowledge base of one $e^{\text {ee }}$ practice (Duchsher, 1999; Kuiper \& Pesut, 2004; Paul \& Heaslip, 1995; Schön, 1983; Teekman, 2000), improve critical thinking and decision-making (Angel, Duffy, \& Belyea, 2000; Ironside, 1999; Martin, 2002), and are necessary for the development of clinical judgment for future situations (Benner, 2004; Tanner, 2006b).

The NCSBN (2005) recognized the importance of experience and reflection in the development of critical thinking and defined critical thinking as "the intellectually disciplined process of actively and skillfully conceptualizing, applying, analyzing, synthesizing and/or evaluation of information gathered from, or generated by, observation, experience, reflection, reasoning or communications, as a guide to belief and action" (p. 2). Benner (2000) further supported the importance of experiential learning and reflection for the development of good clinical judgments. 
Teekman (2000), in an attempt to study reflective thinking in actual nursing practice, interviewed ten registered nurses for the presence of reflective thinking. As Teekman discussed, reflective thinking, used during situations of doubt or perplexity, required nurses to focus on the situation and choose from options they considered to be the most appropriate for effective action. Previous experiences in practice and collegial support were identified by Teekman as the most important factors affecting the nurses" ability to reflect and understand the situation. In addition, past experiences were identified as providing the base of knowledge that could be drawn upon for clinical decision-making. Furthermore, guidance throughout the reflective process assists the student with the development of reasoning skills and the application of theory to practice (Benner, 2004; Bjork \& Kirkevold, 1999; Murphy, 2004; Tanner, 2006b).

The ability to reflect in practice was identified by Kuiper and Pesut (2004) as being beneficial for learning and increasing knowledge; however, the authors cautioned that reflection takes time to develop. As noted by Kuiper and Pesut, the type of clinical experience may be more important in one"s ability to reflect and gain knowledge than the number of years of experience in practice. Through a review of the literature by Kuiper and Pesut, evidence supported that reflective thinking developed skills which can increase confidence, promoted intellectual growth, fostered responsibility and accountability (Kuiper \& Pesut, 2004), assisted with applying theory to practice (Kuiper \& Pesut, 2004; Murphy, 2004; Ruth-Sahd, 2003), and directly related to improved patient outcomes (Pesut \& Herman, 1999).

\section{Critical Thinking and Knowledge as Outcomes of Nursing Education}

The goal of pre-licensure nursing educational programs is to prepare graduates with the knowledge and skills necessary for safe entry into professional nursing practice (NCSBN, 2011). Knowledge required for safe practice requires more than simply memorizing, recalling, or 
understanding content learned in nursing and the biological and social sciences. Nursing is a practice art that requires the application of content for decision-making and clinical judgments in clinical situations (Rauen, 2001). Nursing judgment requires the nurse to use the cognitive skills of critical thinking for the application, analysis, and synthesis of knowledge (Del Bueno, 2005). Therefore, nursing education programs must ensure that graduates have not only the disciplinespecific knowledge of nursing, but also the critical thinking skills required for the application, analysis, and synthesis of this knowledge (Rauen, 2001).

Critical thinking as an outcome. In response to accreditation requirements, the complexity of health care environments that require higher level thinking skills, and the expectation that nurses will be prepared to practice safely and competently, critical thinking has become an expected educational outcome and a key component for evaluation of nursing programs (AACN, 2008; Boland, 2009; Daly, 1998; Hicks, 2001; Jones \& Brown, 1991; Patterson, Crooks, \& Lunyk-Child, 2002; Paul, 1995; Simpson \& Courtney, 2002; Su \& Juestel, 2010; Turner, 2005). Critical thinking skills, essential for effective clinical reasoning, clinical judgment, and problem solving, are required for nurses to respond efficiently and effectively to the wide range of patient situations they may encounter. The lives of patients depend on the nurses $^{\text {ee }}$ ability to form critical judgments, make quick decisions, and take proper action (Rauen, 2001). Professional nurses must learn to "think like a nurse" (Heaslip, 1993/2008). As noted by Kataoka-Yahiro \& Saylor (1994), "The characteristic that distinguishes a professional nurse is cognitive rather than psychomotor ability" (p. 351).

Critical thinking and development of knowledge. Critical thinking facilitates the gradual development of expert knowledge and is needed to contextualize and adjust what the nurse knows to each particular case (Facione \& Facione, 1996; Paul \& Heaslip (1995). As Paul 
and Heaslip cautioned, with the uniqueness of each patient and variations in human responses to illness, it is important that nurses do not fall into the trap of using standardized care plans and nursing practice inappropriately. As the authors noted, mindlessly doing tasks to complete nursing care without taking into consideration the unique differences and needs of each patient may result in incomplete, substandard care. To provide holistic care, nurses must use critical thinking to effectively analyze and apply their nursing knowledge to individual cases.

As Daly (1998) noted, the practice of nursing requires the application of appropriate nursing knowledge to analyze and construct arguments when caring for unique patients in constantly changing environments. Critical thinking, defined as "a nonlinear, recursive process in which a person forms a judgment about what to believe or what to do in a given context," (Facione \& Facione, 1996, p. 131) recognizes the importance of being able to utilize multiple critical thinking skills for problem solving. It is important that nurses are able to recognize and analyze the relevant context or set of circumstances surrounding the clinical decisions they make (Daly, 1998; Facione \& Facione, 1996). Daly further acknowledged that nurses must be able to recognize when circumstances surrounding a patient problem may render an intervention or procedure inappropriate in respect to the anticipated outcome of a problem. Patient specific factors or circumstances, identified by Daly, that may affect a nurse es clinical decisions include: age, gender, ethnicity, religion, culture, socio-economic status, cognitive ability and level of development, physical ability, disease process, and practice boundaries. The importance of the context in relationship to critical thinking is further supported by the APA ${ }^{\text {es }}$ consensus statement (1990) that emphasizes the importance of taking into consideration the evidence, interpretation of observations, underlying principles and accepted standards, as well as the context when one uses critical thinking skills to form a purposeful judgment. The search for the best knowledge in 
a given context is central to the development of nursing knowledge and requires openmindedness and a tolerance of multiple perspectives and interpretations when supported by evidence (Facione \& Facione, 1996).

Measurement of knowledge and critical thinking. For graduates to practice as registered nurses, they must obtain a license to engage in nursing practice (NCSBN, 2011). As discussed on the NCSBN (2011) website, licensure of the nurse by each state"s Board of Nursing ensures the public that the nurse has minimal, entry level competency to perform the scope of nursing practice. To obtain a license to practice, the individual must graduate from a nursing education program approved by a state board of nursing or a state commission on higher education and pass the National Council Licensure Exam for Registered Professional Nurses (NCLEX-RN). This exam is a secure, high-stakes, psychometrically sound examination to determine if an individual has the minimal competency needed to practice nursing safely (NCSBN, 2011). As the NCSBN acknowledged, the NCLEX-RN test plan is based upon practice analysis studies, conducted every three years, which identify activities expected of newly licensed nurses. The test, which largely consists of single-answer, four-option, multiplechoice items, is designed to measure the test taker"s snowledge and ability to use critical thinking when solving nursing problems (Morrison, Nibert, \& Flick, 2006).

With the NCLEX-RN being the accepted measurement of knowledge required for entry level practice, NCLEX-RN passage rates are used as one outcome assessment for the evaluation and accreditation of nursing programs. Standard 6 of the NLNAC Accreditation Manual (2008) requires nursing programs to evaluate student learning for the purpose of determining if outcomes of the nursing education unit have been achieved. Performance on licensure exam, Standard 6.5.1 program outcome, requires the nursing program to demonstrate that the licensure 
exam pass rates will be at or above the national mean. The West Virginia Board of Examiners for Registered Professional Nurses requires schools of nursing to achieve minimally an $80 \%$ pass rate.

With high stakes associated with NCLEX-RN passage rates, most schools of nursing rely on multiple-choice exams as the assessment of the student ${ }^{\mathrm{ee}} \mathrm{s}$ knowledge of nursing content and concepts (Ironside, 2005; Morrison et al., 2006). Facione and Facione (2008) proposed that measurement of critical thinking skills can be achieved with multiple choice and short answer essay tests. However, the use of multiple choice exams as an assessment of student learning is being questioned, but nursing programs continue to place a huge focus on strategies to answer multiple-choice questions to prepare students for NCLEX (Benner et al., 2010). As Del Bueno (2005) noted, the ability to select the correct answer of a multiple-choice question does not equate to clinical judgment. She further noted that in actual practice, patients do not provide the nurse with a list of options that describe their symptoms, identify possible complications, or suggest possible solutions. However, Del Bueno did acknowledge that the use of logical reasoning and application of knowledge can help the student decide which answers are correct. Paul (1995) acknowledged that multiple choice tests often used today are designed to measure rote memorization. He emphasized that for effective measurement of learning, the assessment tool must test the student"s use of higher order reasoning skills in authentic environments that the student will be required to perform in. Paul further supported that standard multiple-choice items can be used to test "authentic skills, abilities, and dispositions in authentic contexts" (p. 117). Multiple-choice exams are also supported by Facione \& Facione (2008), who claim that multiple choice questions can be written that require the application of critical thinking skills for 
problem solving, the evaluation of the quality of the solution, and an understanding of the rationale for the solution.

Health Education Systems, Inc. (HESI) is well known for their specialty and comprehensive exit examinations designed to evaluate student learning and predict student success on the NLCEX-RN. The conceptual framework and test-writing model used by HESI in the construction of their exams are based on Paul 's critical thinking theory and Bloom ${ }^{e e}$ Taxonomy of Educational Objectives for Cognitive Domain (Morrison et al., 2006). As Morrison et al. (2006) noted, every exam designed by HESI incorporates critical thinking as a concept. The test-writing model used for developing test items that promote critical thinking incorporates four criteria that must be met when writing a multiple-choice question: rationale included for each test item, questions written at the application level or above, multi-logical thinking required to answer, and a high level of discrimination required to choose among plausible alternatives (Morrison \& Free, 2001).

Revised in 2001, Bloom 's taxonomy provides a model of classifying thinking according to six cognitive levels of complexity: remembering, understanding, applying, analyzing, evaluating, and creating (Forehand, 2005). Explained by Forehand (2005), remembering, the lowest level of thinking, involves the recall of relevant knowledge from long term memory. The second level, understanding, refers to the ability to construct meaning of messages through interpreting, classifying, organizing, summarizing, comparing, and inferring. Applying is defined as the ability to carry out a procedure through implementation and analyzing refers to the ability to break information apart and determine how the different parts relate. Evaluating, the highest level of thinking, is the ability to make judgments based on standards; creating refers to the ability to put elements together to form a whole and reorganize elements into a new pattern. 
Paul (1995) proposed that the process of critical thinking occurs at the higher levels of Bloom 's taxonomy, beginning at the level of applying information. Based on Paul"es definitions of critical thinking, HESI defines critical thinking as "The process of analyzing and understanding how and why a certain conclusion was reached" (Morrison et al., 2006, p. 11). As Morrison et al. (2006) explained, Bloom "s Taxonomy is used as a means of understanding how information is processed and the level of thinking required to ensure test items are written at the application level or above. They further proposed that questions written at the higher cognitive levels of applying, analyzing, evaluating, and creating, that require multi-logical thinking to answer, evaluate a higher level of knowledge beyond memorization and recall. These test items require the student to logically and systematically apply knowledge of course content to practice situations and evaluate the student"s ability to think critically and make sound judgments to solve clinical problems within the context of nursing practice (Morrison et al., 2006).

Controversy exists in the literature regarding the transferability of critical thinking skills from one discipline to another and the best tool for measuring critical thinking abilities of nursing students (Morrison \& Free, 2001). McPeck (1981) emphasized that critical thinking is discipline-specific and must be taught and measured within the context of a specific discipline. Many commercially prepared standardized critical thinking exams, such as the Watson-Glaser Critical Thinking Assessment (WGCTA) and the California Critical Thinking Test (CCTT), are not specific to nursing and have not been effective in demonstrating improvements in critical thinking skills of nursing students (Brunt, 2005; Morrison \& Free, 2001; Simpson \& Courtney, 2002). Many educators believe that measurement of critical thinking in nursing needs to be evaluated within the context of nursing (Brunt, 2005). Development of a critical thinking instrument to measure critical thinking in the context of nursing practice would provide a more 
consistent method of assessing students and advancing research-based practice (Seymour, Kinn, \& Sutherland, 2003). As identified by Brunt (2005), research on the development of critical thinking in nursing practice using nursing-specific tools is needed.

For the purpose of this study, the HESI exit exam (HESI E ${ }^{2}$ ) was used as the tool to measure nursing knowledge and critical thinking. This exam was chosen due to the effectiveness of the exam in measuring the knowledge, skills, and constructs for entry-level nursing practice as reflected in the NCLEX-RN test blueprint. As noted earlier, test items on the exam are designed to evaluate critical thinking skills of the student within the discipline of nursing. In addition to providing subject area scores and a composite score that assesses the knowledge required for entry level practice, a critical thinking score is provided that measures the studentes higher order thinking skills for the decision-making and clinical judgment required within the context of nursing practice. The HESI exit exam is discussed more thoroughly in chapter three.

Development of critical thinking. It has been well established that critical thinking is vital for clinical decision-making and clinical reasoning essential for making sound clinical judgments necessary for safe, competent practice. Being cognizant of this, nurse educators must be committed to developing critical thinking abilities of their graduates (Daly, 2001; Del Bueno, 2005; IOM, 2003; Ironside, 2005; Kataoka-Yahiro \& Saylor, 1994; Paul \& Heaslip, 1995; Turner, 2005). Employers expect nurses to have the necessary critical thinking abilities to accurately identify patient problems, prioritize and effectively manage problems in a relevant time period, and support clinical decisions and judgments about patient care (Del Bueno, 2005; Hicks, 2001; Jeffries, 2008; Walker \& Redman, 1999). Failure to do so may result in unsafe care. However, Del Bueno (2005) reported that only 35\% of new graduates, regardless of their educational preparation, are prepared with the critical thinking skills required for entry level 
clinical judgment. Therefore, nurse educators are challenged to look at how they teach the critical thinking skills necessary for the development of clinical judgment. New pedagogies that are more effective in fostering these thinking skills must be explored.

Studies have shown that experience, time for reflection, and processing of information have a positive correlation on the development of critical thinking skills (Benner, 2004; Duchscher, 2003; Martin, 2002; O'Neill \& Dluhy, 1997; Rapps, Riegel, \& Glaser, 2001). In a review of the literature, a major theme that emerged from qualitative studies of critical thinking suggests that critical thinking is an unfolding process that develops over time and evolves from the contextual experiences of nurse-patient interactions (Hicks, 2001). Oermann (1997) also emphasized that critical thinking develops over time through a variety of experiences. Shin, Lee, Ha, and Kim (2006) utilized the California Critical Thinking Disposition Inventory (CCTDI) to measure students ${ }^{\text {ee }}$ disposition to critical thinking as they progressed through a baccalaureate nursing program. Results of the study supported that critical thinking skills increased significantly as students progressed through academic years.

In a qualitative study that analyzed the critical thinking processes of baccalaureate nursing students, Sedlak (1997) found that a supportive environment and opportunity for discussion of experiences had a positive influence on the development of a student" thinking abilities. The importance of reflection on the development of critical thinking skills was further supported by research conducted by Murphy (2004). Haffer and Raingruber (1998) identified confidence as being important in the development of critical thinking. The importance of self-confidence and a supportive environment as factors facilitating the development of critical thinking was also supported by research conducted by Hagbaghery, Salsali, and Ahmadi (2004) and White (2003). 
Strategies to promote critical thinking. Learning that occurs from reflective thinking requires both active involvement of the learner and a clinical environment supportive of the learner's needs (Teekman, 2000). Active involvement increases one ${ }^{e e}$ s interest in learning and motivation for learning (Cioffi, 2001). The environment in which the experience takes place can facilitate or hinder critical thinking. Environments that reinforce memorizing and retention of facts, demand perfection, or reinforce the status quo impede the development of critical thinking abilities (Kataoka-Yahiro \& Saylor, 1994). Dialogical situations, requiring one to express views

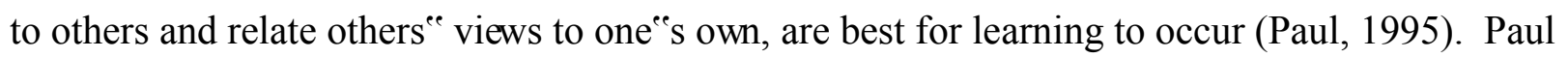
(1995) suggested that didactic teaching, such as lecturing, encourages monological linear thinking, which results in learning through memorization and recall. He further suggested that the acquisition of knowledge implies that the student has an understanding and comprehension of information.

In an attempt to ensure that all content is covered, nurse educators typically rely on the lecture format to deliver large amounts of content. This lecture format often lacks an interactive environment that focuses on the application of knowledge (Benner et al., 2010; Del Bueno, 2005; Ironside, 2005). Paul and Heaslip (1995) cautioned educators to avoid the trap of trying to cover large amounts of content and suggested that educators focus on the most basic, central concepts. Paul and Heaslip reinforced that student attempts to memorize large amounts of information can lead to a temporary and superficial retention of knowledge. Active engagement of the learner in experiential and situated teaching pedagogies increases not only retention of content, but also provides a deeper understanding of the significance of content in different contexts, necessary for clinical judgment (Ironside, 2005; Tanner, 2008). Therefore, new teaching pedagogies are needed to shift the focus away from memorization and recall of vast amounts of content and 
acquisition of skills to a focus on teaching the thinking skills required for practice in complex health care situations (Ironside, 2005; Paul \& Heaslip, 1995).

The development of critical thinking occurs through techniques of instruction that permit the student to actively practice critical thinking skills (Rauen, 2001; Schank, 1990). Students must have the opportunity to analyze information and determine the relevance to practice; therefore, activities and assignments should be designed so that students must think their way through them (Paul \& Heaslip, 1995). As Paul \& Heaslip (1995) pointed out, knowledge learned from rote memorization results in rule-driven practice that limits the ability of the student to contextualize and use reasoning to form accurate clinical judgments. Providing opportunities for students to critically reason their way through the large body of information, provided to them for appropriate application to practice, will lead to a deeper understanding of patient situations (Brooks \& Shepherd, 1990; Paul \& Heaslip, 1995).

In a review of the literature, instructional strategies designed to promote active methods of learning critical thinking and application of information include: role playing, small group activities, case studies, simulations, journals, and questioning (Rowles \& Russo, 2009; Shin et al., 2006; Simpson \& Courtney, 2002). Restructuring of curricula to include case-based pedagogies and problem-based learning has been seen to improve clinical reasoning skills and dispositions (Facione \& Facione, 2008) and to assist with linking classroom theory to clinical practice (Chappy, Jambunathan, \& Marnocha, 2010).

Problem-based learning (PBL), based on the situated cognition theory, has been associated with higher levels of critical thinking, decision-making, problems solving, and communication skills (Holaday \& Buckley, 2008; Niemer, Pfendt \& Gers, 2010; Simpson \& Courtney, 2002). This teaching strategy provides unfolding case studies of real practice 
situations that require students to identify problems, seek solutions, prioritize and adjust care as the patient"s condition changes over time (Rowles \& Russo, 2009). PBL provides opportunity for students to actively engage in group work and practice clinical reasoning skills as they learn to think or reason through changes in the patient ${ }^{\text {ee }}$ s condition (Benner et al., 2010; Rowles \& Russo, 2009). Working in groups to solve problems provides opportunity for students to become tolerant of the ideas of others (open-mindedness) and evaluate conflicting ideas as they seek to find the best solution (truth-seeking) (Ozturk, Muslu \& Dicle, 2008). Research, comparing the effects of PBL and lecturing on the development of critical thinking scores, supports that PBL is more effective in developing critical thinking skills. Findings of two studies by Tiwari, Lai, So, and Yuen (2006) and Ozturk et al. (2008) demonstrated significant improvements in critical thinking dispositions, as measured by the CCTDI, for those students taught by the PBL model compared to those taught by the traditional lecture model.

Perceptions of the student-teacher relationship can influence the development of critical thinking and it is important that sharing of experiences with faculty and peers is conducted in a supportive, non-judgmental environment (Davies, 1995; Tanda \& Denham, 2009). Faculty guidance in assisting students to reflect upon actions can help students develop the skills of critical thinking (Facione \& Facione, 1996). It is important that the guidance occurs in a safe climate of mutual respect and support that permits and ensures confidentiality of expression of feelings (Kuiper \& Pesut, 2004; Ruth-Sahd, 2003). Paul and Heaslip (1995) recognized the importance of instructors thinking out loud as they work through nursing problems to provide a modeling of the critical thinking process. In addition, the authors suggested that students are provided opportunities to reason out loud as they work through solutions to nursing problems. Recommendation 14 of the APA Delphi research (Facione, 1990) recommends that "Teaching 
CT is most effective if the instructor models CT dispositions and the proper use of CT skills in the very process of instruction" (p. 17).

White (2003) investigated how fourth-year nursing students learned clinical decisionmaking. Five components essential for learning clinical decision-making emerged from the study: gaining confidence in skills, building relationships with staff, connecting with patients, gaining comfort with self, and understanding the clinical picture. White found that confidence in technical and communication skills permitted students to focus on the clinical situation and patient needs as opposed to their own needs and the anxiety often associated with lack of confidence. White further found that learning was enhanced and confidence increased when the students perceived the nurses to have confidence in them. White concluded that nurses who were willing to describe their thought processes while providing care helped to facilitate the student $^{\text {ee }}$ s clinical decision-making process. In addition, it was determined that as the student gained experience, self-confidence, and knowledge, understanding of the clinical picture and recognizing patterns to assist the student in anticipating patient needs were developed.

Twibell, Ryan, and Hermiz (2005) explored perceptions of nursing faculty in teaching critical thinking. Faculty identified the core nature of critical thinking as "putting it all together through information seeking, reflecting, assigning meaning, problem solving, predicting, planning, and applying information" (Twibell, Ryan, \& Hermiz, 2005, p. 71). Research conducted by Hagbaghery et al. (2004) used interviewing and observation to identify factors affecting clinical functioning and clinical decision-making of 26 nurses with more than five years of experience. Being self- confident and feeling competent were identified by the authors as the two most important factors influencing clinical decision-making. 
Regularly questioning students using the Socratic Method that challenges thinking and encourages explorations of differing or opposing points of view can enhance the development of critical thinking (Paul \& Heaslip, 1995). Twibell et al. (2005) suggested that critical thinking is best taught in clinical settings. Strategies for teaching critical thinking in the clinical environment, as identified by Twibell et al., include: questioning by instructors, written feedback on nursing care plans, clinical case studies, clinical conferences, and journaling.

\section{Nursing Education}

The curricula of today's nursing education programs traditionally consist of classroom instruction, planned laboratory experiences in skills laboratories, and clinical experiences in health care settings with patients (Benner et al., 2010). As Benner et al. (2010) discussed, nursing theory is presented in a classroom setting and psychomotor skills are taught and practiced in a skills laboratory. In the clinical setting, the student is expected to apply the knowledge and skills when caring for patients. With this model, patients were at the mercy of inexperienced students as they practiced the clinical and psychomotor skills learned in the laboratory setting (Harder, 2009). Placing inexperienced students in the clinical setting, who do not have the necessary knowledge and problem solving skills to provide safe nursing care, has the potential to cause harm to patients (Weis \& Guyton-Simmons, 1998). Students reported feelings of anxiety and fear of making mistakes in clinical placements, especially with initial and new clinical areas (McCallum, 2007).

As identified by many nurse experts, this approach to nursing education creates a sharp divide between classroom theory and clinical experiences where the application of theory occurs. Methods of teaching theory in the classroom typically lack pedagogical strategies of experiential learning that provide application of knowledge and active involvement of the learner, making it 
difficult for students to apply theory in the clinical setting (Benner et al., 2010). Students, faculty, and graduates have identified the need for more connection between what is taught in the classroom and clinical practice (Benner et al., 2010; Henneman \& Cunningham, 2005; Jacobson \& Grindel, 2006).

Del Bueno (2005) found the majority of new graduates of nursing programs have difficulty translating knowledge into practice and as few as 35 percent of new graduates meet entry expectations for clinical judgment. Graduates frequently reported that having more clinical time would have better prepared them for transition into practice (Chappy et al., 2010; Pellico et al., 2009). Garrett (2005) examined student nurse perceptions of clinical decision-making in the final year of study. Students linked clinical experiences, rather than their cognitive ability, with their level of skill in clinical decision-making. Educators are challenged to use innovative approaches for more effective integration of classroom knowledge and clinical experiences to better prepare their graduates with the reasoning skills required for practice (Benner et al., 2010; IOM, 2011; Nehring \& Lashley, 2010; NLN, 2005b; Tanner, 2008).

Traditional clinical education. The most common model of clinical education used today places students directly in health care environments to provide hands-on care for one or two patients (Ironside \& McNelis, 2010; Nehring, 2008; Tanner, 2006a). As noted by Ironside and McNelis (2010), typically one faculty member is assigned to a group of 8-12 students, with the size of the group frequently being mandated by state boards of nursing. This model of clinical education, supported by experiential learning theory, has been identified as one of the strengths of nursing education (Benner et al., 2010) and assists in the development of a student" $\mathrm{s}$ clinical decision-making skills (Tanda \& Denham, 2009). 
The NLN Task Group on Clinical Nursing Education conducted a survey to analyze the components of clinical education in nursing (Ard, Rogers, \& Vinten, 2008). Respondents included 2,218 faculty, representing all types of educational programs, and 28 representatives of state boards of nursing, representing all areas of the county. A summary of the responses to the survey reinforced that clinical education requires the active involvement of a student and teacher and attends to the critical thinking, knowledge, decision-making, understanding, and transfer of knowledge from one situation to another. Findings of the survey supported the value of clinical experiences in providing the foundation for continued development of thinking skills, as well as opportunities for problem solving. Many respondents felt strongly that clinical experiences should be introduced early in the curriculum, occur throughout the program, and involve rotations in all specialty areas. The general consensus of the respondents indicated that clinical brings the classroom and lab to life, is the most valuable component of nursing education, and gives students a sense of confidence to help them pull it all together (Ard, Rogers, \& Vinten, 2008).

Barriers. Educators face many challenges when planning clinical experiences. Results from a 2009 NLN survey of current approaches to clinical education in pre-licensure nursing programs (Ironside \& McNelis, 2010) and the Carnegie study of nursing education (Benner et al., 2010) identified the lack of quality clinical sites as a major barrier to clinical education. As noted in the NLN survey, the number of students enrolled in nursing programs has increased as a result of the nursing shortage. It was further noted that to accommodate the increased number of students, nursing programs have had to compete for clinical sites. However, as a result of shorter hospital stays and unpredictable patient census, in-patient floors available for clinical experiences in acute care hospitals have decreased. This decrease further limits the number of 
clinical sites available for clinical experiences (Ironside \& McNelis, 2010; Tanda \& Denham, 2009; Tanner, 2006a; Waldner \& Olson, 2007).

Lack of qualified faculty, understaffed and overtaxed nursing staff to serve as preceptors for supervision of students, and higher patient acuity that demands closer supervision of students to ensure patient safety have also been cited as barriers to clinical education (Ironside \& McNelis, 2010; Rhodes \& Curran, 2005; Tanner, 2006a). According to the 2009 NLN survey (Ironside \& McNelis, 2010), clinical faculty reported the top three activities that utilized their time during clinical experiences were supervising students ${ }^{\text {ee }}$ skill performance (medication administration, intravenous [IV] therapy, and wound care), assisting students to synthesize clinical information and assessment findings, and questioning students to assess their grasp of their assigned patient"s status. The two most significant challenges reported by clinical faculty in the survey were finding time to provide appropriate guidance and supervision of each student and time to teach students how to think on their feet and make clinical judgments. As reported by Ironside and McNelis (2010), faculty found it difficult to provide individualized student attention to facilitate learning and development of clinical judgment when clinical groups have a high student to faculty ratio. In addition, there is concern for patient safety with the high student to faculty ratios (Benner et al., 2010; Tanda \& Denham; 2009).

Other barriers to clinical education cited in the literature include the lack of standardization of student learning experiences and restrictions imposed by clinical agencies limiting student experiences (Benner et al., 2010; Ironside \& McNelis, 2010; Tanner, 2006a; Waldner \& Olson, 2007). Lack of control over the type of patients and opportunities available for student assignments during clinical experiences and lack of ability to coordinate clinical experiences with classroom content were reported by faculty as challenges to clinical teaching 
(Benner et al., 2010). Additional challenges identified by faculty included the lack of ability to ensure that each student has the opportunity to care for patients with a variety of health conditions and/or to experience critical events. Limitations imposed by clinical agencies, such as calling physicians for orders or administering blood products, also restrict opportunities for student experiences (Li \& Kenwood, 2006). As noted by Li and Kenwood (2006), 44\% of nursing programs do not permit students to call physicians.

A concern for patient safety is always paramount with the traditional model of clinical education (Benner et al., 2010). Students are assigned to care for patients and are expected to intervene quickly when changes occur (Jeffries, 2008). However, few students have the opportunity to actively participate in critical events with real patients. To ensure the safety of patients, it is common for the nurse to take over the care of the patient when the patient takes a turn for the worse or a crisis situation evolves. Ensuring patient safety must always take precedent over student learning opportunities (Decker, Sportsman, Puetz, \& Billings, 2008). Therefore, opportunity to practice the critical thinking and decision-making required for clinical judgments in a safe environment is not provided by this model (Jeffries, 2008). Furthermore, fear of making a mistake can increase anxiety and decrease confidence of students in the clinical setting, increasing the risk for student error and jeopardizing patient safety (Haskvitz \& Koop, 2004).

Strategies to address barriers. The most frequent tactics used to address the barriers of clinical education, identified by 2,386 faculty who responded to the 2009 NLN survey (Ironside \& McNelis, 2010), include:

1. Providing alternatives to the traditional model by using clinical times on evenings, nights, weekends, and/or holidays and substituting clinical hours with simulation activities. 
Faculty who identified that simulation was used as an alternative to clinical experiences reported most often the use of high-fidelity mannequins. Other types of simulation identified, in order of most commonly used, include: role playing, written case studies, medium-fidelity mannequins, video-simulation/case study, virtual or computer-based programs, low-fidelity mannequins, and task trainers. Fifty-six percent of respondents reported substituting $10 \%$ or less of clinical time with simulation, $35 \%$ reported substituting $11-25 \%, 7 \%$ reported $26-40 \%$ of clinical being substituted, and $2 \%$ of respondents reported substituting $41-50 \%$ of clinical time with simulation.

2. Hiring faculty with little or no experience in teaching, supplementing with more part-time faculty, having faculty teach overloads, and partnering with clinical agencies to utilize hospital staff for preceptors.

3. Incorporating more observational experiences, pairing students for patient assignments, and creating other learning experiences on the clinical unit that replaces total patient care.

4. Limiting what students can do (only $50 \%$ give meds), providing more observational experiences to decrease the number of students on the floor, and relying on staff nurses for monitoring and assisting students (Ironside \& McNelis, 2010).

Strategies identified to optimize the student"s clinical education and aid in the development of critical thinking and decision-making skills included the following: creating a safe environment for faculty to question students, making rounds with faculty and staff, using journaling for student reflection on clinical experiences, conducting post-conferences as debriefing sessions for student reflections, and using simulations or case studies that mimic complex clinical situations and require clinical judgments (Ironside \& McNelis, 2010). However, as Ironside and McNelis (2010) note, these tactics and strategies are not without 
problems. Reliance on staff nurses to assist faculty, serve as preceptors, or function as clinical instructors has drawbacks. The priority of staff nurses is patient safety, not student learning. High acuity of patients, in addition to decreases in staffing have resulted in increased workloads of nurses. Furthermore, many staff nurses have no knowledge of the curriculum and little teaching experience. As noted by Brammer (2006), good nurses are not necessarily good educators.

Societal demands for safety and quality, the response of government agencies with the release of the IOM reports, and the professional organizations that regulate safety and quality in health care expect nurse educators to ensure no harm occurs to patients as a result of the educational process (IOM, 2004, NCSBN, 2005). Patients of todaye's healthcare systems are acutely ill with complex needs requiring nursing care involving high-tech equipment (Rhodes \& Curran, 2005). Rhodes and Curran (2005) emphasized that nurses must not only be highly skilled in decision-making, but must also be comfortable and competent with technology. Ethical issues surrounding the traditional model of clinical education, placing students in health care systems to "practice" on patients, are being raised (Kneebone et al., 2004; Reznek, 2004; Ziv, Wolpe, Small, \& Glick, 2003). The education of nurses should never be at the expense of patient safety; patients have the right to expect that they will receive the best care possible without risk of harm (Ziv et al., 2003). Ziv et al. (2003) pointed out that the high acuity of patients and the technology involved in their care can present challenges to novice students and increase risks to patients. However, the authors emphasized that patients have the right to be involved in the decisions surrounding their care and the right to refuse treatment or care by a student. Yet, as Ziv et al. noted, patients may not always be aware they are being treated by a student or aware that a student is performing a skill for the first time. This lack of knowledge 
creates another ethical issue which raised the question by Decker (2007c) "Should students inform the patient if this is their first time at performing a skill?"

A call for change in clinical education. Very little research has been done to guide the design and implementation of clinical education and changes that have occurred have not been a result of strategic planning or rigorous study (Ard et al., 2008, NCSBN, 2005). With the release of the IOM reports, the traditional model of clinical education in nursing is increasingly coming under scrutiny and its effectiveness is being questioned (IOM, 1999; Tagliareni, 2009; Tanner, 2006a). Development of new models of clinical education that optimize student learning and are supported by research has been identified as a need (Ard et al., 2008; Ironside \& McNelis, 2010; IOM, 2001; NCSBN, 2005; Jeffries, 2007; NLN, 2003; Tanda \& Denham; 2009; Tanner, 2006a). The integration of HPS into nursing education, as a supplement to clinical education, is supported by educators, researchers, professional organizations, and regulatory bodies (Del Bueno, 2005; Ironside \& McNelis, 2010; NCSBN, 2006; Nehring \& Lashley, 2010; NLN, 2005b; Smith, 2009; Tanda \& Denham, 2009; Tanner, 2006a; Waldner \& Olson, 2007). Del Bueno (2005) identified the need for exposure of students to consistent practice with real patients as well as visual simulations for the development of effective decision-making and problem solving.

Although advantages are being seen with HPS, there is general consensus in the literature that simulation cannot totally replace traditional clinical experiences; student experiences should include both simulation and actual patients (Gaba, 2004; Katz, Peifer, \& Armstrong, 2010; Laschinger et al., 2008; McGaghie, Issenberg, Petrusa, \& Scalese, 2010; Ziv et al., 2003). Kneebone et al. (2004) proposed that simulation must exist alongside clinical practice to obtain the full benefit of simulation. Berndt (2010) examined the ethical issues of replacing clinical 
experiences with simulation. To experience the significance of caring relationships, values, and preferences; students need human patient contact. Therefore, as Berndt proposed, simulated clinical experiences cannot fully replace clinical experiences.

Diener and Hobbs (2012) raised the question of whether clinical simulation can provide the environment to learn and foster caring behaviors. Can students form and cultivate caring behaviors in their communication and relationship with technology-driven robotic devices? As noted by Diener and Hobbs, caring is the crux of nursing and is a behavior learned primarily through modeling. They further noted that competent care requires not only technological competence, but encompasses humanistic principles of caring within the nurse-patient relationship. For students to form and cultivate caring behaviors, Diener and Hobbs proposed that opportunities must exist early in the nursing program for students to spend time with human beings and witness caring behaviors modeled by instructors.

A unique approach to overcoming the human aspect of patient care lost with the use of HPS was described by Rose, Courey, Ball, Bowler, and Thompson (2012). In efforts to enhance the therapeutic relationship of the nurse and patient, a "therapeutic encounter" was incorporated into simulation. After completing simulated scenarios based on the experiences of an actual human being, students were asked during the debriefing session to identify questions they would have asked the patient if he had been a real patient versus the simulated patient. The following class period, students had opportunity to meet the patient and wife, ask questions, and discuss more personally the physiological and psychological impact of the disease on the patient ${ }^{e e}$ s life and family. Students were overwhelmingly positive about the experience and learned the significance of support systems in the healing process, lifestyle alterations that can occur, and the 
importance of therapeutic communication and trust between the nurse and patient (Rose, Courey, Ball, Bowler, and Thompson, 2012).

Despite the growing use of HPS as a supplement to clinical education, there continues to be a lack of research regarding the amount of clinical experiences that can be safely substituted with simulation (Berndt, 2010; Katz et al., 2010; Ward-Smith, 2008). As noted by Ironside \& McNelis (2010), the extent to which simulation is equivalent to, better than, or worse than learning in actual clinical settings remains to be documented. It is important to differentiate what is crucial for students to learn in a clinical setting and what can be moved to a simulation lab (Jeffries, 2009).

\section{Simulation}

History of simulation in professional education. The field of aviation was the first to introduce the use of simulation in professional education and the earliest flight trainer, developed in 1929 by Edwin Link, became the prototype for the current flight simulators used today (Rosen, 2004). As Rosen (2004) discussed, in attempts to reduce pilot error and improve public safety, flight trainers were developed to provide an easier and safer way to train pilots. By 1955, the Federal Aviation Administration (FAA) incorporated simulation recertification as a requirement for commercial pilots ${ }^{e e}$ license renewal (Rosen, 2004). Nuclear power industries have also used simulation for years to prepare for potential crises (Waxman, 2010).

Simulation in healthcare. In the field of health care, anesthesiology was the first to explore the use of simulation (Bradley, 2006). The first computerized full size HPS (Sim One), developed in the late $1960^{\circ} \mathrm{s}$, was equipped with an anatomically shaped chest that moved with breathing, eyes that blinked, jaws that opened and closed, and pupils that could dilate and constrict (Cooper \& Taqueti, 2004). As noted by Cooper and Taqueti (2004), Sim One was 
designed to train anesthesia residents in the skill of endotracheal intubation. Unfortunately, only one model was built; the mannequin failed to receive acceptance by the medical field due to the cost and the resistance to change from traditional apprentice-based training (Bradley, 2006; Cooper \& Taqueti, 2004).

Harvey, a full-sized mannequin introduced in 1968, simulates 27 cardiac conditions by varying breathing, pulses, normal heart sounds, and blood pressure (Cooper \& Taqueti, 2004). As Cooper and Taqueti (2004) noted, this simulator has been used with success for training and testing bedside cardiovascular examination skills of medical students, interns, and residents. Results of a large research study, sponsored by the National Heart, Lung, and Blood Institute, found that fourth year medical students trained with Harvey performed significantly better than those who interacted only with patients (Cooper \& Taqueti, 2004). In addition, the authors found that students who were able to interpret findings on Harvey demonstrated increased confidence and increased ability to interpret the same findings on patients.

Use of computer-enhanced mannequin simulation did not take off until the late 1980 "s (Reznek, 2004) with the development of two anesthesia simulators which have become the model for today" ${ }^{\text {ec }}$ HPS (Bradley, 2006). Medical simulation experienced rapid growth in the 1990 "s and HPS was being used by many fields in medicine and dentistry by the end of the decade (Rosen, 2004). The use of HPS is also used in the military as a technique to teach trauma and pre-hospital care (Waxman, 2010).

Healthcare professionalse interest in simulation has evolved from the success of use with the military, aviation, and other groups with high risk training. The release of the IOM reports and the need to restructure the educational preparation of health care workers in providing safe clinical care became a major drive behind the use of HPS (Bradley, 2006). As noted by Gaba 
(2004), improvement in the education and training of health care workers serves as the most obvious purpose for the application of simulation in health care education. Acquisition and retention of knowledge can be improved with simulation when compared to the traditional lecture method (Issenberg et al., 1999). HPS provides students with the opportunity to practice communication and clinical skills, participate in teamwork and interprofessional learning, and experience complex clinical situations in a safe and supportive environment (Bradley, 2006). As Bradley (2006) pointed out, the risk of harm to patients is avoided, retention and accuracy of skills increased, and transfer of knowledge from the classroom to practice is improved.

In the medical field, simulation with the use of standardized patients is used for the assessment of performance and competency for licensure (United States Medical Licensing Examination [USMLE], 2011). Rehearsals with simulation can be an adjunct to actual clinical practices such as a surgical team rehearsing an unusually complex operation prior to performing it (Gaba, 2004). Gaba (2004) proposed that the integration of simulation across all disciplines of health care provides opportunity for interdisciplinary teamwork when providing health care for all types and ages of patients. Gaba further suggested that simulation assists with acquiring new knowledge and better understanding of relationships among concepts, increasing confidence with repeated practice in a safe environment, and maximizing learning with instructor feedback.

Research related to HPS in medicine. Research on the effectiveness of HPS in medical and anesthesia education began to surface in the late 1999's. Issenberg, McGaghie, Petrusa, Gordon, and Scalese (2005) conducted a Best Evidence Medical Education (BEME) review spanning 35 years of the features and uses of HPS that led to effective learning. Of the 109 peerreviewed journal articles selected for review, the authors determined that the best evidence supports that, under the right conditions, HPS does improve learning. Ten conditions of HPS 
were identified as being important for effective learning to occur and were summarized in the BEME report (2005) and in a follow up report by Issenberg and Scalese (2007). The need to provide feedback to students regarding their performance was identified as the single most important feature of HPS for effective learning to occur. Other conditions, identified by Issenberg et al. and Issenberg and Scalese, for effective learning to occur with HPS include the following:

- Opportunity for focused repetitive practice that requires students to be actively engaged, as opposed to passive bystanders, decreases the time required for skill acquisition.

- Effectiveness of HPS is best when it is integrated throughout the curriculum in combination with other educational techniques, exposes the learner to a wide range of levels of difficulty progressing from basic skills to higher levels of complexity, and represents a wide variety of standardized patient problems or conditions.

- Clearly defined outcomes for student learning, a controlled environment where students can make mistakes without adverse consequences, and a high degree of realism are important features of HPS.

A lack of quality published research related to medical simulation was identified as a result of the BEME review (Issenberg et al., 2005). As noted in the review, of the 670 articles in the pool for review, only 109 met the inclusion criteria for the review. The need for better and improved medical research on HPS was identified by Issenberg et al. (2005). The authors found many studies with small samples, weak designs, and lack of detail in the methods and data. A follow up review examined simulation-based medical education (SBME) research conducted between 2003 and 2009 (McGaghie, Issenberg, Petrusa, \& Scalesa, 2010). These authors 
determined that the methodological quality and rigor of research during this time period had improved and twelve features and best practices for simulation use were identified. In addition to the features identified in the 2005 BEME review, McGaghie et al. identified the following as best practices for simulation use in medical education: skill acquisition and maintenance, mastery learning, transfer to practice, team training, high-stakes testing, instructor training, and educational and professional context. McGaghie et al. found that simulation was most effective when combined with other educational methods as a complement to clinical education, not as a substitute. The type of SBME used was dependent on the educational goals to be achieved with clinical skill acquisition and maintenance, identified by the authors, as the most common learning objective. Supported by Kneebone et al. (2004), the retention of knowledge and skills is enhanced with regular, repeated, structured practice.

Although results are mixed and more research is needed, studies are showing that some skills (Advanced Cardiac Life Support [ACLS] and management of shoulder dystocia in obstetrics) are retained longer by residents who were trained in a simulation laboratory (McGaghie et al., 2010). Studies are beginning to support that transfer of learning from a simulation laboratory to patient care settings is more effective than learning without simulation. As noted by McGaghie et al. (2010), internal medicine residents trained with simulation, as opposed to those who have not, respond to hospital codes with greater compliance to treatment protocols, have fewer procedural complications with central venous catheter (CVC) insertion, have lower rates of CVC related infections, have improved performance in surgery, and experience fewer complications from shoulder dystocia during birth. These findings were supported by a study that found nurse anesthetists responded more quickly, deviated less frequently from standards, and performed better in crises when trained with HPS 
(Radhakrishnan, Roche, \& Cunningham, 2007). Simulation also provides opportunity for interdisciplinary team training, and as noted by Salas, DiazGranados, Weaver, and King (2008), $70 \%$ of errors in clinical practice result from a lack of communication among the health care team.

Evolution of simulation in nursing education. Simulation use in nursing education has evolved over the last 100 years from using static mannequins for the practice of psychomotor skills to using the advanced human patient simulators of today. In a review of the past 40 years of nursing simulation, Nehring and Lashley (2010) discussed many forms of simulators that have been used in nursing education. As noted by Nehring and Lashley, forms of simulation used by nursing programs have included anatomical models, task trainers, human patient simulators, roleplaying, computer-assisted instruction (CAI), virtual reality and haptic systems, games, and standardized patients. Although advantages and disadvantages have been identified for each type, each has a role in teaching and assessing competency of students (Decker, et al., 2008).

Role-playing has been used for years in nursing education and continues to be a major component of HPS when students assume the role of the nurse (Nehring \& Lashley, 2010). As Nehring and Lashley noted, CAI was first introduced in the late 1970 "s and is growing in popularity and complexity. Nehring and Lashley identified computerized three-dimensional virtual worlds that permit online, interactive, multiuser gaming as opportunities for students to interact and plan nursing care. Decker et al. (2008) pointed out the expense associated with CAI; however, they identified the advantages of flexibility and convenience that CAI provides. As Decker et al. noted, many web-based CAI programs can be accessed from home, be individualized to meet learner needs, provide feedback to the learner, and offer integrated monitoring of the student"s performance for faculty use. 
Standardized patients (SPs), used more in medicine than nursing, provide opportunity for students to communicate, interview, assess, and plan care for a person who has been coached on how to act and role play a particular disease or situation (Nehring \& Lashley, 2010). Cost and the difficulty in recruiting volunteers or paid actors for certain patient populations have been cited as disadvantages by Nehring and Lashley (2010). Standardized patients have been incorporated into the licensing exam of physicians and have shown positive results for validating clinical competencies of nurse practitioner students (Decker et al., 2008).

Virtual reality and haptic systems combine computer technology with task trainers that provide tactile simulation (Decker et al., 2008; Nehring \& Lashley, 2010). As the authors noted, many systems have been developed for surgeons to practice skills, such as scope procedures, and can be used to validate competency. Availability of haptic systems in nursing is limited to a system for IV insertion (Nehring \& Lashley, 2010).

Examples of anatomical models, such as jointed skeletons, and task-trainers, such as mechanical dummies and models of legs and arms were described by Lees (1874) in her book Handbook for Hospital Sisters (Nehring \& Lashley, 2009). Task trainers are equipment or mannequins designed for training of a particular task and can be full body or partial representing a specific anatomical area of the body (Decker et al., 2008). The first full body task trainer can be traced back to 1910 with the birth of "Mrs. Chase," a life-sized mannequin that provided the opportunity for practice of clinical nursing skills taught in the classroom (Herrmann, 1981). Mrs. Chase was first introduced in the classroom in 1911, underwent improvements in functionality in 1914 and 1939 (Nehring \& Lashley, 2009), and was the first mannequin used by schools of nursing in the 1950s (Nehring et al., 2002). In 1913, a baby model was developed, followed by Mr. Chase in the 1940s (Nehring \& Lashley, 2009). These mannequins were static 
and lacked the detail and animation of a real life body and are considered low-fidelity simulators (Seropian et. al., 2004). Their use is most appropriate for learning and demonstrating competency of skills (Decker et al., 2008). Although there is little data that support the use of low-fidelity simulators ${ }^{\text {ee }}$ applicability to clinical practice, their use for introducing and practicing psychomotor skills has been based on the old adage that "practice makes perfect" (Seropian et al., 2004).

In efforts to practice mouth-to-mouth ventilation, Asmund S. Laerdal, innovator and founder of Laerdal Medical AS, created Resusci-Anne (Laerdal, 2012a). As explained in the history of the company, Mr. Laerdal, starting his company in the 1940s, specialized in children $\mathrm{S}$ books and greeting cards before advancing to making toys. With his know-how of producing millions of plastic play dolls, Laerdal created the life-size and life-like Resusci-Anne mannequin for students to learn the lifesaving procedure of resuscitation. It was the belief of Laerdal that students would be better motivated to learn the procedure if it was life-like. The face of ResusciAnne was adopted from the "death mask" of a young girl, pulled from a river in Paris in the late 1890s, who was assumed to have taken her own life. Noted in an article by Cooper and Taqueti (2004), Resusci-Anne, introduced in the $1960^{\circ} \mathrm{s}$, was a low-fidelity mannequin equipped with an internal spring to the chest wall that provided students the opportunity to practice chest compressions. With the development of Resusci-Anne, Laerdal was committed to advancing the cause of emergency care and resuscitation and these mannequins, now available in pediatric and baby size, have become widely used for teaching cardiopulmonary resuscitation [CPR] (Laerdal, 2012a).

As technology progressed, moderate-fidelity human patient simulators, providing more realism than low-fidelity human patient simulators, were developed. These mannequins may 
include heart and lung sounds, pulse and blood pressure, and bowel sounds but lack the corresponding functional eyes or chest movement (Seropian et al., 2004). An example of a moderate fidelity simulator on the market today is Laerdals"s VitalSim product line (Laerdal, 2012c). Described by Laerdal (2012c), when connected to Vital-Sim-enabled mannequins and skills trainers, the VitalSim Vital Signs Simulator can simulate heart sounds, fetal heart sounds, breath sounds, blood pressure and pulses, bowel sounds, and electrocardiographs (ECGs). In addition, these simulators allow for creating, editing, and running preprogrammed and instructor created scenarios.

High-fidelity human patient simulators are full body, life-size, computerized mannequins that have characteristics and physical appearances of patients and are equipped with speaking capabilities, palpable pulses, functional eyes, lung sounds with the corresponding chest wall movement, as well as multiple patient-like functions (Seropian et al., 2004). Fully equipped with computer software, these simulators mimic real physiology that can be programmed to respond to disease states, treatments and interventions, and medications (Pacsi, 2008; Seropian et. al., 2004). High-fidelity simulators provide the most realistic patient situations that allow the student to translate the event into real-life situations (Seropian et. al., 2004).

High-fidelity simulators used in nursing education today are mainly produced by Laerdal and Medical Education Technologies, Inc. [METI] (Nehring \& Lashley, 2004b). The first simulators (Laerdal SimMan ${ }^{\mathrm{R}}$, BabySim, and MetiMan) came fully equipped with a computer, a monitor to display vital signs and physiological parameters, and an air compressor to operate the lungs and physiological functions (Nehring et al., 2002). More advanced models, such as Laerdal"s SimMan 3G and METI"es iStan and HPS, continue to be developed as technology expands. These adult wireless patient simulators have added features that provide more realism 
and may include seizures, simulation of bleeding at multiple sites, secretions, diaphoresis, pupillary responses to light and blinking eyes, skin changes to detect cyanosis, excretion of urine, and/or drug recognition systems with appropriate physiological responses (Laerdal, 2012b; Medical Education Technologies, Inc. [METI], 2012). In addition to adult high-fidelity simulators, pediatric, baby, newborn, and pregnancy simulators are available.

Introduction of HPS in nursing education. HPS was first introduced into nursing education in nurse anesthesia programs and most of the initial research in nursing, appearing in 1998, addressed the use of HPS in nurse anesthesia programs (Nehring \& Lashley, 2004a, 2004b). The use of HPS in pre-licensure nursing programs was first reported to be used in acute care and maternal/child nursing (Nehring \& Lashley, 2004a), but a significant increase in interest and use has been seen the last few years (Katz et al., 2010; Nehring \& Lashley, 2010; Waxman, 2010). In 2002, an international survey of the use of HPS in nursing education was conducted by Nehring and Lashley (2004a). Surveys were sent to the 66 nursing schools that were identified as having purchased a METI HPS. Of the 34 schools that responded, 15 schools offered the associate degree and nine offered the baccalaureate. Nehring and Lashley found that the majority of programs (64\%) acquired their HPS after 1999. In addition, HPS was found to have been used most frequently in associate degree programs to teach physical assessment, technical skills, and advanced medical surgical critical events. Of the schools surveyed, $57.1 \%$ reported that simulation was used as part of clinical time.

Although relatively new to nursing, a study by Katz, Peifer, and Armstrong (2010) demonstrated a growing trend of increased interest in and use of HPS in pre-licensure nursing programs. To assess the use of HPS in baccalaureate nursing programs, Katz et al. sent surveys to $241 \mathrm{BSN}$ programs accredited by the NLN. Of the 78 schools that responded, $78.9 \%$ reported 
using HPS in the core clinical courses of health assessment, fundamentals, pediatrics, medical surgical nursing, and obstetrics. Plans to purchase additional HPS for their programs were reported by $68.8 \%$ of schools. The use of HPS for high-fidelity scenarios was reported by $50 \%$ of the schools, while the other 50\% used HPS for basic assessment, skills and task training. Eighteen schools (23\%) reported replacing actual clinical hours with simulation, but the number of hours varied among the schools, 35\% used HPS for competency testing, and 55\% used HPS to assess critical thinking of their students (Katz et al., 2010).

Both surveys conducted by Nehring and Lashley (2004a) and Katz et al. (2010) identified faculty constraints as one of the major prohibiting factors for the use of HPS. The time required for faculty to learn and become comfortable with the technology and the increased workload associated with developing scenarios, setting up the simulation, and programming the simulator have been identified as barriers affecting the use of HPS (Adamson, 2010; Akhtar-Danesh, Baxter, Valaitis, Stanyon, \& Sproul, 2009; Bray, Schwartz, Weeks, \& Kardong-Edgren, 2009; Howard, Englert, Kameg, \& Perozzi, 2011; Jarzemsky, McCarthy, \& Ellis, 2010; Nehring \& Lashley, 2004a; Radhakrishnan et al., 2007; Starkweather \& Kardong-Edgren, 2008; Tuoriniemi \& Schott-Baer, 2008). Costs associated with the use of HPS are often cited as a major obstacle of implementing simulation (Gordon, Wilkerson, Shaffer, \& Armstrong, 2001; Tuoriniemi \& Schott-Baer, 2008; Ziv et al., 2003). As these authors pointed out, additional costs of space, upkeeps and repairs, and faculty training must be considered in addition to the high cost of the initial purchase of the simulator.

Role of debriefing. As noted by Issenberg, McGaghie, Petrusa, Gordon, and Scalese (2005) and Jeffries (2005), student feedback on his/her performance is essential and one of the most important factors influencing learning. Debriefing, occurring immediately after HPS 
provides the arena for faculty to give feedback and for students to self-reflect on their experience (McLellan, 1996). The debriefing component of simulation is essential to the learning as most of the learning occurs during this time (Alinier et al., 2004; Harder, 2009; Henneman, Cunningham, Roche, \& Cumin, 2007; Jeffries, Clochesy, \& Hovancsek, 2009; Kardong-Edgren, Starkweather, \& Ward, 2008; Kuiper, Heinrich, Matthias, Graham, \& Bell-Kotwall, 2008; Medley \& Horne, 2005; Nehring \& Lashley, 2009; Rauen, 2001; Waxman, 2010).

Debriefing is a focused, facilitated discussion that provides the opportunity to review one ${ }^{e e}$ s performance during a simulated experience (Waxman, 2010). Debriefing provides the environment for the application of knowledge to occur and facilitates the cognitive processes that lead to long-term knowledge (Johnson-Russell \& Bailey, 2010). As Johnson-Russell and Bailey (2010) discussed, students discover meaning of events, relationship between their interventions and patient outcomes, and knowledge for future patient care through guided reflection. As the authors noted, effective debriefing, led by the instructor, can reinforce the objectives of the simulation, clarify information, identify missing links, discuss application to practice, and permit release of emotions surrounding the experience. Johnson-Russell and Bailey also emphasized the importance of providing time for students to explain the rationale for their actions, analyze their actions, and develop alternative interventions to enhance critical thinking and problem solving. Seropian et al. (2004) emphasized the importance of constructive debriefing to avoid the psychological trauma that can be associated with negative feedback and/or negative outcomes of the scenario. As noted by Johnson et al. (1999), a positive, supportive atmosphere throughout the debriefing session facilitates learning.

Critical thinking processes used by senior baccalaureate nursing students, after providing patient care using HPS and guided reflection, were explored in a study by Decker (2007b). As 
defined by Decker, guided reflection is "the process where a facilitator promoted the learner"s development of insight through the use of semi-structured cue questions during the experiential learning experience" (p.45). Decker found that the student"s ability to integrate knowledge, skills competency, and mindset (self-confidence, ability to self-critique, and stress management) influenced the development of critical thinking processes. She further found that critical thinkers demonstrated perceptions of a positive mindset and engagement in reflection-in-action and reflection-on-action. Three themes emerged from Decker"s research as being pivotal to the process of reflection through simulation: specific guidance is needed for the novice to apply experiential knowledge, guidance in the reflective process is required for the learner to progress as a reflective thinker, and the role of the faculty in the development of thoughtful practice is vital to the learner"s success.

Benefits of simulation in nursing education. Advantages of using HPS as an adjunct to clinical are well cited in the literature. Repeated practice of psychomotor skills in a safe environment is vital for learning those skills (Feingold et al., 2004) and repeated exposure to simulation is associated with improved learning outcomes (McGaghie, Issenberg, Petrusa, \& Scalese, 2006). Research shows that opportunities requiring the learner to apply knowledge in multiple situations are more effective in developing higher order thinking (Kardong-Edgren et al., 2008). Learning is facilitated when practice with simulation occurs over a longer period of time with shorter sessions (Feingold et al., 2004) and results in the best outcomes if integrated across the curriculum (Howard et al., 2011; McCausland, Curran, \& Cataldi, 2004; Starkweather \& Kardong-Edgren, 2008). It is suggested that simulation be used early in the program; however, it is important to match course content with simulation content (Henneman \& Cunningham, 2005; Howard et al., 2011). 
HPS offers an important alternative to contextual learning and provides authentic, real world, guided experiences that allow for active student participation in a safe environment without risk to patients (Cioffi, 2001; Feingold et al., 2004; Gaba, 2004; Gordon et al., 2001; Jeffries, 2007; Kneebone et al., 2004; Radhakrishnan et al., 2007; Rauen, 2001). When students experience a critical event with their patients in the clinical setting, traditionally the nurse takes over patient care (Hovancsek, 2007). Although necessary for patient safety, this limits the opportunity for the student to participate in the decision-making or care of a patient during these events (Gaba, 1992). In contrast to clinical with live patients where the instructor is committed to protecting the patient, scenario- based HPS requires the student to independently make decisions about patient care (Howard et al., 2011, Nehring et al., 2002). The student is permitted to make errors in decision-making and patient care without the risk of harm to a live patient (Beyea \& Kobokovich, 2004; Gaba, 1992). Errors provide an excellent opportunity for learning; applying lessons learned from past mistakes to future situations can improve the student"s performance (McCallum, 2007; Ziv et al., 2003). Students can learn to understand and manage clinical situations during times that these situations are replicated with HPS; knowledge learned can later be transferred to a clinical situation when it occurs in real life (Cioffi, 2001; Hovancsek, 2007; Ziv et al., 2003).

The use of simulation in health care is designed to prepare students for situations they may encounter in practice (Harder, 2010). Through a review of the literature by Cant and Cooper (2010), the use of HPS offers the best realism of clinical practice. Scenarios can be designed to provide a wide range of experiences not often encountered with traditional clinical experiences (Jeffries, Clochesy, et al., 2009; Lasater, 2007; Pacsi, 2008; Peteani, 2004; Scherer et al., 2003; Tanda \& Denham, 2009; Waldner \& Olson, 2007) and scenarios of critical health 
incidents that, if occurring in real-life, students can only observe to ensure patient safety (Beyea \& Kobokovich, 2004; Gaba, 2004; Gordon et al., 2001; Howard et al., 2011; Lapkin \& LevettJones, 2011; Li, 2008; Nehring et al., 2001). Student learning experiences can be standardized and HPS provides opportunities for students to practice skills, problem solve, make independent nursing decisions, visualize physiological effects on the body, see the consequences and outcomes of those decisions, and make mistakes in a safe environment without fear of causing harm to patients (Brewer, 2011; Ironside \& McNelis, 2010; Jeffries, 2008; Jeffries, Clochesy, et al., 2009; Kardong-Edgren, Adamson, \& Fitzgerald, 2010; Katz et al., 2010; Lapkin \& LevettJones, 2011; Nehring, 2010; Nehring et al., 2001; Spunt, 2007). Using HPS as an adjunct to acute care clinical assignments can decrease student anxiety (Alinier, Hunt, Gordon, \& Harwood, 2006; Carver \& Marshall, 2010; Irwin, 2011; Jeffries, 2005; McCallum, 2007; Waldner \& Olson, 2007), increase student confidence (Alinier et al., 2004; Bearnson \& Wiker, 2005; Bremner et al., 2006; Cioffi, 2001; Gordon et al., 2001; Haskvitz \& Koop, 2004; Jeffries, 2008; Kuznar, 2007; Nehring et al., 2001; Peteani, 2004; Smith, 2009), facilitate development of critical thinking and decision-making skills (Beyea \& Kobokovich, 2004; Feingold et al., 2004; Gaba, 2004; Gordon et al., 2001; Hayden, 2010; Ironside \& McNelis, 2010; Katz et al., 2010; Medley \& Horne, 2005; Nehring \& Lashley, 2004a; Pacsi, 2008; Rauen, 2001; Rhodes \& Curran, 2005; Smith, 2009; Weis \& Guyton-Simmons, 1998), facilitate application of clinical judgment (Brewer, 2011; Cioffi, 2001; Dillard et al., 2009; Lasater, 2007; Rhodes \& Curran, 2005), improve time management (Smith, 2009), and assist students in applying theory to practice (Beyea \& Kobokovich, 2004; Garrett, 2005; Irwin, 2011; Jeffries, Clochesy, et al., 2009; Kenner \& Pressler, 2011; McCallum, 2007; NCSBN, 2005; Nehring \& Lashley, 2010; Pasci, 2008; Prion, 2008; Rauen, 2001; Spunt, 2007; Weller, 2004). 
The role of simulation in decreasing errors and improving patient care is gaining attention (Gantt \& Webb-Corbett, 2010; Haskvitz \& Koop, 2004; McCallum, 2007; Ward-Smith, 2008). Scenario based HPS provides opportunity for the instructor to identify weaknesses in a student" $\mathrm{s}$ ability to problem solve or perform technical skills (Haskvitz \& Koop, 2004). Exposure to repeated simulation, suggested as a remediation method by Haskvitz and Koop (2004), has been shown to decrease student anxiety, increase confidence, and improve student performance. The students increase in confidence, decrease in anxiety, and opportunity to practice decision-making and problem solving associated with simulation decreases the potential for student error in the clinical area (Haskvitz \& Koop, 2004; Ward-Smith, 2008). In addition to evaluating individual competency, simulation can assist the faculty with identifying gaps and weaknesses in the curriculum, which can be used to guide curricular changes for improved student outcomes (Akhtar-Danesh et al., 2009; Wilson, Shepherd, Kelly, \& Pitzner, 2005). This was identified as one of the benefits of the institution used for this study. After the first semester of simulation, weaknesses within the curriculum and deficiencies in skills became apparent.

Gantt and Webb-Corbett (2010) described the benefits of simulation in teaching patient safety behaviors in undergraduate nursing education. As described by Gantt and Webb-Corbett, patient safety behaviors of hand washing and patient identification were integrated into a checklist for evaluating competency of graduating nursing students. Results of the competency exam revealed that $61 \%$ of students failed to satisfactorily perform hand washing or follow protocol for proper patient identification. Recognizing this as an area needing improvement, changes in the teaching strategies to reinforce these safety behaviors were implemented the following semester. Gantt and Webb-Corbett reported improvement in scores of a follow-up competency exam; $38 \%$ of students failed to perform one or both of the safety behaviors. 
Effective therapeutic communication is essential for providing safe patient care and a key component of nursing education (Kameg, Clochesy, Mitchell, \& Suresky, 2010). Ineffective communication has been identified by the Joint Commission as the most frequent cause of sentinel events (Burke, 2010; Kameg, Mitchell, Clochesy, Howard, \& Suresky, 2009). Nurse educators are challenged with large class sizes and time constraints to provide opportunities for students to practice and adequately evaluate their communication skills (Sleeper \& Thompson, 2008). In addition to providing opportunity for students to practice technical skills, HPS can provide opportunity for students to practice communication skills with patients and other health care professionals (Gaba, 2004; Howard et al., 2011). In two studies, self-reports of student experiences working with HPS to practice therapeutic communication skills, prior to working with psychiatric patients, were positive (Kameg et al., 2010; Sleeper \& Thompson, 2008). Students reported the simulation as helpful in applying theory to practice, alleviating the anxiety related to working with psychiatric patients (Sleeper \& Thompson, 2008), and enhancing student self-efficacy of communication skills (Kameg et al., 2010).

Interprofessional teamwork involving communication and collaboration within and between disciplines has been identified in the IOM reports as being a core educational requirement (IOM, 2003) essential for improvement in patient safety (IOM, 1999) and reduction of patient errors (IOM, 2004). Simulation provides opportunities for students to work in teams as they assess, plan, and implement nursing care (Burke, 2010). As Burke (2010) identified, working together in groups during a simulation promotes teamwork and opportunities for students to see various approaches to problem solving. In addition to promoting teamwork within the discipline of nursing, HPS provides opportunity for students enrolled in multiple disciplines to practice communication and collaboration in the care of a patient (Bray et al., 
2009). During times of a crisis, when a patient ${ }^{\mathrm{ee}} \mathrm{s}$ life depends on the effective interventions and management of the patient"s condition by all members of the health care team, Bray, Schwartz, Weeks, and Kardong-Edgren (2009) emphasized the importance of collaborative teamwork. As identified by Bray et al., simulations involving multidisciplinary health team members are gaining popularity and support of both educators and health care providers. Research on the use of simulation for collaborative interprofessional education is showing positive results (Baker et al., 2008; Reese, Jeffries, \& Engum, 2010).

The goal of nursing education is to ensure that graduates are competent to perform in the role of the professional nurse (NCSBN, 2011). However, learning does not stop when a student graduates. It is imperative that practicing nurses stay abreast of changes in evidence based practice and technological advances to remain competent (Benner et al., 2010). Competency not only requires the acquisition of relevant knowledge and development of psychomotor skills; it also requires the application of knowledge and skills in the practice setting (Decker et al., 2008). As Decker et al. noted (2008), HPS provides a structured environment that can integrate the application of knowledge and skills required in a given context of a patient situation. The use of HPS as a competency assessment for students and practicing nurses is well documented (Decker, Utterback, Thomas, Mitchell, \& Sportsman, 2011; Feingold et al., 2004; Larew et al., 2006; Li, 2008; Nehring \& Lashley, 2009; Prion, 2008). Positive effects are being seen when HPS is used as an adjunct to new graduate orientation (Feingold et al., 2004; Nehring \& Lashley, 2009; Olejniczak, Schmidt, \& Brown, 2010; Shepherd, Kelly, Skene, \& White, 2007), orientation of the novice nurse to critical care (Cato \& Murray, 2010; Rauen, 2004; Stefanski \& Rossler, 2009; Vandrey \& Whitman, 2001), and orientation and continuing education for maternal-child and pediatric nurses (Broussard, Myers, \& Lemoine, 2009; Jeffries, Bambini, Hensel, Moorman, \& 
Washburn, 2009). In addition, use of HPS as another means of competency testing, in addition to the NCLEX-RN, for licensure of new graduates is gaining popularity (Benner et al., 2010; Hovancsek, 2007; Nehring \& Lashley, 2010; Weaver, 2011).

\section{Research on Simulation in Nursing Education}

Review of the literature revealed that research related to simulation use in nursing education has increased exponentially in the last few years. As noted by Harder (2009), the earliest research of simulation in undergraduate nursing education focused on technological issues and accessibility of simulators, how to set up a simulation lab, and teaching and learning strategies for use of simulation. Today, Harder noted that the focus of research is more on evaluating the outcomes of simulation to determine the impact this teaching strategy has on preparing nurses for practice. However, Weaver (2011) identified the need for further research to address the effectiveness of HPS, as compared with traditional clinical, on the development of knowledge acquisition, clinical judgment, and ability to transfer to practice. In this time of scare resources and budget constraints, it is important to determine if the benefits of simulation outweigh the costs (Goodman \& Lamers, 2010; McCausland et al., 2004).

It is important to note the confusion that can surround the terms of simulator, simulation, and HPS found in the literature. Simulation refers to "activities that mimic the reality of a clinical environment and are designed to demonstrate procedures, decision-making, and critical thinking through techniques such as role playing and the use of devices such as interactive videos or mannequins" (Jeffries, 2005, p. 97). The type of physical object or device that is used in the simulated experience, referred to as the simulator, can be standardized patients, partial or full body task trainers, mannequins, and virtual and haptic systems (Cooper \& Taqueti, 2004; Seropian et al., 2004). Full body sized mannequins, referred to as human patient simulators, are 
classified by Seropian et al. (2004) as low-fidelity, medium-fidelity, or high-fidelity. Lowfidelity mannequins, static mannequins that lack detail and realism, have been used for years in nursing education as the standard model for the practice and evaluation of psychomotor skills (Nehring \& Lashley, 2009). The term simulation that is found in the nursing literature today often equates to the use of more advanced technologies such as CAI, virtual environments and haptic systems, and medium- or high-fidelity human patient simulators (Ironside, Jeffries, \& Martin, 2009; Nehring \& Lashley, 2010). Human patient simulation (HPS) refers to simulation experiences that utilize human patient simulators. Today, research evaluating the effectiveness of HPS almost always utilizes a HFHPS; therefore, HPS and HFHPS are often used interchangeably in the literature (Weaver, 2011).

Several reviews of the literature citing the advantages and outcomes achieved with the use of HPS in nursing education have been conducted by nurse educators and researchers (Cant \& Cooper, 2010; Laschinger et al., 2008; Nehring \& Lashley, 2010; Weaver, 2011). Current research is showing evidence that HPS is an effective tool influencing student learning; however, further research is needed to support the outcomes of simulation (Cant \& Cooper, 2010; Harder, 2010; Hyland \& Hawkins, 2009; Nehring \& Lashley, 2010; Pacsi, 2008; Weaver, 2011). In a review of the research on simulation based learning in nursing, Cant and Cooper (2010) noted that the quality of many of the studies on simulation used in nursing education lacked the same rigor as identified by the BEME report. The authors identified that many of the studies on HPS had small and non-representative samples, varied in the designs and methods making it difficult to compare findings, and used assessment instruments with differing levels of reliability and validity. These findings were also identified by Nehring and Lashley (2009) as they noted that many studies exposed both the control and the experimental group to interactive teaching 
techniques that made it difficult to determine results from HPS. Limited exposure of the experimental group to HPS, ranging from one session to a few over the course of one semester, was found in many studies (Cant \& Cooper, 2010). Additionally, Cant and Cooper noted that measurement tools used in some studies were not designed to evaluate HPS. Lack of a formal measurement tool specific for evaluating HPS was identified by Harder, 2010. Further research will require the development of reliable and valid evaluation tools specific to measuring the learning outcomes of HPS (Harder, 2010; Kardong-Edgren et al., 2010). The limitations of the current research on simulation usage in nursing education were also identified by Ironside, Jeffries, and Martin (2009). As these authors identified, the majority of nursing research was conducted in a single course at a single institution, measured faculty and student reaction or response to the simulated experience versus actual outcomes of student performance, and focused on technical skills, problem solving, and implementation of care in response to a critical event.

Lack of hard, experimental data supporting the benefits of HPS are causing some researchers to question if the increased cost and faculty resources of using HPS over low- and medium-fidelity simulation are justified (Gant, 2007; Goodman \& Lamers, 2010; Norman, 2003; Wenk et al., 2009). Results of a study by Wenk et al. (2009) challenged the rationale for using HPS. In a randomized controlled setting, Wenk et al. randomly assigned 33 fourth year medical students to either a HPS or a problem-based discussion (PBD) group of an emergency induction method. Although the HPS group achieved slightly higher scores on a multiple-choice and a clinical skills exam, improvements were statistically insignificant, demonstrating that both HPS and PBD were comparable teaching strategies. An interesting finding of the study was the increased confidence reported by the HPS group on a confidence questionnaire. Students in the 
HPS group overrated their anticipated clinical abilities and knowledge improvement in comparison to their actual performance. This was identified as a negative outcome of HPS and the authors questioned the quality of self-ratings of ability in comparison to externally generated measures of ability as found in most studies.

As a result of the lack of research that supports outcomes of HPS are better than outcomes of low-tech alternatives, Norman (2003) suggested that educators return to low-tech alternatives as a means to save money and allow more students access to this type of training experience. However, Goodman and Lamers (2010) suggested that past studies which reported findings of "no significant differences" with the use of HPS have not asked the right questions. Traditionally, studies compare the "average success" of groups on an identified variable. As Goodman and Lamers pointed out, focusing on average successes may not identify significant changes. As stated by the authors, "The patient is not much interested in the "class average" of nurse-graduates ${ }^{\text {ee }}$ performance as a measure of successful training, or whether simulation can impact that average" (p. 248). Goodman and Lamers suggested using a quality control-type question, "How often per shift or week does someone make an error and does simulation training reduce that risk significantly?" (p.248). Goodman and Lamers proposed that these questions would be more suitable questions to determine the effects of simulation.

As noted by Prion (2008), much of the literature measuring the impact of HPS on nursing educational outcomes has addressed the outcomes of student satisfaction and confidence. These studies, in addition to many studies addressing knowledge and critical thinking, have used selfreports or report-of-others (observational ratings) as data to measure the outcomes of simulation. These types of data are indirect measurements of a variable and have the potential to be unreliable due to possible bias (McGaghie et al., 2010; Prion, 2008). Indirect measurements of 
self-reports or report-of-others should always be combined with other direct measurements to provide a better picture of the research outcomes (Prion, 2008). Direct measurement of learner outcomes, such as multiple-choice questions or simulated patient questions, are more objective (McGaghie et al., 2010), but can be difficult to obtain, and results can be ambiguous with poorly designed methodologies (Prion, 2008). Prion (2008) suggested that direct measurements of student behaviors demonstrating mastery of learning can be determined by the use of test questions given as pre/post tests before and after simulated experiences or use of an objective structured clinical examination (OSCE). Prion further noted the value of using a checklist of identified behaviors that demonstrate knowledge or skill mastery during a simulated experience or videotaping the simulation to be reviewed later to increase the reliability of this tool.

This study examined the outcomes of use of a high-fidelity HPS in comparison to medium-fidelity simulators and the traditional low-fidelity mannequins used for skill practice. Therefore, for the purpose of this study, HPS refers to high-fidelity HPS unless otherwise noted. Focusing on research related to the purpose of the study, the remaining review of literature is limited to research involving the use of HPS in nursing education. The review is organized around the outcomes as identified in Jeffries "simulation model: student satisfaction, confidence, skill performance, knowledge acquisition, and critical thinking. Research addressing the outcomes of knowledge acquisition and critical thinking is discussed in more detail as these are the dependent variables of the study and guided the research questions.

With the concerns and questions of researchers surrounding the value of HPS over lowor medium-fidelity HPS, the fidelity of HPS was one of the independent variables of this study. Research questions were specifically designed to determine how nursing knowledge and critical thinking skills compared among students trained with no HPS, medium-fidelity HPS, and high- 
fidelity HPS. Therefore, research evaluating the effectiveness of low- and medium-fidelity simulation was reviewed and presented separately. This review was beneficial when the results of this study were compared to what is already known about the relationship of the fidelity of simulation and student outcomes.

Low-, medium-, and high-fidelity HPS in nursing education. The review of the literature suggests that overall, simulation with the use of medium-fidelity simulators is perceived by students and faculty as realistic and superior to more traditional methods of instruction for learning and mastery of skills. Students expressed satisfaction with this teaching methodology and reported increased self-efficacy and confidence. It is important to note that most studies were limited to comparing medium-fidelity simulation to traditional methods of instruction such as lecture or case study. Only three studies were found that compared the effectiveness of differences in the fidelity of simulation on student satisfaction and knowledge gains. Results of two of these studies suggest that the use of simulation with a medium- or highfidelity simulator is more effective than the traditional methods of teaching, but the effectiveness of use of a low-, medium-, or high-fidelity simulator on knowledge gain is equivalent.

Kardong-Edgren, Starkweather, and Ward (2008) evaluated student and faculty perceptions of simulation after integrating simulation using a medium-fidelity simulator into a clinical foundations course. Students completed the NLN's Educational Practices, Simulations Design, and Student Satisfaction and Self-confidence in Learning questionnaires after completing each of three scenarios spaced throughout the semester. Student reports of satisfaction and self-confidence in learning remained high with each scenario. Faculty perceptions were mixed, although the opportunity for repetitive practice of foundational skills was perceived to enhance learning by both students and faculty. For the first time, the authors 
reported that all students passed the paper and pencil test at the end of the semester, which was thought to be a result of the additional practice opportunities offered by simulation. Lack of a control group and differences in the level of expertise and comfort of the faculty conducting the simulations were identified by Kardong-Edgren et al. as limitations of the study.

Curtin and Dupuis (2008) reported on students ${ }^{\text {ee }}$ feedback after implementation of a threehour simulation laboratory using a medium-fidelity Laerdal Vital-Sim mannequin. Although the method used to obtain student feedback was not discussed, feedback was positive. Students were satisfied with the learning strategy and reported an increase in confidence. Curtain and Dupuis found that students felt better prepared to identify changes in a patient ec $^{\mathrm{s}}$ condition and reported better performance, compared to other graduates, in a mock emergency during employment orientation.

The use of a medium-fidelity simulator was used to determine the effects of a combined classroom and simulation teaching strategy on students ${ }^{e e}$ self-efficacy and satisfaction (Sinclair \& Ferguson, 2009). A convenience sample of 250 baccalaureate students were enrolled at two sites; one site was the control group while the other site served as the intervention group. Students in the control group attended two-hour lectures on five different lecture topics. The intervention group attended one hour of lecture followed by a simulation for each of the topics. The use of a Likert scale tool, to measure self-efficacy, was given before and after each simulation or lecture, in combination with a student satisfaction survey given after each simulation or lecture. Although self-efficacy ratings improved with each of the five topics in both groups, students in the intervention group demonstrated greater changes in mean selfefficacy ratings. Statistically significant differences were seen in four of the five simulations. Sinclair and Ferguson (2009) found that the intervention group (91\%) was more satisfied with 
the combined lecture and simulation than the control group (70\%) and rated the combined teaching strategy to be more effective (91\%) as opposed to the control group (68\%).

A volunteer sample of 70 nurses participated in a study by Wilson, Shepherd, Kelly, and Pitzner (2005) to assess the realism and suitability of a low-fidelity mannequin, for the purpose of teaching nurses health assessment knowledge and skills. This study used Laerdal ${ }^{\text {ee }}$ Nursing Anne Complete mannequin which was accompanied by a multi-sounds trainer to simulate auscultation of normal and abnormal bowel, breath, and heart sounds. Although this mannequin was classified as a low-fidelity mannequin by the researchers, by definition of this study and most of the literature, this mannequin would be classified as a medium-fidelity mannequin (Seropian et al., 2004). Overall, the results of the study were reported by Wilson et al. as being positive. The authors found that both nurses and nurse educators believed that the majority of the functions and components of the mannequin were realistic for improving clinical performance, superior to existing training products, and suitable for teaching purposes.

As a follow-up to the previous study, Shepherd, Kelly, Skene, and White (2007) examined the effectiveness of using a low-fidelity HPS to enhance graduate nurses ${ }^{\text {ee }}$ health assessment knowledge and skills. Again, the authors identified the mannequin used in the study as low-fidelity (Laerdal Vital-Sim), but by definition of this study and most of the literature, this mannequin would be classified as a medium-fidelity mannequin. The impact of three teaching strategies was compared to determine if one was more effective than another. The sample consisted of 80 newly graduated nurses randomly assigned to one of three groups. After completing five days of formal study, all students were given a pre-test and completed an adult clinical assessment self-directed learning package (SDLP). Group one did not receive any additional training, group two completed two scenario-based PowerPoint workshops, and group 
three completed two simulation experiences with a medium-fidelity mannequin (Vital-Sim). All students were individually tested on performing a systematic patient assessment with a mannequin six weeks after completion of the learning activities. Students were evaluated using a clinical response verification tool of essential actions they were expected to perform. Shepherd et al. found that mean test scores for the simulation group were significantly higher than for the other two groups. Group 1, who received no additional training, performed as well as the scenario-based PowerPoint group. The authors suggested that use of scenario-based simulation could reduce the amount of time required for graduate nurses to become clinically proficient.

Sponsored by Laerdal and the NLN, a national, multi-site, multi-method study to evaluate simulation was conducted over three years (Jeffries \& Rizzolo, 2006). A convenience sample of 403 nursing students enrolled in their first medical-surgical nursing course, representing both BSN and ADN programs from eight schools, was used as the sample. All students completed a 12-item pretest and viewed a videotape of a lecture on postoperative care that included a simulation of caring for a postoperative patient. Students were then randomly assigned to one of three groups who completed either a paper/pencil case study simulation, a hands-on simulation with a low-fidelity static mannequin, or HPS. A post-test was given after the intervention to measure knowledge gain. Jeffries and Rizzolo (2006) reported no significant differences in knowledge gains among the three groups. However, the authors did report that the HPS and lowfidelity mannequin groups reported higher confidence in their ability to care for a postoperative patient. In addition, satisfaction with the learning experience was significantly higher in the HPS group. The paper/pencil case study group perceived fewer opportunities to problem solve; therefore, the authors proposed that this strategy may be less effective in developing these skills. Based on the results of the study, Jeffries and Rizzolo identified active involvement, opportunity 
to apply and synthesize knowledge, and reflective thinking as important components for the development of self-confidence. The degree of simulation fidelity was not identified as affecting the outcomes. In addition, Jeffries and Rizzolo found no significant differences in knowledge gains, satisfaction, or self-confidence with regards to the role the student assumed during the simulation experience. Therefore, the authors proposed that student learning outcomes are not affected by the role assignment of the student during the simulation.

Two studies compared the use of medium-fidelity HPS and HPS to determine if simulation fidelity improved test scores (Kardong-Edgren, Anderson, \& Michaels, 2007; Kardong-Edgren, Lungstrom, \& Bendel, 2009). The first study used a convenience sample of 14 students randomly assigned to three groups: standard lecture only, lecture plus simulation experience with a low-fidelity mannequin, and lecture plus simulation experience with HPS (Kardong-Edgren et al., 2007). A 15 item multiple choice pre-test was given to all participants prior to the lecture and again two weeks later. No statistically significant differences between the pre- and post-tests were found with any group, although there appeared to be more of an improvement in the two simulation groups. The authors recognized the size of the group as a limitation and recommended the study be repeated using larger samples and varying times between pre- and post-tests.

In efforts to compare the costs associated with medium-fidelity simulation as opposed to HPS and the benefits of each on test scores, knowledge retention, and satisfaction; a second study was conducted during a medical-surgical course in a BSN program (Kardong-Edgren et al., 2009). Students from the main campus $(n=100)$ were randomly assigned to one of two experimental simulation groups and students enrolled at two distance campuses $(n=40)$ served as the control. All students took a 15 question multiple-choice exam prior to attending a lecture 
and had access to a study packet and resources on Blackboard. In addition to the lecture, the experimental groups participated in either a simulation experience with a medium-fidelity simulator (Vital-Sim) or a simulation with a high-fidelity simulator (SimMan). The same test given prior to the lecture was given to all students two weeks after the intervention and again at six months. Kardong-Edgren et al. found no significant differences in mean test scores based on simulator fidelity. Although posttest 1 mean scores increased significantly from the pretest in all groups indicating knowledge gain, mean scores of posttest 2 decreased significantly, indicating lack of retention. As noted by the authors, the results of this study did not support that simulation was more effective than lecture for short or long term knowledge gains. In addition, no statistically significant difference in satisfaction, measured by a six-item Likert tool, was found between the two types of simulation. Limitations that might have affected test results were cited by Kardong-Edgren et al. As the authors noted, the simulation experience used for the study was the first simulation experience for the participants and might have affected their ability to learn. In addition, students in the control group formed study groups and increased study time to compensate for the lack of the simulation experience. Kardong-Edgren et al. recommended that the study be repeated with students more experienced with simulation. A quasi-experimental, quantitative study was done to study the relationship between the student"s self-confidence, clinical competence, and simulation (Blum, Borglund, \& Parcells, 2010). Students enrolled in a health assessment and skills course for entry level students served as the participants. Randomly assigned to three laboratory sections meeting once a week for seven hours over the course of the 13-week semester, students used a variety of skills in caring for a patient condition. The control group demonstrated skill proficiency with the traditional low-fidelity task trainers. The other two groups served as the experimental groups who used 
Laerdal ${ }^{\text {ee }}$ S SimMan. Self-confidence measured by student responses to a rubric and clinical competence measured by faculty ratings were completed during mid-term and finals. Blum, Borglund, and Parcells (2010) found that self-confidence and clinical competency for all groups increased significantly from mid-term to final. Although the difference was non-significant, the authors reported that students in the control group using low-fidelity mannequins showed a greater change in self-confidence and clinical competency compared to the experimental group using HPS. Although self-confidence and clinical competency improved regardless of the type of simulation, Blum et al. suggested that the use of low-fidelity simulation may be more appropriate for building fundamental skills and HPS may be more appropriate with advanced students who are more prepared to process complex situations.

Lapkin and Levett-Jones (2011) compared differences in outcomes with a mediumfidelity simulation and HPS to determine if the extra costs associated with HPS was justified. Second $(n=268)$ and third $(n=84)$ year students participated in the study and were assigned to either a medium-fidelity simulation group or an HPS group. Students were evaluated on clinical reasoning, knowledge acquisition, and student satisfaction. Using a checklist of specific behaviors and thinking processes, student performance was evaluated by direct observation of the simulation. A 21-item multiple choice exam, given pre-simulation and two weeks postsimulation, measured knowledge acquisition and a Simulation Experience Scale measured student satisfaction. Lapkin and Levett-Jones found that clinical reasoning scores between the two groups were statistically significant $(p=0.001)$ with the HPS group performing significantly higher on the clinical reasoning checklist. In addition, no statistically significant differences were noted in knowledge acquisition or student satisfaction among the two groups. When the three outcomes were compared with cost estimates, Lapkin and Levett-Jones determined that 
medium-fidelity simulation was the most cost effective approach for achieving the outcomes.

Costs, identified by the authors, required to obtain one unit increase in each of the three outcomes were $\$ 1.21$ for medium-fidelity simulation and $\$ 6.28$ for HPS. As a result, Lapkin and Levett-Jones suggested that effective simulation sessions do not always require HPS, as similar outcomes can be achieved with medium-fidelity simulations.

Nursing research comparing the effectiveness of fidelity on student outcomes is consistent with earlier reviews conducted in medicine. Results have been inconsistent and have not demonstrated that the effectiveness of HPS is superior to medium-fidelity simulation (Goodman \& Lamers, 2010). De Giovanni, Roberts, and Norman (2009) found no significant improvement in a student's ability to identify heart sounds when a HPS (Harvey) was used as opposed to a low-fidelity simulator. As Norman (2003) suggested, one might question the rationale for spending money on HPS if the same results can be obtained using a cheaper medium-fidelity simulator. As the author identified, more research is needed to support that the benefits of HPS outweigh the costs.

Student and faculty perceptions of value and satisfaction. Studies evaluating student and faculty perceptions related to HPS as a teaching strategy reported overall that students value the experience as being beneficial for learning (Abdo \& Ravert, 2006; Bremner et al., 2006; Childs \& Sepples, 2006; Feingold et al., 2004; Howard et al., 2011; Kuznar, 2007; Wotton, Davis, Button, \& Kelton; 2010) and perceive it as realistic (Abdo \& Ravert, 2006; Cantrell, Meakim, \& Cash, 2008; Feingold et al., 2004; Jeffries \& Rizzolo, 2006; Kuznar, 2007). Students appreciated the opportunity to practice cognitive and technical skills in a safe environment and perceived the experience as helpful in understanding content, stimulating critical thinking, and applying theory to practice (Howard et al., 2011, Kuznar, 2007). Although some studies found 
that simulation can increase student anxiety, especially with videotaping, anxiety tended to decrease as exposure increased (Howard et al., 2011). Students appreciated the opportunity to become active participants in a safe environment (Cioffi, 2001). High levels of student satisfaction with teaching strategies have been associated with an increased motivation and interest in learning which can lead to better performance (Kuznar, 2007; Prion, 2008).

A descriptive study was done to measure perceptions of satisfaction of ADN students in their last semester of study after completing HPS in three courses (Kuznar, 2007). Using a 21item Likert-type scale, students were highly satisfied with the use of HPS as a teaching strategy. Kuznar (2007) reported that students rated satisfaction the highest for realism; value of experience for learning patient assessment, development of critical thinking and decision-making skills, and prioritization of skills; and ability to apply theory to practice. These findings were similar to a study by Abdo and Ravert (2006), who evaluated the perceptions of BSN students in the first medical-surgical course after completing five HPSs. Abdo and Ravert reported that students valued the experience and rated the experience as enhancing their learning, developing clinical decision-making, increasing confidence, and improving clinical competence.

Feingold, Calaluce, and Kallen (2004) evaluated faculty and BSN student perceptions after the use of HPS during the senior Advanced Acute Care of the Adult course. After experiencing two HPSs at the beginning and end of the course, students completed a 20 -item satisfaction survey. Feingold et al. found that the majority of students rated the simulated experience as being valuable, realistic, tested decision-making and clinical skills, and enhanced learning. However, the authors found that less than half $(46.9 \%)$ of the students believed the simulated experience increased their confidence or improved their clinical competence (cognitive, affective, and psychomotor skills) and only $54.7 \%$ believed the simulated experience 
prepared them to function in a real clinical environment. One finding of interest pointed out by the authors was the inconsistency between student and faculty perceptions. Although only $50 \%$ of the students believed that learning from HPS would prepare them for practice, $100 \%$ of the faculty believed that the student learning from simulated experiences would transfer to real settings.

After conducting a head-to-toe assessment using a HPS (Laerdal SimMan), novice students"e perception of the experience was positive (Bremner et al., 2006). Of 41 students who evaluated the experience, Bremner et al. found that $95 \%$ rated simulation as good or excellent, $68 \%$ thought simulation should be a mandatory part of their curriculum, and $61 \%$ believed the experience increased their confidence with physical assessment skills. Less than half of the students (42\%) felt this experience relieved stress associated with the first day of clinical. The limited time to work with the simulator was perceived as a limitation by the authors and it was suggested that more exposure might be more effective in reducing anxiety. Although the results supported the use of simulation in undergraduate education, the authors emphasized that the design and integration of the simulation greatly influences what a student learns. Furthermore, Bremner et al. cautioned that simulation must be used appropriately and cannot totally replace clinical with real patients.

Henneman and Cunningham (2005) explored the perceptions of senior nursing studentse experience with HPS in an acute care/critical care elective course. Over the course of the semester, three HPS scenarios with debriefing occurred (one every five weeks). Using a Likerttype tool, faculty and students expressed satisfaction and became increasingly comfortable with this teaching technique. The authors reported that students expressed anxiety over being 
videotaped and the artificial nature of the simulator (skin not changing color); however, these feelings decreased over time.

A study by Childs and Sepples (2006) evaluated students ${ }^{e e}$ response to a simulated laboratory experience. The sample, 55 students enrolled in the senior capstone course, completed a two-hour laboratory session with four learning stations: CD-ROM on cardiac arrhythmias, faculty guided station on identifying rhythm strips, case study designed to identify and plan interventions for arrhythmias, and a mock code with the use of HPS. The HPS station was completed last after exposure to the lecture and the other three laboratory stations. Students identified that the most important features of the HPS were the feedback and information regarding the objectives given prior to the scenario. As Childs and Sepples identified, this study confirmed the importance of student preparation prior to the scenario and debriefing. Although students rated HPS as being very stressful, they rated it very positive and felt they learned the most from this teaching strategy. Comments reported by Childs and Sepples (2006) included "My anxiety was the highest it has ever been" (p. 157); however, many stated they would be less anxious if involved in a code in the clinical setting.

Examining the effects of HPS on student satisfaction and confidence was done in a study by Smith and Roehrs (2009). Mean scores on the satisfaction and confidence scale, after exposure to HPS related to care of a patient in respiratory distress, showed that students were highly satisfied with the teaching method and felt confident in their ability to care for a patient with a respiratory condition. The authors found that clear objectives and a challenging problem to solve were simulation design characteristics significantly correlated with student satisfaction and confidence. A similar study was conducted by Lewis and Ciak (2011). After completing a 
simulation day of four pediatric and four maternal-newborn HPS scenarios, student satisfaction with the learning experience and self-confidence scores were positive.

Limoges (2010) interviewed nine faculty, five BSN students, and four practical nursing students who participated in two to four days of HPS in lieu of traditional hospital-based clinical experiences. The author found that both students and faculty valued the simulation experience. Students identified the simulation lab as the high point in the program with the experience closely matching the actual work of nurses. Comments from students, as reported by Limoges, included "Simulation lab provided me with the opportunity to learn competence and speed when completing skills" (p.60) and "required you to figure out what to do" (p. 61). Faculty described the simulation lab as a way to promote caring and increase confidence.

In efforts to ensure all students had the opportunity to participate in acute episodes, a simulated scenario of an acute exacerbation of heart failure, using SimMan, was incorporated into the first semester senior nursing course of a BSN program (McCausland et al., 2004). After attending a lecture on heart failure, reading materials and written objectives were given to the student. Conducted during a clinical post-conference, 72 students participated in and evaluated the simulation. Overall, McCausland et al. reported that $90 \%$ of the students rated the experience positive, with over half rating it as excellent. Additional findings reported by the authors included: $82 \%$ of the students reported they had come to the simulation prepared to make decisions, $83 \%$ reported pre-scenario reference materials were helpful, $96 \%$ believed the experience and debriefing were helpful to their learning, and 97\% reported simulation would help them in future situations. As a result of the positive evaluations, the authors recommended that HPS is incorporated across all levels of the curriculum. 
Articles are abundant that support the value of using HPS in undergraduate nursing programs. Students consistently rate the experience as valuable and are very satisfied when HPS is used as a teaching strategy. Student and faculty satisfaction are important to the success of simulation (Jeffries, 2007). When students are satisfied with a learning experience, they are more apt to be motivated and interested, which can enhance the learning experience (Kuznar, 2007). Faculty satisfaction is imperative for faculty buy in. If the faculty are not satisfied with a teaching strategy or do not value it as beneficial to learning, faculty will not commit the time and energy required to learn the technique (Akhtar-Danesh et al., 2009).

Self-efficacy, self-confidence, and competency. It is not uncommon for students to experience anxiety and fear associated with harming a patient or making a mistake in the clinical setting (Benner et al., 2010). Most frequently, this fear is correlated with the degree of selfconfidence and perceived competence of the student (Lundberg, 2008). Confidence in the ability to perform well affects a nursing student ${ }^{\text {ee }}$ s competence in performance and is essential for successful nursing practice (Leigh, 2008). As Leigh (2008) noted, confidence permits students to shift their focus from their own needs and focus on the needs of the patient. Self-efficacy, defined by Leigh as the belief in one $e^{e e}$ abilities to carry out a task or accomplish something, is often used interchangeably and reported as confidence, defined as judgment of one se $^{\text {seception }}$ of ability. Furthermore, Leigh noted that confidence increases as self-efficacy increases.

Self-confidence, more importantly the belief in one $e^{\text {ee }}$ ability, provides the motivation to invest in learning (Lundberg, 2008). Lack of confidence can interfere with a student's ability to acquire new knowledge. As acknowledged by Lundberg (2008), if students do not believe they will be successful, they will be less willing to attempt the task. Lundberg further noted that repeated successes have been identified as the most effective way to develop self-confidence. 
Therefore, Lundberg urged nurse educators to foster and nurture confidence in students to facilitate the acquisition of knowledge.

Self-confidence and feelings of competency are identified as two important factors influencing clinical decision-making (Hagbaghery et al., 2004; White, 2003). A study by Brown \& Chronister (2009) revealed that higher critical thinking scores were significantly related to ratings of higher self-confidence. Studies have shown that self-efficacy and confidence of graduate nurses are directly related to improved patient care, easier transition into practice, job satisfaction, and job retention (Leigh, 2008).

Several studies have looked at the effects of HPS on the outcomes of students"e selfefficacy and confidence and findings were mixed. A review of the literature on HPS and students" self-efficacy was conducted by Leigh (2008) and students"e perception of confidence and competence with the use of HPS was reviewed by Yuan, Williams, and Fang (2012). In the review by Leigh, several articles were identified as showing an increase in students"e self-efficacy after participating in HPS. Repetition and repeated practice with realistic clinical situations with HPS can increase a student"s confidence and competency (Blum et al., 2010). Confidence increases as students become familiar with clinical situations (Johnson et al., 1999). Generally, students reported decreased levels of stress and increased confidence working with patients in the clinical setting after having experience with HPS (Leigh, 2008).

In the study by Jeffries and Rizzolo (2006), students perceived experiences with HPS as a positive event that increased confidence. This was supported by research conducted by Abdo and Ravert (2006), Kuznar, 2007, Lasater (2007) and Rystedt \& Lindstrom (2001). A qualitative descriptive study of 50 practicing nurses was conducted by Gordon and Buckley (2009) to determine the degree of improvement in confidence after exposure to HPS. All participants 
reported an increase in confidence with performing technical and nontechnical skills. Students identified the debriefing as the most valuable component of HPS. These results were consistent with a study by Schoening, Sittner, and Todd (2006) that used a convenience sample of 60 BSN students to examine students "erceptions of HPS. Schoening et al. found that students were satisfied with their ability to achieve the stated learning outcomes with the experience, valued the hands-on learning for skill proficiency and opportunities to use decision-making skills, and reported an increased sense of confidence and self-efficacy in how to act as a nurse.

Using a convenience sample of 112 BSN students, Bambini, Washburn, and Perkins (2009) evaluated students"e perception of self-efficacy after being exposed to a three-hour postpartum simulation lab in preparation for clinical. Using a pretest-posttest design, Bambini et al. found a significant increase in the students ${ }^{\text {ee }}$ perception of self-efficacy and confidence after participating in the simulation. Qualitative comments indicated that students learned the importance of communicating with patients and significant others, gained confidence in psychomotor skills, and developed clinical judgment.

A study exploring the effects of multiple HPS experiences on students ${ }^{\text {ee }}$ perceived confidence and competence was conducted by Mould, White and Gallagher (2011). Students enrolled in a critical care nursing course were exposed to 27 scenarios over the course of the semester (three scenarios per week for nine weeks). Students actively participated in 17 or 18 of the scenarios and observed the others. Mould et al. found that both confidence and competence scores, from a self-report survey completed by the students, improved significantly from the beginning to end of the course. In addition, the authors found that $65 \%$ of students reported they appreciated and enjoyed the experience. It was further noted that those who stated they enjoyed and appreciated the experience reported higher levels of confidence and competence. This study 
supported earlier studies that found experience, through a series of multiple scenarios, has a positive effect on improving confidence and competence. Furthermore, results of the study support the need for nurse educators to ensure that learning strategies are fun and perceived as having value by students.

A pilot study to evaluate the implementation of a respiratory distress HPS scenario was conducted by Garrett, MacPhee, and Jackson (2010). Thirty seniors volunteered to participate and focused groups were used to collect data. Garrett et al. found that students valued real-time patient status changes with minimal faculty intervention throughout simulation. The opportunity to see what would happen to a patient based on their actions during the scenario was also identified as being very beneficial. All students reported feeling more confident after having the chance to practice and observe real-time change. Furthermore, the authors found that students least appreciated the teamwork component of simulation, stating they would rather work alone or in pairs. Some students expressed feeling awkward and anxious with videotaping, but students reported these feelings decreased over time.

An exploratory descriptive study by Bearnson and Wiker (2005) examined the advantages and limitations of using high-fidelity HPS in lieu of clinical. Two groups of first year BSN students participated in a two-hour HPS session with three post-operative scenarios in place of a clinical day. Students reported that experiences with medication administration in the simulated environment directly let to increased knowledge, ability, and self-confidence.

In contrast, two studies by Alinier and Hunt $(2004,2006)$ that compared HPS with the traditional lecture showed a gain in knowledge with the use of HPS, but no differences were noted with confidence. These findings were consistent with the study by Feingold et al. (2004); Scherer, Bruce, and Runkawatt (2007); and Blum, Borglund, and Parcells (2010). Results of the 
study by Feingold et al. showed that less than half of the students exposed to HPS believed the experience increased confidence or improved clinical competency. The study by Blum et al., comparing the effectiveness of skill training on low-fidelity simulation and HPS, revealed that self-confidence scores improved significantly from pretest scores to posttest scores. Although no significant differences in the improvement of self-confidence were noted between the two groups, Blum et al. did point out that the group trained with low-fidelity simulators demonstrated a greater change in self-confidence when compared to the HPS group. The study by Scherer et al. had similar findings. Comparing the effectiveness of instruction with HPS and case study, the case study group scored significantly higher on posttest confidence.

The inconsistencies found with studies examining the effectiveness of HPS on students ${ }^{\text {ee }}$ confidence level warrants further research in this area. Many of the studies on self-efficacy and confidence were qualitative with small samples and results generated from open-ended questions or anecdotal notes (Leigh, 2008; Yuan et al., 2012). Comparing self-efficacy and confidence before and after a simulation experience has been a component of many research articles (Shinnick, Woo, \& Mentes, 2011). However, Shinnick, Woo, and Mentes (2011) pointed out that the measurement tools most often used to measure self-efficacy and confidence were researcher developed Likert-type scales with varying ranges and many studies did not report validity and reliability of the tool. In addition, research is lacking that supports if confidence gained in a controlled safe environment is actually transferred to the clinical setting (Weaver, 2011; Yuan et al., 2012). It was also noted that more research is needed to explore the relationship of self-efficacy on knowledge gain and skill improvement (Shinnick et al., 2011) and clinical performance and patient safety (Leigh, 2008). 
Skill performance. Psychomotor skills are an integral part of nursing content taught in the classroom. The use of simulation for practicing skills can be traced back to the earliest use of simulation in nursing education (Nehring \& Lashley, 2009). As Nehring and Lashley (2009) identified, partial and full body task trainers, such as Mrs. Chase, were designed to provide opportunity for students to become proficient in skills prior to performing on a live patient.

It is well known that use of task trainers for practicing skills facilitates skill competency (Issenberg et al., 2005). However, repetitive practice, with static mannequins and task trainers, does not provide the opportunity for students to learn to perform those skills in the context of a nurse-patient relationship (Yoo \& Yoo, 2003). As Yoo and Yoo (2003) noted, incorporation of psychomotor skills into HPS provides opportunity for students to perform skills as they interact with a patient or learn to adapt the skills as required for individual patient situations.

In a study by Jeffries, Woolf, and Linde (2003), use of an interactive CD-ROM was equally effective in teaching the skill of performing an EKG as the traditional lecture, demonstration, and hands-on practice. Although statistically significant gains in knowledge and skill acquisition were found between pretest and posttest scores of both groups, Jeffries et al. found no significant differences between the groups.

Yoo and Yoo (2003) compared the effectiveness of standardized patients as a method for teaching fundamental skills. The control group, students enrolled in the nursing fundamentals course, received the traditional method of lecture, demonstration, and practice on static mannequins in the laboratory for learning basic skills. The following semester, students enrolled in the course were instructed on the same skills by demonstration and practice on a standardized patient (SP) with the exception of the invasive skills that were practiced on mannequins. Using SPs plus mannequins for demonstration of invasive skills, all students were evaluated at the end 
of the semester on their performance of skills. Yoo and Yoo found that students in the SP group scored significantly higher in clinical judgment (identification of relevant data, patient problems, and skills necessary for the case), clinical skill performance, and communication skills than those in the traditional group.

HPS training was compared to problem-based learning for teaching critical assessment and management skills (Steadman et al., 2006). Thirty-one fourth year medical students were randomly assigned to two groups, one receiving instruction by a problem based case study presentation and the other by a scenario using HPS. Steadman et al. (2006) found that mean posttest scores compared to pretest scores were significantly improved for critical assessment and management skills in the HPS group. Inconsistent with the findings discussed earlier by Wenk et al. (2009), results of this study suggest that use of HPS is superior to PBL.

Two studies by Alinier, Hunt, and Gordon (2004) and Alinier, Hunt, Gordon, and Harwood (2006) compared the effectiveness of HPS and traditional lecture on nursing students ${ }^{e e}$ clinical skills and competence. The first study (2004) examined the effects of adding a human patient simulator session to the traditional teaching methods. All participants, during the middle of their fourth semester, tested using an Objective Structured Clinical Examination (OSCE) of 15 stations to determine the initial baseline measurement of students ${ }^{\text {ee }}$ dinical and psychomotor skills. Students were then randomly assigned to a control group receiving the traditional curriculum or the experimental group receiving two simulation sessions at the beginning of the fifth semester in addition to the traditional curriculum. A questionnaire, to collect information on confidence level and stress, was completed prior to a second OSCE testing the middle of the fifth semester. Although both groups improved their scores on the second OSCE, Alinier et al. 
(2004) found that students who participated with HPS performed significantly better $(\mathrm{p}<0.05)$.

The authors found no significant differences in confidence levels between the two groups.

In a follow-up study, Alinier et al. (2006) examined the effects of scenario based HPS on nursing students ${ }^{\text {ee }}$ clinical skills and competence. A sample of 99 second year students was randomly assigned to a control group who received the traditional instruction and clinical experiences or the experimental group who received six hours of HPS in addition to the traditional instruction and clinical experiences. A pretest OSCE, given to all students prior to the instruction, was compared to a posttest given six months after the instruction. Alinier et al. found that the experimental group had a significantly higher improvement in performance on the OSCE as compared to the control group. No differences in perceptions of stress or confidence were noted among the two groups. These findings indicate that HPS was no more effective in decreasing stress and increasing confidence than the traditional lecture and clinical experiences. However, the authors identified a correlation between stress and confidence; students who reported a lack of confidence also reported feeling stressed.

A purposeful sample of students enrolled in their final semester at eight schools of nursing within the Indiana University system, representing both BSN and ADN education, was exposed to two HPS experiences caring for four patients (Ironside, Jeffries, \& Martin, 2009). The first simulation was conducted during week three or four, with the second occurring between weeks nine or ten. The purpose of the study was to determine if experiences in multiple patient simulations improved students ${ }^{\text {ee }}$ achievement and implementation of patient safety competencies. An investigator-developed tool, designed to measure 16 KSA criteria from the Quality and Safety in Education in Nursing project (QSEN), was used to determine if the KSA criteria were demonstrated during the simulation. Students demonstrated significant improvement in patient 
safety performance during the second simulation, suggesting that immersion in HPS with multiple patients increases the achievement and implementation of patient safety competencies.

The primary causes of medication errors are inexperience and distractions (Sears, Goldsworthy, \& Goodman, 2010). To assess the effectiveness of the use of simulation-based instruction for increasing safety with medication administration by new nurses, a randomized control study of BSN students, enrolled in a medical, surgical, and maternal child clinical course, was conducted. Students $(\mathrm{N}=54)$ were randomly assigned to the treatment or control group. The treatment group was exposed to simulated case scenarios in place of some clinical time in the first seven months of the course. The introduction of medication administration in the clinical setting was started week seven for both the treatment and control group. Results reported by Sears et al. indicated that the control group had a greater number of medication errors as opposed to the treatment group. These results suggest that prior exposure to a related simulation experience results in fewer medication errors.

Radhakrishnan, Roche, and Cunningham (2007) examined the relationship of HPS on students"e clinical performance of safety, basic assessment skills, problem-focused assessment skills, appropriate interventions, communication, and delegation. Using a quasi-experimental pilot study, 12 senior BSN students were randomly assigned to the control or intervention group. The intervention group participated in two (one hour practices), evenly spaced throughout the semester, of caring for two patients with complex diagnoses using HPS. The control group had no practice with HPS throughout the semester. Both groups were evaluated at the end of the semester with HPS involving a two patient assignment. Utilizing a clinical simulation evaluation tool (CSET) to identify expected behaviors, Radhakrishnan et al. found that students who practiced with HPS achieved significantly higher scores for basic assessment skills (assessing 
and monitoring basic vital signs) and safety (patient identification) than the control group. Other sub-scores were reported as being comparable between the two groups. The study by Shepherd et al. (2007) found similar results. Mean test scores of a systematic patient assessment were significantly higher for the group who received additional training with scenario-based HPS, as opposed to the group receiving additional scenario-based PowerPoint workshops and the control group with no additional training.

The available research supports that HPS is effective in developing and enhancing both basic and advanced clinical skills. Incorporation of psychomotor skills into HPS provides opportunity for students to practice skills within the context of a patient situation. However, studies evaluating the use of interactive CD-ROMS, SPs, and low- to medium-fidelity simulators have revealed similar results as HPS. As educators, it is important to determine what teaching methodologies are most effective for obtaining the established learner outcomes. In times of scare resources, a low-fidelity simulator may be just as effective in developing clinical skills as the more expensive HPS. Evaluating the cost-effectiveness of HPS for teaching undergraduate nursing students has been identified as an area for future research (Lapkin, Levett-Jones, Bellchambers, \& Fernandez, 2010).

Knowledge. Acquisition of nursing knowledge is essential for safe clinical practice. Nursing is a practice art that requires the application, analysis, and synthesis of content for decision-making and clinical judgments in clinical situations (Rauen, 2001). According to Bloom "s taxonomy; the application, analysis, and synthesis of knowledge require first, an understanding of content (Forehand, 2005). Nurse educators are responsible for designing curricula and teaching strategies to ensure their graduates have the knowledge and skill necessary for safe practice (Benner et al., 2010). Three literature reviews (Cant \& Cooper, 2010; 
Lapkin et al., 2010; Shinnick et al., 2011) provided evidence that exposure to HPS can influence knowledge acquisition. However, findings are mixed and some studies have shown no gain or a decrease in knowledge following simulation.

Several studies have used student surveys to measure the students ${ }^{\text {ee }}$ perception of understanding of classroom content with HPS. In a study by Hunter and Ravert (2010), students reported an increased understanding of course material after participating in HPS scenarios. Similar findings were found by Comer (2005) and Bearnson and Wiker (2005). After participating in a simulation using role-playing, Comer found that students reported an increased understanding of course content and faculty noticed a decreased failure rate on the course exam. After substituting one day of clinical for a two-hour lab involving three HPS scenarios, Bearnson \& Wiker (2005) found that students reported an increase in knowledge, skill, and confidence in medication administration. Self-assessments of students were obtained in a study by Dillard et al. (2009) to examine students ${ }^{\text {ee }}$ perceptions of the effectiveness of using HPS to teach heart failure. Dillard et al. reported that all students indicated they believed they understood or mostly understood all of the six learning objectives. These findings suggest that classroom material can be reinforced with simulation.

Although limited, there are studies that have used an objective assessment tool, such as a test or a competency performance exam, to measure the effectiveness of HPS on the acquisition of knowledge. Lewis and Ciak (2011) investigated the impact of HPS on student satisfaction, confidence, cognitive level, and critical thinking. Prior to a simulation day of four pediatric and four maternal-newborn HPS scenarios, students were given a 20-item multiple-choice pretest to measure baseline cognitive level. After completing the simulation day, a posttest identical to the pretest was given to measure gains in cognitive level. Lewis and Ciak found a significant gain in 
knowledge with posttest scores. However, no control group was used to determine if the same results would have occurred with use of other teaching strategies.

Similar findings were found by Burns, O'Donnell, and Artman (2010) who tested the effectiveness of using HPS to develop students ${ }^{\text {ec }}$ knowledge and understanding of the nursing process. A convenience sample of students enrolled in the Introduction to Professional Nursing course received a two-hour lecture on the nursing process, completed a ten-item multiple choice exam on the content one week later, and then participated in a three-hour HPS of 12 evolving patient scenarios. The scenarios were designed for students to have the opportunity to observe and experience the complexity of applying the nursing process. Each student actively participated in one of the scenarios while observing the others. A 10-item multiple-choice exam was given one week after the HPS laboratory as a posttest to measure knowledge gain. Burns et al. found a significant gain in knowledge $(\mathrm{p}<.001)$ in posttest scores, supporting the value of adding HPS to the traditional lecture delivery. Again, no control group was used. To measure attitude changes, a 14-item attitude instrument was given pre- and post- simulation. Significant improvements in attitude, identified by Burns et al., were demonstrated on six of the 14 items: overall nursing knowledge, critical thinking skills, specific skills for patient care, confidence in nursing skills, communication with patients, and communication with other team members.

In a study by Elfrink, Kirkpatrick, Nininger, and Schubert (2010) that evaluated the impact of a simulated experience on knowledge, similar findings were revealed. Students from two courses, second year medical surgical course $(n=41)$ and third year high acuity course $(n=43)$, volunteered for the study. Prior to the HPS and immediately after, students were given a two question written pretest to measure pre- and post-simulation knowledge of the content. Questions relating to the simulation content were included on the final exam. Overall, Elfrink et 
al. found significant improvements between the pretest and posttest scores for both the second and third year students. However, the authors noted differences in the retention of knowledge from post-simulation to the final exam. Of the second year students who answered the questions correctly on the posttest, $93 \%$ retained the information and answered correctly on the final. On the other hand, Elfrink et al. reported that the third year students were less successful, with only $50 \%$ of students correctly answering the questions on the final. The study did support that students can learn from HPS, however, the lack of retention of content in the high acuity course did raise a concern and the authors identified a need to explore other educational interventions for greater knowledge retention.

Brannan, White, and Bezanson (2008) compared the effectiveness of instruction on cognitive skills and confidence with the traditional lecture and HPS. Using a quasiexperimental, pretest/posttest design, students enrolled in the adult health nursing course fall semester served as the control group and received a two-hour lecture on a content area. The intervention group was comprised of students enrolled in the course spring semester. This group participated in a two-hour lab, culminating in HPS, in lieu of a lecture. The intervention group received significantly higher posttest scores than the control group on a written test measuring cognitive skills. Although both groups demonstrated a gain in confidence, the differences were not significant. These findings suggest that learning can occur and be equivalent with both lecture and HPS.

The use of HPS to teach critical care nursing in a senior level undergraduate nursing program was evaluated to determine the effects on basic knowledge (Hoffmann, ODonnell, \& Kim, 2007). Using a pre- and posttest repeated measure design, 29 students enrolled in a seniorlevel medical surgical nursing course served as the participants. All students completed 45 hours 
of didactic content followed by 45 hours of traditional clinical on a medical surgical or stepdown from intensive care unit. The last 45 hours were spent in the simulation lab and each student participated in at least four HPS scenarios. The assessment tool used for measuring knowledge was the Basic Knowledge Assessment Tool-6 (BKAT-6), a 100-item test measuring application of basic knowledge required for critical care. The test was given the first day of class and again at the completion of the course. Hoffmann, O'Donnell, and Kim (2007) found significant improvements in the overall scores and six sub-scores of the posttest. The two sub-scores that did not show a significant difference were two areas not addressed in the HPS scenarios. No control group was used to compare traditional clinical with HPS. All students were exposed to lecture, traditional clinical, and simulation, so it is difficult to determine the direct effect of simulation on the knowledge gain. The authors cited this as a limitation of the study.

Scherer, Bruce, and Runkawatt (2007) used a pre- and posttest quasi-experimental design to evaluate the effectiveness of HFS and case study seminar on knowledge and confidence. All 23 nurse practitioner students completed a pretest after receiving a PowerPoint presentation on atrial arrhythmias. Randomly assigned to two groups, the experimental group participated in a scenario using HPS and the control group completed a one-hour case study seminar with open discussion among the class. The same scenario on atrial arrhythmia was used by both groups. Although Scherer et al. found improvements in posttest knowledge scores for both groups, differences were not significant. These findings suggest that the addition of HPS or case study did not improve knowledge learned from the didactic presentation. Although posttest confidence scores improved in both groups, scores of the control group were somewhat higher. The authors speculated that this might have been a result of the design of the study. The control group had opportunity to discuss and problem solve the management of the patient with input from the 
whole group, as opposed to the experimental group who completed the HPS individually. These findings were similar to the study by Jeffries and Rizzolo (2006), who found that the effectiveness of HPS on knowledge gain was no different than the effectiveness of a case study or low-fidelity simulation.

In response to the lack of quality clinical sites, many nursing programs are utilizing HPS to supplement traditional clinical hours (Ironside \& McNelis, 2010). Although limited, research comparing the outcomes of HPS and the traditional model of clinical education is beginning to surface. The NCSBN research initiative completed a pilot study to compare the effectiveness of HPS to actual clinical experiences (Hicks, Coke, \& Li, 2009). Senior BSN students (n=58) enrolled in a critical care course participated in the study. Three outcomes were measured: knowledge, clinical performance, and confidence. Knowledge acquisition was measured by a 50-item written exam reflecting course content, self-confidence was measured by a 12 -item Likert-type self-confidence scale, and clinical performance was measured by student performance during three scenarios with standardized patients. After all students completed the three credit hour didactic portion of the course, a 50-item written test and self-confidence scale were given to measure baseline knowledge and confidence. Randomized to one of three practicum groups, students participated in either 30 hours of simulation without clinical experiences, 15 hours of simulation and 15 hours of clinical experiences with a critical care nurse preceptor, or 30 hours of clinical experiences with a critical care nurse preceptor. A posttest written exam, self-confidence scale, and clinical performance exam were given at the completion of the practicum. Interestingly, Hicks, Coke, and Li (2009) found that all three groups had significantly lower posttest scores compared to pretest scores. The students averaged a retention rate of $86.3 \%$ of the didactic content after the practicum experience. The clinical group retained 
the most $(88.5 \%)$, while the simulation group retained the least $(82.9 \%)$. No significant differences were found between the groups on the clinical performance exam, although the faculty tended to rate higher the students in the combination and clinical group. Approximately one-third of the students in each group were rated as not performing at a satisfactory level. The authors further found that students in the combination and simulation groups had statistically significant gains in self-confidence levels after the practicum experience, while no significant change was noted for the clinical group. Although limitations were cited (size of group, previous clinical and simulation experiences of the student, and validity of the confidence scale to actually measure confidence or attitudes), the study does add support to the literature that HPS may be equivalent to traditional clinical experiences in achieving the outcome of knowledge acquisition, clinical performance, and self-confidence.

A study examining the differences between HPS and traditional clinical experiences on the development of critical care nursing skills and critical thinking used a quasi-experimental posttest comparison of two sample groups (Beddingfield, Davis, Gilmore, \& Jenkins (2011). Students enrolled in the final semester of an associate degree nursing program $(n=24)$ participated in the study. After attending class related to the objectives of critical care nursing, the group was divided, with one group attending a traditional clinical lab in a critical care unit of a hospital and the other group attending a scenario with the use of HPS to care for a postoperative patient with a dysrhythmia. The groups were then switched to provide care for a patient on a ventilator with a pressure alarm emergency using the opposite instructional method. Questions related to the care of these two types of patients were included on a critical care examination. Beddingfield et al. found no statistically significant correlations between test items and the type of clinical experiences. Although limitations were identified by the authors, the 
small sample size and the lack of proven reliability and validity of test items, the findings suggest that HPS may be a valid alternative to traditional clinical teaching. However, further research to support this is needed.

In attempts to evaluate if HPS clinical experiences are equivalent to traditional clinical in teaching basic nursing care in a fundamentals course, a study was conducted in a BSN program (Schlairet \& Pollock, 2010). After completing a knowledge pretest, students were randomly assigned to either traditional clinical experiences in a nursing home or HPS clinical experiences in the simulation laboratory for two weeks. After completion of the experience, a knowledge posttest was given and students were then switched to spend two weeks in the other type of clinical experience. Posttest 2 was given to all participants at the end of these two weeks. Schlairet and Pollock (2010) found that significant gains in knowledge occurred from pretest and posttest 1 and from posttest 1 and posttest 2 for both traditional and simulation experiences. Knowledge scores of the traditional clinical group were statistically equivalent to the group exposed to simulated clinical experiences regardless of the sequencing of the two experiences. These findings suggest that HPS clinical experiences are equivalent to traditional clinical experiences regarding the acquisition of knowledge. Schlairet and Pollock did identify that the group who was exposed to simulation first had a steeper positive incline from pretest to posttest 1 than the group exposed to traditional clinical first. These findings support the research of others who suggest that simulation experiences can best be used early in a program to prepare students for traditional clinical experiences. Limited sample size and the short intervention phase of four weeks were cited as limitations. However, this study was a first to look at the effects of sequencing and timing of HPS and traditional clinical. Further research is needed to determine optimal ratios and sequencing of traditional to simulated clinical experiences. 
Kuiper, Heinrich, Matthias, Graham, and Bell-Kotwall (2008) explored the impact of HPS on situated cognition and how this teaching strategy compares to traditional clinical experiences in undergraduate nursing students. A purposeful sample of 44 students enrolled in an adult health medical surgical course completed Outcome Present State-Test (OPT) worksheets after clinical experiences in the hospital setting and after completion of HPS. As described by the authors, the OPT is a model of clinical reasoning that is designed to structure cognition, encourage reflection, and improve clinical judgments of students. Scores of OPT worksheets completed by students after traditional clinical were compared to those completed after HPS. Kuiper et al. found no significant differences between the mean scores of the two groups. However, the authors found that the OPT scores for the HPS group were higher in the area of connecting present-outcome states and NANDA diagnoses, recording laboratory data, making judgments regarding tests, and listing interventions. Findings suggest that HPS offers a reliable opportunity for students to practice clinical reasoning skills essential for nursing practice.

As noted by many of the reviews, most of the studies evaluating the effectiveness of HPS vary on the amount of exposure students have to HPS. Studies ranged from one single exposure to multiple exposures occurring in one day or spaced throughout one or two courses or semesters. A study by Sportsman, Schumacker, and Hamilton (2011) evaluated the impact of replacing traditional clinical experiences with scenario-based HPS in a regional simulation center (RSC) across the curriculum of a BSN and ADN program. The percentage of traditional clinical time substituted with HPS was identified in the following courses: health assessment (100\%), fundamentals (50\%), medical-surgical (25\% each), childbearing (8\%), pediatrics $(13 \%)$, and capstone (20\%). No time was spent in the RSC for psychiatric nursing or the BSN community course. Over the course of the three-year study, 895 students participated in one of six cohorts. 
Differences in exposure to HPS occurred between the cohort groups and were dependent upon when the cohort entered the program during the three-year period. Exposure to HPS varied from having no experience to having up to five semesters. Graduating GPA and scores on a standardized exit examination were used to measure the impact of HPS on the three cohorts of graduating seniors during the study. Sportsman et al. (2011) found no significant differences in the means of GPA upon graduation and exit examination scores for the cohorts of seniors graduating in the three-year period. As the authors noted, the amount of exposure to HPS and percentage of time HPS was replaced with traditional clinical experiences did not have a negative effect on the students"exit examination scores. This study is one of the first to evaluate the impact of HPS when integrated across the curriculum of a nursing program and examine the impact of HPS when substituted for traditional clinical experiences. Findings of this study show promise of the value of HPS as a viable substitution for traditional clinical and continuing research is needed for further support.

In addition to measuring knowledge gain immediately after HPS, studies are evaluating the effectiveness of HPS on knowledge retention. Although studies demonstrate that knowledge acquisition occurred after HPS, it was not always retained. Spring 2000, Nehring et al., (2001) used a convenience sample of 42 senior nursing students in an advanced medical-surgical course to validate knowledge retention. Students were given a pretest after being exposed to lectures on four content topics. Students were then exposed to case scenarios of these events using the HPS. Posttests were given at the completion of the scenarios and five to seven days later. Nehring et al found that significant differences $(\mathrm{p}<0.5)$ existed between the pretest and posttest but no differences were found between the two posttests. Results demonstrated that learning and shortterm retention of knowledge occurred with the use of HPS. 
In the study by Kardong-Edgren, Lungstrom, and Bendel (2009), posttest scores showed a significant increase in knowledge gain after simulation with both medium-fidelity and HPS. However, knowledge was not retained when scores of a second posttest, given six months later, decreased significantly. Similar results were found by Bruce et al. (2009). Graduate and undergraduate nursing students participated in a mock cardiac arrest HPS. The purpose was to evaluate the effects of HPS on knowledge, confidence, and clinical competence of graduate nurses in managing a cardiac arrest. Undergraduate students were evaluated on knowledge acquisition only. After receiving instructions specific to the educational level, graduate nurses were pretested on their knowledge and confidence of managing a cardiac arrest and undergraduate students were pretested on their knowledge. After participation in the HPS, both graduate nurses and undergraduate students were again tested with the same pretest. After participating in two HPSs, the graduate nurse"s ability to manage the arrest was evaluated by a Student Competency Scale. Bruce et al. found a significant gain in knowledge between pretest and posttest scores of graduate nurses. Although confidence scores and competency of skill performance increased, differences were not statistically significant. Undergraduates were tested for knowledge both immediately after the HPS and four to eight weeks later. Bruce et al. found significant differences in knowledge gain between scores on the pretest and posttest 1 . No significant differences were found between the pretest and posttest 2 mean scores, suggesting knowledge retained was similar to pre-simulation knowledge. However, the authors found a statistically significant decline in scores between posttest 1 and 2, suggesting a lack of retention of knowledge. No control group was used to compare results and this was cited as a limitation of the study. 
Ackerman (2009) examined the effectiveness of adding a cardiac arrest scenario using HPS to the standard American Heart Association"s (AHA) Cardiopulmonary Resuscitation (CPR) adult course. The purpose of the study was to evaluate differences in the acquisition and retention of knowledge and skills between students taught with the standard course and those taught with HPS in addition to the standard course. The sample of 49 students completed a pretest to measure current CPR knowledge since all students had received the AHA CPR training previously. After participating in the standard CPR review course, students were randomly assigned to the control group and the experimental group. The first posttest of CPR knowledge, measured by a multiple-choice exam of questions from the AHA exam, and CPR skill, measured by a demonstration of CPR in a mock code, was given after the experimental group participated in the HPS. Retention of CPR knowledge and skills was evaluated with the same tools three months later. Ackerman found that both the control and experimental groups had significant gains in knowledge from pre- to posttest. The experimental group demonstrated significantly higher scores on the multiple-choice exam and the CPR skills exam compared to the control group. These same findings were found on the second posttest given three months later. However, the authors noted that both the control and the experimental group showed a significant decrease in knowledge and CPR skills on the second posttest. These findings were consistent with previous findings indicating a loss of retention of content. Although findings from this study indicated lack of retention of knowledge and skills after six months, the addition of a simulated experience to the standard course had a positive effect and significantly increased CPR knowledge and skills at the time of the course and three months later.

Research objectively evaluating the effects of HPS on knowledge acquisition remains sparse and elusive (Shinnick et al., 2011). The need for further research, especially in the area of 
the effectiveness of HPS clinical experiences in comparison to traditional clinical experiences, will help both educators and professional regulatory bodies determine the best mix and sequence of these two experiences. A few studies have suggested that knowledge learned in simulation is transferred to the clinical setting. However, these reports have mainly used the students ${ }^{\text {ce }}$ and faculty ${ }^{\text {ees }}$ perceptions of knowledge transfer. More research using objective measurements of knowledge transfer as opposed to subjective data has been identified as a need.

Although results of the studies evaluating the impact of HPS on knowledge retention were mixed, it is important to note that the study indicating that knowledge was retained retested the students five to seven days after the intervention. Students were retested at much longer intervals in the other three studies that demonstrated a lack of knowledge retention. In these studies, the time frame for retesting varied from one to six months. These studies did identify the need for repetition and reinforcement of the student"s learning and Bruce et al. (2009) suggested that exposure to repetitive experiences with HPS may be needed for the etention of knowledge. The need for more research in this area was identified.

Critical thinking. Clinical reasoning and accurate clinical judgment are essential components of nursing practice (NLN 2000, 2010; AACN, 2008). Effective clinical reasoning

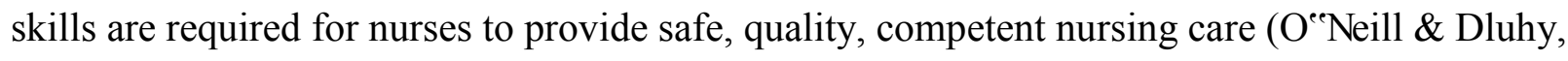
1997). Development of critical thinking skills is essential for effective clinical reasoning and clinical judgment (Alfaro-LeFevre, 1995; Facione \& Facione, 2008). Facilitating the development of critical thinking is a frequently cited advantage of HPS. Simulation provides opportunity for students to make decisions independently without causing harm to a patient. Studies have examined the impact of HPS on critical thinking and findings are elusive (Shinnick et al., 2011). As Shinnick et al. (2011) noted, few studies exist that have had ample sample size 
and objectively measured critical thinking. Many studies have relied on self-reports of student"s perceptions of their ability to use critical thinking for clinical reasoning and clinical judgment (Cant \& Cooper, 2010).

Several studies used various methods to subjectively evaluate student"s and faculty"s perceptions of the impact of HPS on critical thinking abilities of students. A descriptive, quantitative method was used to evaluate undergraduate BSN students"e perceptions of simulation across the curriculum (Hunter \& Ravert, 2010). Participants included 162 students enrolled winter semester 2010 in the second through fifth semester of the program. After completing HPS activities integrated into each course, students completed a survey to identify the learning outcomes they perceived to have improved or developed through the simulation experience. Hunter \& Ravert (2010) found that the most beneficial outcomes of simulation, reported by students, were an increase in nursing skills and development of critical thinking skills.

Confidence was identified as the third; however, the authors noted differences among the groups of students. Hunter and Ravert found that fundamental nursing students identified an increase in nursing skills as the greatest benefit of simulation, whereas students in the remaining courses identified development of critical thinking. The authors suggested that this finding supported the importance of the student developing confidence in skill development before he/she can focus on other skills such as critical thinking. It was interesting to note that facilitating teamwork, as a beneficial outcome of HPS, was the only outcome that was ranked higher by students each semester. Hunter and Ravert suggested that teamwork is developed as the student progresses through the program.

Student and faculty responses to the integration of the use of HPS as a new teaching pedagogy were reported by four studies (Guhde, 2010; Horan, 2009; Lasater, 2007; Rhodes \& 
Curran, 2005). Integration of a HPS scenario into four semesters of an associate degree program was received with positive results (Horan, 2009). Of the 57 students responding to the survey, Horan (2009) found that students reported the use of HPS as being effective in helping them to understand the didactic concepts (98\%), feel more capable to care for patients $(88 \%)$, enhance confidence (89\%), and make more clinical decisions (89\%).

Students ${ }^{\text {ee }}$ responses were positive to an on-line exercise and HPS scenario incorporated into a medical surgical course in the junior year of a BSN program (Guhde, 2010). A complex clinical situation was developed and presented as a case study for students to discuss on-line. After completion of the case study, each student participated in HPS to care for the patient, debrief, and self-reflect on his/her own thinking. On a 5-point Likert scale, Guhde (2010) found that students rated the assignment effective in utilizing critical thinking skills (4.7) and enhancing awareness of the importance of patient assessment (4.8).

Similar findings were revealed in a study by Lasater (2007), who utilized a focus group to collect data regarding students"e perception of the weekly integration of HPS into the junior level Nursing Care of the Acutely Ill Adult course. Students"e comments, reported by Lasater, pointed to the effectiveness of HPS in bridging the gap between the classroom theory, skills laboratory, and clinical practice; in providing a wide array of experiences; in making one think and anticipate what might happen, and in fostering collaboration. As one student stated, "she appreciated others" perspectives and approaches because they made her more flexible in her thinking, giving her a broader range of interventions, based on clinical judgment, to apply to the same patient care issue" (Lasater, 2007, p. 274). Students identified the lack of the ability of the mannequin to engage in nonverbal communication and reveal certain abilities such as reflexes 
and skin color changes as limitations of HPS. Most of the participants discussed a desire for more direct, honest feedback regarding the severity of their actions on patient outcomes.

In a pilot study of 21 senior medical surgical students, Rhodes and Curran (2005) surveyed students to obtain feedback regarding their perception of the effectiveness of HPS experiences in enhancing critical thinking skills. Rhodes and Curran found that student responses were positive, noting the experience was beneficial in requiring the use of critical thinking skills. The faculty reported that the experience provided opportunity for students to engage in critical thinking and problem solving. In addition, the ability to observe students using these thinking processes during the simulation was perceived by faculty to be beneficial.

Lisko and O`Dell (2010) provided opportunities for students to practice simulated critical thinking opportunities in laboratory experiences throughout a 15 -week medical surgical nursing course. A scenario based skills evaluation the last week of the course, which included skills and theory learned throughout the semester, received positive comments from faculty and students. Lisko and $\mathrm{O}^{e e}$ Dell found that students viewed the experience as helpful in bringing together knowledge from the classroom, skills learned in the laboratory, and clinical experiences. As the authors noted, the experiences required students to think independently and intervene, thus facilitating critical thinking and increasing confidence.

Fero et al. (2010) utilized a quasi-experimental design to compare student performance and critical thinking skills between groups of students randomly assigned to video-taped vignette (VTV) or HPS in the last semester of their nursing program. Participants included diploma students $(n=14)$, associate degree $(n=12)$, and baccalaureate degree students $(n=10)$. The California Critical Thinking Disposition Inventory (CCTDI) and the California Critical Thinking Skills Test (CCTST) were used to measure critical thinking dispositions and skills. A researcher 
developed assessment tool was used to measure knowledge and performance in six categories: recognizing the problem, reporting data, initiating interventions, anticipating medical orders, providing rationale for decisions, and prioritizing care. Students were randomly assigned to either the VTV or HPS group after completing the CCTDI and CCTST. After orientation and practice, students were administered either the VTV or HPS testing scenario. Students were then alternated and after orientation and practice were tested for performance with the alternative strategy. Fero et al. found no significant differences in performance between the VTV or HPS group regardless of the order of the intervention. The authors further found no statistically significant relationship between critical thinking dispositions and skills and overall VTV performance. However, a statistically significant relationship was found between HPS performance and CCTDI and a negligible relationship was found between HPS performance and CCTST scores. Students with strong critical thinking dispositions performed higher on the HPS. The authors suggested that teaching and evaluation using HPS may support the development of critical thinking skills and performance outcomes.

A similar study by Ravert (2008) examined differences in critical thinking between three groups of students: control group ( $\mathrm{n}=15)$, small group discussions $(\mathrm{n}=13)$, and HPS group $(\mathrm{n}=12)$. All three groups participated in the regular instruction. One experimental group participated in additional one hour small group scenario-based discussions weekly for five weeks and one group participated in five one hour weekly simulations for five weeks. Using a pretest/posttest design, critical thinking dispositions and skills were measured with the CCTDI and CCTST. Ravert noted a moderate effect size in CCTDI with the two experimental groups and a large effect size for the control group, although differences between groups were not significant. The CCTST scores revealed a large effect size for the two experimental groups and 
a moderate effect size for the control group with no significant differences between groups.

Ravert noted that critical thinking dispositions are ingrained traits and more difficult to change, which may have contributed to the lower gains in CCTDI. Small sample sizes and the ability of the measurement tools to measure content specific to nursing were cited by the author as limitations.

A comparative study measuring the effectiveness of HPS on outcomes of critical thinking utilized 140 senior BSN students enrolled in an ECG course (Brown \& Chronister, 2009). The control group received 100 minutes of the lecture format teaching weekly (400 hours total), while the experimental group received 70 minutes of lecture and 30 minutes of simulation with HPS weekly (500 hours total). Elsevier"s Evolve ECG custom exam; a 30-item, multiple-choice, computerized exam written at the application level or higher; was used to measure critical thinking. Subcategory scores were reported for assessment skills, therapeutic nursing interventions, and critical thinking. Brown and Chronister (2009) found no significant differences in critical thinking scores or sub-scores between the groups.

Critical thinking and knowledge. Two unpublished dissertations examined the effectiveness of HPS on the development of critical thinking skills and knowledge. Schumacher (2004) used a descriptive, quasi-experimental, pretest-posttest research design to compare critical thinking abilities and learning outcomes utilizing three instructional strategies: classroom, HPS, and a combination of both. All study participants, undergraduate baccalaureate students, completed a 60 -item customized HESI exam designed to measure the cognitive level of content prior to the intervention. Randomly assigned to one of the three instructional strategies, students participated in three learning activities to care fo a patient experiencing a critical event (myocardial infarction, pulmonary embolism, and anaphylactic/hypovolemic shock). A 20-item 
HESI exam was given at the completion of each of the learning activities to measure critical thinking and learning outcomes. Schumacher found no significant differences in critical thinking abilities or learning outcomes with the group exposed to classroom instruction. Significant differences in learning outcomes and critical thinking abilities were noted with the groups exposed to HPS or a combination of HPS and classroom instruction.

A multi-site, quantitative, quasi-experimental pretest-posttest design was used to compare the effectiveness of HPS and interactive case study (ICS) on knowledge gain and critical thinking abilities (Howard, 2007). A convenience sample of 49 nursing graduates from two nursing programs (diploma and BSN) were randomly assigned to one of two groups after completing a customized HESI exam measuring the content of ICS and HPS. After participating in two scenarios (acute coronary syndrome and cerebral vascular accident [CVA]) by either ICS or HPS, students were given a posttest with a different customized HESI exam using the same test blueprint as the pretest. HESI pre- and posttest scores were used to measure knowledge gain and critical thinking abilities. Significant differences were found by Howard in both knowledge gain and critical thinking abilities with the HPS group. Students ${ }^{\text {ee }}$ perception of the experience with regards to value, stimulation of critical thinking, transferability to clinical, decreasing anxiety in the clinical setting, and understanding of concepts were more positive with the HPS group.

Although many studies support that students and faculty perceive HPS as an opportunity to develop critical thinking skills, few studies have used objective quantitative tools to support this. Of those studies, findings are mixed. No significant differences were found on studentse critical thinking skills when HPS was compared to small group discussions or on students ${ }^{\text {ee }}$ performance when HPS was compared to VTV. One study did support that HPS was more 
effective in increasing critical thinking and knowledge when compared to interactive case studies. Findings of three studies that compared HPS to traditional lecture were inconsistent with one study showing HPS was more effective than lecture, while the other two demonstrated no significant differences. The need for further research that supports the impact of HPS on the development of critical thinking skills is needed.

\section{Summary}

Nurses, adequately prepared with the knowledge, clinical reasoning, and clinical judgment to practice in today"s health care systems, are essential for patient safety (Jeffries, 2007). Research documents that the majority of entry level nurses are not prepared to practice effectively in today"es health care systems (Del Bueno, 2005) and many leave their jobs in the first year or two of practice (Pellico et al., 2009) . Educational programs, preparing graduates to practice as professional nurses, are being called upon to make changes to better prepare graduates for the complexity of today"s health care systems (Durham \& Alden, 2008).

The effectiveness of the current model of education in providing adequate opportunities for student learning, which relies on the apprenticeship model of placing students in a clinical setting to care for real patients, is being questioned. The increased complexity and acuity of patients in hospital settings, as well as the technology involved in patient care, are not conducive to learning for the inexperienced, beginning student (Jeffries, 2007). In addition to lack of sufficient quality clinical sites, opportunities for standardization of student experiences and ensuring students have exposure to a wide variety of complex patient conditions and critical events without risk to patient safety have resulted in a gap between education and practice. As a result, graduates are experiencing difficulty transitioning from the role of student nurse to professional nurse. 
Educators have a moral responsibility to ensure that students are provided with the best learning experiences available to maximize learning and prepare them for practice (Ziv, et al., 2003). Opportunities to apply knowledge and develop critical thinking skills, essential for clinical reasoning and judgment, must be sufficient and effective to achieve the outcomes required for professional nursing. An abundance of research supports the benefits to learning when the student is actively involved in the learning and is provided with opportunities for the application of knowledge and practice of critical thinking and decision-making. Supplementing lecture content with HPS, interactive case studies, and PBL can move students from the level of understanding of knowledge to the level of analysis, synthesis, and evaluation.

HPS is showing great promise as an innovative teaching pedagogy to assist with delivery of classroom content, overcome the limitations imposed by the traditional clinical model, and to supplement or replace traditional clinical experiences. The benefits of using HPS for clinical experiences are rooted in experiential and situated learning theories. Hands-on learning, active participation, and opportunity for reflection, all provided by simulation, are conducive to learning. HPS provides students with experiences to care for patients within the context of the real world that are not always provided with clinical experiences. The ability to practice within a safe environment, without risk of harm to patients, is frequently cited as the primary advantage of HPS. Opportunities to practice critical thinking and decision-making, communication, technical skills, and application of knowledge in a risk free environment have been shown to increase student confidence, decrease risk of error in the clinical setting, and facilitate the transfer of knowledge to practice. The use of HPS in undergraduate nursing programs is growing in popularity as a means of preparing the novice student nurse for patient care, assisting 
students in the application of theory to practice, and transitioning the graduate from the student role to the professional role of practice.

The review of literature revealed that there are numerous articles published relating to the use of HPS in nursing education and the number continues to grow. However, there is a paucity of research evaluating the impact of HPS on student outcomes. A multitude of studies are available that have evaluated student and faculty perceptions of the effectiveness of HPS. These studies have demonstrated that students enjoy, value, and are satisfied with HPS as an instructional strategy for effective learning. However, these studies have relied on subjective self-reports of the student ${ }^{\text {ee }}$ s and/or faculty ${ }^{e e}$ s perceptions of confidence, competence, knowledge gain, and critical thinking skills. The reliability of self-perceived ratings as valid indicators of actual learned skills raises questions. Research that provides hard core evidence of the impact of HPS on the student outcomes of knowledge acquisition, development of critical thinking skills, and increased confidence and competency is limited and many studies lack ample sample size. Further studies, using objective assessments with reliable and valid instruments to measure the impact of HPS on these student outcomes, are needed.

The use of HPS as a complement or alternative to traditional clinical experiences is gaining attention as a way to overcome the limitations of these experiences and ensure patient safety. Educators are struggling to find the appropriate balance of HPS and traditional clinical experiences that result in the best student outcomes. Studies evaluating the impact of simulation, when used as an alternative to traditional clinical experiences, are limited and findings have been inconsistent. In addition, the majority of studies evaluating the impact of HPS with a valid and reliable objective assessment tool limited the HPS exposure and varied the sequencing and timing of the placement of the exposure. HPS exposure ranged from one experience to four or 
five that occurred over the course of one day, a few weeks, or a semester and varied in the placement within the curriculum.

Research evaluating the outcomes of HPS integrated throughout an undergraduate curriculum is very limited. One study evaluated the sequencing of HPS and traditional clinical to determine if there were differences in outcomes depending upon when the student was first exposed to HPS or traditional clinical in the first semester of the program. Only one study was found that used an objective assessment tool to measure the outcome of knowledge acquisition when HPS was used as a substitute for a percentage of traditional clinical across the curriculum. No studies were found that used an objective assessment tool to measure the outcome of critical thinking when HPS was integrated throughout a nursing curriculum. Research shows that repetitive practice and time are required for development of critical thinking skills. Studies that expose students to more experiences with HPS over a longer period of time will provide more evidence of the impact of HPS on student outcomes of knowledge acquisition and critical thinking.

As a result of the literature review, it is clear there is a need for continuing nursing research to examine if the use of HPS improves student outcomes or patient care. Research that supports the positive outcomes of HPS is needed to justify the cost and increased faculty time and resources associated with HPS. Studies that explore the appropriate combination and sequencing of HPS and traditional clinical will provide educators and regulatory bodies with sound nursing evidence to guide curriculum decisions and improve educational outcomes.

This study examined the impact on knowledge acquisition and critical thinking after HPS was integrated across the curriculum of an associate degree program. In addition, the impact of medium-fidelity and HPS on these outcomes was compared. Results from this study contributes 
to the research currently available to educators as they continue to determine the best uses and methods for incorporating HPS into the curricula of undergraduate nursing programs. Finding the best mix of HPS and traditional clinical that results in the highest level of student performance will better prepare students to function in today ${ }^{\text {ee }}$ s health care systems. Our patients, who have entrusted us with their care, will ultimately benefit the greatest as nurses are better prepared to provide the quality care they have the right to expect. 


\section{Chapter 3: Methodology}

This study focused on the effectiveness of human patient simulation (HPS), when used as an educational teaching strategy in nursing education, by examining how the use of HPS impacts the critical thinking and knowledge of graduates of an associate degree nursing program. The relationship between HPS, critical thinking abilities, and knowledge was explored to determine if differences existed in nursing students ${ }^{\text {ee }}$ critical thinking abilities and nursing knowledge in relationship to the fidelity of the HPS and the amount of HPS in which the student participated. More specifically, the impact on student outcomes of critical thinking skills and nursing knowledge was examined in relation to the percentage of traditional clinical experiences substituted with HPS. This study was not a direct cause and effect study. It determined only if there was a correlation between any significant change in critical thinking scores and knowledge in relation to the type of fidelity and amount of student exposure to HPS.

\section{Participants}

The population for this study was graduating nursing students from the associate degree nursing program at a public community college in West Virginia. The study was confined to one institution to control for the following variables:

1. Students admitted to the program were admitted using the same admission selection criteria throughout the time span of the study.

2. The curriculum of the nursing program has remained consistent throughout the study.

3. Although there was some faculty turnover in the classroom and clinical setting throughout the time span of the study, the faculty member conducting the HPS learning experiences remained consistent throughout the study. 
4. Other than the incorporation of HPS into the curriculum, educational strategies for the classroom, campus lab, and clinical education have remained fairly constant throughout the time frame of the study.

Sample. The sample for this study was a convenience sample drawn from the students admitted to the nursing program August 2004 through August 2008 and graduating May 2006 through May 2010. Students who withdrew from the program and/or repeated courses were excluded to control for equal exposure to nursing content, clinical experiences, and HPS within each cohort. The final sample $(\mathrm{N}=187)$ represented all graduates of May, 2006 through May, 2010 who were successful in completing the nursing program in the prescribed two years. Post hoc power analysis was done to determine if sample sizes were adequate.

The sample was further divided into six cohorts by date of graduation, with each cohort being exposed to different amounts and levels of fidelity of HPS. The May 2006 graduating cohort, exposed only to low-fidelity mannequins for skill acquisition, had no exposure to HPS and served as the control group. The other five cohorts were exposed to varying amounts of either medium-fidelity or high-fidelity HPS.

Demographics. Demographic data describing the sample by cohort are depicted in Table 1. Of the 187 graduates, 176 were female and 11 were male. Males were represented with each cohort and ranged from one to three per cohort. Three cohorts were all Caucasian; the other three had one graduate of Asian or Hispanic descent per cohort.

Descriptive statistics describing the demographic characteristics of gender, ethnicity, and age by cohort are found in Table 2. As depicted in the table, cohorts were similar in regards to gender and ethnicity, with all cohorts predominately female (94.1 percent) and Caucasian (98.9 percent). The mean age of the six cohorts ranged from 24.93 years to 28.33 years. 
Table 1 Demographic Data of Sample by Cohort

\begin{tabular}{|c|c|c|c|c|c|c|c|c|}
\hline \multirow{2}{*}{$\begin{array}{c}\text { Admission } \\
\text { Date }\end{array}$} & \multirow{2}{*}{$\begin{array}{l}\text { Graduation } \\
\text { Date }\end{array}$} & \multirow{2}{*}{$\begin{array}{l}\text { Cohort } \\
\text { Number }\end{array}$} & \multicolumn{2}{|c|}{ Gender } & \multicolumn{2}{|c|}{ Ethnicity } & \multicolumn{2}{|c|}{ Age } \\
\hline & & & $\bar{F}$ & $\bar{M}$ & White & non-White & $\mathrm{T}$ & $\overline{\mathrm{NT}}$ \\
\hline $8 / 2004$ & 5/2006 (G1) & 33 & 32 & 1 & 32 & 1 & 16 & 17 \\
\hline $8 / 2005$ & 5/2007 (G2) & 39 & 36 & 3 & 39 & 0 & 20 & 19 \\
\hline $8 / 2006$ & $5 / 2008(\mathrm{G} 3)$ & 45 & 42 & 3 & 45 & 0 & 29 & 16 \\
\hline $8 / 2007$ & 5/2009 (G4) & 22 & 20 & 2 & 21 & 1 & 14 & 8 \\
\hline $1 / 2008$ & $12 / 2009$ (G5) & 22 & 21 & 1 & 21 & 1 & 14 & 8 \\
\hline $8 / 2008$ & 5/2010 (G6) & 26 & 25 & 1 & 26 & 0 & 17 & 9 \\
\hline
\end{tabular}

Note. G1 - G6 = group $1-$ group $6, \mathrm{~T}=$ traditional students (ages 18-24), NT = nontraditional students (ages 25 and older)

Table 2 Descriptive Statistics for Demographics by Cohorts

\begin{tabular}{lccccccc}
\hline Cohort & N & \multicolumn{2}{c}{ Female } & \multicolumn{2}{c}{ Caucasian } & \multicolumn{2}{c}{ Age } \\
\hline & & Frequency & Percentage & Frequency & Percentage & Mean & SD \\
\hline 1 & 33 & 32 & 96.97 & 32 & 96.97 & 28.33 & 9.46 \\
2 & 39 & 36 & 92.31 & 39 & 100 & 26.23 & 7.56 \\
3 & 45 & 42 & 93.33 & 45 & 100 & 24.93 & 7.83 \\
4 & 22 & 20 & 90.91 & 21 & 95.45 & 25.95 & 9.3 \\
5 & 22 & 21 & 95.45 & 21 & 95.45 & 25 & 7.12 \\
6 & 26 & 25 & 96.15 & 26 & 100 & 25.77 & 7.45 \\
\hline
\end{tabular}

Academic abilities on admission. To determine if cohorts were equal in regards to academic abilities and critical thinking skills on admission to program, descriptive statistics were performed on the selection criteria used for admission to program (GPA, ACT, HESI A ${ }^{2}$ 
composite score) and on a critical thinking (CT) exam given at the beginning of the program.

Descriptive statistics for admission selection criteria and critical thinking scores by cohort are presented in Table 3. Results of the analysis of variance for each criterion are found in Table 4.

Table 3 Descriptive Statistics for Admission Criteria and Critical Thinking by Cohort

\begin{tabular}{lcccccccc}
\hline Cohort & \multicolumn{2}{c}{ GPA } & \multicolumn{2}{c}{ ACT } & \multicolumn{2}{c}{ A2 HESI } & \multicolumn{2}{c}{ CT } \\
\hline & Mean & SD & Mean & SD & Mean & SD & Mean & SD \\
\hline 1 & 3.26 & 0.44 & 22.48 & 2.17 & 75.43 & 5.13 & 836.97 & 44.76 \\
2 & 3.42 & 0.37 & 21.95 & 2.38 & 75.69 & 4.46 & 811.03 & 53.50 \\
3 & 3.27 & 0.45 & 22.20 & 2.62 & 78.81 & 5.31 & 784.67 & 43.62 \\
4 & 3.36 & 0.41 & 23.00 & 2.31 & 81.99 & 4.92 & 785.91 & 41.02 \\
5 & 3.22 & 0.49 & 22.23 & 2.18 & 81.30 & 4.49 & 767.73 & 56.90 \\
6 & 3.33 & 0.40 & 22.46 & 3.23 & 79.81 & 4.38 & 762.31 & 57.08 \\
\hline
\end{tabular}

Table 4 ANOVA Results for Admission Criteria and Critical Thinking by Cohort

\begin{tabular}{lrrrrr}
\hline & Sum of Squares & df & Mean Square & F & Sig. \\
\hline Adm. GPA & .835 & 5 & .167 & .921 & .469 \\
ACT & 17.779 & 5 & 3.556 & .565 & .727 \\
HESI A $^{2}$ & 1105.885 & 5 & 221.177 & 9.427 & .000 \\
Adm. CT & 118860.378 & 5 & 23772.076 & 9.749 & .000 \\
\hline
\end{tabular}


Differences in the means of the admission GPA ( 0.2 on a 4.0 scale) and ACT (1.05 points) for the six cohorts were non-significant. However, the difference in the mean scores of the HESI $A^{2}$ (6.56 points out of a possible 100) between the six cohorts was found to be significant at $\mathrm{F}(5,181)=9.427, p<.001$. In addition, differences in the mean score of the CT exam on admission to the program between the six cohorts were significant at $F(5,181)=9.749$, $p<.001$.

Polynomial contrasts were performed to determine the trend pattern of the HESI $\mathrm{A}^{2}$ and CT admission exam mean scores across the six cohorts. Significant polynomial contrasts identified of the HESI $\mathrm{A}^{2}$ mean scores were a positive linear trend at $\mathrm{F}(1,181)=32.278, p<$ .001 . In addition, the quadratic trend was significant at $\mathrm{F}(1,181)=6.390, p=.012$, as well as the cubic trend at $\mathrm{F}(1,181)=6.891, p=.009$. The only significant polynomial contrast identified of the CT admission exam mean scores was a negative linear trend at $\mathrm{F}(1,181)=$ 43.390, $p<.001$. Trend analysis of the HESI A2 mean scores and CT admission mean scores are depicted in Figure 2.

Figure 2 Trend Analysis of HESI $A^{2}$ and CT Admission Exam Means Across Cohorts

\section{Means Plots}
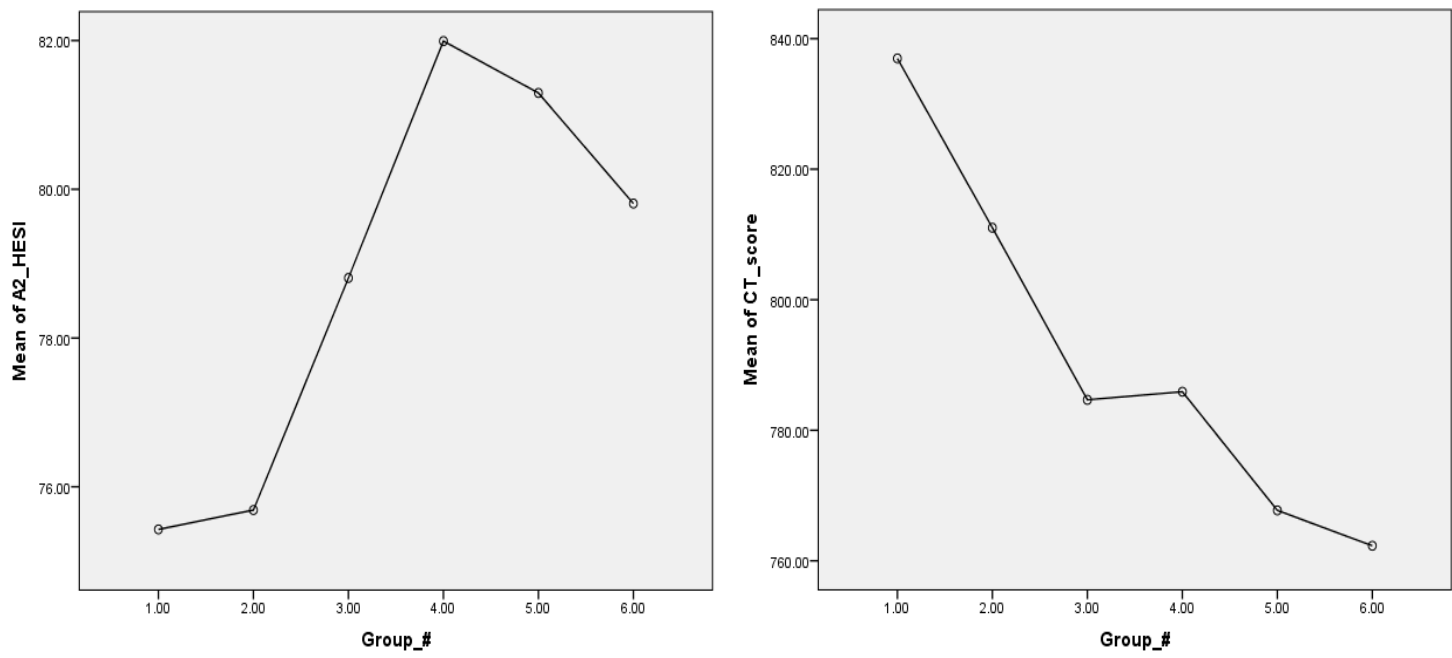
Pairwise comparisons of the HESI $\mathrm{A}^{2}$ exam mean scores and CT admission mean scores were done to describe the significant linear trend in more detail. Pairwise comparisons of the HESI A ${ }^{2}$ exam mean scores revealed that cohorts 1 and 2 had significantly lower HESI $\mathrm{A}^{2}$ mean scores than cohorts $3(p=.003, p=.004), 4(p<.001, p<.001), 5(p<.001, p<.001)$, and $6(p$ $=.001, p=.001)$. Additionally, the mean score of cohort 3 was significantly lower than the mean score of cohort $4(p=.012)$. This difference may have put these cohorts at a disadvantage when comparing the graduates ${ }^{\text {ee }}$ HESI $E^{2}$ mean scores on exit of the program. However, it was interesting to find that cohorts 1 and 2 had the highest mean scores on the admission CT exam of the six cohorts and the mean scores decreased with each graduating cohort. Cohort 1 , the control group with no exposure to HPS, had a significantly higher CT exam mean score on admission to program than cohorts $2(p=.028), 3(p<.001), 4(p<.001), 5(p<.001)$, and $6(p<.001)$. Furthermore, cohort 2 had a significantly higher CT admission exam score than cohorts 3 ( $p=$ $.016), 5(p=.001)$, and $6(p<.001)$. Cohort 6 , the group with the largest amount of exposure to HPS, had the lowest CT exam mean score on admission to program. These differences in the critical thinking abilities of the students upon admission to the program were accounted for by doing an analysis of covariance (ANCOVA) on the covariant of the CT exam score on admission to the program. Results of the pairwise comparisons between the six cohorts for the HESI $\mathrm{A}^{2}$ and admission CT exam mean scores are depicted in Table 5. 
Table 5 Pairwise Comparisons of HESI $A^{2}$ and CT Exam on Admission

\begin{tabular}{lcccccc}
\hline $\begin{array}{l}\text { Cohort } \\
\text { Contrasts }\end{array}$ & & \multicolumn{3}{c}{ HESI A2 } & & \multicolumn{3}{c}{ CT Admission Exam } \\
\hline & $\mathrm{T}$ & $\mathrm{Df}$ & Sig. (2-tailed) & $\mathrm{t}$ & $\mathrm{Df}$ & Sig. (2-tailed) \\
\hline 1v2 & -.227 & 181 & .821 & 2.221 & 181 & .028 \\
$1 \mathrm{v} 3$ & -3.045 & 181 & .003 & 4.622 & 181 & .000 \\
$1 \mathrm{v} 4$ & -4.925 & 181 & .000 & 3.757 & 181 & .000 \\
$1 \mathrm{v} 5$ & -4.402 & 181 & .000 & 5.095 & 181 & .000 \\
$1 \mathrm{v} 6$ & -3.449 & 181 & .001 & 5.766 & 181 & .000 \\
$2 \mathrm{v} 3$ & -2.945 & 181 & .004 & 2.440 & 181 & .016 \\
$2 \mathrm{v} 4$ & -4.882 & 181 & .000 & 1.908 & 181 & .058 \\
$2 \mathrm{v} 5$ & -4.342 & 181 & .000 & 3.288 & 181 & .001 \\
$2 \mathrm{v} 6$ & -3.360 & 181 & .001 & 3.897 & 181 & .000 \\
$3 \mathrm{v} 4$ & -2.528 & 181 & .012 & -.097 & 181 & .923 \\
$3 \mathrm{v} 5$ & -1.974 & 181 & .050 & 1.319 & 181 & .189 \\
$3 \mathrm{v} 6$ & -.838 & 181 & .403 & 1.838 & 181 & .068 \\
$4 \mathrm{v} 5$ & .478 & 181 & .633 & 1.221 & 181 & .224 \\
$4 \mathrm{v} 6$ & 1.557 & 181 & .121 & 1.650 & 181 & .101 \\
$5 \mathrm{v} 6$ & 1.060 & 181 & .291 & .379 & 181 & .705 \\
\hline
\end{tabular}

\section{Design}

The design for this study was an ex post facto design that used data over a span of six years from cohorts of students who have already graduated from an associate degree nursing program. This design was chosen to evaluate the impact of HPS when integrated across the curriculum of the nursing program of which I am the director. With the incorporation of HPS 
into the program of study, it was important to evaluate the impact of this teaching strategy on the student outcomes of critical thinking abilities and nursing knowledge.

Variables. The independent variable of the study was the cohorts that were exposed to variations in both the fidelity and the amount of exposure to HPS. Fidelity refers to the degree of realism the mannequin mimics and can be classified as low-fidelity (static and lacks the detail of realism), medium-fidelity (provides body sounds without corresponding movements), or highfidelity (mimics real physiology and patient-like functions). In addition, cohorts varied in the method of delivery and placement in the curriculum of HPS. Variations in the fidelity, the amount of exposure, method of delivery, and placement in the curriculum of HPS by cohort are depicted in Table 6.

Table 6 Variations in Fidelity, Exposure, Delivery, and Placement of HPS by Cohort

\begin{tabular}{llll}
\hline Cohort & Fidelity & Method of Delivery & $\begin{array}{c}\text { Semester Placement in } \\
\text { Curriculum }\end{array}$ \\
\hline 1 & Control group & No exposure to HPS & NA \\
2 & Medium-fidelity & $\begin{array}{l}\text { Two days of six hours each in } \\
\text { one week }\end{array}$ & $\begin{array}{l}\text { One week in the middle } \\
\text { of the fourth semester }\end{array}$ \\
4 & $\begin{array}{l}\text { Mixture medium- } \\
\text { and high-fidelity }\end{array}$ & $\begin{array}{l}1.5 \text { hours once a week for seven } \\
\text { or nine weeks }\end{array}$ & $\begin{array}{l}\text { Last seven or nine weeks } \\
\text { of the fourth semester }\end{array}$ \\
& High-fidelity & $\begin{array}{l}\text { Four sessions (one hour each) } \\
\text { Two hours every other week }\end{array}$ & $\begin{array}{l}\text { Second } \\
\text { Third and fourth }\end{array}$ \\
& High-fidelity & Two hours every three weeks & First \\
& & Two hours every other week & Second, third, fourth \\
6 & High-fidelity & Two hours every other week & All four semesters \\
\hline
\end{tabular}

Note. Simulation hours replaced traditional clinical hours. *Hours replaced campus lab hours. 
The dependent variables were nursing knowledge and critical thinking abilities. Critical thinking abilities and nursing knowledge, as measured by the Health Education Systems, Inc. (HESI) comprehensive exit exam $\left(\mathrm{E}^{2}\right)$, were compared for six cohorts of graduates exposed to differences in the fidelity and amount of HPS.

\section{Setting}

The setting for this study was a public community college situated on the outskirts of the third largest city in West Virginia and accredited to offer certificate and associate degree programs as well as a select few baccalaureate programs. The nursing program is a two year associate degree program which admits 40-48 students twice a year, in August and January. Upon completion of the program, students earn an associate of applied science degree in nursing and are eligible to sit for the NCLEX-RN for licensure as a registered nurse.

Program of study. The nursing program is designed to be completed in four semesters. Each semester spans 15 weeks of study plus a week of finals. The program of study is comprised of four nine-credit hour nursing courses, one taken each semester in the following sequence: Medical-Surgical Nursing I (NURS 111), Medical-Surgical Nursing II (NURS 112), Family Centered Care (NURS 213), and Medical-Surgical Nursing III (NURS 216). In addition to these four nursing courses, a one-hour physical assessment course (NURS 131) is included in the second semester; a two-hour perspectives of nursing (NURS 224) and a one-hour NCLEX review course (NURS 217) are taught in the fourth semester (see Appendix A for program of study).

The nine-credit nursing courses in the first two semesters of the program (NURS 111 and NURS 112) consist of three components: five hours of classroom theory, two hours of campus lab skills (one hour theory and demonstration and one hour scheduled practice), and nine hours 
of traditional clinical with patients per week. In the second year of the program, NURS 213 and NURS 216 encompass five hours of classroom theory and 12 hours of the traditional hospital based clinical per week. All psychomotor skills are taught in the first year of the program; no scheduled time in the campus lab is included in the courses of the second year of the program. NURS 131, 217, and 224 have no clinical component.

Simulation laboratory and simulators. All HPS experiences were conducted in the campus lab of the institution represented in the study. Prior to spring 2007, the campus lab involved two small adjoining rooms equipped with six Laerdal VitalSim mannequins and beds. The lab was used in NURS 111 and NURS 112 for the demonstration, practice, and evaluation of psychomotor skills. All skills were taught and the students evaluated by the full time faculty assigned to teach in these two courses. Although these mannequins are considered mediumfidelity simulators, they were initially not being used to the extent of their capabilities. Breath sounds were occasionally programmed for students to hear abnormal sounds; however, the mannequins were used primarily as static mannequins for skills practice and evaluation.

In 2007, a new campus lab was built that included six stations equipped with new beds, privacy curtains, headboards with suction and compressed air to simulate oxygen, sharps containers, supply carts, and a nurses ${ }^{e e}$ station. Additional equipment was bought that included pulse oximeters, dopplers, sequential compression devices (SCDs), bedside commodes, IV pumps, EKG machine, a defibrillator, and kangaroo pumps. One of the six stations in the corner of the room was sectioned off with glass walls to replicate a hospital room for a newly purchased Laerdal SimMan. A cardiac monitor was placed on a bedside stand beside the mannequin to view heart rate, blood pressure, and pulse oximetry. For recording of patient data throughout

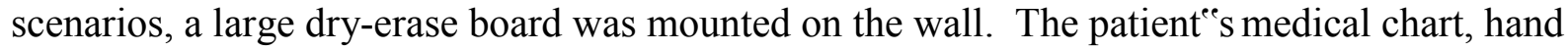


sanitizer to simulate hand washing, a clock to be set with the appropriate time, and resource reference books were available in the room. Video equipment was installed for recording the scenario. Recordings were available for use during the debriefing for students" review and critique. A table, used as a working station for the instructor and lab assistant, was set up outside the room with the laptop computer that controlled SimMan. An intercom system was installed between the glass room, the instructor workstation, and the student observation room to permit the instructor and students observing the simulation to hear the conversations of the students actively engaged in the HPS. The intercom was also used by the instructor to communicate information to students that could not be simulated, such as color and temperature of the skin. Additionally, telephones were installed between the simulated hospital room and the instructor"s workstation to allow for students to call physicians and other departments, such as the laboratory, pharmacy, operating room, or radiology departments, within a hospital setting.

With the support of administration, a METI iStan HPS was purchased summer 2008 and another glass enclosed room was added around one of the stations in the campus lab to simulate a hospital room for this mannequin. A third glass room was built for the purchase of the METI pediatric HPS. These rooms were equipped with the same features as the room with SimMan, complete with an intercom, telephones, video equipment, and faculty workstation.

\section{Instrumentation}

For the purpose of this study, the HESI $E^{2}$ exam was used as the tool to measure the dependent variables of nursing knowledge and critical thinking to answer the following research questions: 
1. How does nursing knowledge, as determined by the HESI $E^{2}$ score, of graduating associate degree nursing students trained with high-fidelity HPS compare to those trained with medium-fidelity HPS or those not exposed to HPS?

2. Is there a difference in knowledge, as determined by the $\mathrm{HESI} \mathrm{E}^{2}$ score, among the cohorts of graduating associate degree nursing students based on the number of hours traditional clinical experiences were substituted with HPS?

3. How do the critical thinking skills, as determined by the HESI E ${ }^{2} \mathrm{CT}$ score, of graduating associate degree nursing students trained with high-fidelity-HPS compare to those trained with medium-fidelity HPS or those not exposed to HPS?

4. Is there a difference in critical thinking skills, as determined by the HESI $\mathrm{E}^{2} \mathrm{CT}$ score, among the cohorts of graduating associate degree nursing students based on the number of hours traditional clinical experiences were substituted with HPS?

Description of the instrument. The HESI $E^{2}$, designed to measure the student"s nursing knowledge required for successful passage of the NCLEX-RN, was given to all nursing graduates towards the end of the final semester of the program. The HESI $\mathrm{E}^{2}$ is a highly reliable predictor of first time NCLEX-RN passage and the accuracy of the exam in predicting NCLEXRN success has been validated through eight validity studies (Young \& Langford, 2011). As noted by Young and Langford (2011), NCLEX-RN outcomes of over 43,000 students and 150 nursing programs across the United States have been reported in these studies with the predictive accuracy of the HESI $E^{2}$ ranging from $96.4 \%$ to $99.2 \%$ across all types of RN programs.

The HESI $E^{2}$ is a 160 -item comprehensive test, based on the NCLEX-RN test blueprint, designed to be given to students near the completion of the program of study (Morrison, Nibert, \& Flick, 2006). Of the 160 items, ten are pilot questions which are not scored. As described by 
HESI, scores of HESI exams can range from 0 to as high as 1,500 depending on the difficulty of the exam (Elsevier HESI Assessment, 2010). A score of 850 is considered an acceptable level of performance by Elsevier HESI. However, a score of 900 or above is the recommended score for predictability of success on NCLEX-RN. The overall student composite scores of the HESI E ${ }^{2}$ were used to measure the dependent variable of knowledge.

In addition to providing an overall composite score that assesses the nursing knowledge required for entry level practice, subject area scores are provided for over 50 content areas in the following categories: nursing process, client needs, specialty and sub-specialty areas, NLNAC accreditation categories, AACN curriculum categories; NLN educational competencies, quality and safety education (QSEN), and nursing concepts (Elsevier HESI Assessment, 2010). The test writing model, developed by Morrison, Nibert, and Flick (2006), is used with the development of each test item to evaluate critical thinking abilities within the discipline of nursing. All test items are written at the application and analysis level, requiring the student to think critically, use clinical judgment, and apply knowledge of course content to clinical practice situations. Critical thinking scores, included in the subject area of NLNAC Accreditation Categories and AACN Curriculum Categories, are provided that measure the student"s ability to use critical thinking skills necessary for clinical reasoning and judgment when selecting the correct answer. The nursing program represented in the study is accredited by the NLNAC; therefore, the critical thinking score reported in the NLNAC Accreditation Categories was used to measure the dependent variable of critical thinking.

Scoring. Students ${ }^{\text {ee }}$ overall scores and each subject area scores, used to determine students"e probability of passing the NCLEX-RN, are calculated by the application of the HESI Predictability Model (HPM) to the raw data (Morrison, Adamson, Nibert, \& Hsia, 2004). Based 
on the difficulty level, each test item is individually weighted by dividing the number of correct responses with the total number of item responses. A weighted percentage score, the conversion score, reflects the average weight of all the test items and items answered correctly. Reliability and item analysis calculations of HESI exams are based on the classical test theory: observed score equals true score plus error score. As noted by Morrison, Adamson, Nibert, and Hsia (2004), all test items are piloted prior to use. The parameters for a quality test item include a point biserial correlation co-efficient of 0.15 and above and no less than a $40 \%$ cumulative difficulty level.

Validity. Validity and reliability of HESI exams are determined on an ongoing basis. The degree of validity, as described in classical test theory, is determined through an assessment of construct, content, and criterion-related validity (Morrison et al., 2004). As discussed by Morrison et al. (2004), test items are evaluated for content validity by expert clinicians and nurse educators prior to the item being placed in the HESI test bank. The blueprint of each exit exam mimics the blueprint of the NCSBN and has been designed to test content relevant to entry-level nursing practice. Construct validity has been validated in studies comparing HESI exam scores with final course grades and cumulative grade point averages. The predictive accuracy of the HESI exams provides inferences about a student se $^{\text {s }}$ knowledge of nursing content and ability to succeed on the NLCEX-RN (Morrison et al., 2004).

Reliability. Reliability, measuring the consistency and accuracy of scores on the exam, is determined by conducting an item analysis of each exam given (Morrison et al., 2004). For every exam, a Kuder Richardson Formula 20 (KR-20) is calculated as a measure of reliability. The estimated reliability coefficients using KR-20 are determined every year for all four versions 
of the HESI Exit RN and ranged from 0.90 to 0.91 for 2009 and 0.90 to 0.92 for 2010 (Elsevier, Review \& Testing, 2010, 2011).

\section{Procedures}

The integration of simulation across the curriculum of our nursing program was not planned with the intent of a study in mind. One nursing faculty member, who demonstrated an interest in using HPS as a teaching strategy, was the underlying motivation for this curriculum change. Simulation with the use of medium-fidelity HPS was first introduced into the program fall 2006. By fall 2008, high-fidelity HPS was integrated across the curriculum. The purpose of this study was to examine data from the last six years to determine the impact of this curriculum change on students ${ }^{e e}$ critical thinking skills and knowledge gain.

Introduction of medium-fidelity HPS. The lead faculty member of NURS 216, the advanced medical-surgical course taken the last semester of the program, was the first faculty member to take an interest in using the Laerdal VitalSim mannequins for a scenario-based HPS. During the 2006/2007 academic year, this faculty member incorporated a combined skills/simulation campus lab experience into NURS 216. In lieu of one week of clinical (two six-hour days), students enrolled in the course attended the campus lab for a combined skills/simulation experience. Each student participated in two medium-fidelity human patient simulations each day for a total of four simulations The four scenarios were self-created by the faculty member and were based on common situations the students are required to handle in the clinical setting (see Appendix B for scenarios). This cohort of students, May 2007 graduates, was the first to participate in medium-fidelity HPS substituted for 12 hours of traditional clinical time. 
Introduction of high-fidelity HPS. The following year, with the renovation of the new campus lab and the purchase of Laerdale's SimMan, experiences with HPS were expanded in NURS 216 to include a mixture of both medium- and high-fidelity HPS. As opposed to having one week of clinical substituted with HPS in the campus lab, traditional clinical hours were shortened by one and a half hours per week for the last seven to nine weeks of the semester. During those weeks, students traveled back to campus for participation in a one and a half hour skills/simulation experience in the campus lab. Each week, students rotated between four skill stations and participated in a medium-fidelity HPS with a Laerdal VitalSim or a high-fidelity HPS with SimMan, alternating each week. The four scenarios used the previous year were used in addition to others that were purchased with SimMan (see Appendix C for scenarios). A different scenario was presented each week and was used with both the medium- and highfidelity mannequins. The lead faculty member, who initiated the simulation experiences, conducted all the high-fidelity HPS scenarios. A full time faculty member, who was team teaching in the course, conducted the medium-fidelity HPS scenarios. Graduates of this cohort, May 2008, participated in four or five medium-fidelity simulations and three or four high-fidelity HPSs.

The program was fortunate in that the faculty member who first incorporated simulation into her course, NURS 216, became our champion faculty member promoting the integration of HPS across the curriculum. In addition to adding the skills/simulation campus lab in lieu of clinical time, she worked with the lead faculty of NURS 111 and NURS 112 to incorporate simulation into their courses. Spring 2008, four campus lab one-hour practice sessions in NURS 112, spaced throughout the semester, were devoted to HPS. During these labs, each student participated in a HPS with SimMan. That same semester, faculty of NURS 111 decided to pilot 
substituting clinical time with HPS. The last eight weeks of clinical were reduced by one hour per week and substituted with HPS. Starting the fourth week of the semester, students attended a two-hour simulation lab every three weeks using SimMan for a total of four HPS experiences during the semester (see Appendix $\mathrm{C}$ for scenarios).

Integration of HPS across the curriculum. The faculty unanimously voted to incorporate HPS across the curriculum substituting a small percentage of traditional clinical hours for simulation. A proposal was sent to the West Virginia Board of Examiners for Registered Professional Nurses (WVBRN) in 2008 and approval was granted to substitute no more than $20 \%$ of clinical time with HPS. Effective fall 2008, our champion faculty ${ }^{\text {ee }}$ workload was devoted $100 \%$ to simulation. Traditional clinical hours were reduced by one hour per week for the last twelve weeks of the first semester course (total of 12 hours) and beginning week four, students attended a two-hour simulation lab every other week for a total of six HPS experiences. In the remaining three semesters, traditional clinical hours were reduced by one hour per week for all 15 weeks (total of 15 hours). As a replacement for these hours, students were assigned to a two-hour simulation lab that met every other week for a total of seven simulation labs. In addition, students were required to complete a one-hour self-study review of the orientation materials each semester. Table 7 describes the fidelity of HPS, the amount of exposure to HPS, and the number of hours HPS was substituted for traditional clinical experiences for each cohort.

Scheduling. The simulation instructor developed the simulation schedule and identified lab times for each course that would fit around the classroom and clinical schedule. Students within each course were assigned their lab time by the simulation instructor. The groups stayed together for an entire semester; however, groups were mixed in subsequent semesters. Students were randomly assigned to groups of six for the first semester course, NURS 111. For the 
Table 7 Fidelity and number of HPS and Hours Replaced for Clinical per Cohort

\begin{tabular}{|c|c|c|c|c|}
\hline $\begin{array}{c}\text { Graduate } \\
\text { Cohort }\end{array}$ & $\begin{array}{l}\text { NURS } \\
\text { Course }\end{array}$ & $\begin{array}{c}\text { Number and } \\
\text { fidelity of HPS }\end{array}$ & Frequency of Delivery & $\begin{array}{l}\text { Hours HPS substituted } \\
\text { for traditional clinical }\end{array}$ \\
\hline $\begin{array}{l}\text { Cohort } 1 \\
5 / 2006\end{array}$ & $\begin{array}{l}111,112 \\
213,216\end{array}$ & 0 & & 0 \\
\hline \multirow[t]{2}{*}{$\begin{array}{l}\text { Cohort } 2 \\
5 / 2007\end{array}$} & $\begin{array}{c}111,112 \\
213\end{array}$ & 0 & & 0 \\
\hline & 216 & 4 MFHPS & 1 week of two $(6 \mathrm{hr})$ days & 12 \\
\hline \multirow[t]{2}{*}{$\begin{array}{l}\text { Cohort } 3 \\
5 / 2008\end{array}$} & $\begin{array}{c}111,112 \\
213\end{array}$ & 0 & & 0 \\
\hline & 216 & $\begin{array}{l}3 \text { or } 4 \text { MFHPS } \\
4 \text { or } 5 \text { HFHPS }\end{array}$ & $\begin{array}{c}11 / 2 \text { hours per week for } \\
7 \text { or } 9 \text { weeks }\end{array}$ & $10.5-13.5$ \\
\hline \multirow{3}{*}{$\begin{array}{l}\text { Cohort } 4 \\
5 / 2009\end{array}$} & 111 & 0 & & 0 \\
\hline & 112 & 4 HFHPS & 1 hour $\mathrm{x} 4$ in campus lab & 0 \\
\hline & 213,216 & 7 HFHPS & 2 hours every other week* & 30 \\
\hline \multirow{2}{*}{$\begin{array}{l}\text { Cohort } 5 \\
12 / 2009\end{array}$} & 111 & 4 HFHPS & 2 hours every third week ${ }^{* *}$ & 8 \\
\hline & $\begin{array}{c}112,213 \\
216\end{array}$ & 7 HFHPS & 2 hours every other week* & 45 \\
\hline \multirow{2}{*}{$\begin{array}{l}\text { Cohort } 6 \\
5 / 2010\end{array}$} & 111 & 6 HFHPS & 2 hours every other week** & 12 \\
\hline & $\begin{array}{c}112,213, \\
216\end{array}$ & 7 HFHPS & 2 hours every other week* & 45 \\
\hline
\end{tabular}

Note. MFHPS = medium-fidelity human patient simulation; HFHPS = high-fidelity human patient simulation. *Students completed orientation materials on-line for one hour; ${ }^{*}$ HPS started week 4 of the semester. 
remaining courses, the simulation instructor ${ }^{\text {ee }}$ goal was to group the students in groups of eight. This was not always possible and depending on course enrollment groups varied from seven to ten students per group. As the semester progressed, group sizes frequently decreased as students withdrew from courses. After the first semester course, students were grouped according to ability. Stronger students were assigned together, as well as weaker students. The rationale for grouping by ability was to ensure that those students who did not always excel in the classroom and/or clinical setting had the opportunity to problem solve and make decisions without stronger students taking over.

Policies. Simulation lab hours were considered clinical hours and attendance was mandatory for all students. To assist the student in assuming the professional role of the nurse (Childs \& Sepples, 2006) and add to the reality of the experience (Bambini et al., 2009; Limoges, 2010), the nursing uniform was required to be worn during simulation. Procedures in place for traditional clinical hours were also enforced with the simulation hours. Students were expected to arrive on time, in full uniform, with their prep work completed. Failure to do so resulted in an unsatisfactory simulation experience and students were sent home. One missed simulation experience each semester could be made up; a second missed simulation was considered excessive and the student was required to withdraw from the course.

NLN's nursing education simulation framework. The National League for Nursing ${ }^{\text {ee }} \mathrm{s}$ (NLN) Nursing Education Simulation Framework was used as the organizing framework to guide the design and implementation of HPS into the curriculum of the nursing program (Jeffries, 2007). As described in chapter one, the model depicts five conceptual components, each with specific variables that are associated with and influence the outcomes of a simulation. 
These five components include: teacher, student, educational practices, simulation design characteristics, and outcomes.

Teacher. The simulation instructor assumed the role of facilitator and evaluator throughout the simulation experiences. The instructor was situated outside of the room and played several roles: the voice of the patient, the physician, or other interdisciplinary team members that the students called. Cues, such as patient statements and physician comments or questions, were used if students were missing pertinent data or to redirect students to keep the scenario on track for the accomplishment of the objectives (Garrett, MacPhee, \& Jackson, 2010). The lab manager was responsible for setting up the mannequin prior to the simulations, running the computer that operates the mannequin, and operating the audio-visual equipment for videotaping throughout the simulation. Occasionally, the lab manager provided patient data through the intercom, answered the telephone to give lab or radiology reports, or played the role of a family member. However, the simulation instructor always assumed the role of the physician and the voice of the patient.

In addition to many hours of professional self-development, the simulation instructor attended several training sessions and workshops that provided her with the knowledge and skills to be comfortable with the use of simulation as a teaching strategy and the technology involved. She attended training sessions offered by the manufacturers for SimMan and iStan. In addition, the simulation instructor has attended two of the national and one of the regional Human Patient Simulation Network (HPSN) conventions offered by METI and one of the annual Peter M. Winter Institute for Simulation Education and Research (WISER) Nursing Symposiums.

Student. The HPS experiences were student-centered and required students to take responsibility for their own learning. In addition to the hands-on orientation to the mannequin 
during the first half-hour of the initial simulation, each student was required to complete an online self study that included the ground rules for simulation, instructions on how to use the mannequins, descriptions and responsibilities of each student role, and student expectations (see Appendix D for orientation materials). Students were expected to review these at the beginning of each semester. Orientation to the mannequin and expectations and ground rules of the simulation facilitates student buy-in, fosters realism, and promotes student investment in the learning experience (Hawkins, Todd, \& Manz, 2008). Additionally, a thorough orientation eliminates distractions and permits the student to focus on the objectives of the simulation (Jeffries, 2005). A consent form for videotaping and a confidentiality agreement was signed by each student prior to the first simulation lab.

Students were expected to come prepared to the simulation laboratory with a prep worksheet completed (see Appendix E for sample prep sheets). The prep sheets, developed by the simulation instructor, were designed to prepare the student with general knowledge about the patient"s condition and skills in the scenario to enable the student to work through the scenario. Patient specific data were not included in the prep sheets. The simulation instructor wanted to keep some element of surprise in the simulation to prepare the student for actual practice. Students were permitted to use their prep sheet as a resource during the scenario (Waxman, 2010).

Upon arrival to the simulation lab, the group of six to ten students assigned to that lab was divided into two subgroups by the simulation instructor. Group one was assigned to the process-based role and completed the first scenario, while group two was assigned to the response-based role and observed the scenario from outside the room. By assigning only six students to each simulation lab for the first semester course and eight students for the remaining 
semesters, these two groups were kept to three or four students participating in a scenario. On the occasions when nine or ten students had to be assigned in the second year courses, up to five students participated in a scenario. Although the literature supports that up to four students is the ideal number for group simulation (Childs \& Sepples, 2006), simulations can involve up to six students (Jeffries, 2008). The scenarios in the second year of the program were complex enough to involve all five students. Furthermore, with grouping students by ability, weaker students were not able to step back and let the stronger students take over. Each week students were mixed when assigning the two subgroups to ensure that each student had equal time being in the first simulation group and opportunity to work in a team with different students. This was important to provide each student with opportunities to share information, delegate, develop communication skills, and promote collaborative learning and team work (Brewer, 2011; Jeffries, 2005).

Students were assigned specific roles for the simulation by the simulation instructor. Roles were switched every lab to ensure that each student had opportunity to participate in all roles over the course of the semester. The roles for the first semester course included: primary nurse, treatment/medication nurse, and communicator/recorder. Roles for the other three courses included: primary nurse, medication nurse, treatment nurse, and communicator/recorder. For the simulation groups of nine or ten that required five students to participate in the scenario, the role of secondary nurse was added. A card with the identified role was worn around the studentes neck throughout the simulation (See Appendix D for orientation materials with a description of roles).

After roles were assigned, group one was given a patient report typed on a card. Typically the student assigned to the primary nurse role read the card to the rest of the group. 
The group was given five minutes to discuss the patient and develop a plan prior to moving into the simulation. Allowing time for students to plan and prepare is important for the success of the simulation experience (Garrett et al., 2010; Jeffries, 2005). One area of focus during the debriefing was a discussion about how the group utilized this planning time. Many groups were task oriented and used planning time to focus on identifying what tasks needed to be completed and who would do them. The instructor used time during debriefing to assist students in learning how to think and use the nursing process to create a mental concept map of patient needs, actual and potential problems, pertinent assessments that are essential, and interventions. The nursing process and concept mapping are introduced early in the first course of the semester. For simulation to be effective in teaching thinking skills, not only must skills be taught and modeled by the instructor, but students must also be given the opportunity to practice skills with feedback (Su \& Juestel, 2010).

Throughout the simulation, students were expected to perform assessments and interventions following the principles of safety: hand washing, patient identifiers, medical asepsis, six rights of medication administration, reading back verbal orders, etc. The use of Situation, Background, Assessment, and Recommendations (SBAR) communication was required as the method of communication with members of the interdisciplinary team. Opportunity to call a physician was incorporated into every simulation. On the occasions when scenarios were moved ahead in time for the second group, the first group was required to give an oral report on the patient's condition.

Differences of opinion exist in the literature regarding the length of time that should be allotted to a scenario with HPS (Garrett et al., 2010) and lengths varied from 15 minutes to one hour. The simulation instructor believed it was important that students be given adequate time to 
accomplish the objectives of the simulation (2010; Childs \& Sepples, 2006). Therefore, more latitude was typically given to group one to complete the scenario. In general, the second group was able to move through the scenario a little quicker, requiring less time. When group one either met the objectives of the simulation or a maximum of 50 minutes had passed, the two groups switched roles. Group one became the observers while group two repeated the scenario. Fifty minutes was chosen as the maximum length of time for group one to complete the scenario to allow for at least 15-20 minutes for group two to complete the scenario and 30 minutes for debriefing.

Occasionally, a scenario was moved forward to a different point in time or changed slightly from the original scenario for group two. For example, the scenario with diabetic ketoacidosis was modified for the second group. Although it was the same patient with the same history and background, the patient experienced hypoglycemia versus hyperglycermia.

At the completion of the second scenario or when time expired, all students in groups one and two moved to the debriefing room located down the hall from the simulation lab. A minimum of the last 30 minutes of the two-hour simulation lab was dedicated to debriefing. All students were expected to participate in a self-assessment of their performance and ability to meet the learning outcomes of HPS.

Educational practices. Students assumed an active role and participated in HPS scenarios during each simulation lab. While assuming the passive role of the observer, students were provided with a worksheet and were expected to take notes on the performance of the group of students doing the simulation. The simulation created a safe environment for the students to make mistakes; the students were expected to make decisions and take action, even the wrong action, without the interference of the simulation instructor. Occasionally, the simulated patient 
died as a result of the decisions made by the students. Other than cues that were provided to keep students on track and the scenario moving forward, the instructor did not interrupt the scenario to offer assistance or provide feedback to students. Interrupting the scenario or assisting students in the decision-making process takes away from problem solving and decision-making attempts by the students (Jeffries, 2008) and can impede student learning (Hawkins, et al., 2008). Feedback was given in the debriefing room immediately after the two groups completed the scenarios.

Activities were incorporated into each scenario to meet the needs of visual, auditory, tactile, and kinesthetic learners. For the visual learner, the simulation room closely resembled a hospital patient room complete with suctioning, compressed air for oxygen, cardiac monitoring, telephone, and appropriate equipment for the scenario. The patient ${ }^{\mathrm{ee}} \mathrm{s}$ chart, complete with physician orders, laboratory and radiology reports, flow sheets, nursing notes, and other documents pertinent to the scenario; was available in the simulation room. The clock in the room was set to the time of the simulation and the students were in complete uniform. A large cart with supplies simulating a hospital supply room and a medication cart were outside the patiente's room. Having the ability to auscultate breath sounds, bowel sounds, and blood pressures; use a telephone to simulate telephone calls to the physician or other departments; and use the microphone that transmitted the voice of the mannequin accommodated the auditory learner. Tactile and kinesthetic learners had opportunities to touch the mannequin to obtain blood pressure and palpate pulses; auscultate heart, lung, and bowel sounds; handle equipment such as IV pumps, suction and oxygen controls, doppler, and pulse oximeter; and perform psychomotor skills such as medication administration, starting an IV, changing a dressing, inserting a nasogastric tube, or inserting a urinary catheter. 
Although the simulation instructor attempted to create a supportive, collaborative, and positive environment within the simulation laboratory experiences, students initially reported high anxiety with the experience. They often felt intimidated by the simulation instructor and the wealth of her knowledge. However, it has been noted by the simulation instructor that as students are exposed to simulation over the course of the program, they become less anxious and more appreciative of the value of simulation.

Students were expected to do their best in the simulation learning experiences. The simulation instructor provided support and guidance during the debriefing sessions to facilitate students ${ }^{\text {ee }}$ learning. To decrease the anxiety associated with being evaluated and to provide an environment where students felt safe to act upon their decisions, the simulation laboratory was not graded. However, the simulation instructor did identify students who were weak in certain areas and these weak areas were discussed with the student, the course instructor, and the clinical instructor. Areas of weakness within the curriculum have also been identified and were communicated to the faculty of the appropriate course. This information has assisted faculty as they evaluated and made changes to their courses.

Simulation design characteristics. A properly designed scenario is essential for achieving the outcomes of student satisfaction, self-confidence, critical thinking, knowledge, and performance (Waxman, 2010). Variables that need to be considered when designing a scenario include: objectives, fidelity, problem solving, student support, and reflective thinking/debriefing (Jeffries, 2007). To ensure that each scenario was well designed, validated, and evidence-based, the nursing program bought the Laerdal (2007) NLN Simulation in Nursing Education with the purchase of SimMan and METI ${ }^{\text {ee }}$ Program for Nursing Curriculum Integration (PNCI) with the purchase of iStan. The NLN Simulation in Nursing Education contained surgical and medical 
scenarios, ranging in complexity, which could be used with SimMan. These scenarios came with patient kardex, physician orders, and data collection forms (Laerdal, 2012b). The PNCI included 100 simulated clinical experiences (SCE), more commonly referred to as scenarios, which focused on nursing educational concepts and competencies (Nehring \& Lashley, 2010). Each SCE was presented in a standard format, was available in an electronic document for customization by faculty, and included: an overview and synopsis of the SCE; learning objectives with the cognitive taxonomy and NCLEX-RN test plan categories; scenario with patient events, pertinent data, cues and questions, and behaviors minimally expected of the student; history and background information of the patient; physician "s orders; questions for students to prepare for the SCE; and the equipment, supplies, and setup needed with notes to assist faculty. In addition to $100 \mathrm{SCE}^{e e}$, the PNCI included a debriefing guide, SBAR communication guide, and evaluation forms to assist faculty (2010).

The simulation instructor met with a group of faculty, representing all courses, to select the types of diagnoses, patient conditions, and skills that would be covered with scenarios. Scenarios were chosen from both the NLN Simulation in Nursing Education and the PNCI based on the objectives of the scenario that would best meet the intended desired outcomes of the experience and course content. Scenarios were modified by the simulation instructor to match the complexity of the scenario with the level and abilities of the student (Rauen, 2001). When planning the schedule for simulation, the simulation instructor worked closely with the lead course instructors so that scenarios would be closely aligned with the presentation of the content in the classroom theory portion of the course. This ensured that students had been presented with the knowledge and skills necessary for effective problem solving and decision-making prior to the SCE. Scheduling SCEs before the content and key concepts are presented in class can 
lead to unsuccessful experiences resulting in negative feelings and decreased self-confidence of students (Prion, 2008).

The objectives were clearly identified for each SCE and utilized to guide the debriefing. However, in contrast to Jeffries ${ }^{\text {ee }}(2008)$ suggestion, objectives were not shared with the student prior to the simulation lab. Differences of opinion exist among nurse educators regarding the value of communicating the learning objectives to students prior to the simulation experience (Prion, 2008). The objectives for our simulations were very specific and it was determined that communicating these to students would provide too much information about the scenario. Although it was important that students were adequately prepared with the general knowledge necessary for the successful completion of the simulation, the instructor did not want to "give away" the situation. Therefore, prep sheets were carefully designed to include general information needed without giving away key events of the scenario (see Appendix E for sample prep worksheets).

Once simulation was fully integrated across the curriculum, each student participated in 27 scenarios throughout the two years of the program (see Appendix F for scenarios and objectives). Since the time frame of this study ended, the total number of scenarios has been reduced to 26. It was determined that attempting to orient students to the mannequin during the first simulation was not effective in preparing students for HPS. Therefore, the first two-hour simulation lab of the first semester course is now devoted entirely to orientation, with opportunities for the student to practice with the mannequins.

HPS was integrated into our curriculum for the purpose of standardizing experiences for all students and providing a safe environment for students to participate in critical events they may not be exposed to in the traditional clinical setting. Although the literature suggests that 
low-fidelity mannequins are more appropriate for the beginning student while more advanced students benefit from high-fidelity HPS (Jeffries, 2008), high-fidelity mannequins (Laerdale"s SimMan and METI"s iStan) were used for all simulation labs since fall 2008. Psychomotor skills were included with the majority of the scenarios; however, the primary goal of implementing HPS was to develop communication skills, critical thinking, decision-making, and problemsolving abilities of our students. Students in the first year of the program (NURS 111 and NURS 112) continued to be scheduled one hour per week in the skills lab for practice of skills on the VitalSim mannequins, in addition to the simulation lab with HPS in lieu of clinical time. When planning the scenarios to be used with beginning students, the level of difficulty of the HPS was adjusted to their level. However, the faculty made sure each scenario required appropriate assessments and some level of problem-solving to work through a particular situation. HPS in the second, third, and fourth semesters progressively became more complex, involving patients with an increasing number of problems requiring decision-making, clinical judgments, anticipation of patient needs, and prioritization of care.

Cues were provided throughout the scenario as identified in the NLN Simulation in Nursing Education and PNCI. Opportunities for students to self-reflect were provided in the debriefing session. Debriefing occurred immediately after the simulation laboratory so information and student feelings that were experienced during the simulation would not be lost (Johnson-Russell \& Bailey, 2010; Waxman, 2010). Guidelines offered by Decker (2007a) served as a resource for conducting the debriefing session. It was emphasized to students that SCEs and the debriefing that followed were a learning experience and would not be graded. A debriefing room, away from the simulation laboratory, was constructed and offered a private, quiet, comfortable environment for the debriefing (Decker, 2007a; Johnson-Russell \& Bailey, 
2010). Table and chairs in the room permitted students and the instructor to sit around the table as peers. All students assigned to the simulation laboratory participated in the debriefing.

The simulation instructor led the debriefing and served as facilitator by asking questions to stimulate thinking and encourage student reflection on their performance versus lecturing to the group and providing all the information (Johnson-Russell \& Bailey, 2010). The discussion was started with the instructor asking an open-ended question such as "How do you feel things went?" (Brewer, 2011; Johnson-Russell \& Bailey, 2010; Waxman, 2010). The objectives of the scenario were used to guide the discussion and students were asked to reflect on how well they perceived they were prepared for accomplishing the objectives and if the objectives were met. Students need guidance and role modeling by the faculty to develop the critical thinking processes needed for clinical reasoning and problem solving (Facione \& Facione, 2008). Students were asked during debriefing to provide rationales for their decisions, consider multiple possibilities, explore alternatives, and evaluate the consequences of alternative actions (Medley $\&$ Horne, 2005). Instructor guidelines for the debriefing session and suggested questions for students were included in the faculty worksheet for each SCE (Garrett et al., 2010).

An environment perceived to be safe to discuss feelings without being ridiculed or criticized by faculty or peers is essential (Johnson-Russell \& Bailey, 2010). To promote trust within the group, students signed a confidentiality statement ensuring that comments and events surrounding the simulation experience would not be shared outside the group (Decker, 2007a; Johnson-Russell \& Bailey, 2010; Waxman, 2010). All group members were encouraged to selfreflect, engage in the discussion, and share their feelings. Each scenario was videotaped and transmitted to a monitor in the debriefing room for viewing only by those students assigned to that simulation lab. Portions of the video were viewed when the instructor wanted to emphasize 
a key point. As students self-reflected on their performance, opportunity to review parts of the video helped students to remember what actually happened, as opposed to what they perceived had happened.

\section{Data Collection}

Data for this study were data normally collected by the nursing program and saved in the Health Sciences official student data base for educational purposes and program assessment needs required for national accreditation. For the protection of the rights of human subjects, the study was submitted to West Virginia Universityes Institutional Review Board and the research study was granted an exemption in accordance with "research on normal educational practices" (Appendix G). Prior to data collection, approval to use the data for research purposes was obtained from the Vice President of Academic Affairs of the community college offering the nursing program (Appendix H). In addition, the Nurse Researcher Agreement to Participate in Elsevier/HESI Educational Research Projects form was signed and submitted to Elsevier/HESI (Appendix I) prior to the collection of student scores of the HESI A ${ }^{2}$, Critical Thinking exam, and HESI $E^{2}$ for research purposes.

After appropriate approvals were granted, data for the six cohorts of students, representing the sample for the study, were obtained from the Health Sciences official data base and Elsevier"s Director of Research. An excel spreadsheet was created to record the data of the six cohorts. Data collected were demographics, admission data, and HESI $\mathrm{E}^{2}$ overall scores and HESI $E^{2}$ CT scores. To maintain confidentiality, no student identifiers were included in the spreadsheet that was used for this study.

Description of data. In addition to demographic data (age, gender, and ethnicity), date of admission (identified by cohort number) and data required for admission to program (ACT, 
cumulative grade point average [GPA], and HESI Admissions Assessment $\left[\mathrm{A}^{2}\right]$ composite score) were collected for the purpose of comparing homogeneity among the cohorts at the beginning of the program. The HESI $\mathrm{A}^{2}$ exam is designed to be used as an assessment of a student"s academic and personal readiness for nursing. Academic areas of grammar, vocabulary, reading comprehension, math, chemistry, biology, and anatomy and physiology, in addition to the Learning Styles Inventory and Personality Profile, make up the $\mathrm{A}^{2}$ exam (Siefert, Hinds, Yoho, Nibert, \& Morrison, $n . d$.). The composite (average) score of the seven academic exams was used for this study.

The HESI CT exam, required to be taken by all students within the first three weeks of the program, is given to assess the critical thinking abilities of the students upon admission to the program. The 25-item multiple-choice exam, designed to reflect a student's critical thinking ability in situations commonly found in health care, provides a total score for the exam (Siefert et al., n.d.). As described by Siefert, Hinds, Yoho, Nibert, and Morrison (n.d.), a Likert-type scale is used for scoring. All answers are correct, but the answer requiring the highest level of critical thinking is given the maximum weight. Scores can range from 0-1000 and the higher the score, the better the student"s ability to think critically within the discipline of nursing. The estimated reliability coefficients, calculated by KR-20, ranged from $0.87-0.99$. Students" total scores on the exam were collected and used to identify and compare the critical thinking abilities of the participants in each cohort upon admission to the program. An analysis of covariance was done

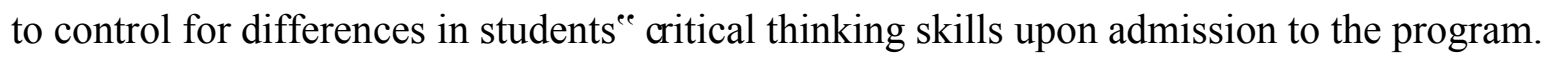

The HESI $E^{2}$ scores of individual students, used to measure the dependent variable of knowledge, were collected from the Health Sciences database. Individual students ${ }^{\text {ce }} \mathrm{HESI} \mathrm{E}^{2} \mathrm{CT}$ scores, used to measure the dependent variable of critical thinking, were not included in the 
Health Sciences database, but were accessible to the program through Elsevier"s HESI testing website. The HESI exam scores prior to 2009 had been archived by Elsevier and were inaccessible through their website. Therefore, individual students" $\mathrm{HESI} \mathrm{E}^{2} \mathrm{CT}$ scores, prior to 2009, were obtained from the director of research for Elsevier"s Review and Testing/HESI.

\section{Statistical Analysis}

Data were analyzed using the Statistical Package for the Social Sciences (SPSS) Version 20. Simple descriptive statistics were used to determine the frequency and percentage of gender and ethnicity, as well as mean and standard deviation for age. To measure for homogeneity of cohorts, admission criteria (ACT, GPA, and HESI $\mathrm{A}^{2}$ scores) and HESI CT exam scores on admission were analyzed using descriptive statistics: mean, standard deviation. To determine if differences between the six cohorts were significant, an ANOVA was performed on each of the admission criteria variables to compare the means of the six cohorts. When significant statistical differences in the mean scores were found by the ANOVA, polynomial contrasts were utilized to describe the pattern of those differences across cohorts and pairwise comparisons of the mean scores between each cohort were performed to further describe significant statistical differences.

For this study, cohort which was defined by the type of fidelity and amount of exposure to HPS was the independent variable and knowledge and critical thinking were the dependent variables. To address each research question and determine the effects of the fidelity and amount of student exposure to HPS on critical thinking and knowledge, analyses compared the mean scores of the HESI $E^{2}$ and HESI $E^{2} \mathrm{CT}$ of the six cohorts of graduates. Statistical significance for the study was set at the $p<.05$.

Violations of assumptions. As a retrospective study, group sizes were determined by the number of students within each cohort graduating within the prescribed two years. With the 
unequal group sizes that ranged from 22 to 45, testing for violations of the assumptions of normality and homogeneity of variance was completed. Assessing if HESI $\mathrm{E}^{2}$ and $\mathrm{HESI} \mathrm{E}^{2} \mathrm{CT}$ exam scores were normally distributed within each of the six cohorts to examine for the assumption of normality was conducted with the Shapiro-Wilk test of normality. Homogeneity of variance across all six cohorts was tested with the Levene $e^{e e}$ test. The observed power of the sample sizes were examined during the analysis.

Research questions 1 and 2. Single-factor, independent-measures, one-way analysis of variance (ANOVA) was performed to test for significant statistical mean differences of HESI $E^{2}$ scores among the six cohorts of graduates to answer the following research questions:

1. How does nursing knowledge, as determined by the HESI $E^{2}$ score, of graduating associate degree nursing students trained with high-fidelity HPS compare to those trained with medium-fidelity HPS or those not exposed to HPS?

2. Is there a difference in knowledge, as determined by the HESI $E^{2}$ score, among the cohorts of graduating associate degree nursing students based on the number of hours traditional clinical experiences were substituted with HPS?

Research questions 3 and 4. Single-factor, independent-measures, one-way analysis of variance (ANOVA) was performed to test for significant statistical mean differences of HESI $\mathrm{E}^{2}$ CT scores among the six cohorts of graduates to answer the following research questions:

3. How do the critical thinking skills, as determined by the HESI $\mathrm{E}^{2} \mathrm{CT}$ score, of graduating associate degree nursing students trained with high-fidelity HPS compare to those trained with medium-fidelity HPS or those not exposed to HPS? 
4. Is there a difference in critical thinking skills, as determined by the HESI $E^{2} \mathrm{CT}$ score, among the cohorts of graduating associate degree nursing students based on the number of hours traditional clinical experiences were substituted with HPS?

Polynomial contrasts and pairwise comparisons. When significant statistical differences in the mean scores were found by the ANOVA, polynomial contrasts were utilized to describe the pattern of those differences across cohorts. Patterns identified with this trend analysis determined whether the trend across cohorts appeared to be linear or curvilinear. Post hoc pairwise comparisons of the mean scores were performed to describe significant trends in more detail with no attempt to control overall Type 1 error rate.

Research question 1 and 2. Pairwise comparisons of the HESI $\mathrm{E}^{2}$ mean scores between each cohort were performed to further describe which pairs of cohorts had significant statistical differences in mean scores of knowledge. Results identified by these pairwise comparisons were used to evaluate the relationship between the fidelity of and the number of hours of simulation that was substituted for traditional clinical experiences (independent variable) and knowledge (dependent variable) to test for the following hypotheses:

- A significant increase will be seen with knowledge gain, measured by the HESI $\mathrm{E}^{2}$ score, as cohorts are exposed to an increased fidelity of HPS.

- A significant, positive linear trend will be seen with knowledge gain, measured by the HESI $E^{2}$ score, as cohorts are exposed to an increased number of hours that HPS is substituted for traditional clinical experiences.

Research question 3 and 4. Pairwise comparisons of the HESI E ${ }^{2}$ CT mean scores between each cohort were performed to further describe which pairs of cohorts had significant statistical differences in mean scores of critical thinking. Results of the pairwise comparisons 
were used to evaluate the relationship between the fidelity of and the number of hours of simulation that was substituted for traditional clinical experiences (independent variable) and critical thinking (dependent variable) to test for the following hypotheses:

- A significant increase will be seen with critical thinking, measured by the HESI E ${ }^{2}$ CT score, as cohorts are exposed to an increased fidelity of HPS.

- A significant, positive linear trend will be seen with critical thinking skills, measured by the HESI $\mathrm{E}^{2} \mathrm{CT}$ score, as cohorts are exposed to an increased number of hours that HPS is substituted for traditional clinical experiences.

Analysis of covariance. Analysis of covariance (ANCOVA) was done on the covariant of critical thinking abilities on admission to program. Critical thinking skills of students on admission were measured by the score on a critical thinking exam given at the beginning of the program. This analysis adjusted for the individual differences in critical thinking skills upon admission and determined the strength of the relationship between critical thinking skills on admission to the program and gains found in critical thinking skills upon completion of the program. When significant statistical differences in the estimated marginal mean scores were found by the ANCOVA, polynomial contrasts were utilized to describe the pattern of those differences across cohorts. Patterns identified with this trend analysis determined whether the trend across cohorts appeared to be linear or curvilinear. Post hoc pairwise comparisons of the estimated marginal mean scores were performed to describe significant trends in more detail with no attempt to control overall Type 1 error rate. 


\section{Chapter Four: Results}

The purpose of this study was to determine the relationship between the fidelity and dose of human patient simulation (HPS), critical thinking abilities, and knowledge acquisition in an associate degree nursing program. In addition, this study examined the effectiveness of HPS when used as a teaching strategy in lieu of clinical experiences with actual patients. Data were collected from August 2004 through May 2010 for six cohorts of graduates who were exposed to varying degrees of fidelity and amount of simulation, as well as the amount of time HPS was substituted for actual clinical experiences.

Data were analyzed using SPSS 20.0 to answer the research question "How does the use of HPS impact critical thinking skills and knowledge of graduates of an associate degree nursing program?" This chapter contains the findings of the statistical analysis of the mean scores on the HESI $E^{2}$ and HESI $E^{2}$ CT scores to determine the impact of HPS on critical thinking skills and knowledge of the graduates.

\section{Analysis of Variance and Covariance}

To address each research question, one-way analysis of variance (ANOVA) was performed to determine if significant statistical mean differences were found in the HESI $\mathrm{E}^{2}$ scores and HESI E ${ }^{2}$ CT scores among the six cohorts. Statistical significance was set at the $p<$ .05. Polynomial contrasts were utilized to describe the pattern of those differences found across cohorts. Pairwise comparisons were done to describe in more detail the pattern of differences found with the polynomial contrasts without adjusting for potentially Type I error rate.

A univariate analysis of covariance (ANCOVA) was performed on the covariate of the critical thinking (CT) admission score to determine the strength of the relationship between the critical thinking skills of the six cohorts upon admission to the program and gains in the 
dependent variable (critical thinking skills) upon completion of the program. After adjusting for differences in the $\mathrm{CT}$ admission exam scores of the cohorts, estimated marginal means of the HESI $E^{2}$ CT scores were established for each of the six cohorts. Polynomial contrasts and pairwise comparisons among the HESI $\mathrm{E}^{2} \mathrm{CT}$ estimated marginal means with the presence of the covariant were performed to describe the pattern of differences found in critical thinking between cohorts without adjusting for potentially Type I error rate.

Tests for assumptions. Prior to conducting the ANCOVA, the homogeneity-ofregression (slope) assumption was tested. This test evaluates the interaction between the covariate (CT admission exam scores) and the factor (independent variable) in the prediction of the dependent variable (HESI $\mathrm{E}^{2} \mathrm{CT}$ exam scores). A significant interaction between the covariate (CT admission exam scores) and the factor (HPS) suggests that the differences on the dependent variable (HESI $\mathrm{E}^{2} \mathrm{CT}$ exam scores) among cohorts vary as a function of the covariate. If the interaction is significant, results from an ANCOVA are not meaningful and the ANCOVA should not be done (Field, 2009). The test for homogeneity-of-regression, depicted in Table 5, did not show a significant interaction between the CT admission exam scores and HPS at F(5, $187)=1.063, p=.382$; therefore, the ANCOVA was conducted to increase the power of any potentially significant differences found in critical thinking scores between the cohorts.

With the size of each cohort being determined by the number of graduates who had completed the nursing program within the prescribed two years, the size of the cohorts ranged from 22 to 45 . With the unequal group sizes, it was important to ensure that the assumptions of normality and homogeneity of variance were not violated when performing the ANOVA (Field, 2009). To determine if the HESI $E^{2}$ and HESI $E^{2}$ CT scores were normally distributed within each of the six cohorts, the Shapiro-Wilk test was performed. Normal distribution of scores for 
each cohort was confirmed at $p>.05$; therefore, no violations of the assumption of normality were identified. The $p$ values of the Shapiro-Wilk by cohort are presented in Table 8 .

Table 8 HESI $E^{2}$ and HESI $E^{2}$ CT Test of Normality by Cohorts

\begin{tabular}{|c|c|c|c|c|c|c|}
\hline \multirow[b]{2}{*}{ Cohort } & \multicolumn{3}{|c|}{ Shapiro-Wilk - HESI E ${ }^{2}$ CT } & \multicolumn{3}{|c|}{ Shapiro-Wilk - HESI E ${ }^{2}$} \\
\hline & Statistic & $\mathrm{df}$ & Sig. & Statistic & Df & Sig. \\
\hline 1 & .987 & 33 & .956 & .987 & 33 & .953 \\
\hline 2 & .960 & 39 & .172 & .959 & 39 & .162 \\
\hline 3 & .989 & 45 & .937 & .987 & 45 & .875 \\
\hline 4 & .974 & 22 & .799 & .966 & 22 & .624 \\
\hline 5 & .942 & 22 & .221 & .951 & 22 & .331 \\
\hline 6 & .951 & 26 & .246 & .956 & 26 & .312 \\
\hline
\end{tabular}

To determine if variances of the admission selection criteria, HESI $\mathrm{E}^{2}, \mathrm{HESI} \mathrm{E}^{2} \mathrm{CT}$, and admission CT exam scores in each cohort were equal, the Levene ${ }^{e e}$ s test for homogeneity of variance was performed. As depicted in Table 9, no significant differences in the variances of the admission GPA $(p=.447)$, ACT $(p=.303)$, HESI A2 scores $(p=.398)$, HESI E ${ }^{2}$ scores $(p=$ $.792)$, HESI $E^{2}$ CT scores $(p=.905)$, and CT exam scores $(p=.932)$ were noted among the cohorts; therefore, homogeneity of variances was assumed. 
Table 9 Test of Homogeneity of Variances Admission Criteria, HESI E ${ }^{2}$, HESI E ${ }^{2}$ CT, and CT

\begin{tabular}{lcccc}
\hline & Levene Statistic & $\mathrm{df1}$ & $\mathrm{df2}$ & Sig. \\
\hline Adm. GPA & .955 & 5 & 181 & .447 \\
ACT & 1.218 & 5 & 181 & .303 \\
HESI A $^{2}$ & 1.035 & 5 & 181 & .398 \\
E2 & .478 & 5 & 181 & .792 \\
E2CT & .312 & 5 & 181 & .905 \\
CT & .265 & 5 & 181 & .932 \\
\hline
\end{tabular}

HESI E $\mathbf{E}^{2}$. Descriptive statistics for HESI $E^{2}$ scores by cohort are presented in Table 10. Mean scores of the six cohorts ranged from $(M=813.9, S D=85.41)$ to $(M=900.15, S D=$ 95.25). Analysis of variance of the mean scores of the HESI $E^{2}$ between the six cohorts revealed a significant difference at $\mathrm{F}(5,181)=3.022, p=0.012$ in knowledge between the cohorts with an observed power of .857 .

Table 10 Descriptive Statistics for HESI $E^{2}$ Scores by Cohort

\begin{tabular}{ccc}
\hline Cohort & Mean & SD \\
\hline 1 & 832.61 & 104.76 \\
2 & 813.9 & 85.41 \\
3 & 838.73 & 103.7 \\
5 & 839.14 & 106.71 \\
6 & 875.91 & 91.12 \\
\hline
\end{tabular}


Polynomial contrasts. A significant, positive linear trend of the HESI $\mathrm{E}^{2}$ mean scores $\mathrm{F}(1,181)=11.677, p=.001$ as exposure to and number of traditional clinical hours substituted with simulation increased was the only polynomial contrast identified as being significant. The quadratic trend $\mathrm{F}(1,181)=2.371, p=.125$ and the cubic trend $\mathrm{F}(1,181)=.188, p=.665$ contrasts were found to be non-significant. Trend analysis of the mean scores of the HESI E ${ }^{2}$ across cohorts is depicted in Figure 3.

Figure 3 Trend Analysis of HESI $E^{2}$ Mean Scores Across Cohorts Means Plots

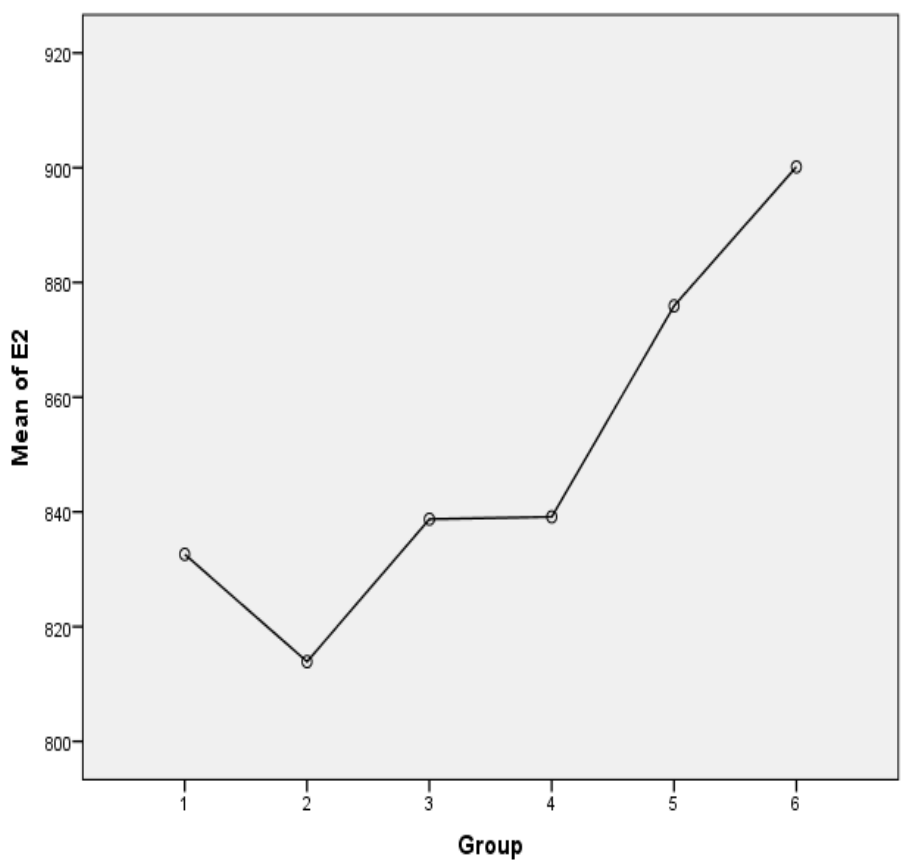

Pairwise comparisons. The significance of differences ( $p$ values) identified by pairwise comparisons of HESI $E^{2}$ mean scores between cohorts are presented in Table 11. Significant differences $(p<.05)$ in HESI $E^{2}$ mean scores were not found until comparisons were done with the two cohorts who had traditional clinical experiences substituted with HPS across all four semesters (cohorts 5 and 6). 
Table 11 Pairwise Comparisons of HESI $E^{2}$

\begin{tabular}{|c|c|c|c|c|c|}
\hline $\begin{array}{l}\text { Cohort } \\
\text { Contrasts }\end{array}$ & $\begin{array}{l}\text { Value of } \\
\text { contrast }\end{array}$ & Std. Error & $\mathrm{t}$ & $\mathrm{df}$ & $\begin{array}{l}\text { Sig. (2- } \\
\text { tailed) }\end{array}$ \\
\hline $1 \mathrm{v} 2$ & 18.71 & 23.203 & .806 & 181 & .421 \\
\hline $1 v 3$ & -6.13 & 22.483 & -.273 & 181 & .786 \\
\hline $1 \mathrm{v} 4$ & -6.53 & 27.001 & -.242 & 181 & .809 \\
\hline $1 \mathrm{v} 5$ & -43.30 & 27.001 & -1.604 & 181 & .111 \\
\hline $1 v 6$ & -67.55 & 25.725 & -2.626 & 181 & .009 \\
\hline $2 v 3$ & -24.84 & 21.462 & -1.157 & 181 & .249 \\
\hline $2 \mathrm{v} 4$ & -25.24 & 26.157 & -.965 & 181 & .336 \\
\hline $2 v 5$ & -62.01 & 26.157 & -2.371 & 181 & .019 \\
\hline $2 v 6$ & -86.26 & 24.837 & -3.473 & 181 & .001 \\
\hline $3 v 4$ & -.40 & 25.520 & -.016 & 181 & .987 \\
\hline $3 v 5$ & -37.18 & 25.520 & -1.457 & 181 & .147 \\
\hline $3 v 6$ & -61.42 & 24.166 & -2.542 & 181 & .012 \\
\hline $4 v 5$ & -36.77 & 29.578 & -1.243 & 181 & .215 \\
\hline $4 v 6$ & -61.02 & 28.418 & -2.147 & 181 & .033 \\
\hline $5 v 6$ & -24.24 & 28.418 & -.853 & 181 & .395 \\
\hline
\end{tabular}

As noted in Table 11, no significant differences in HESI $E^{2}$ mean scores were noted with the following cohort comparisons: $(1 * 2, p=.421),(1 * 3, p=.786),(1 * 4, p=.809),(1 * 5, p=$ $.111),(2 * 3, p=.249),(2 * 4, p=.336),(3 * 4, p=.987),(3 * 5, p=.147),(4 * 5, p=.215)$, and $(5 * 6$, $p=.395$ ). When compared to the control group with no exposure to HPS (cohort 1), nursing knowledge of graduates did not show significant improvement when traditional clinical hours 
were substituted with HPS for 10.5 - 13 hours in one semester (cohorts 2 and 3), 30 hours between two semesters (cohort 4 ), or 53 hours across four semesters of the program (cohort 5). However, significant differences in knowledge were noted with the cohorts exposed to HFS throughout all four semesters. Significant differences were found with the cohort comparisons of $(1 * 6, p=.009),(2 * 5, p=.019),(2 * 6, p=.001),(3 * 6, p=.012)$, and $(4 * 6$, $p=.033$ ). Cohort 5 (53 hours of substitution with HPS across all four semesters) demonstrated no significant increase in nursing knowledge, with the exception of cohort 2 (12 hours of substitution in one semester), when compared to the other cohorts. However, cohort 6 (57 hours of substitution with HPS across all four semesters) demonstrated a significant increase in knowledge when compared to cohorts 1,2,3, and 4. Although the HESI E ${ }^{2}$ mean score of cohort 6 (57 hours) was found to be higher than the mean score of cohort 5 (53 hours), the difference was not found to be significant.

Pairwise comparisons of the HESI $E^{2}$ mean scores revealed no significant differences between cohorts based on the fidelity of simulation exposure (cohorts 1-4). Mean scores of cohort 1 (control group with no HPS), cohort 3 (one semester of mixture of medium- and highfidelity HPS), and cohort 4 (three semesters of HPS) revealed a very slight positive linear trend, but mean differences were non-significant. Interestingly, the HESI E ${ }^{2}$ mean score of cohort 2 (one semester medium-fidelity HPS) was lower, although non-significant, than the mean score of cohort 1 (no HPS). However, significant increases in nursing knowledge in relation to fidelity were not identified until graduates were exposed to HPS in all four semesters. Significant positive increases in knowledge, measured by the HESI $\mathrm{E}^{2}$ mean scores, were noted with cohort 6 when compared with cohorts 1,2,3, and 4 . In addition, a significant increase in knowledge was found with cohort 5 when compared with cohort 2 . 
HESI E C $^{2}$. Descriptive statistics for the HESI $E^{2}$ CT scores by cohort are presented in Table 12. Mean scores of the six cohorts ranged from $(M=812.74, S D=86.53)$ to $(M=905.58$, $S D=100.73)$. Analysis of variance of the HESI $E^{2}$ CT mean scores between the six cohorts revealed a significant statistical difference at $\mathrm{F}(5,181)=3.740, p=0.003$ in critical thinking skills between the cohorts with an observed power of .929 .

Analysis of Covariance (ANCOVA). The critical thinking skills of students on admission to the program, as measured by the CT admission exam, revealed a significant, downward linear trend at $\mathrm{F}(1,181)=43.390, p<.001$ with each subsequent cohort admitted to the program. Cohort 1 had the highest CT mean score on admission to the program while cohort 6 had the lowest. After statistically adjusting for differences in the admission CT exam mean scores of the cohorts (covariate), estimated marginal means of the HESI $\mathrm{E}^{2} \mathrm{CT}$ exam scores were established for each of the six cohorts. The actual and estimated marginal means of the HESI $E^{2}$ CT exam scores are presented in Table 12.

Table 12 Descriptive Statistics for HESI E ${ }^{2}$ CT Scores and Covariate by Cohort

\begin{tabular}{cccccc}
\hline & \multicolumn{2}{c}{$\begin{array}{c}\text { HESI E } \\
\text { Actual Score }\end{array}$} & \multicolumn{2}{c}{ CT Ad Admission Exam } & HESI E ${ }^{2}$ CT Exam \\
Cohort & Mean & SD & Mean & SD & Mean \\
1 & 831.36 & 105.05 & 836.97 & 44.76 & 824.09 \\
2 & 812.74 & 86.53 & 811.03 & 53.50 & 809.91 \\
3 & 842.38 & 101.72 & 784.67 & 43.62 & 844.05 \\
4 & 840.32 & 106.54 & 785.91 & 41.02 & 841.78 \\
5 & 888.09 & 91.43 & 767.73 & 56.90 & 892.66 \\
6 & 905.58 & 100.73 & 762.31 & 57.08 & 911.07 \\
\hline
\end{tabular}


The mean score of the CT admission exam, the covariate, for the control group (cohort 1) was $836.97(\mathrm{SD}=44.76)$. The actual mean score of the HESI $\mathrm{E}^{2} \mathrm{CT}$ exam for the control group was $831.36(\mathrm{SD}=105.05)$ and the estimated marginal mean after ANCOVA analysis was 824.09. For cohort 6 (exposed to four semesters of HPS), the covariate mean score was 762.31 $(\mathrm{SD}=57.08)$, the actual mean score of the HESI $\mathrm{E}^{2} \mathrm{CT}$ exam was $905.58(\mathrm{SD}=100.73)$, and the estimated marginal mean was 911.07. As table 12 depicts, cohort 1 had a higher mean score on the $\mathrm{CT}$ admission exam than cohort 6 . Therefore, to compensate for this advantage, the estimated marginal mean of the HESI $\mathrm{E}^{2} \mathrm{CT}$ exam for cohort 1 is less than the actual mean score. Likewise, to compensate for the disadvantage of having a lower CT score on admission to the program, the estimated marginal mean for cohort 6 is greater than the actual HESI E ${ }^{2} \mathrm{CT}$ mean. Inclusion of admission CT scores as a covariate was not significant, $\mathrm{F}(1,180)=1.329, p=$ .250. As found with the ANOVA, analysis of covariance of the HESI $E^{2} \mathrm{CT}$ estimated marginal mean scores between the six cohorts revealed a significant statistical difference at $\mathrm{F}(5,180)=$ $3.958, p=.002$ with an observed power of .943 in critical thinking skills between the cohorts with the presence of the covariate (CT admission exam score).

Polynomial contrasts. The only significant polynomial contrast identified with the HESI $\mathrm{E}^{2} \mathrm{CT}$ mean scores and estimated marginal mean scores was a positive linear trend at $\mathrm{F}(1,181)=$ $14.836, p<.001$ as exposure to and the number of traditional clinical hours substituted with simulation increased. The quadratic trend at $\mathrm{F}(1,181)=2.144, p=.145$ and the cubic trend at $\mathrm{F}(1,181)=.381, p=.538$ were not found to be significant. It was interesting to note that cohort 6, who had the lowest admission CT mean score on admission, demonstrated the greatest gains in knowledge by having the highest HESI $E^{2} \mathrm{CT}$ mean score on exit. Linear trend analysis of the 
actual and estimated marginal means of the HESI $E^{2} \mathrm{CT}$ exam scores across cohorts compared to the linear trend analysis of the CT exam given on admission is depicted in Figure 4.

Figure 4 Trend Analysis of HESI $E^{2}$ CT Means and Admission CT Means Across Cohorts

\section{Means Plots}
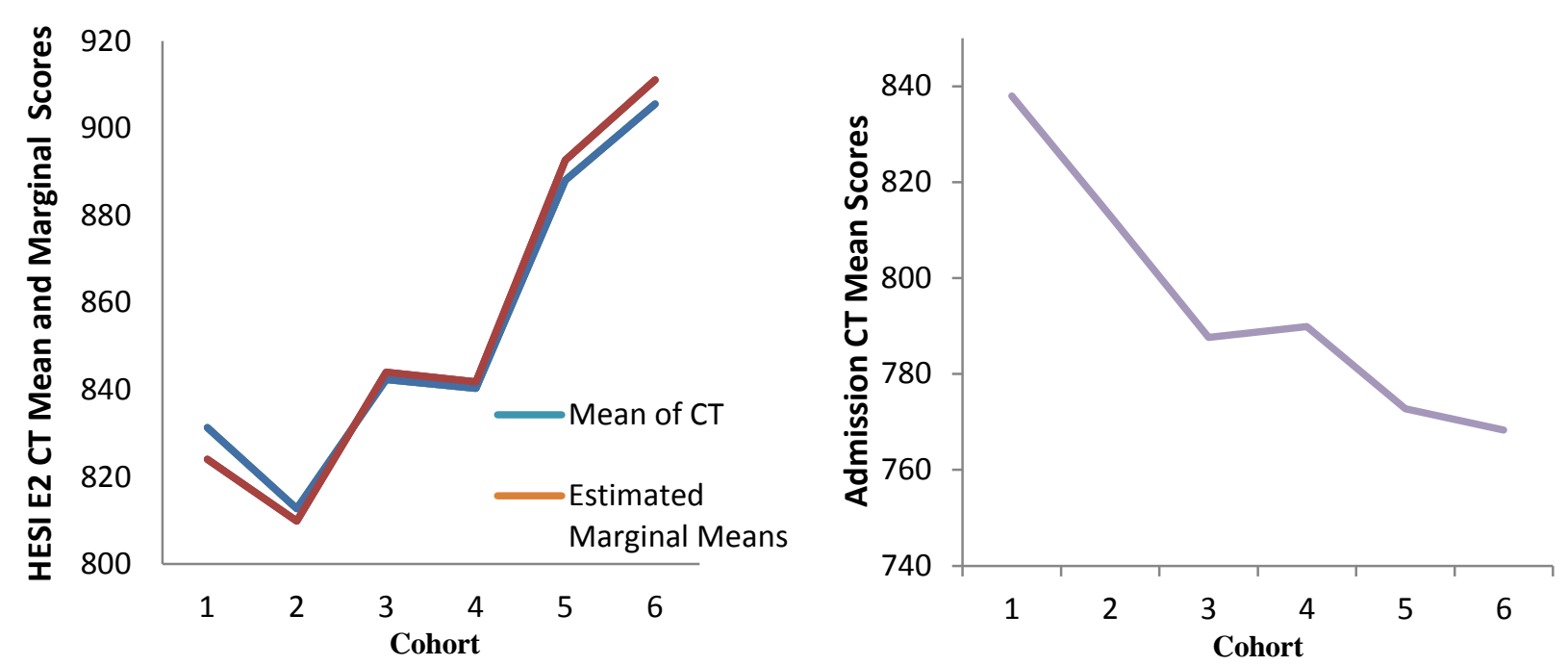

Pairwise comparisons. The significance of differences ( $p$ values) identified by pairwise comparisons of the HESI $\mathrm{E}^{2} \mathrm{CT}$ scores and estimated marginal mean scores are presented in Table 13. Although the $p$ values were different, pairwise comparisons identified the same significant differences $(p<.05)$ in both the actual and the estimated marginal means of the HESI $\mathrm{E}^{2} \mathrm{CT}$ scores between the following cohorts: $(1 * 5, p=.038 / .019),(1 * 6, p=.005 / .002),(2 * 5$, $p=.005 / .003),(2 * 6, p<.001 / .001),(3 * 6, p=.010 / .007)$, and $(4 * 6, p=.024 / .017)$.

Cohorts 5 (53 hours) and 6 (57 hours), who had the greatest number of hours of traditional clinical experiences substituted with HPS throughout all four semesters, demonstrated a significant increase in critical thinking when compared to cohort 1 (no HPS) and cohort 2 (12 hours of traditional clinical experiences substituted with HPS in one semester). In addition, 
Table 13 Pairwise Comparisons of HESI $E^{2} C T$

\begin{tabular}{lcccc}
\hline $\begin{array}{l}\text { Cohort } \\
\text { Comparisons }\end{array}$ & \multicolumn{2}{c}{$\begin{array}{c}\text { HESI E }{ }^{2} \text { CT Exam } \\
\text { Observed Mean }\end{array}$} & \multicolumn{2}{c}{$\begin{array}{c}\text { HESI E CT Exam } \\
\text { Adjusted Mean }\end{array}$} \\
\hline Value of contrast & Sig. (2-tailed) & Mean Difference &. Sig \\
\hline 1v3 & 18.62 & .426 & 14.18 & .549 \\
1v4 & -11.01 & .627 & -19.96 & .404 \\
$1 \mathrm{v} 5$ & -8.95 & .742 & -17.69 & .531 \\
$1 \mathrm{v} 6$ & -56.73 & .038 & -68.57 & .019 \\
$2 \mathrm{v} 3$ & -74.21 & .005 & -86.98 & .002 \\
$2 \mathrm{v} 4$ & -29.63 & .171 & -34.14 & .121 \\
$2 \mathrm{v} 5$ & -27.57 & .296 & -31.87 & .231 \\
$2 \mathrm{v} 6$ & -75.35 & .005 & -82.75 & .003 \\
$3 \mathrm{v} 4$ & -92.83 & .000 & -101.17 & .000 \\
$3 \mathrm{v} 5$ & 2.06 & .936 & 2.27 & .929 \\
$3 \mathrm{v} 6$ & -45.71 & .076 & -48.61 & .061 \\
$4 \mathrm{v} 5$ & -63.20 & .010 & -67.02 & .007 \\
$4 \mathrm{v} 6$ & -47.77 & .110 & -50.88 & .090 \\
$5 \mathrm{v} 6$ & -65.26 & .024 & -69.30 & .017 \\
\hline
\end{tabular}

cohort 6 , demonstrated a significant increase in critical thinking when compared to cohort 3 (10.5 - 13.5 hours of substitution in one semester) and cohort 4 (30 hours of substitution across two semesters). Although mean scores of the HESI E ${ }^{2} \mathrm{CT}$ exam for cohort 5 were higher than the mean scores for cohorts 3 and 4 , the differences were not significant. Comparison of cohorts 5 and 6 (exposed to HPS in all four semesters, but in differing amounts in the first semester) 
revealed that the mean score of the HESI $E^{2} \mathrm{CT}$ exam of cohort 6 (57 hours) was higher, although non-significant, than the mean score of cohort 5 (53 hours).

Pairwise comparisons of the mean scores and the estimated marginal means of the HESI $\mathrm{E}^{2} \mathrm{CT}$ exam revealed no significant differences in critical thinking between cohorts based on the fidelity of HPS used (cohorts 1, 2, 3, and 4). Interestingly, mean scores of the HESI E ${ }^{2} \mathrm{CT}$ exam were slightly lower for cohort 2 (one semester of medium-fidelity HPS) when compared to cohort 1 (no HPS) and for cohort 4 (three semesters of HPS) when compared to cohort 3 (one semester of a mixture of medium- and high-fidelity HPS).

Significant increases in critical thinking in relation to fidelity were not identified until graduates were exposed to HPS in all four semesters. Significant positive increases in both the actual and the estimated marginal mean scores of the HESI $E^{2} \mathrm{CT}$ exam were noted with the two cohorts exposed to four semesters of HPS (5 and 6) when compared with cohort 1 (no HPS) and cohort 2 (one semester of medium-fidelity HPS). In addition, a significant increase in critical thinking was found with cohort 6 when compared with cohort 3 (one semester of medium- and high-fidelity HPS) and cohort 4 (three semesters of HPS).

\section{Summary of Analysis}

Analysis of the results revealed that differences in the amount of exposure to and the number of hours of simulation that is substituted for traditional clinical experiences did have an impact on knowledge and critical thinking. A significant, positive linear trend with knowledge and critical thinking was identified across the six cohorts as exposure to and the number of traditional clinical hours substituted with simulation increased. Mean scores of both the HESI E ${ }^{2}$ and HESI $\mathrm{E}^{2} \mathrm{CT}$ were highest for the cohort with the greatest number of traditional clinical hours substituted with simulation. 
Only one statistical significant difference was found between the pairwise comparisons of the HESI $E^{2}$ and HESI $E^{2}$ CT exams. Mean scores of both the HESI $E^{2}$ and HESI $E^{2} \mathrm{CT}$ of cohort 6, exposed to 57 hours of HPS across the curriculum, were significantly higher than cohorts 1, 2, 3, and 4. Cohort 5, exposed to 53 hours of HPS across the curriculum, had a significant increase in the mean score of the HESI E ${ }^{2} \mathrm{CT}$ exam when compared to cohorts 1 and 2. However, the increase in the mean score of the HESI $E^{2}$ exam noted with cohort 5 was only found to be significant when compared to cohort 2 .

Fidelity of simulation was not found to have a significant impact on knowledge and critical thinking skills of students. Pairwise comparisons identified no significant differences in mean scores of the HESI $E^{2}$ and HESI $E^{2} \mathrm{CT}$ between the cohorts exposed to no HPS, to one semester of medium-fidelity HPS, one semester of a mixture of medium-fidelity and HPS, and to three semesters of HPS. However, it is important to point out the significant, positive linear trend found with cohorts 5 and 6 , which documented the positive impact on knowledge and critical thinking when HPS is integrated across the curriculum. But, it is equally important to point out that the amount of student exposure to HPS of cohorts 5 and 6 was much higher than the exposure of the other cohorts. Therefore, interpretations of the pairwise comparisons between cohorts 1,2,3, and 4 to draw conclusions about the impact of fidelity on knowledge and critical thinking are open to discussion.

Chapter Five includes a discussion and interpretation of the findings from the study. A comparison of the study"s findings with those discussed in the literature review of Chapter Two is included. The implications for nursing education are identified, as well as recommendations for further research. 


\section{Chapter Five: Summary, Conclusions, and Recommendations}

\section{Review of the Problem}

Nurse educators are being challenged to explore alternative approaches, which include innovative, evidenced based teaching pedagogies, to better prepare nurses to practice in todayes healthcare systems. Although nursing programs have used low-fidelity simulation in the form of task trainers and static mannequins for years to teach and evaluate psychomotor skills, a rapid growth in the use of human patient simulation (HPS) has been seen in the last few years. Research supporting the benefits and use of simulation is abundant; however, a paucity of research exists that directly evaluates the outcomes of HPS. As noted in literature reviews of simulation use in nursing, research studies varied in their designs and methods. Many studies had small sample sizes, lacked a control group, evaluated limited exposure to HPS, and lacked reliable and valid measurement tools designed to measure the learning outcomes of HPS. A plethora of research can be found that supports both student and faculty perceptions of satisfaction with, of the value of, and of the benefits of simulation, but these studies measured faculty and student responses to simulation with self-reports, as opposed to measuring actual student responses with objective measurement tools.

This study examined the impact of the integration of HPS on knowledge and critical thinking skills of students enrolled in an associate degree nursing program. Six cohorts of graduates exposed to varying types of fidelity and number of hours of simulation represented the sample. The HESI E ${ }^{2}$, a multiple choice exam with test items written at the application level and above, was the tool used to measure knowledge and critical thinking. This exam, given to all graduates at the completion of the program, has proven reliability and validity of predicting student success on the NCLEX-RN. Based on the NCLEX-RN test blueprint, the exam is representative of the knowledge and skills required of graduates for safe entry into nursing 
practice. All test items require multi-logical thinking to answer and are written at the higher cognitive levels of applying, analyzing, evaluating, and creating. The exam is designed to evaluate the student"s ability to think critically, which is necessary for effective decision making, problem solving, and forming clinical judgments within the context of nursing practice. In addition to providing a composite score that evaluates knowledge within the context of nursing practice, a critical thinking score is provided that measures the student"s ability to use critical thinking skills that are required for selecting the correct answer. These two scores, the HESI $E^{2}$ composite score and the HESI $\mathrm{E}^{2} \mathrm{CT}$ score, are used to measure knowledge and critical thinking skills of the graduates upon completion of the program.

Statistical analyses were performed to answer the following research question: How does the use of HPS impact critical thinking skills and knowledge of graduates of an associate degree nursing program? Specific questions were designed to answer this research question and to identify any differences in critical thinking skills and knowledge acquisition among cohorts exposed to different types of fidelity and number of hours of HPS. A summary of the findings of the study are discussed in relation to each research question. It is important to reiterate that, throughout this chapter, HPS refers to high-fidelity HPS unless otherwise noted.

\section{Summary of Findings}

Research Question 1. How does nursing knowledge, as determined by the HESI $E^{2}$ score, of graduating associate degree nursing students trained with high-fidelity HPS compare to those trained with medium-fidelity HPS or those not exposed to HPS?

Polynomial contrasts of the mean scores of the HESI E ${ }^{2}$ exam, the tool used to measure nursing knowledge, identified an upward linear trend across cohorts as exposure to fidelity of HPS increased. With the exception of cohort 2, who had a slight decrease in the mean score 
when compared to cohort 1 , increases in the mean scores of the HESI $E^{2}$ were identified as students were exposed to increased fidelity of HPS. However, pairwise comparisons revealed that this upward linear trend in knowledge acquisition was not found to be significant until cohorts were exposed to HPS across all four semesters of the curriculum. When comparing cohorts exposed to approximately the same number of hours of medium-fidelity or high-fidelity simulation with the control group exposed only to low-fidelity simulation, fidelity of simulation was not found to have a significant impact on knowledge acquisition. No significant differences in knowledge gain were identified when graduates exposed to either 12 hours in one semester of medium-fidelity HPS (cohort 2), 10.5 - 13 hours in one semester of a mixture of medium- and high-fidelity HPS (cohort 3), or 34 hours of HPS over three semesters (cohort 4) were compared to graduates exposed only to low-fidelity simulation (cohort 1). Therefore, nursing knowledge, measured by the HESI $E^{2}$ score, of graduating associate degree nursing students trained with HPS was found to be equivalent to those trained with medium-fidelity HPS or those not exposed to HPS. These findings did not support the hypothesis:

- A significant increase will be seen with knowledge gain, measured by the HESI $\mathrm{E}^{2}$ score, as cohorts are exposed to an increased fidelity of HPS.

Although graduates exposed to one semester of medium-fidelity HPS (cohort 2) had a lower HESI $E^{2}$ mean score than graduates with no exposure to HPS (cohort 1), the difference was non-significant and could have been a result of individual student or faculty variables not controlled in the study. It is important to note that these graduates were the first to experience simulated experiences with the use of medium-fidelity HPS in our program. Therefore, faculty skill and confidence may have impacted results. In addition, the method of delivery of HPS experiences for these graduates varied from that provided for other graduates. This cohort of 
graduates completed all HPS experiences in two days during one week of the final semester of the program. Experiences with HPS for all other graduates occurred in either one- or two-hour sessions spaced throughout the semester(s).

The findings of this study support that the effectiveness of the use of low-, medium-, and high-fidelity HPS are equivalent in achieving the outcome of knowledge acquisition. This finding is similar to other studies that compared the effectiveness of differences in the fidelity of simulation on knowledge. Jeffries and Rizzolo (2006) found no significant differences in knowledge gains when HPS was compared to a paper/pencil case study simulation and lowfidelity static mannequin simulation. Two studies by Kardong-Edgren et al. $(2007,2009)$ found no significant differences in knowledge gain when comparing the lecture format with low-, medium-, and high-fidelity HPS. Although Kardong-Edgren et al. (2007) identified increases in knowledge gain with the two simulation groups as opposed to the lecture group, the differences were not significant. In another study, Lapkin and Levett-Jones (2011) found no significant difference in knowledge acquisition when comparing medium-fidelity simulation and HPS. However, Shepherd et al. (2007) did find a significant gain in student scores on a systematic patient assessment with medium-fidelity HPS when compared to the traditional lecture format delivery and a scenario-based PowerPoint workshop.

Although this study did support previous research documenting equivalency with the effectiveness of medium-fidelity HPS and HPS on improving knowledge, it is important to point out the differences in the amount of simulation exposure of the graduates in this study. When comparing medium-fidelity HPS to a mixture of medium- and high-fidelity HPS, student exposure was similar, ranging from $10.5-13$ hours of exposure during one semester. When comparing these two cohorts with the cohort who was exposed to three semesters of HPS, 
student exposure was not equivalent. However, with the lack of a non-significant difference in the HESI $E^{2}$ mean scores of these three groups, one can conclude that the addition of HPS did not impact knowledge acquisition.

When comparing the three cohorts of graduates exposed to HPS (cohorts 4, 5, and 6) with those exposed to medium-fidelity HPS, a significant, positive linear trend in HESI $E^{2}$ mean scores was identified. As noted in Figure 2, mean scores of each cohort increased, with the exception of cohort 2, as exposure to HPS increased. Graduates exposed to 57 hours of HPS (cohort 6) and to 53 hours of HPS (cohort 5) across all four semesters had the highest HESI E ${ }^{2}$ mean scores respectively. Significant increases in knowledge, measured by the HESI E ${ }^{2}$ mean score, were identified when graduates exposed to 57 hours of HPS across four semesters were compared to graduates with no exposure to HPS, one semester of medium-fidelity HPS, one semester of a mixture of medium- and high-fidelity HPS, and 34 hours of HPS across three semesters. In addition, significant increases in knowledge were identified when graduates exposed to 53 hours of HPS over four semesters were compared to graduates exposed to 12 hours of medium-fidelity HPS. Although an increase in knowledge was identified with graduates exposed to 34 hours of HPS over three semesters when compared to graduates exposed to a semester of medium-fidelity, a semester of a mixture of medium- and high-fidelity, or no HPS, the increase was non-significant. This upward linear trend that was identified with significant increases when HPS was integrated across the curriculum did document that highfidelity simulation had a significant, positive impact on knowledge acquisition. However, with the increased number of hours of student exposure to HPS that were required to significantly impact knowledge acquisition when compared to graduates exposed to medium-fidelity HPS or a mixture of medium- and high-fidelity HPS, one can conclude that the combination of both high- 
fidelity and extended exposure led to the results found. With the limited experiences of the cohorts exposed to medium-fidelity simulation of this study, it is not possible to determine the impact extended exposure with medium-fidelity simulation may have on knowledge acquisition or if the impact of extended exposure with medium-fidelity simulation would yield the same results as extended exposure with HPS.

Research Question 2. Is there a difference in knowledge, as determined by the HESI $E^{2}$ score, among the cohorts of graduating associate degree nursing students based on the number of hours traditional clinical experiences were substituted with HPS?

Findings of this study did identify a difference in knowledge of the graduates of an associate degree nursing program based on the number of hours traditional clinical experiences were substituted with HPS. Polynomial contrasts identified a significant, positive linear trend in HESI $E^{2}$ mean scores of the cohorts, the tool used to measure knowledge, as the number of hours that traditional clinical experiences were substituted with HPS increased. This trend pattern identified by the polynomial contrasts supports the hypothesis:

- A significant, positive linear trend will be seen with knowledge gain, measured by the HESI $E^{2}$ score, as cohorts are exposed to an increased number of hours that HPS is substituted for traditional clinical experiences.

With the exception of graduates exposed to 12 hours of traditional clinical experiences substituted with medium-fidelity simulation during one semester of the program (cohort 2), HESI $E^{2}$ mean scores increased with each cohort of graduates as exposure to and the number of traditional clinical hours substituted with HPS increased. Cohort 2 were the only graduates noted as having a decrease in knowledge, although non-significant, when compared with graduates with no exposure to HPS (cohort 1). However, graduates exposed to HPS across all 
four semesters (cohorts 5 and 6) had a significant gain in knowledge when compared to these graduates.

Although an upward linear trend identified positive increases in knowledge as exposure to and the number of hours of traditional clinical experiences substituted with HPS increased, the increase in knowledge was found to be significant when traditional clinical hours were substituted with HPS across all four semesters of the program. A comparison of the two cohorts of graduates exposed to clinical hours substituted with HPS across all four semesters of the program revealed a higher HESI $E^{2}$ mean score, although non-significant, for graduates exposed to 57 hours (cohort 6) when compared to graduates exposed to 53 hours (cohort 5). Pairwise comparisons identified a significant increase in knowledge with graduates of cohort 6 when compared to graduates with no exposure to HPS (cohort 1), graduates with 10.5 - 13 hours of traditional clinical substitution during one semester (cohorts 2 and 3), and graduates with 30 hours of traditional clinical substitution over two semesters plus four additional hours during campus lab in one semester (cohort 4). However, the increase in knowledge identified with cohort 5, exposed to 53 hours as opposed to 57 hours across the four semesters, when compared to cohorts 1,2,3, and 4, was significant only when compared to cohort 2 .

Findings of this study did support that HPS has a positive impact on knowledge acquisition and are similar to other studies that evaluated the effectiveness of HPS on the acquisition of knowledge. Although exposure to HPS was limited to a single scenario, a day, or a semester of HPS and no control group was used to compare the use of HPS with other teaching strategies, Lewis and Ciak (2011), Burns et al. (2010), Elfrink et al. (2010), and Hoffmann et al. (2007) found significant gains in knowledge after students were exposed to HPS. Contrasting results were identified in two studies comparing the effectiveness of HPS with the traditional 
lecture or case study seminar. Brannan et al. (2008) found a significant increase in cognitive skills when students participated in a two-hour lab with HPS as opposed to a two-hour lecture. However, when comparing the addition of either a one-hour case study seminar or a scenariobased HPS with a didactic presentation, Scherer et al. (2007) found no improvement in knowledge with either intervention.

The findings of this study also documented that HPS simulated experiences are as effective as traditional clinical experiences on the acquisition of knowledge. Studies that compared the effectiveness of HPS and traditional clinical experiences were found to have findings similar to this study; however, the number of hours used for comparison was small. Beddingfield et al. (2011) compared the difference between traditional clinical experiences and HPS experiences for care of two selected types of patient conditions. No significant statistical correlations were found between scores on an exam and the type of clinical experiences (traditional patients versus HPS experiences) students were exposed to. HPS was found to be as effective as traditional clinical experiences for knowledge acquisition related to these two patient situations. Conflicting findings were found in a study by Hicks et al. (2009) that compared differences between groups exposed to 30 hours of traditional clinical experiences, 30 hours of equally mixed traditional clinical experiences and HPS, and 30 hours of HPS experiences during a two-week practicum in a critical care course. Significant decreases in posttest scores compared to pretest scores were found in all three groups. The group with traditional clinical hours retained the most knowledge $(88.5 \%)$ when compared with the group who had all 30 hours of traditional clinical substituted with HPS (82.9\%). However, the difference in knowledge retention was not found to be significant. 
Only one study was found that evaluated the impact of HPS on knowledge acquisition when used to replace a percentage of traditional clinical experiences across the curriculum. Sportsman et al. (2011) found no significant positive or negative differences in scores on a standardized exit exam for three cohorts of seniors exposed to differences in the percentage of time traditional clinical experiences were substituted with HPS. The authors concluded from these findings that HPS is as effective as traditional clinical experiences for knowledge acquisition.

In contrast to the study by Sportsman et al. (2011), this study identified a significant improvement in knowledge when HPS was substituted for 57 hours of traditional clinical experiences across all four semesters of the nursing program. Although graduates exposed to either 53 hours or 57 hours of traditional clinical hours substituted with HPS over four semesters had no significant differences in knowledge gain, it was only the graduates exposed to 57 hours of substitution who had significant greater gains in knowledge when compared to the other graduates of the study. With the exception of those student and faculty variables unable to be controlled, the only difference between these two cohorts of graduates who had clinical experiences substituted with HPS over all four semesters was the number of hours of substitution that occurred in the first semester of the program. Graduates exposed to 57 hours of substitution were exposed to two additional two-hour simulated clinical experiences (four hours of substituted traditional clinical experiences) in the first semester of the program. The significant impact on knowledge that was identified with graduates exposed to these four additional hours of HPS was not only interesting but surprising and will be discussed further with the summary of the findings. 
Research Question 3. How do the critical thinking skills, as determined by the Health Education Systems, Inc. Exit exam (HESI $\left.E^{2}\right)$ Critical Thinking (CT) score, of graduating associate degree nursing students trained with high-fidelity HPS compare to those trained with medium-fidelity HPS or those not exposed to HPS?

Polynomial contrasts of the mean scores of the HESI E ${ }^{2} \mathrm{CT}$ exam, the tool used to measure critical thinking, identified an upward linear trend across cohorts as exposure to fidelity of HPS increased. With the exception of cohorts 2 and 4, who had slight decreases in the mean scores when compared to cohorts 1 and 3 respectively, increases in the mean scores of the HESI $\mathrm{E}^{2} \mathrm{CT}$ were identified as students were exposed to increased fidelity of HPS. However, pairwise comparisons revealed that this upward linear trend in critical thinking was not found to be significant until cohorts were exposed to HPS across all four semesters of the curriculum. When comparing cohorts exposed to approximately the same number of hours of medium-fidelity or high-fidelity simulation with the control group exposed only to low-fidelity simulation, fidelity of simulation was not found to have a significant impact on enhancing critical thinking skills. No significant differences in critical thinking scores were identified when graduates exposed to either 12 hours in one semester of medium-fidelity HPS (cohort 2), 10.5 13 hours in one semester of a mixture of medium- and high-fidelity HPS (cohort 3), or 34 hours of HPS over three semesters (cohort 4) were compared to graduates exposed only to low-fidelity simulation (cohort 1). Therefore, critical thinking, measured by the HESI E ${ }^{2} \mathrm{CT}$ score, of graduating associate degree nursing students trained with HPS was found to be equivalent to those trained with medium-fidelity HPS or those not exposed to HPS. These findings did not support the hypothesis: 
- A significant increase will be seen with critical thinking, measured by the HESI $\mathrm{E}^{2}$ CT score, as cohorts are exposed to an increased fidelity of HPS.

Also noted with the HESI $E^{2}$ mean score measuring acquisition of knowledge, graduates exposed to one semester of medium-fidelity HPS (cohort 2) had a lower HESI E ${ }^{2}$ CT mean and estimated marginal mean score when compared to graduates with no exposure to HPS. In addition, it was interesting to note that the HESI $\mathrm{E}^{2} \mathrm{CT}$ mean and marginal mean score of graduates exposed to 34 hours of HPS over three semesters of the program (cohort 4) were slightly lower (2 points) than the mean score of graduates exposed to $10.5-13.5$ hours of a mixture of medium- and high-fidelity HPS in one semester of the program (cohort 3). Although pairwise comparisons identified both of these differences as non-significant, these two cohorts of graduates did show a decrease in critical thinking skills.

While differences in critical thinking skills of graduates on admission to the program were controlled with the ANCOVA, it was impossible to control for all other variables that may have impacted the results of this study. The decreases in critical thinking of these two cohorts of graduates are most likely a result of individual student or faculty variables not controlled in the study or a result of differences in the method of delivery or placement of HPS. Although it was not surprising that graduates exposed to 12 hours of medium-fidelity HPS had a slightly lower HESI $E^{2} \mathrm{CT}$ mean score when compared to the control group with no exposure to HPS, it is more difficult to understand the slight decrease in the mean scores when graduates exposed to 34 hours of HPS in three semesters were compared to graduates exposed to $10.5-13.5$ hours of a mixture of medium- and high-fidelity during one semester.

The cohort of graduates exposed to 34 hours of HPS across three semesters had some of the highest academic abilities of the six cohorts. These graduates had the second highest GPA 
mean and the highest ACT and HESI $\mathrm{A}^{2}$ mean of the six cohorts on admission to the program. However, these graduates ranked third on the CT admission exam mean score when compared with the other five cohorts. In attempts to look back and evaluate variables that may have impacted this cohort of graduates, it was interesting to recognize that this cohort of graduates would have been the cohort of graduates most affected by the high turn-over of faculty. As a result of retirements and career changes, several new nursing instructors were hired during the two years that these graduates were enrolled in the program. Therefore, one can only wonder how much the inexperience in teaching of these new faculty members, new to the role of nurse educator, impacted the outcomes of this cohort of graduates.

The findings of this study support that the effectiveness of the use of low-, medium-, and high-fidelity HPS is equivalent in developing critical thinking skills. With the review of literature, only one study in nursing was found that compared the impact of medium-fidelity HPS and HPS on clinical reasoning skills. Although Lapkin and Levett-Jones (2011) found a significant increase $(p=.001)$ in clinical reasoning with the group exposed to HPS as opposed to the group exposed to medium-fidelity HPS, exposure was limited to a 20 minute simulated experience. In addition, clinical reasoning was measured via direct observation of student performance with the use of a checklist of behaviors and thinking processes. No studies were found that compared the fidelity of HPS and critical thinking skills across a nursing program curriculum.

Although this study did not support the findings of Lapkin and Levett-Jones (2011) who found that HPS was more effective than medium-fidelity HPS, it is important to point out the differences in the amount of simulation exposure of the graduates in this study. When comparing medium-fidelity HPS to a mixture of medium- and high-fidelity HPS, student 
exposure was similar, ranging from 10.5 - 13 hours of exposure during one semester. When comparing these two cohorts with the cohort who was exposed to three semesters of HPS, student exposure was not equivalent. Similar to the findings with knowledge acquisition, lack of a non-significant difference in the mean scores of the HESI $E^{2}$ CT exam of these three cohorts, one can conclude that the addition of HPS did not impact critical thinking of these graduates.

When comparing the three cohorts of graduates exposed to HPS (cohorts 4, 5, and 6) with those exposed to medium-fidelity HPS, a significant, positive linear trend in HESI $\mathrm{E}^{2}$ CT mean scores was identified. As noted in Figure 3, mean scores of each cohort increased, with the exception of cohort 4, as exposure to HPS increased. Graduates exposed to 57 hours of HPS (cohort 6) and to 53 hours of HPS (cohort 5) across all four semesters had the highest HESI E ${ }^{2}$ CT mean scores respectively. Significant, positive increases in critical thinking, measured by the HESI E ${ }^{2}$ CT mean score, were identified when graduates exposed to 57 hours (cohort 6) and 53 hours (cohort 5) of HPS across four semesters were compared to graduates with no exposure to HPS (cohort 1) and one semester of medium-fidelity HPS (cohort 2). In addition, significant increases in critical thinking were identified when graduates of cohort 6 were compared to graduates exposed to one semester of a mixture of medium- and high-fidelity HPS (cohort 3) and 34 hours of HPS across three semesters (cohort 4).

It was interesting to note that graduates exposed to 34 hours of HPS over three semesters (cohort 4) had a decrease in the HESI $E^{2}$ CT mean score when compared to graduates exposed to a semester of a mixture of medium- and high-fidelity simulation (cohort 3). Furthermore, it was interesting to note the difference found in critical thinking mean scores between cohorts 5 and 6 . Although the linear trend identified significant increases in critical thinking of both cohorts when compared to cohorts 1 and 2, the significant increase in critical thinking found with cohort 6, 
exposed to an additional four hours of HPS during the first semester of the program, when compared to cohort 3 and 4 does raise some interesting questions that need to be explored.

The upward linear trend that was identified with significant increases when HPS was integrated across the curriculum did document that high-fidelity simulation had a significant, positive impact on critical thinking. However, with the increased number of hours of student exposure to HPS that were required to significantly impact critical thinking when compared to graduates exposed to medium-fidelity HPS or a mixture of medium- and high-fidelity HPS, one can conclude that the combination of both high-fidelity and extended exposure led to the results found. Further studies comparing medium- and high-fidelity HPS when integrated across the curriculum in equal amounts of exposure are needed to further validate if the effectiveness of simulation is equivalent when compared to differences in fidelity.

Research Question 4. Is there a difference in critical thinking skills, as determined by the HESI $E^{2}$ CT score, among the cohorts of graduating associate degree nursing students based on the number of hours traditional clinical experiences were substituted with HPS?

Findings of this study did identify a difference in the critical thinking skills of graduates of an associate degree nursing program based on the number of hours that traditional clinical experiences were substituted with HPS. Although the mean scores of the CT admission exam for each cohort of graduates decreased with each subsequent cohort admitted, results of the ANCOVA compared the graduates HESI $E^{2} \mathrm{CT}$ estimated marginal mean scores after controlling for differences in the $\mathrm{CT}$ admission exam scores. Polynomial contrasts identified a significant, positive linear trend with both the HESI E ${ }^{2} \mathrm{CT}$ mean scores and estimated marginal mean scores of the cohorts, the tool used to measure critical thinking, as the number of hours that traditional 
clinical experiences were substituted with HPS increased. This trend pattern identified by the polynomial contrasts supports the hypothesis:

- A significant, positive linear trend will be seen with critical thinking, measured by the HESI $E^{2}$ CT score, as cohorts are exposed to an increased number of hours that HPS is substituted for traditional clinical experiences.

With the exception of graduates exposed to 12 hours of traditional clinical experiences substituted with medium-fidelity simulation during one semester of the program (cohort 2) and graduates exposed to 30 hours of substituted traditional clinical experiences with HPS across two semesters plus four hours substituted for campus lab hours (cohort 4), HESI E ${ }^{2}$ CT mean scores increased with each cohort of graduates as exposure to and the number of traditional clinical hours substituted with HPS increased. Cohorts 2 and 4 were the only graduates noted as having a decrease in critical thinking, although non-significant, when compared respectively with graduates with no exposure to HPS (cohort 1) and graduates exposed to one semester of 10.5 13.5 hours of substitution with a mixture of medium- and high-fidelity HPS (cohort 3). However, graduates exposed to HPS across all four semesters (cohorts 5 and 6) had a significant gain in knowledge when compared to these graduates.

Although an upward linear trend identified positive increases in critical thinking as exposure to and the number of hours of traditional clinical experiences substituted with HPS increased, the increase in critical thinking was found to be significant when traditional clinical hours were substituted with HPS across all four semesters of the program. A comparison of the two cohorts of graduates exposed to clinical hours substituted with HPS across all four semesters of the program revealed a higher HESI $E^{2} \mathrm{CT}$ mean score, although non-significant, for graduates exposed to 57 hours (cohort 6) when compared to graduates exposed to 53 hours 
(cohort 5). Pairwise comparisons identified a significant increase in critical thinking with graduates of cohorts 5 and 6 when compared to graduates with no exposure to HPS (cohort 1) and graduates with 12 hours of traditional clinical substitution with medium-fidelity HPS during one semester (cohort 2). In addition, a significant increase in critical thinking was noted with cohort 6 when compared to graduates with 10.5 - 13 hours of traditional clinical substitution during one semester (cohort 3) and graduates with 30 hours of traditional clinical substitution over two semesters plus four additional hours during campus lab in one semester (cohort 4). Again, it was interesting to find the significant improvement in critical thinking that occurred with cohort 6 when four additional hours of HPS were included in the first semester of the program.

No study was found that evaluated the impact of HPS on critical thinking skills when used to replace a percentage of traditional clinical experiences across the curriculum. However, studies comparing the effectiveness of HPS with other methods of instruction have revealed mixed findings. Ravert (2008) found no significant differences in critical thinking skills between groups exposed to five weeks of regular instruction, small group discussions, and HPS. In a similar study, Brown and Chronister (2009) found no significant differences in critical thinking skills between groups exposed to lecture and HPS. However, Schumacher (2004) found significant differences in pre- and post-test scores measuring critical thinking skills when groups were exposed to HPS or a combination of HPS and classroom. No significant difference in critical thinking skills were found with the group exposed to classroom instruction. These findings are similar to the findings of Howard (2007), who found significant differences in preand post-test scores measuring critical thinking skills with groups exposed to HPS but no significant differences with groups exposed to interactive case studies. 
This study was the first to examine the impact of HPS on critical thinking skills when substituted for traditional clinical hours across the curriculum of a nursing program. Findings of the study did support that HPS is as good as traditional clinical experiences on improving critical thinking skills. In addition, findings of this study are the first to suggest that the use of HPS, when substituted for traditional clinical experiences across the curriculum of a nursing program, can improve critical thinking skills. The significant, positive linear trend identified as the number of traditional clinical hours substituted with HPS increased across all semesters of the program support the importance of integrating HPS across the curricula of nursing programs.

\section{Conclusions}

Findings of this study did demonstrate positive increases, although not all significant, in the acquisition of nursing knowledge and development of critical thinking skills as exposure to HPS increased. These findings are supported by Kolb"s experiential learning theory and Brown, Collins, and Duguid"s situated cognition theory. Participation in HPS experiences required active involvement of students in the learning experience and provided opportunity for problem solving and reflection, all necessary for learning (Dewey, 1933; Kolb, 1984). Learning and acquisition of knowledge, the retention of content, and a deeper understanding of the significance of content in different contexts are enhanced when students learn by doing in an environment resembling the context in the subject matter to be learned (Ironside, 2005; Kolb, 1984; Schon, 1987). Experiences with HPS provided the setting for students to live situations that resembled real-life care of specific patients in a particular situation. Through this real-life learning experience offered by HPS, students were able to learn the conditions for applying knowledge, seeing the implications of knowledge, and structuring knowledge for future use. Thus, learning becomes more useful, relevant, and transferable (Brill, 2001). 
In addition to the importance of active involvement of the learner in the learning experience, active reflection of the learner"s experiences is necessary for learning and increasing knowledge (Kolb, 1984; Kuiper \& Pesut, 2004). Through active reflection, new knowledge is gained that can be used to understand and deal with situations that follow (Dewey, 1933). In addition, opportunity for students to actively practice critical thinking skills (Schank, 1990) and discuss and reflect upon patient care experiences has a positive influence on the development of critical thinking skills (Murphy, 2004; Sedlak, 1997). As student exposure to HPS experiences increased, opportunity to practice critical thinking skills and to draw upon knowledge learned from previous experiences increased. Debriefing that occurred after every HPS could add to gains in knowledge and critical thinking skills as students were provided the opportunity to actively reflect on their actions and performance and to discuss alternative approaches that could have resulted in better patient outcomes.

Repeated and successful experiences in practice contribute to learning and are important for the development of professional knowledge (Schon, 1983), critical thinking skills (Ironside, 1999), and clinical judgment (Benner, 2004). Furthermore, McPeck (1981) recognized that critical thinking is teachable through exercises or problem solving, but it requires both the cognitive and affective domains of a studentes learning within the context of a specific discipline. As students participated in HPS, they were able to draw upon the base of nursing knowledge learned from past simulation experiences. In addition, opportunity to further develop their critical thinking skills existed as they attempted to understand and figure out the current patient"s health status, identify patient problems, make decisions about patient care, and form clinical judgments to effectively respond to the patient"s situation presented in the simulated experience. 
The results of the pairwise comparisons identifying the cohorts of graduates who had significant increases in either knowledge or critical thinking did reveal some interesting findings. Although graduates exposed to 12 hours of medium-fidelity HPS during one semester had no significant increase in knowledge or critical thinking when compared to graduates with no exposure to HPS, this finding was not a surprise. As discussed earlier, these graduates were the first graduates to be exposed to HPS and the delivery of the simulated experiences occurred in a one-time large dose within a week, unlike the other cohorts exposed to smaller doses spaced throughout the semester.

It was surprising to learn that graduates exposed to 34 hours of HPS over three semesters revealed no significant increases in knowledge or critical thinking when compared to graduates with no exposure to HPS, graduates with 12 hours of medium-fidelity HPS, or graduates with 10.5 - 13.5 hours of a mixture of medium- and high-fidelity HPS. However, significant, positive linear trends identified with both knowledge and critical thinking when graduates were exposed to HPS over four semesters suggest that HPS is more effective when integrated across the curriculum as opposed to being used in limited courses or semesters.

As noted by Hicks (2001) and Oermann (1997), critical thinking is an unfolding process that develops over time through a variety of contextual experiences. Time for reflection and processing of information have a positive correlation on development of critical thinking skills (Benner, 2004). Graduates of this study who were exposed to HPS experiences every other week throughout all four semesters of the program were provided with the opportunity to participate in a variety of planned, selected situations of practice, as opposed to the randomized experiences offered with traditional clinical experiences. As Kuiper and Pesut (2004) noted, the type of clinical experiences may be more important to one"s ability to reflect and gain knowledge than 
the number of years of experience in practice. With delivery of the HPS experiences occurring every other week throughout the four semesters of the program, students were provided with the time for reflection and processing of information related to the contextual simulated experiences, important for development of critical thinking skills. Ensuring exposure of all students to planned, selected situations of practice that were coordinated with the presentation of theory content over the course of the program can offer some explanation of the significant differences found in critical thinking and knowledge gains of graduates exposed to HPS across all four semesters, as opposed to one or two semesters. The importance of achieving the best outcomes when integrating HPS throughout the curriculum was also identified by Issenberg et al. (2005). However, the differences in knowledge and critical thinking found with the two cohorts exposed to HPS across all four semesters of the program was the most interesting and surprising find of the study. Although it was not surprising to find that graduates exposed to either 53 hours or 57 hours of HPS over four semesters had no significant differences in knowledge or critical thinking, it was surprising to find differences in the significance of the increases in knowledge and critical thinking when these two cohorts were compared to other cohorts. Graduates exposed to 57 hours of HPS integrated across the curriculum had significant gains in knowledge and critical thinking when compared to all other graduates in the study. In contrast, graduates exposed to 53 hours of HPS integrated across the curriculum only had significant increases in knowledge when compared to the graduates with 12 hours of medium-fidelity HPS during one semester and increases in critical thinking when compared to graduates with no exposure to HPS and graduates with 12 hours of medium-fidelity HPS during one semester.

With the exception of those student and faculty variables that could not be controlled, the only difference between these two cohorts of graduates who had clinical experiences substituted 
with HPS across all four semesters was the number of hours of substitution that occurred in the first semester of the program. Graduates exposed to 57 hours of substitution were exposed to two additional two-hour simulated clinical experiences (four hours of substituted traditional clinical experiences) in the first semester of the program. These findings lead one to question why the addition of four hours of HPS during the first semester of the program had such a significant impact on knowledge acquisition and critical thinking. These findings do suggest that the amount of exposure to HPS and the placement of HPS in the curriculum are important factors that could impact the outcomes of HPS.

As noted in a study by White (2003), self-confidence is important in developing critical thinking skills and influencing clinical decision-making. Brown and Chronister (2009) revealed that higher self-confidence ratings from students were significantly related to higher critical thinking scores. Furthermore, White concluded that a better understanding of the patiente's situation occurs as students gained experience, self-confidence, and knowledge. Repetition and repeated practice with realistic clinical situations can increase a student ec $^{\text {s }}$ confidence and competency (Blum et al., 2010).

Inexperienced students can experience feelings of anxiety, fears of making mistakes, and decreases in confidence when placed in traditional clinical experiences with real-life patients. Both anxiety and a lack of confidence can interfere with a student's ability to acquire and retain new knowledge (Lundberg, 2008). The impact of four additional hours of simulation exposure on anxiety and self-confidence of inexperienced first semester students may provide one possible explanation for the significant difference found in this study. The significant increases in knowledge and critical thinking found with the graduates exposed to 57 hours of HPS, as 
opposed to 53 hours, may have been the result of the impact on knowledge retention and critical thinking development when anxiety is decreased and self-confidence is improved.

Schlairet and Pollock (2010) found similar results when comparing two weeks of traditional clinical experiences with two weeks of HPS clinical experiences. Although Schlairet and Pollock found no significant differences in knowledge scores of the traditional clinical group when compared to the simulated clinical group, a steeper positive incline from pretest to posttest scores was identified with the group who was exposed to simulation clinical experiences prior to traditional clinical experiences. These findings support that student exposure to simulation experiences early in a nursing program prior to traditional experiences may have a positive impact on student learning outcomes and provide another possible explanation for the significant differences found between the two cohorts exposed to HPS throughout all four semesters. The cohort exposed to 57 hours of HPS across the curriculum participated in HPS every two weeks; whereas, the cohort exposed to 53 hours participated in HPS every three weeks. What impact did the frequency of exposure to (every 2 weeks versus 3 weeks) and placement of the HPS experiences within the semester (participation in more HPS scenarios prior to starting clinical in the acute care setting) have on the increase in knowledge and critical thinking?

It is difficult to draw conclusions as to why the cohort with four additional hours of HPS had significant increases in knowledge and critical thinking. As noted earlier, possible explanations for the increase could be the result of an increase in knowledge retention and confidence that can occur with experiential learning and experience. Placement of the additional hours within the semester, as well as the content of the extra scenarios may have impacted the student"s knowledge and critical thinking. In addition, the simulation instructor gained confidence and skill with each year of experience. Therefore, her effectiveness in leading the 
debriefing session, one of the most important features of the simulation experience, may have had a significant impact on the gains in knowledge and critical thinking of the last cohort in the study.

\section{Recommendations}

Recommendations for Practice. The results of this study add support to the current literature of the value of HPS in nursing education. Findings demonstrated positive outcomes related to knowledge and critical thinking with the integration of HPS across the curriculum of an associate degree program. Using a tool with established reliability and validity for measuring knowledge and critical thinking specific to the discipline of nursing, a significant, positive linear trend was identified as student exposure to HPS and amount of HPS substituted for traditional clinical experiences increased. Only one other study was found in nursing education that used an objective, reliable tool specific to nursing to examine the impact of HPS on knowledge acquisition when used as a replacement for traditional clinical experiences across the curriculum. This study was the first to examine the impact of HPS on critical thinking skills, when integrated throughout the curriculum as a replacement for a small percentage of traditional clinical experiences. HPS experiences when used as a replacement for a percentage of traditional clinical hours were found to be equally effective in achieving the student learning outcomes of knowledge acquisition and critical thinking. Furthermore, the findings of this study identified greater gains in knowledge and critical thinking when HPS was integrated throughout the curriculum as a replacement for a small percentage of traditional clinical experiences, suggesting that HPS may be more effective than traditional clinical experiences.

It is not my intent to suggest that HPS can or should totally replace traditional clinical experiences. However, this finding does provide support that HPS can be used as a replacement 
for a percentage of traditional clinical hours and achieve the same or better outcomes related to knowledge acquisition and critical thinking. However, further research is needed to identify the most appropriate ratio of direct patient care hours and simulation hours, as well as the most appropriate placement and sequencing of HPS in the curriculum.

With the current model of nursing education placing students in health care systems to provide care for actual patients, nurse educators are presented with barriers that prohibit enrollment increases and deter attempts to improve the quality of nursing education. Supplementing student clinical experiences with HPS can provide a viable alternative to overcome many of the barriers imposed by traditional clinical experiences. By providing opportunity for all students to participate in planned scenarios of selected patient conditions with the use of HPS, nurse educators can control for inconsistencies in patient assignments and standardize student learning opportunities. By carefully planning the types of scenarios used, faculty can ensure that all students are exposed to a variety of patient situations and low incidence, highly critical events. In addition, classroom theory can be coordinated with simulated clinical experiences to enhance transfer of knowledge. Participation in HPS provides opportunities for students to be active participants in their learning; work collaboratively in teams; practice critical thinking skills, make decisions, and form clinical judgments in a safe setting without risk to patient safety; and perform actions and skills lost in the clinical setting as a result of practice boundaries or agency limitations.

This study documents that clinical experiences with HPS is as effective as traditional clinical experiences. In addition, when used to replace a percentage of traditional clinical hours across the curriculum of a nursing program, HPS can enhance the acquisition of nursing knowledge and critical thinking skills. These findings add to the current literature in providing 
evidence-based research for nurse educators and regulatory bodies as they struggle with decisions related to the role of HPS in clinical education.

- Nurse educators who struggle to find sufficient and quality clinical placement sites can be confident that decisions to replace a percentage of traditional clinical experiences with HPS will not negatively affect student outcomes. HPS can be equally effective in achieving student outcomes of knowledge and critical thinking and when integrated across the curriculum, HPS can have a positive impact on these outcomes.

- Boards of Nursing can take into consideration the findings of this study as they review and revise their education rules to include the role of HPS in the clinical education of nurses.

Although this study documents the value of integrating HPS across the curriculum of an associate degree nursing program, the fidelity of simulation was not found to have an impact on student learning outcomes. No significant differences in knowledge or critical thinking were identified when comparing graduates exposed only to the traditional low-fidelity mannequins for skill acquisition, to a semester of medium-fidelity HPS or mixture of medium- and high-fidelity HPS, and to three semesters of HPS. These findings have significant implications for nursing education as nurse educators and administrators attempt to justify the costs associated with HPS. If the use of medium-fidelity HPS is just as effective as HPS in achieving student learning outcomes, then the high costs associated with HPS would be hard to justify. Scarce resources spent on high-fidelity mannequins and the faculty training required for their use may serve a greater number of students if financial resources were used to purchase medium-fidelity mannequins. 
The findings of this study did support previous research that has documented the effectiveness of medium-fidelity HPS in achieving similar outcomes when compared with HPS. However, it is important to note that when comparing knowledge and critical thinking scores of graduates based on the type of HPS fidelity, graduates used for comparison in this study were not equal in their exposure to HPS. Findings of this study did include:

- Significant, positive linear trends identified with knowledge and critical thinking when graduates were exposed to HPS across all four semesters of the program.

- The cohort of graduates exposed to one week of concentrated simulation experiences had the lowest HESI $E^{2}$ and HESI $E^{2} \mathrm{CT}$ exam mean scores of all cohorts. The difference in how HPS was integrated with this cohort of graduates as compared to other cohorts may have impacted this outcome. This finding does suggest that condensing all HPS experiences into a short period of time may not be as effective as spreading HPS experiences over the course of a semester and/or program.

Recommendations for Further Research. Findings of this study hold promise in suggesting that the integration of HPS across all semesters of a program, in lieu of a certain percentage of traditional clinical hours, can have a positive impact on knowledge acquisition and critical thinking. Furthermore, these findings offer insight as to the best method of delivery for the integration of HPS into nursing curricula. In addition to the amount of student exposure to HPS, the effectiveness of HPS may be impacted by the placement of HPS experiences in the curriculum, as well as the method of delivery of HPS experiences.

As noted earlier, these findings do raise some interesting questions. Why did four additional hours of HPS experiences have a greater significant impact on knowledge acquisition and development of critical thinking skills? Were outcomes related to the additional opportunity 
for students to draw upon knowledge learned and practice critical thinking skills from exposure to additional planned selected patient experiences or critical events? If so, what is the amount of student exposure to HPS that is required to make a significant impact on the acquisition of knowledge and critical thinking? Or were outcomes related to the placement of the additional experiences or the content of the additional experiences? Is there a correlation between student learning outcomes and placement of HPS in the curriculum? Did exposure to HPS early in the program, prior to patient contact in the acute care setting, have a greater impact on knowledge and critical thinking when anxiety relating to the fear of harming real patients is decreased and student self-confidence is increased?

With the decreases noted in knowledge and critical thinking of the graduates who completed all simulated experiences during one week of the semester, one might wonder if the method of delivery of HPS is an important factor affecting outcomes. In addition, with the variations in fidelity and amount of exposure to HPS of cohorts in this study, it was difficult to compare the effectiveness of medium- and high-fidelity HPS. Further research is needed to determine if medium-fidelity HPS would be just as effective as HPS when medium-fidelity HPS experiences are integrated throughout the curriculum of a nursing program.

Further studies are needed that examine the impact of HPS when integrated across curricula of nursing programs. Replication of this study, controlling for differences in the variables of fidelity, the amount of exposure, method of delivery, and placement within the curriculum of HPS would add valuable information to the current nursing research. In addition, adding a qualitative piece to studies can capture students" thoughts and comments during and after the simulation and debriefing sessions. Data obtained from reviewing videotapes of the 
simulation experience and/or student focused groups can provide further insight into and support of the quantitative findings. Further studies are needed to examine:

- The impact on outcomes of knowledge and critical thinking in relation to the percentage of traditional clinical hours substituted with HPS across the curricula of nursing programs. Determining the best mixture of traditional clinical hours and simulated clinical hours is needed to provide nurse educators and professional regulatory bodies with evidence-based guidelines for decision making and curricula improvements in nursing education.

- The best method of delivery for integration of HPS experiences in nursing curricula. Comparing differences in student learning outcomes based on the method of delivery is needed to determine the best method for achieving student learning outcomes. Is HPS more effective in achieving outcomes when students are exposed more frequently to smaller doses of HPS or larger doses that occur less frequently?

- The most appropriate placement for HPS in the curriculum. Studies comparing differences in the placement of HPS in the curriculum and the impact on student learning outcomes are needed to provide nurse educators with evidence-based guidelines for curriculum development and revisions. Are there significant differences in knowledge and critical thinking based on when students are exposed to HPS? Is HPS more effective in achieving outcomes if student exposure is increased early in the program, occurs more frequently towards the end of the program, or is equally distributed throughout the program?

- The effectiveness of medium- and high-fidelity simulation when integrated throughout all semesters of the program. Comparing cohorts with equal exposure to 
medium- and high-fidelity simulation would be a valuable contribution to the literature to better determine if medium-fidelity HPS is just as effective as HPS on acquisition of knowledge and development of critical thinking skills.

- The impact of the use of HPS during nursing education programs on the confidence, skills, and knowledge of graduates in their first job assignment. Does HPS ease the transition from education to practice?

\section{Limitations}

It is important to reiterate that findings from this study may not have been a direct result of HPS. Although this study did reveal some interesting findings, being an ex post facto study did limit the assumptions that could be drawn. The integration of HPS into the curriculum of the associate degree nursing program was done without the design of a study in mind. With the study spanning the years 2004 through 2010, it was not possible to control for all variables that may have had an impact on the knowledge and critical thinking of graduates. Although descriptive statistics of student demographics and academic abilities revealed cohorts were similar on admission, it was not possible to control for all student variables such as prior experiences in healthcare, opportunities provided with clinical experiences, and differences in clinical faculty teaching abilities, as well as outside stressors and factors that may have impacted a student"s ability to learn.

Attempts to control faculty variables were difficult as well. During the time frame of the study, primarily between the years of 2006 to 2008 , a large turnover of faculty occurred. The majority of nursing instructors hired were new to the role of nurse educator. This turnover of faculty may have impacted the presentation of theory content in the classroom as the more experienced faculty retired and the novice faculty began to gain confidence in their method of 
classroom delivery of content. As noted earlier, differences in faculty confidence and experience with the presentation of theory content may have impacted the students"e acquisition of knowledge and development of critical thinking. This variable may offer some explanation as to findings related to the cohort of graduates exposed to 34 hours of HPS over three semesters.

Faculty variables related to the integration of HPS were easier to control. The faculty member who initiated the use of HPS was assigned to the role of simulation coordinator. In this role, she conducted all HPS labs and debriefing sessions, which provided for consistency in conducting HPS experiences. However, her knowledge of and confidence with conducting simulations and leading debriefing sessions most likely increased over the years. This increase in her effectiveness in conducting HPS experiences may account for some of the significant differences found in knowledge acquisition and critical thinking with the last cohort of graduates.

In addition, HPS was integrated into the curriculum as opportunities arose with purchases of equipment and faculty resources. HPS was not integrated with the intent of conducting a study. Cohorts were pre-set, and were determined by the success of the students in completing the program. As a result, cohort numbers were small and unequal.

In addition to the variables examined in the study (fidelity of HPS and amount of exposure to and the number of hours of substitution with HPS), differences did exist in the method of delivery of HPS and placement of HPS within the curriculum. These variations within the cohorts of graduates made it difficult to draw conclusions from the findings related to the impact of fidelity on knowledge and critical thinking. In addition to differences in simulation fidelity, variations in the method of delivery, the number of hours of exposure, and placement within the curriculum occurred between the cohorts of graduates. It was difficult to determine if 
just one of these variables impacted the findings or if a combination of variables influenced the findings.

\section{Ending Thoughts}

As a result of this study, the effectiveness of the use of HPS as a supplement or alternative to traditional clinical experiences in an associate degree nursing program is clearly documented. Gains in both nursing knowledge and critical thinking skills demonstrated a positive, linear trend as student exposure to and the number of hours traditional clinical experiences were substituted with HPS increased. These findings suggest that HPS is as effective as traditional clinical experiences in achieving the student learning outcomes related to knowledge and critical thinking. Significant gains were noted when traditional clinical experiences were substituted with HPS across the curriculum of the program. This finding suggests that the integration of HPS as a substitution for a small percentage of traditional clinical hours was more effective on increasing knowledge and critical thinking skills when integrated across the curriculum. However, further research is needed to determine what percentage of substitution provides the best mixture of traditional and simulated clinical experiences for optimal achievement of student outcomes.

The findings of this study do not support that HPS is any more effective than mediumfidelity HPS in achieving the student outcomes of knowledge and critical thinking. However, variations of the amount of exposure, method of delivery, and placement within the curriculum made it difficult to compare fidelity. Further studies comparing medium-fidelity simulation and HPS when integrated across the curricula of nursing programs would provide stronger comparisons for determining if medium-fidelity HPS is as effective as HPS on achieving student outcomes. 


\section{References}

Abdo, A., \& Ravert, P. (2006).Student satisfaction with simulation experiences. Clinical Simulation in Nursing Education, 2(1), e13-e16. Doi:10.1016/j.ecns.2009.05.009

Ackermann, A. D. (2009). Investigation of learning outcomes for the acquisition and retention of CPR knowledge and skills learned with the use of high fidelity simulation. Clinical Simulation in Nursing, 5(6), e213-e222. Doi:10.1016/j.ecns.2009.05.002

Adamson, K. (2010). Integrating human patient simulation into associate degree nursing curricula faculty experiences, barriers, and facilitators. Clinical Simulation in Nursing,6(3), e75-e81. Doi:10.1016/j.ecns.2009.06.002

Akhtar-Danesh, N., Baxter, P., Valaitis, R. K., Stanyon, W., \& Sproul, S. (2009).Nurse faculty perceptions of simulation use in nursing education. Western Journal of Nursing Research, 31(3), 312-329. Doi:10.1177/0193945908328264

Alfaro-LeFevre, R. (1995). Critical thinking in nursing: A practical approach. Philadelphia, PA: W.B. Saunders.

Alfaro-LeFevre, R. (2009). Critical thinking and clinical judgment: A practical approach to outcome-focused thinking ( $4^{\text {th }} \mathrm{ed}$.). St. Louis, Mo: Saunders.

Alinier, G., Hunt, W., \& Gordon, R. (2004). Determining the value of simulation in nurse education: study design and initial results. Nurse Education in Practice, 4, 200-207. Doi:10.1016/S1471-5953(03)00066-0

Alinier G., Hunt B., Gordon R., \& Harwood, C. (2006). Effectiveness of intermediate-fidelity simulation training technology in undergraduate nursing education. Journal of Advanced Nursing, 54(3), 359-369.

American Association of Colleges of Nursing. (2008).The essentials of baccalaureate education for professional nursing practice. Retrieved from http://www.aacn.nche.edu/educationresources/BaccEssentials08.pdf

American Association of Colleges of Nursing. (2011). Shaping the future of nursing education [2011 Annual Report]. Retrieved from http://www.aacn.nche.edu/aacnpublications/annual-reports/AR2011.pdf

American Nurses Association. (2004). Scope and standards of practice. Silver Spring, MD: Nursesbooks.org

Angel, B. F., Duffey, M., \& Belyea, M. (2000). An evidence-based project for evaluating strategies to improve knowledge acquisition and critical-thinking performance in nursing students. Journal of Nursing Education, 39(5), 219-228. 
Ard, N., Rogers, K., \& Vinten, S. (2008). Summary of the survey on clinical education in nursing [Headlines from the NLN]. Nursing Education Perspectives, 29(4), 238-245.

Baker, C., Pulling, C., McGraw, R., Dagnone, J. D., Hopkins-Rosseel, D., \& Medves, J. (2008). Simulation in interprofessional education for patient-centered collaborative care. Journal of Advanced Nursing, 64(4), 372-379. Doi:10.1111/j.1365-2648.2008.04798.x

Bambini, D., Washburn, J., \& Perkins, R. (2009). Outcomes of clinical simulation for novice nursing students: Communication, confidence, clinical judgment. Nursing Education Perspectives, 30(2), 79-82.

Bearnson, C. S., \& Wiker, K. M. (2005). Human patient simulators: A new face in baccalaureate nursing education at Brigham Young University. Journal of Nursing Education, 44(9), 421-425.

Beddingfield, S., Davis, B. W., Gilmore, M., \& Jenkins, L. (2011).The effect of high-fidelity simulation on examination performance. Teaching and Learning, 6, 46-49. Doi: 10.1016/j.teln.2010.10.001

Benner, P. (2000). The wisdom of our practice: Thoughts on the art and intangibility of caring practice. American Journal of Nursing, 100(10), 99-105.

Benner, P. (2004). Using the Dreyfus Model of Skill Acquisition to describe and interpret skill acquisition and clinical judgment in nursing practice and education. Bulletin of Science, Technology \& Society, 24(3), 188-199.doi: 10.1177/0270467604265061

Benner, P., Sutphen, M., Leonard, V., \& Day, L. (2010).Educating nurses: A call for radical transformation. San Francisco, CA: Jossey-Bass.

Berndt, J. (2010). The ethics of simulated nursing clinical experiences. Teaching and Learning in Nursing, 5, 160-163. Doi:10.1016/j.teln.2010.03.004

Beyea, S. C., \& Kobokovich, L. J. (2004). Human patient simulation: A teaching strategy. AORN Journal, 80(4), 738-42.

Beyer, B. K. (1987).Practical strategies for the teaching of thinking. Boston, MA: Allyn and Bacon.

Bigge, M., \& Shermis, S. (2004). Learning theories for teachers. Boston, MA: Pearson Education.

Billings, D. M., \& Halstead, J. A. (2009).Teaching in nursing: A guide for faculty (3 ${ }^{\text {rd }}$ ed.). St. Louis, MO: Saunders/Elsevier.

Bjork, I. T., \& Kirkevold, M. (1999). Issues in nurses ${ }^{e e}$ practical skill development in the clinical setting. Journal of Nursing Care Quality, 14(1), 72-84. 
Blum, C., Borglund, S.,\& Parcells, D. (2010). High-fidelity nursing simulation: Impact on student self-confidence and clinical competence. International Journal of Nursing Education Scholarship, 7(1), Article 18. Doi:10.2202/1548-923X.2035

Boland, D. L. (2009).Developing curriculum: Frameworks, outcomes, and competencies. In D. M. Billings \& J. A. Halstead (Eds.), Teaching in nursing: A guide for faculty $\left(3^{\text {rd }}\right.$ ed., pp. 137-153). St. Louis, MO: Saunders/Elsevier.

Bradley, P. (2006). The history of simulation in medical education and possible future directions. Medical Education, 40, 254-262. Doi:10.1111/j.1365-2929.2006.02394.x

Brammer, J. D. (2006). RN as gatekeeper: Student understanding of the RN buddy role in clinical practice experience. Nurse Education in Practice, 6(6), 389-396. doi.org/10.1016/j.bbr.2011.03.031

Brannan, J. D., White, A., \& Bezanson, J. L. (2008). Simulator effects on cognitive skills and confidence levels. Journal of Nursing Education, 47(11), 495-500.

Bray, B., Schwartz, C. R., Weeks, D. L., \& Kardong-Edgren, S. (2009). Human patient simulation technology: Perceptions from a multidisciplinary sample of health care educators. Clinical Simulation in Nursing, 5(4), e145-e150.

Doi:10.1016/j.ecns.2009.02.002

Bremner, M. N., Aduddell, K., Bennett, D. N., \& VanGeest, J. B. (2006). The use of human patient simulators: Best practices with novice nursing students. Nurse Educator, 31(4), 170-174.

Brewer, E. P. (2011). Successful techniques for using human patient simulation in nursing education. Journal of Nursing Scholarship, 43(3), 311-317. Doi:10.1111/j.15475069.2011.01405.x

Brill, J. M. (2001). Situated cognition. In M. Orey (Ed.), Emerging perspectives on learning, teaching, and technology. Retrieved from http://projects.coe.uga.edu/epltt/

Brooks, K. L., \& Shepherd, J. M. (1990). The relationship between clinical decision -making skills in nursing and general critical thinking abilities of senior nursing students in four types of nursing programs. Journal of Nursing Education, 29(9), 391-399.

Broussard, L., Myers, R., \& Lemoine, J. (2009). Preparing pediatric nurses: The role of simulation-based learning. Issues in Comprehensive Pediatric Nursing, 32, 4-15. Doi:10.1080/01460860802610178

Brown, D., \& Chronister, C. (2009).The effect of simulation learning on critical thinking and self-confidence when incorporated into an electrocardiogram nursing course. Clinical Simulation in Nursing, 5(1), e45-e52. Doi:10.1016/j.ecns.2008.11.001 
Bruce, S. A., Scherer, Y. K., Curran, C. C., Urschel, D. M., Erdley, S., \& Ball, L. S. (2009). A collaborative exercise between graduate and undergraduate nursing students using a computer-assisted simulator in a mock cardiac arrest. Nursing Education Perspectives, 30(1), 22-27.

Brunt, B. A. (2005).Models, measurement, and strategies in developing critical-thinking skills. The Journal of Continuing Education in Nursing, 36(6), 255-262.

Burke, P. M. (2010). A simulation case study from an instructional design framework. Teaching and Learning in Nursing, 5, 73-77. Doi:10.1016/j.teln.2010.01.003

Burns, H., O'Donnell, J., \& Artman, J. (2010). High-fidelity simulation in teaching problem solving to $1^{\text {st }}$-year nursing students: A novel use of the nursing process. Clinical Simulation in Nursing, 6(3), e87-e95. Doi:10.1016/j.ecns.2009.07.005

Cant, R. P., \& Cooper, S. J. (2010). Simulation-based learning in nurse education: systematic review. Journal of Advanced Nursing, 66(1), 3-15. doi: 10.1111/j.13652648.2009.05240.x

Cantrell, M. A. (2008). The importance of debriefing in clinical simulations. Clinical Simulation in Nursing, 4(2), e19-e23. Doi:10.1016/j.ecns.2008.06.006

Cantrell, M. A., Meakim, C., \& Cash, K. (2008). Development and evaluation of three pediatricbased clinical simulation. Clinical Simulation in Nursing Education, 4(1), e21-e28. Doi:10.1016/j.ecns.2009.05.052

Carver, K., \& Marshall, P. L. (2010). Associate degree nursing education. In W. M. Nehring \& F. R. Lashley (Eds.), High fidelity patient simulation in nursing education (pp. 211-231). Sudbury, MA: Jones and Bartlett.

Cato, D. L., \& Murray, M. (2010). Use of simulation training in the intensive care unit. Critical Care Nursing Quarterly, 33(1), 44-51.

Chappy, S., Jambunathan, J., \& Marnocha, S. (2010). Evidence-based curricular strategies to enhance BSN graduates "e transition into practice. Nurse Educator, 35(1), 20-24.

Childs, J. C. \& Sepples, S. (2006). Clinical teaching by simulation: Lessons learned from a complex patient care scenario. Nursing Education Perspectives, 27(3), 154-158.

Cioffi, J. (2001). Clinical simulations: development and validation. Nurse Education Today, 21, 477-486. Doi:10.1054/nedt.2001.0584

Clarke, S. P., \& Aiken, L. H. (2003). Failure to rescue: Needless deaths are prime examples of the need for more nurses at the bedside. American Journal of Nursing, 103(1), 42-47. 
Cooper, J., \& Taqueti, V. (2004). A brief history of the development of mannequin simulators for clinical education and training. Quality and Safety in Health Care, 13, i11-i18. Retrieved from http://qualitysafety.bmj.com/content/13/suppl_1/i11.full.pdf+htmldoi:10.1136/qshc.2004. 009886

Comer, S. (2005). Patient care simulations: Role playing to enhance clinical understanding. Nursing Education Perspectives, 26(6), 357-361.

Cronenwett, L. R. (2011). Nursing education priorities for improving health and health care. In The Future of Nursing: Leading Change, Advancing Health (pp. 505-515). Washington, DC: The National Academies Press. Retrieved from http://www.nap.edu/openbook.php?record_id=12956\&page $=477$

Curtin, M. M., \& Dupuis, M. D. (2008). Development of human patient simulation programs: Achieving big results with a small budget. Journal of Nursing Education, 47(11), 522523.

Daly, W. (1998). Critical thinking as an outcome of nursing education. What is it? Why is it important to nursing practice? Journal of Advanced Nursing, 28(2), 323-331.

Daly, W. (2001). The development of an alternative method in the assessment of critical thinking as an outcome of nursing education. Journal of Advanced Nursing, 36(1), 120-130.

Davies, E. (1995). Reflective practice: a focus for caring. Journal of Nursing Education, 34(4), 167-174.

Decker, S. (2007a). Integrating guided reflection into simulated learning experiences. In P. R. Jeffries (Ed.), Simulation in nursing education: from conceptualization to evaluation (pp.73-85). New York, NY: NLN.

Decker, S. (2007b). Simulation as an educational strategy in the development of critical and reflective thinking: A qualitative exploration. (Unpublished doctoral dissertation). Texas Woman's University at Denton, TX.

Decker, S. (2007c). Simulation, Education, \& Ethics. In P. R. Jeffries (Ed.), Simulation in nursing education: from conceptualization to evaluation (pp. 11-19). New York, NY: National League for Nursing.

Decker, S., Sportsman, S., Puetz, L., \& Billings, L. (2008). The evolution of simulation and its contribution to competency. Journal of Continuing Education in Nursing, 39(2), 74-80.

Decker, S., Utterback, V., Thomas, M., Mitchell, M., \& Sportsman, S. ((2011). Assessing continued competency through simulation: A call for stringent action. Nursing Education Perspectives, 32(2), 120-125. 
De Giovanni, D., Roberts, T., \& Norman, G. (2009). Relative effectiveness of high- versus lowfidelity simulation in learning heart sounds. Medical Education, 43, 661-668. Doi:10.1111/j.1365-2923.2009.03398.x

Del Bueno, D. (2005). A crisis in critical thinking. Nursing Education Perspectives, 26(5), 278283.

Dewey, J. (1916). Democracy and education [HTML markup copyright 1994 ILT Digital Classics version]. Retrieved from http://www.ilt.columbia.edu/publications/dewey.html

Dewey, J. (1933). How we think: A restatement of the relation of reflective thinking to the educative process. Boston, MA: Heath.

Diener, E., \& Hobbs, N. (2012).Simulating care: Technology-mediated learning in twenty-first century nursing education. Nursing Forum, 47(1), 34-38.

Dillard, N., Sideras, S., Ryan, M., Carlton, K., Lasater, K., \& Siktberg, L. (2009). A collaborative project to apply and evaluate the Clinical Judgment Model through simulation. Nursing Education Perspectives, 30(2), 99-104.

Duchscher, J. (1999). Catching the wave: Understanding the concept of critical thinking. Journal of Advanced Nursing, 29(3), 577-583. doi: 10.1046/j.1365-2648.1999.00925.x

Duchscher, J. (2003). Critical Thinking: Perceptions of newly graduated female baccalaureate nurses. Journal of Nursing Education, 42(1), 14-27.

Durham, C. F., \& Alden, K. R. (2008). Enhancing patient safety in nursing education through patient simulation. In R. G. Hughes (Ed.), Patient safety and quality: an evidence-based handbook for nurses (Ch. 51). Rockville, MD: Agency for Healthcare Research and Quality. Retrieved from http://www.ncbi.nlm.nih.gov/books/NBK2628/

Elfrink, V. L., Kirkpatrick, B., Nininger, J., \& Schubert, C. (2010). Using learning outcomes to inform teaching practices in human patient simulation. Nursing Education Perspectives, 31(2), 97-100.

Elsevier HESI Assessment (2010). RN Exit Exam® Summary Report for West Virginia University-Parkersburg-ADN. Retrieved from https://hesiinet.com/

Elsevier, Review \& Testing. (2010). 2010 Scientific Evidence for Elsevier Testing \& Remediation. Houston, Texas: Elsevier.

Elsevier, Review \& Testing. (2011). 2011 Scientific Evidence for Elsevier HESI ${ }^{T M}$ Exams. $^{2}$ Houston, Texas: Elsevier.

Ennis, R. H. (1962). A concept of critical thinking. Harvard Educational Review, 32(1), 81-111. 
Facione, P. A. (1984). Toward a theory of critical thinking. Liberal Education, 70(3), 253-261.

Facione, P. A. (1990). Critical thinking: A statement of expert consensus for purposes of educational assessment and instruction [Executive Summary]. The Delphi report. Millbrae, CA: California Academic Press. Complete American Philosophical Association Delphi Research Report available as ERIC Doc. No: ED315423.

Facione, N. C., \& Facione, P. A. (1996). Externalizing the critical thinking in knowledge development and clinical judgment. Nursing Outlook, 44(3), 129-136.

Facione, N. C., \& Facione, P. A. (1997). Critical thinking assessment in nursing education programs: An aggregate data analysis. Millbrae, CA: California Academic Press.

Facione, N. C., \& Facione, P. A. (2008).Critical thinking and clinical judgment. In N. C. Facione \& P. A. Facione (Eds.), Critical thinking and clinical reasoning in the health sciences: A teaching anthology (pp. 1-13). Millbrae, CA: Insight Assessment/The California Academic Press.

Feingold, C. E., Calaluce, M., \& Kallen, M. A. (2004). Computerized patient model and simulated clinical experiences: Evaluation with baccalaureate nursing students. Journal of Nursing Education,43(4), 156-163.

Fero, L. J., O`Donnell, J. M., Zullo, T. G., Dabbs, A. D., Kitutu, J., Samosky, J. T., \& Hoffman, L. A. (2010). Critical thinking skills in nursing students: comparison of simulation-based performance with metrics. Journal of Advanced Nursing, 66(10), 2182-2193. Doi:10.1111/j.1365-2648.2010.05385.x

Field, A. (2009). Discovering statistics using SPSS ( $3^{\text {rd }}$ ed.). London:Sage Publications, Ltd.

Forehand, M. (2005). Bloom's taxonomy: Original and revised. In M. Orey (Ed.), Emerging perspectives on learning, teaching, and technology. Retrieved from http://projects.coe.uga.edu/epltt

Fountain, R., \& Alfred, D. (2009). Student satisfaction with high-fidelity simulation: Does it correlate with learning styles? Nursing Education Perspectives, 30(2), 96-98.

Gaba, D. M. (1992). Improving anesthesiologists "e performance by simulating reality. Journal of Anesthesiology, 76, 491-494.

Gaba, D. M. (2004). The future vision of simulation in health care. Quality and Safety in Health Care, 13, i2-i10. Retrieved from http://qualitysafety.bmj.com/content/13/suppl_1/i2.full doi:10.1136/qshc.2004.009878

Gant, L. T. (2007). Human Simulation in Emergency Nursing Education: Current Status. Journal of Emergency Nursing, 33(1), 69-71. 
Gantt, L.T., \& Webb-Corbett, R. (2010). Using simulation to teach patient safety behaviors in undergraduate nursing education. Journal of Nursing Education, 49(1), 48-51. Doi:10.3928/01484834-20090918-10

Garrett, B. (2005). Student nurses ${ }^{\text {ee }}$ perceptions of clinical decision-making in the final year of adult nursing studies. Nurse Education in Practice, 5, 30-39.doi: 10.1016/j.nepr.2004.03.003

Garrett, B., MacPhee, M., \& Jackson, C. (2010). High-Fidelity patient simulation: Considerations for effective learning. Nursing Education Perspectives, 31(5), 309-313.

Goodman, W. M., \& Lamers, A. (2010). Said another way: Asking the right questions regarding the effectiveness of simulations. Nursing Forum, 45(4), 246-252.

Gordon, C. J., and Buckley, T. (2009). The effect of high-fidelity simulation training on medicalsurgical graduate nurses ${ }^{\text {ee }}$ perceived ability to respond to patient clinical emergencies.

The Journal of Continuing Education in Nursing, 40(11), 491-498.

Doi:10.3928/00220124-20091023-06

Gordon, J. A., Wilkerson, W. M., Shaffer, D. W., \& Armstrong, E. G. (2001). "Practicing" medicine without risk: Students ${ }^{\text {ee }}$ and educators ${ }^{\text {ee }}$ responses to high-fidelity patient simulation. Academic Medicine, 76(5), 469-472.

Guhde, Jacqueline. (2010). Using online exercises and patient simulation to improve students ${ }^{\text {ee }}$ clinical decision-making. Nursing Education Perspectives, 31(6), 387-389.

Haffer, A. G., \& Raingruber, B. J. (1998). Discovering confidence in clinical reasoning and critical thinking development in baccalaureate nursing students. Journal of Nursing Education, 37(2), 61-70.

Hagbaghery, M. A., Salsali, M., \& Ahmadi, F. (2004). The factors facilitating and inhibiting effective clinical decision-making in nursing: A qualitative study. BioMed Central (BMC) Nursing, 3(2). doi:10.1186/1472-6955-3-2. Retrieved from http://www.biomedcentral.com/1472-6955/3/2

Halpern, D. F. (1989). Thought and knowledge: An introduction to critical thinking (2 ${ }^{\text {nd }}$ ed.). Hillsdale, N J: Lawrence Erlbaum Associates.

Hansten, R., \& Washburn, M. (2001). Outcomes-based care delivery. American Journal of Nursing, 101(2), 24A-24D.

Harder, B. N. (2009). Evolution of simulation use in health care education. Clinical Simulation in Nursing, 5(5), e169-e172. Doi:10.1016/j.ecns.2009.04.092 
Harder, B. N. (2010). Use of simulation in teaching and learning in health sciences: A systematic review. Journal of Nursing Education, 49(1), 23-28. Doi:10.3928/01484834-2009082808

Haskvitz, L. M., \& Koop, E. C. (2004). Students struggling in clinical? A new role for the patient simulator. Journal of Nursing Education, 43(4), 181-184.

Hauber, R. P., Cormier, E., \& Whyte, J. (2010). An exploration of the relationship between knowledge and performance-related variables in high-fidelity simulation: Designing instruction that promotes expertise in practice. Nursing Education Perspectives, 31(4), 242-246.

Hawkins, K., Todd, M., \& Manz, J. (2008). A unique simulation teaching method. Journal of Nursing Education, 47(11), 524-527.

Hayden, J. (2010). Use of simulation in nursing education: National survey results. Journal of Nursing Regulation, 1(3), 52-57.

Heaslip, P. (1993, Revised 2008). Critical thinking and nursing. Retrieved from http://www.criticalthinking.org/pages/index-of-articles/critical-thinking-and-nursing/834

Henneman, E.A., \& Cunningham, H. (2005). Using clinical simulation to teach patient safety in an acute/critical care nursing course. Nurse Educator, 30(4), 172-177.

Henneman, E. A., Cunningham, H., Roche, J. P., \& Cumin, M. E. (2007). Human patient simulation: Teaching students to provide safe care. Nurse Educator, 32(5), 212-217.

Herrmann, E. K. (1981). Mrs. Chase: A noble and enduring figure. American Journal of Nursing, $81,1836$.

Hicks, F. D. (2001). Critical thinking: Toward a nursing science perspective. Nursing Science Quarterly, 14(1), 14-21.doi: 10.1177/089431840101400106

Hicks, F., Coke, L., \& Li, S. (2009). Report of findings from the effect of high fidelity simulation on nursing students ${ }^{\text {ee }}$ knowledge and performance: A pilot study. NCSBN Research Brief, 40. Retrieved from https://www.ncsbn.org/09_SimulationStudy_Vol40_web_with_cover.pdf

Hoffmann, R., O'Donnell, J., \& Kim, Y. (2007). The effects of human patient simulators on basic knowledge in critical care nursing with undergraduate senior baccalaureate nursing students. Simulation in Healthcare, 2(2), 110-114.

Holaday, S. D., \& Buckley, K. M. (2008). Addressing challenges in nursing education through a clinical instruction model based on a hybrid, inquiry-based learning framework. Nursing Education Perspectives, 29(6), 353-358. 
Horan, K. (2009). Using the HUMAN PATIENT SIMULATOR to foster critical thinking in critical situations. Nursing Education Perspectives, 30(1), 28-30.

Hovancsek, M. T. (2007). Using simulation in nursing education. In P. R. Jeffries (Ed.), Simulation in nursing education: from conceptualization to evaluation (pp. 1-9). New York, NY: National League for Nursing.

Howard, V. (2007).A comparison of educational strategies for the acquisition of medicalsurgical nursing knowledge and critical thinking skills: Human patient simulator vs. the interactive case study approach (Unpublished doctoral dissertation). University of Pittsburgh, PA. Retrieved from http://challenger.library.pitt.edu/ETD/available/etd$\underline{04182007-205440 /}$

Howard, V. M., Englert, N., Kameg, K., \& Perozzi, K. (2011). Integration of simulation across the undergraduate curriculum: Student and faculty perspectives. Clinical Simulation in Nursing, 7(1), e1-e10. Doi:10.1016/j.ecns.2009.10.004

Hunter, C., \& Ravert, P. (2010). Nursing students ${ }^{\text {ee }}$ perceptions of learning outcomes throughout simulation experiences. Undergraduate Research Journal for the Human Sciences, 9. Retrieved from http://www.kon.org/urc/urc research journal9.html

Hyland, J. R., \& Hawkins, M. C. (2009). High-fidelity human simulation in nursing education: A review of literature and guide for implementation. Teaching and Learning in Nursing, 4, 14-21. Doi:10.1016/j.teln.2008.07.004

Ignatavicius, D. D. (2001). Six critical thinking skills for at-the-bedside success. Dimensions of Critical Care Nursing, 20(2), 30-33.

Institute of Medicine. (1999). To err is human: Building a safer health system. Washington, DC: National Academies Press. Retrieved from http://www.nap.edu/openbook.php?record id=9728\&page=1

Institute of Medicine. (2001). Crossing the quality chasm: A new health system for the $21^{\text {st }}$ century. Washington, DC: National Academies Press.

Institute of Medicine. (2003). Health professions education: A bridge to quality. Washington, DC: National Academies Press.

Institute of Medicine. (2004). Keeping patients safe: Transforming the work environment for nurses. Washington, DC: National Academies Press. Retrieved from http://www.nap.edu/openbook.php?record id=10851\&page=R1

Institute of Medicine. (2011). The future of nursing: leading change, advancing health. Committee on the Robert Wood Johnson Foundation Initiative on the future of nursing at the Institute of Medicine. Washington, DC: National Academies Press. Retrieved from http://www.nap.edu/openbook.php?record_id=12956\&page=R1 
Ironside, P. M. (1999). Thinking in nursing education. Part I: A student ex $^{\mathrm{s}}$ experience in learning to think. Nursing and Health Care Perspectives, 20(5), 238-242.

Ironside, P. M. (2005). Teaching thinking and reaching the limits of memorization: Enacting new pedagogies. Journal of Nursing Education, 44(10), 441-449.

Ironside, P. M., Jeffries, P. R., \& Martin, A. (2009). Fostering patient safety competencies using multiple-patient simulation experiences. Nursing Outlook, 57(6), 332-337.

Doi:10.1016/j.outlook.2009.07.010

Ironside, P. M., \& McNelis, A. M. (2010). Clinical education in pre-licensure nursing programs; results from an NLN national survey, 2009. New York, NY: National League for Nursing.

Irwin, R. E. (2011). The diffusion of human patient simulation into an associate degree in nursing curriculum. Teaching and Learning in Nursing, 6(4), 153-158.

Doi:10.1016/j.teln.2011.02.004

Issenberg, S. B., McGaghie, W. C., Hart, I. R., Mayer, J. W., Felner, J. M., \& Petrusa, E. R. (1999). Simulation technology for health care professional skills training and assessment. Journal of the American Medical Association, 282(9), 861-866. doi:101001/pubs.JAMA-ISSN-0098-7484-282-9-jsc90153

Issenberg, S. B., McGaghie, W. C., Petrusa, E. R., Gordon, D. L., \& Scalese, R. J. (2005). Features and uses of high-fidelity medical simulations that lead to effective learning: a BEME systematic review. Medical Teacher, 27(1), 10-28.

Doi:10.1080/01421590500046924

Issenberg, S. B., \& Scalese, R. J. (2007). Best evidence on high-fidelity simulation: what clinical teachers need to know. The Clinical Teacher, 4, 73-77.

Jacobson, L., \& Grindel, C. (2006). What is happening in pre-licensure RN clinical nursing education? Findings from the faculty and administrator survey on clinical nursing education [Headlines from the NLN]. Nursing education Perspectives, 27(2), 108-109.

Jarzemsky, P., McCarthy, J., \& Ellis, N. (2010). Incorporating quality and safety education for nurses competencies in simulation scenario design. Nurse Educator, 35(2), 90-92.

Jeffries, P. R. (2005). A framework for designing, implementing, and evaluating simulations used as teaching strategies in nursing. Nursing Education Perspectives, 26(2), 96-103.

Jeffries, P. R. (Ed.). (2007). Simulation in nursing education: From conceptualization to evaluation. New York, NY: National League for Nursing.

Jeffries, P. R. (2008). Getting in S.T.E.P. with simulations: Simulations take educator preparation. Nursing Education Perspectives, 29(2), 70-73. 
Jeffries, P. R. (2009). Dreams for the future of clinical simulation [Editorial]. Nursing Education Perspectives, 30(2), 71.

Jeffries, P. R., Bambini, D., Hensel, D., Moorman, M., \& Washburn, J. (2009). Constructing maternal-child learning experiences using clinical simulations. Journal of Gynecologic and Neonatal Nursing, 38, 613-623. Doi:10.1111/j.1552-6909.2009.01060.x

Jeffries, P. R., Clochesy, J. M., \& Hovancsek, M. T. (2009). Designing, implementing, and evaluating simulations in nursing education. In D. M. Billings \& J. A. Halstead (Eds.), Teaching in nursing: A guide for faculty ( ${ }^{\text {rd }}$ ed., pp. 322-334). St. Louis, MO: Saunders.

Jeffries, P. R., \& Rizzolo, M. A. (2006). Designing and implementing models for the innovative use of simulation to teach nursing care of ill adults and children: A national, multi-site, multi-method study [Summary Report]. New York, NY: National League for Nursing. Retrieved at http://www.nln.org/research/LaerdalReport.pdf

Jeffries, P. R., \& Rogers, K. J. (2007). Theoretical framework for simulation design. In P. R. Jeffries (Ed.), Simulation in nursing education: from conceptualization to evaluation (pp. 21-33). New York, NY: National League for Nursing.

Jeffries, P. R., Woolf, S., \& Linde, B. (2003).Technology-based vs. traditional instruction. A comparison of two methods for teaching the skill of performing a 12 lead ECG. Nursing Education Perspectives, 24(2), 70-74.

Johnson, J. H., Zerwic, J. J., \& Theis, S. L. (1999). Clinical simulation laboratory: An adjunct to clinical teaching. Nurse Educator, 24(5), 37-41.

Johnson-Russell, J., \& Bailey, C. (2010).Facilitated Debriefing.I n W. M. Nehring \& F. R. Lashley (Eds.), High fidelity patient simulation in nursing education (pp. 369-385). Sudbury, MA: Jones and Bartlett Publications

Jones, S. A., \& Brown, L. N. (1991). Critical thinking: impact on nursing education. Journal of Advanced Nursing, 16, 529-533.

Kameg, K., Clochesy, J., Mitchell, A. M., \& Suresky, J. M. (2010). The impact of high fidelity human simulation on self-efficacy of communication skills. Issues in Mental Health Nursing, 31, 315-323. Doi:10.3109/01612840903420331

Kameg, K., Mitchell, A. M., Clochesy, J., Howard, V. M., \& Suresky, J. (2009). Communication and human patient simulation in psychiatric nursing. Issues in Mental Health Nursing, 30, 503-508. Doi:10.1080/01612840802601366

Kardong-Edgren, S., Adamson, K. A., \& Fitzgerald, C. (2010). A review of currently published evaluation instruments for human patient simulation. Clinical Simulation in Nursing, 6(1), e25-e35. Doi:10.1016/j.ecns.2009.08.004 
Kardong-Edgren, S., Anderson, M., \& Michaels, J. (2007). Does simulation fidelity improve student test scores? Clinical Simulation in Nursing, 3(1), e21-e24.

Doi:10.1016/j.ecns.2009.05.035

Kardong-Edgren, S., Lungstrom, N., \& Bendel, R. (2009). VitalSim ${ }^{\circledR}$ vs. SimMan®: A comparison of BSN student test scores, knowledge retention, and satisfaction. Clinical Simulation in Nursing, 5(3), e105-e111. Doi:10.1016/j.ecns.2009.01.007

Kardong-Edgren, S. E., Starkweather, A. R., \& Ward, L. D. (2008). The integration of simulation into a clinical foundations of nursing course: Student and faculty perspectives. International Journal of Nursing Education Scholarship, 5(1), Article 26.

Kataoka-Yahiro, M., \& Saylor, C. (1994).A critical thinking model for nursing judgment. Journal of Nursing Education, 33(8), 351-356.

Katz, G. B., Peifer, K. L., \& Armstrong, G. (2010). Assessment of patient simulation use in selected baccalaureate nursing programs in the United States. Simulation in Healthcare, 5(1), 46-51. Doi:10.1097/SIH.0b013e3181ba1f46

Kenner, C. A., \& Pressler, J. L. (2011).Trends in nursing education. Nurse Educator, 36(5), 179180.

Kenwood, K., \& Zhong, E. H. (2006). Report of findings from the practice and professional issues survey fall 2004. NCSBN Research Brief, 22. Retrieved from https://www.ncsbn.org/Vol_22_web.pdf

Kneebone, R. L., Scott, W., Darzi, A., \& Horrocks, M. (2004). Simulation and clinical practice: strengthening the relationship. Medical Education, 38, 1095-1102. Doi:10.1111/j.13652929.2004.01959.x

Kolb, D. A. (1984). Experiential learning: Experience as the source of learning and development. Englewood Cliffs, NJ: Prentice-Hall.

Kovner, C. T., Brewer, C. S., Yingrengreung, S., \& Fairchild, S. (2010). New nurses ${ }^{\text {ee }}$ views of quality improvement education. The Joint Commission Journal on Quality\& Patient Safety, 36(1), 29-35.

Kuiper, R., Heinrich, C., Matthias, A., Graham, M. J., \& Bell-Kotwall, L. (2008). Debriefing with the OPT model of clinical reasoning during high fidelity patient simulation. International Journal of Nursing Education Scholarship, 5(1), Article 17.

Kuiper, R. A., \& Pesut, D. J. (2004). Promoting cognitive and metacognitive reflective reasoning skills in nursing practice: self-regulated learning theory. Journal of Advanced Nursing, 45(4), 381-391. 
Kuznar, K. (2007). Associate degree nursing students ${ }^{\text {ee }}$ perceptions of learning using a highfidelity human patient simulator. Teaching and Learning in Nursing, 2(2), 46-52. Doi:10.1016/j.teln.2007.01.009

Laerdal.(2007). Simulation in Nursing Education [Computer software]. New York, NY: NLN/Laerdal Medical Corporation

Laerdal. (2012a). History: Laerdal yesterday and today. Retrieved from www.laerdal.com/us/doc/367/History

Laerdal. (2012b). Products and Services: Medical Education. Retrieved from http://www.laerdal.com/ca/nav/36/Medical-Education

Laerdal. (2012c). Products and Services: VitalSim. Retrieved from www.laerdal.com/us/doc/247/VitalSim\#

Landis, R. E., \& Michael, W. B. (1981). The factorial validity of three measures of critical thinking within the context of Guilford 's Structure-of-Intellect Model for a sample of ninth grade students. Educational and Psychological Measurement, 41(4), 1147-1166. doi: $10.1177 / 001316448104100424$

Lapkin, S., \& Levett-Jones, T. (2011). A cost-utility analysis of medium vs. high-fidelity human patient simulation mannequins in nursing education. Journal of Clinical Nursing, 20, 3543-3552. Doi:10.1111/j.1365-2702.2011.03843.x

Lapkin, S., Levett-Jones, T., Bellchambers, H., \& Fernandez, R. (2010). Effectiveness of patient simulation mannequins in teaching clinical reasoning skills to undergraduate nursing students: A systematic review. Clinical Simulation in Nursing, 6(6), e207-e222.

Doi:10.1016/j.ecns.2010.05.005

Larew, C., Lessans, S., Spunt, D., Foster, D., \& Covington, B. (2006). Innovations in clinical simulation: Application of Benner's theory in an interactive patient care simulation. Nursing Education Perspectives, 27(1), 16-21.

Lasater, K. (2007). High-fidelity simulation and development of clinical judgment: Students ${ }^{\text {ee }}$ experiences. Journal of Nursing Education, 46(6), 269-276.

Laschinger, S., Medves, J., Pulling, C., McGraw, R., Waytuck, B., Harrison, M. B., \& Gambeta, K. (2008). Effectiveness of simulation on health profession students "e knowledge, skills, confidence and satisfaction. International Journal of Evidence Based Healthcare, 6, 278302. Doi:10.1111/j.1479-6988.2008.00108.x

Leigh, G. T. (2008). High-fidelity patient simulation and nursing studentse ${ }^{\text {ee }}$ self-efficacy: A review of the literature. International Journal of Nursing Education Scholarship, 5(1), Article 37. 
Lewis, D. Y., \& Ciak, A. D. (2011). The impact of a simulation lab experience for nursing students. Nursing Education Perspectives, 32(4), 256-258.

Li, S. (2008, March). The role of simulation in nursing education: A regulatory perspective. Presentation at the National Council of State Boards of Nursing Faculty Shortage Conference. Chicago, IL. PowerPoint ${ }^{\text {ee }}$ retrieved from https://www.ncsbn.org/Suling2.ppt

Li, S., \& Kenwood, K. (2006). A national survey on elements of nursing education. NCSBN Research Brief, 24. Retrieved from https://www.ncsbn.org/Vol_24_web.pdf

Limoges, J. (2010). An exploration of ruling relations and how they organize and regulate nursing education in the high-fidelity patient simulation laboratory. Nursing Inquiry, 17(1), 57-63.

Lisko, S. A., \& O'Dell, V. (2010). Integration of theory and practice: Experiential learning theory and nursing education. Nursing Education Perspectives, 31(2), 106-108.

Long, K. A. (2004). Preparing nurses for the $21^{\text {st }}$ century; Reenvisioning nursing education and practice. Journal of Professional Nursing, 20(2), 82-88. Doi:10.1016/j.profnurs.2004.02.002

Lundberg, K. M. (2008). Promoting self-confidence in clinical nursing students. Nurse Educator, $33(2), 86-89$.

Martin, C. (2002). The theory of critical thinking of nursing. Nursing Education Perspectives, 23(5), 243-247.

McCallum, J. (2007). The debate in favour of using simulation education in pre-registration adult nursing. Nurse Education Today, 27(8), 825-831. Doi:10.1016/j.nedt.2006.10.014

McCausland, L. L., Curran, C. C., \& Cataldi, P. (2004). Use of a human simulator for undergraduate nurse education. International Journal of Nursing Education Scholarship, 1(1), Article 23.

McGaghi, W. C., Issenberg, S. B., Petrusa, E. R., \& Scalese, R. J. (2006). Effect of practice on standardized learning outcomes in simulation-based medical education. Medical Education, 40(1), 792-797.

McGaghi, W. C., Issenberg, S. B., Petrusa, E. R., \& Scalese, R. J. (2010). A critical review of simulation-based medical education research: 2003-2009. Medical Education, 44, 50-63.

McLellen, H. (1996). Situated learning perspectives. Englewood Cliffs, NJ: Educational Technology Publications. 
McMillan, J. H. (1987). Enhancing college students ${ }^{\text {ee }}$ critical thinking: A review of studies. Research in Higher Education, 26(1), 3-29.

McPeck, J. E. (1981). Critical thinking and education, New York, NY: St. Martin's Press.

Medical Education Technologies, Inc. (2012). Human Patient Simulator: HPS. Retrieved from http://www.meti.com/products

Medley, C., \& Horne, C. (2005). Using simulation technology for undergraduate nursing education. Journal of Nursing Education, 44(1), 31-34.

Morrison, S., Adamson, C., Nibert, A., \& Hsia, S. (2004). HESI exams: An overview of reliability and validity. CIN: Computers, Informatics, Nursing, 22(4), 220-226.

Morrison, S., \& Free, K. (2001). Writing multiple-choice test items that promote and measure critical thinking. Journal of Nursing Education,40(1), 17-24.

Morrison, S., Nibert, A., \& Flick, J. (2006). Critical Thinking and Test Item Writing (2 ${ }^{\text {nd }}$ ed.). Houston, TX: Health Education Systems, Inc.

Morton, P. (1995). Creating a laboratory that simulates the critical care environment. Critical Care Nurse, 16(6), 76-81.

Mould, J., White, H., \& Gallagher, R. (2011).Evaluation of a critical care simulation series for undergraduate nursing students. Contemporary Nurse, 38(1-2), 180-190.

Murphy, J. I. (2004). Using focused reflection and articulation to promote clinical reasoning: An evidence-based teaching strategy. Nursing Education Perspectives, 25(5), 226-231.

Napier, D. (2007). 2007 annual report and recommendations. Charleston, WV: West Virginia Center for Nursing.

National Council of State Boards of Nursing.(2005). Clinical instruction in pre-licensure nursing programs. Retrieved from https://www.ncsbn.org/Final Clinical Instr Pre Nsg programs.pdf

National Council of State Boards of Nursing.(2006). Evidence-based nursing education for regulation ( $E B N E R)$. Retrieved from https://www.ncsbn.org/Final_06_EBNER_Report.pdf

National Council of State Boards of Nursing. (2009a). Innovations in education regulation report: background and literature review. Retrieved from https://www.ncsbn.org/Innovations_Report.pdf

National Council of State Boards of Nursing. (n.d.). Simulation survey update. Received from WV Board of Registered Professional Nurses, November, 2010. 
National Council of State Boards of Nursing. (2010). NCSBN simulation study: Assessing outcomes of simulated clinical experiences in nursing education. NCSBN Simulation Study Executive Summary. Received from NCSBN August, 2010.

National Council of State Boards of Nursing. (2011). Licensure of nurses: An integral part of public protection. Retrieved from https://www.ncsbn.org/LicensureofNurses_Aug2011.pdf

National League for Nursing. (2000). Educational competencies for graduates of associate degree nursing programs. New York, NY: Author.

National League for Nursing. (2003). Innovation in nursing education: A call to reform [Position Statement]. New York, NY: Author. Retrieved from http://www.nln.org/aboutnln/PositionSTatements/innovation082203.pdf

National League for Nursing.(2005a). Core competencies of nurse educators with task statements. New York, NY: Author. Retrieved from http://www.nln.org/facultyprograms/pdf/corecompetencies.pdf

National League for Nursing. (2005b). Transforming nursing education [Position Statement]. New York, NY: Author. Retrieved from http://www.nln.org/aboutnln/PositionStatements/transforming052005.pdf

National League for Nursing. (2010). Outcomes and competencies for graduates of practical/vocational, diploma, associate degree, baccalaureate, master's, practice doctorate, and research doctorate programs in nursing. New York, NY: Author. Retrieved from http://www.nln.org/facultyprograms/competencies/comp ad dp.htm

National League for Nursing.(2011). Findings from the annual survey of schools of nursing academic year 2009-2010 [Executive Summary]. Retrieved from http://www.nln.org/research/slides/exec_summary_0910.pdf

National League for Nursing Accrediting Commission, Inc. (2008). NLNAC accreditation manual. Retrieved from http://www.nInac.org/manuals/Manual2008.htm

Nehring, W. M. (2008). U.S. boards of nursing and the use of high-fidelity patient simulators in nursing education. Journal of Professional Nursing, 24(2), 109-117.

Doi:10.1016/j.profnurs.2007.06.027

Nehring, W. M. (2010). A synthesis of theory and nursing research using high-fidelity patient simulation. In W. M. Nehring \& F. R. Lashley (Eds.), High-fidelity patient simulation in nursing education (pp. 27-56). Sudbury, MA: Jones and Bartlett Publications.

Nehring, W. M., Ellis, W. E., \& Lashley, F. R. (2001). Human patient simulators in nursing education: An overview. Simulation and Gaming, 32(2), 194-204. 
Nehring, W. M., \& Lashley, F. R. (2004a). Current use and opinions regarding human patient simulators in nursing education: An international survey. Nursing Education Perspectives, 25(5), 244-248.

Nehring, W. M., \& Lashley, F. R. (2004b).Using the human patient simulators in nursing education. Annual review of nursing education, 2, 163-181.

Nehring, W. M., \& Lashley, F.R. (2009). Nursing simulation: A review of the past 40 years. Simulation \& Gaming, 40(4), 528-552. Doi:10.1177/1046878109332282

Nehring, W. M., \& Lashley, F. R. (2010). High-fidelity patient simulation in nursing education. Sudbury, MA: Jones and Bartlett Publications.

Nehring, W. M., Lashley, F. R., \& Ellis, W. E. (2002).Critical incident nursing management using human patient simulators. Nursing Education Perspectives, 23, 128-132.

Niemer, L., Pfendt, K., \& Gers, M. (2010). Problem-based learning in nursing education: A process for scenario development. Nurse Educator, 35(2), 69-73.

Norman, G. (2003). Simulation-Saviour or Satan [Editorial]? Advances in Health Sciences Education, 8, 1-3.

Oermann, M. H. (1997). Evaluating critical thinking in clinical practice. Nurse Educator, 22(5), $25-28$.

Olejniczak, E., Schmidt, N., \& Brown, J. (2010). Simulation as an orientation strategy for new nurse graduates: An integrative review of the evidence. Simulation in Healthcare, 5(1), 52-57. Doi:10.1097/SIH.0b013e3181ba1f61

O'Neill, E. S., \& Dluhy, N. M. (1997). A longitudinal framework for fostering critical thinking and diagnostic reasoning. Journal of Advanced Nursing, 26, 825-832.

Ozturk, C., Muslu, G. K., \& Dicle, A. (2008).A comparison of problem-based and traditional education on nursing students ${ }^{\text {ee }}$ critical thinking dispositions. Nurse Education Today, 28, 627-632. Doi:10.1016/j.nedt.2007.10.001

Pacsi, A. (2008).Human simulators in nursing education. Journal of the New York State Nurses Association, Fall/Winter, 8-11.

Patterson, C., Crooks, D., \& Lunyk-Child, O. (2002). A new perspective on competencies for self-directed learning. Journal of Nursing Education, 41(1), 25-31.

Paul, R. (1995). Critical thinking: How to prepare students for a rapidly changing world (4thed.). Santa Rosa, CA: Foundation for Critical Thinking. 
Paul, R. W., \& Heaslip, P. (1995).Critical thinking and intuitive nursing practice. Journal of Advanced Nursing, 22, 40-47.

Pellico, L. H., Brewer, C. S., \& Kovner, C. T. (2009). What newly licensed registered nurses have to say about their first experiences. Nursing Outlook, 57(4), 194-203.

Doi:10.1016/j.outlook.2008.09.008

Pesut, D. J., \& Herman, J. (1999). Clinical reasoning: The art and science of critical and creative thinking. Albany, NY: Delmar.

Peteani, L. A. (2004). Enhancing clinical practice and education with high-fidelity human patient simulators. Nurse Educator, 29(1), 25-30.

Prion, S. (2008). A practical framework for evaluating the impact of clinical simulation experiences in pre-licensure nursing education. Clinical Simulation in Nursing, 4(3), e69e78. Doi:10.1016/j.ecns.2008.08.002

Radhakrishnan, K., Roche, J., \& Cunningham, H. (2007). Measuring clinical practice parameters with human patient simulation: A pilot study. International Journal of Nursing Education Scholarship, 4(1), Article 8.

Rapps, J., Riegel, B., \& Glaser, D. (2001). Testing a predictive model of what makes a critical thinker. Western Journal of Nursing Research, 23(6), 610-626.

Rauen, C. A. (2001). Using simulation to teach critical thinking skills: You can et just throw the book at them. Critical Care Nursing Clinics of North America, 13(1), 93-103.

Rauen, C. A. (2004). Simulation as a teaching strategy for nursing education and orientation in cardiac surgery. Critical Care Nurse, 24(3), 46-51.

Ravert, P. (2008). Patient simulator sessions and critical thinking. Journal of Nursing Education, 47(12), 557-562.

Reese, C. E., Jeffries, P. R., \& Engum, S. A. (2010). Learning together: Using simulations to develop nursing and medical student collaboration. Nursing Education Perspectives, 31(1), 33-37.

Reznek, M. A. (2004). Current status of simulation in education and research. In G. E. Loyd, C. L. Lake, \& R. B. Greenberg (Eds.), Practical health care simulations (pp. 27-47). Philadelphia, PA: Elsevier/Mosby

Rhodes, M. L., \& Curran, C. (2005). Use of the human patient simulator to teach clinical judgment skills in a baccalaureate nursing program. CIN:Computers, Informatics, Nursing, 23(5), 256-262. 
Rose, S., Courey, T., Ball, M., Bowler, C., \& Thompson, Z. (2012). Bringing simulation to life through a therapeutic encounter: a pedagogical approach used for associate degree nursing students. Teaching and Learning in Nursing, 7(1), 2-5.

Doi:10.1016/j.teln.2011.09.003

Rosen, K. R. (2004). The history of medical simulation. In G. E. Loyd, C. L. Lake, \& R. B. Greenberg (Eds.), Practical health care simulations (pp. 3-26). Philadelphia, PA: Elsevier.

Rowles, C. J., \& Russo, B. L. (2009). Strategies to promote critical thinking and active learning. In D. M. Billings \& J. A. Halstead (Eds.), Teaching in nursing: A guide for faculty $\left(3^{\mathrm{rd}}\right.$ ed., pp. 238-261). St. Louis, MO: Saunders/Elsevier.

Ruth-Sahd, L. A. (2003). Reflective practice: A critical analysis of data-based studies and implications for nursing education. Journal of Nursing Education, 42(11), 488-497.

Rystedt, H., \& Lindstrom, B. (2001). Introducing simulation technologies in nurse education: A nursing practice perspective. Nurse Education in Practice, 1(3), 134-141.

Salas, E., DiazGranados, D., Weaver, S., \& King, H. (2008). Does team training work? Principles for health care. Academic Emergency Medicine, 11, 1002-1009.

Schank, M. J. (1990). Wanted: nurses with critical thinking skills. The Journal of Continuing Education in Nursing, 21(2), 86-89.

Scheffer, B., \& Rubenfeld, M. (2000). A consensus statement on critical thinking in nursing. Journal of Nursing Education, 39(8), 352-359.

Scherer, Y. K., Bruce, S. A., Graves, B. T., \& Erdley, W. S. (2003). Acute care nurse practitioner education: Enhancing performance through the use of clinical simulation. AACN Clinical Issues: Advanced Practice in Acute \& Critical Care, 14(3), 331-341.

Scherer, Y. K., Bruce, S. A., \& Runkawatt, V. (2007).A comparison of clinical simulation and case study presentation on nurse practitioner students ${ }^{\text {ee }}$ knowledge and confidence in managing a cardiac event. International Journal of Nursing Education Scholarship, 4(1), 1-14, Article 22.

Schlairet, M. C., \& Pollock, J. W. (2010). Equivalence testing of traditional and simulated clinical experiences: Undergraduate nursing students ${ }^{\text {ee }}$ knowledge acquisition. Journal of Nursing Education, 49(1), 43-47. Doi:10.3928/01484834-20090918-08

Schoening, A. M., Sittner, B. J., \& Todd, M. J. (2006). Simulated clinical experience: Nursing students $^{\text {ee }}$ perceptions and the educators ${ }^{\text {ee }}$ role. Nurse Educator, 31(6), 253-258.

Schön, D. A. (1983).The reflective practitioner: How professionals think in action. New York, NY: Basic Books. 
Schön, D. A. (1987). Educating the reflective practitioner. San Francisco, CA: Jossey-Bass.

Schumacher, L. B. (2004). The impact of using high-fidelity computer simulation on critical thinking abilities and learning outcomes in undergraduate nursing students. Dissertation Abstracts International, 65(10B), 2567B (ISBN No. 0-496-11045-4). Retrieved from http://digital.library.duq.edu/cdm4/document.php?CISOROOT=/etd\&CISOPTR=77666

Sears, K., Goldsworthy, S., \& Goodman, W. M. (2010). The relationship between simulation in nursing education and medication safety. Journal of Nursing Education, 49(1), 52-55. Doi:10.3928/01484834-20090918-12

Sedlak, C. A. (1997). Critical thinking of beginning baccalaureate nursing students during the first clinical nursing course. Journal of nursing education, 36(1), 11-18

Seropian, M. A., Brown, K., Gavilanes, J. S., \& Driggers, B. (2004). Simulation: Not just a mannequin. Journal of Nursing Education, 43(4), 164-169.

Seymour, B., Kinn, S., \& Sutherland, N. (2003). Valuing both critical and creative thinking in clinical practice: narrowing the research-practice gap? Journal of Advanced Nursing, 42(3), 288-296.

Shepherd, I. A., Kelly, C. M., Skene, F. M., \& White, K. T. (2007). Enhancing graduate nursese health assessment knowledge and skills using low-fidelity adult human simulation. Simulation in Healthcare, 2(1), 16-24.

Shin, K. R., Lee, J. H., Ha, J. Y., \& Kim, K. H. (2006). Critical thinking dispositions in baccalaureate nursing students. Journal of Advanced Nursing, 56(2), 182-189. doi:10.1111/j.1365-2648.2006.03995.x

Shinnick, M. A., Woo, M. A., \& Mentes, J. C. (2011). Human patient simulation: State of the science in pre-licensure nursing education. Journal of Nursing Education, 50(2), 65-72. Doi:10.3928/01484834-20101230-01

Shirrell, D. (2008). Critical thinking as a predictor of success in an associate degree nursing program. Teaching and Learning in Nursing, 3(4), 131-136.

Siefert, J., Hinds, M., Yoho, M. J., Nibert, A. T., \& Morrison, S. (n.d.). Evolve Reach Admissions Assessment $A^{2}$ Exam. Houston, Texas: Elsevier.

Simmons, B., Lanuza, D., Fonteyn, M., Hicks, F., \& Holm, K. (2003). Clinical reasoning in experienced nurses. Western Journal of Nursing Research, 25(6), 701719.doi:10.1177/0193945903253092

Simpson, E., \& Courtney, M. (2002). Critical thinking in nursing education: Literature review. International Journal of Nursing Practice, 8, 89-90. 
Sinclair, B., \& Ferguson, K. (2009). Integrating simulated teaching/learning strategies in undergraduate nursing education. International Journal of Nursing Education Scholarship, 6(1), Article 7.

Sleeper, J. A., \& Thompson, C. (2008). The use of hi fidelity simulation to enhance nursing students ${ }^{\text {ee }}$ therapeutic communication skills. International Journal of Nursing Education Scholarship, 5(1), Article 42.

Smith, M. M. (2009). Creative clinical solutions: Aligning simulation with authentic clinical experiences. Nursing Education Perspectives, 30(2), 126-128.

Smith, J., \& Crawford, L. (2003). Report of findings from the practice and professional issues survey spring 2002. NCSBN Research Brief, 7. Retrieved from https://www.ncsbn.org/PPI_spr02_vol7.pdf

Smith, S. J., \& Roehrs, C. J. (2009). High-fidelity simulation: Factors correlated with nursing student satisfaction and self-confidence. Nursing Education Perspectives, 30(2), 74-78.

Sportsman, S., Schumacker, R. E., \& Hamilton, P. (2011). Evaluating the impact of scenariobased high-fidelity patient simulation on academic metrics of student success. Nursing Education Perspectives, 32(4), 259-265.

Spunt, D. (2007). Setting up a simulation laboratory. In P. R. Jeffries (Ed.), Simulation in nursing education: From conceptualization to evaluation (pp. 105-122). New York, NY: National League for Nursing.

Spunt, D., Foster, D., \& Adams, K. (2004). Mock code: A clinical simulation module. Nurse Educator, 29(5), 192-94.

Starkweather, A. R., \& Kardong-Edgren, S. (2008). Diffusion of innovation: Embedding simulation into nursing curricula. International Journal of Nursing Education Scholarship, 5(1), Article 13.

Steadman, R. H., Coates, W. C., Huang, Y. M., Matevosian, R., Larmon, B. R., McCullough, L., \& Ariel, D. (2006). Simulation-based training is superior to problem-based learning for acquisition of critical assessment and management skills. Critical Care Medicine, 34(1), 151-157. Doi:10.1097/01.CCM.0000190619.42013.94

Stefanski, R. R., \& Rossler, K. L. (2009). Preparing the novice critical care nurse: A communitywide collaboration using the benefits of simulation. The Journal of Continuing Education in Nursing, 40(10), 443-451. Doi:10.3928/00220124-20090923-03

Stein, D. (1998). Situated learning in adult education. Retrieved from ERIC digest http://www.ericdigests.org/1998-3/adult-education.html 
Su, W. M., \& Juestel, M. J. (2010). Direct teaching of thinking skills using clinical simulation. Nurse Educator, 35(5), 197-204. Doi:10.1097/NNE.0b013e3181e33969

Tagliareni, M. E. (2009). Beyond the realities of current practice: Preparing students to provide safe and effective care [President's message]. Nursing Education Perspectives, 30(2), 69.

Tanda, R., \& Denham, S. A. (2009). Clinical instruction and student outcomes. Teaching and Learning in Nursing, 4(4), 139-147. Doi:10.1016/j.teln.2009.01.002

Tanner, C. A. (2006a). The next transformation: Clinical education [Editorial]. Journal of Nursing Education, 45(4), 99-100.

Tanner, C. A. (2006b). Thinking like a nurse: A research-based model of clinical judgment in nursing. Journal of Nursing Education, 45(6), 204-211.

Tanner, C. A. (2008). Clinical judgment and evidence-based practice: Toward pedagogies of integration [Editorial]. Journal of Nursing Education, 47(8), 335-336.

Teekman, B. (2000). Exploring reflective thinking in nursing practice. Journal of Advanced Nursing, 31(5), 1125-1135.

Tiwari, A., Lai, P., So, M., \& Yuen, F. (2006). A comparison of the effects of problem-based learning and lecturing on the development of students ${ }^{\text {ee }}$ critical thinking. Medical Education, 40, 547-554. Doi:10.1111/j.1365-2929.2006.02481.x

Tuoriniemi, P., \& Schott-Baer, D. (2008).Implementing a high-fidelity simulation program in a community college setting. Nursing Education Perspectives, 29(2), 105-109.

Turner, P. (2005). Critical thinking in nursing education and practice as defined in the literature. Nursing Education Perspectives, 26(5), 272-277.

Twibell, R., Ryan, M., \& Hermiz, M. (2005).Faculty perceptions of critical thinking in student clinical experiences. Journal of Nursing Education, 44(2), 71-79.

United States Medical Licensing Examination.(2011). Bulletin of information. Retrieved from http://www.usmle.org/step-2-cs/

Vandrey, C., \& Whitman, K. (2001). Simulator training for novice critical care nurses: Preparing providers to work with critically ill patients. American Journal of Nursing, 101(9), 24GG-24LL.

Waldner, M. H., \& Olson, J. K. (2007). Taking the patient to the classroom: Applying theoretical frameworks to simulation in nursing education. International Journal of Nursing Education Scholarship, 4(1), Article 18. 
Walker, P. H., \& Redman, R. (1999). Theory-guided, evidence-based reflective practice. Nursing Science Quarterly, 12(4), 298-303.

Ward-Smith, P. (2008). The effect of simulation learning as a quality initiative. Urologic Nursing, 28(6), 471-473.

Watson, G., \& Glaser, E. M. (1991). Watson-Glaser Critical Thinking Appraisal Manual. Kent, $\mathrm{OH}$ : The Psychological Corporation.

Waxman, K. T. (2010). The development of evidence-based clinical simulation scenarios: Guidelines for nurse educators. Journal of Nursing Education, 49(1), 29-35. Doi:10.3928/01484834-20090916-07

Weaver, A. (2011). High-Fidelity patient simulation in nursing education: An integrative review. Nursing Education Perspectives, 32(1), 37-40.

Weis, P. A., \& Guyton-Simmons, J. (1998). A computer simulation for teaching critical thinking skills. Nurse Educator, 23(2), 30-33.

Weller, J. M. (2004). Simulation in undergraduate medical education: bridging the gap between theory and practice. Medical Education, 38, 32-38. Doi:10.1046/j.13652923.2004.01739.x

Wenk, M., Waurick, R., Schotes, D., Wenk, M., Gerdes, C., Van Aken, H., \& Popping, D. (2009). Simulation-based medical education is no better than problem-based discussions and induces misjudgment in self-assessment. Advances in Health Science Education, 14, 159-171. Doi:10.1007/s10459-008-9098-2

West Virginia University at Parkersburg (2012). WVU Parkersburg Associate Degree Nursing Program Student Nurse Handbook: Spring 2012. Parkersburg, WV: Author.

White, A. H. (2003). Clinical decision-making among fourth-year nursing students: An interpretive study. Journal of Nursing Education, 42(3), 113-120.

Wilson, M., Shepherd, I., Kelly, C., \& Pitzner, J. (2005). Assessment of a low-fidelity human patient simulator for the acquisition of nursing skills. Nurse Education Today, 25(1), 5667.

Wotton, K., Davis, J., Button, D., \& Kelton, M. (2010). Third-year undergraduate nursing studentse $^{\text {ee }}$ perceptions of high-fidelity simulation. Journal of Nursing Education, 49(11), 632-639. Doi:10.3928/01484834-20100831-01

Yoo, M. S., \& Yoo, I. Y. (2003). The effectiveness of standardized patients as a teaching method for nursing fundamentals. Journal of Nursing Education, 42(10), 444-448. 
Young, A. \& Langford, R. (2011). The Eighth $E^{2}$ Validity Study for RNs: Accuracy, Benchmarking, Remediation and Testing Practices Summary Report [Flyer]. Houston, Texas: Elsevier

Yuan, H. B., Williams, B. A., \& Fang, J. B. (2012). The contribution of high-fidelity simulation to nursing students' confidence and competence: a systematic review. International Nursing Review, 59(1), 26-33.

Ziv, A., Wolpe, P. R., Small, S. D., \& Glick, S. (2003). Simulation-based medical education: An ethical imperative. Academic Medicine, 78(8), 783-788. 
Appendix A

Program of Study 


\section{Associate in Applied Science Degree}

\section{Nursing}

(Prerequisite)

CHEM 111

First Year

FIRST SEMESTER

Course

NURS 111

ENGL 101

PSYC 101

BIOL 107

TOTAL
Credit

$4 \mathrm{hrs}$.

\section{SECOND SEMESTER}

Course

NURS 112

NURS 131

PSYC 241

BIOL 108

TOTAL
Medical Surgical Nursing 2

Physical Assessment

Introduction to Human Development

Anatomy and Physiology 2
Credit

9 hrs.

$3 \mathrm{hrs}$.

$3 \mathrm{hrs}$.

$4 \mathrm{hrs}$.

19 hrs.

Second Year

THIRD SEMESTER

Course

NURS 213

ENGL 102*

SOC $101^{*}$

TOTAL

Family-Centered Nursing

Composition 2

Introduction to Sociology
Credit

9 hrs.

$1 \mathrm{hr}$.

$3 \mathrm{hrs}$.

4 hrs.

\section{FOURTH SEMESTER}

\section{Course}

NURS 216

NURS 224

BIOL 200*

NURS 217

TOTAL

TOTAL CREDIT HOURS
Medical Surgical Nursing 3

Perspectives of Nursing

Microbiology

NCLEX-RN Review
Credit

$9 \mathrm{hrs}$.

3 hrs.

$\underline{3 \mathrm{hrs}}$.

15 hrs.

Credit

9 hrs.

$2 \mathrm{hrs}$.

4 hrs.

$\underline{1 \mathrm{hr}}$

$16 \mathrm{hrs}$

71 hrs.

* All required general education courses are pre or co-requisites of the final nursing course 
Appendix B

NURS 216 HPS Scenarios

$2006 / 2007$ 


\section{West Virginia University Parkersburg \\ Health Sciences Division \\ Department of Nursing \\ Nursing 216 HPS Scenarios 2006-2007 \\ Debbie Murphy, MSN, RN}

1. 40 yo female w/ L lobectomy for Ca. L chest tube to $20 \mathrm{~cm}$ suction, trach to T-piece with $40 \% \mathrm{O} 2$.

Assessment findings students should note: decreased breath sounds, trach with disposable inner cannula, Velcro ties, full pilot balloon, chest tube container full, no bubbling, chest tube dressing dry and intact. Need to (prompt) assess for crepitace then tell them there is none.

Interventions to perform: change out chest tube container, suction trach, trach care with disposable and Velcro ties

Problems to solve: full container, clot in CT tubing (milk), coughing and mucus from trach, reassessment after suctioning

Vital Sim Settings: R rhonchi at 6, L decreased sounds at 3, RR 24 BP 130/70 HR 90, normal rhythm. Change rhonchi to clear after suctioning

2. 69 yo male with past medical history of CAD, COPD, Afib, is admitted for dehydration secondary to gastritis. NS @ $125 / \mathrm{hr}$ in the R CVL. On RA c/o SOB developing this AM

Assessment finding students should note: Rhonchi - rales(crackles) at the bases, cough, increased RR SaO2 80. CVL dressing needs changed (note expired date), moist mucus, good turgor, adequate urine light yellow.

Interventions to perform: note FVE and decrease IV rate to TKO (KVO), start $\mathrm{O} 2$ with $\mathrm{NC}, \mathrm{F} / \mathrm{U}$ assessment $\mathrm{SaO} 2$ is 84 - change $\mathrm{O} 2$ to Venti mask or $\mathrm{NRB}$, change $\mathrm{CVL}$ dressing and caps .

Problems to Solve: needing additional $\mathrm{O} 2$ and reassessment, calling MD (discuss what to say, how to say it and what you want to get for orders i.e. lasix, decreased IV rate, maybe CXR.

Vital Sim Settings: BP 170/90, HR 122 and bounding, R Rhonchi (3), L crackles (8) RR 34, intermittent cough 
3. 36 yo male S/P MVA w/ Fx ribs and L chest tube for pneumothorax. Orally intubated with O2@40\% via T-piece. $23 \mathrm{~cm}$ at the lip line.

Assessment findings students should note: Breath sounds absent on L, CT dressing saturated and loose, gentle intermittent bubbling in $\mathrm{CT}$, rhonchi on $\mathrm{R}$, check pilot balloon (OK), measure ET tube at lip line, noting needed equipment (ambu bag and CT clamps)

Interventions to perform: Change CT dressing, suction, move ET tube to other side of mouth, measure drainage (explain how), using an Ambu bag to hyperoxygenate during suctioning. Suctioning.

Problems to solve: CT falls over spilling drainage into other chambers - how to measure output now, missing CT tube clamps

Vital Sim Settings: R rhonchi (5), L absent, RR 32, BP 140/80 HR 90

4. 22yp S/P Closed Head Injury in a persistent vegetative state w/ trach collar @ 35\% FiO2, G tube feed at $70 \mathrm{cc} / \mathrm{hr}$, HOB at 30 degrees.

Assessment findings students should note: Do a complete neuro check including GCS. When it is assessed you can tell students that every time they touch patient they decorticate (flexion), they are looking around with eyes open but not connecting, pupils at $3 \mathrm{~mm}$ and sluggish, positive babinski, pilot balloon is flat, mucus around trach with occasional moan. O2 at wrong setting $40 \%$,

Interventions to perform: suction with ballard and clean tube, trach care with permanent cannula and twill ties., attempt to fill pilot balloon using stethoscope to listen for air leak and discover it has a leak (really does) and perform immediate interventions of raising HOB to prevent aspiration, checking orders for deflated cuff.

Problems to solve: cuff with air leak, blood tinged sputum with suctioning

Vital Sim Settings: RR 34, Rhonchi bilaterally, cough when they are assessing lungs, BP 150/90 HR 88 
Appendix C

NURS 216 HPS Scenarios

$2007 / 2008$ 


\section{West Virginia University Parkersburg \\ Health Sciences Division \\ Department of Nursing \\ Nursing 216 HPS Scenarios 2007-2008 \\ Debbie Murphy, MSN, RN}

1. 40 yo female w/ L lobectomy for Ca. L chest tube to $20 \mathrm{~cm}$ suction, trach to T-piece with $40 \% \mathrm{O} 2$.

2. 69 yo male with past medical history of CAD, COPD, Afib, is admitted for dehydration secondary to gastritis. NS @ $125 / \mathrm{hr}$ in the R CVL. On RA c/o SOB developing this AM

3. 36 yo male S/P MVA w/ Fx ribs and L chest tube for pneumothorax. Orally intubated with O2@40\% via T-piece. $23 \mathrm{~cm}$ at the lip line.

4. 22yp S/P Closed Head Injury in a persistent vegetative state w/ trach collar @ 35\% FiO2, G tube feed at $70 \mathrm{cc} / \mathrm{hr}, \mathrm{HOB}$ at 30 degrees.

5. Cardiac Arrest - Basic Code

6. Hypoglycemia / DKA

7. PE secondary to DVT 


\section{Appendix D}

Orientation to Simulation Materials 


\section{ORIENTATION TO SIMULATION}

Welcome to the WVU Parkersburg Simulation Lab. Our goal over the next four semesters is to provide you with simulated clinical experiences to enhance classroom and clinical learning. Our goals are to reinforce your knowledge base with experiential learning, improve your skills in managing high risk low incidence situations, help you to communicate effectively with a team, and to learn to problem-solve and make clinical judgments in a safe environment.

You will be doing cases in a simulated environment using high fidelity mannequins. We attempt to make the situations appear, sound, smell and feel as much like the actual clinical setting as we can make it. The patients you encounter will be different individuals although they look very similar because they are sophisticated computer controlled mannequins. Each patient has a name, age, medical and social history and a personality. Most patients have a voice and will be able to converse with you in a normal fashion although the mouth does not move when the patient speaks. Each patient has a chart with pertinent information that you would find in the actual clinical setting.

Structure: Each simulated clinical experience will be structured the same. Your group will be divided into 2 equal groups. The first group will go through the scenario while the second group watches from an adjacent room. We will then switch places and run the scenario again. The observing group will be expected to watch closely and take good notes on how the group performed and how they might do things differently so that they can give good feedback during the debriefing session. Each person in the group will have a specific role to play. Possible roles and responsibilities of the role include:

PRIMARY NURSE - Conduct primary assessment including vital signs, conduct a situational analysis, consult team, make decisions, run a code and DELEGATE clearly. Ensure that the team is progressing efficiently through the scenario and make adjustments as necessary.

SECONDARY NURSE - Assist the primary nurse with assessment, situational analysis, consultation and decision-making. Assist the treatment nurse as needed. Ventilations or compressions during a code.

TREATMENT NURSE - Complete any interventions that are needed such as application of oxygen, changing dressings, positioning, foley catheter insertion, suctioning, etc. Do what ever is delegated by the primary nurse. Make suggestions to the primary nurse. Ventilations or compressions during a code.

DOCUMENTATION/COMMUNICATIONS - Check chart for results, notes, history, orders, etc. and communicate information needed to the team. Document assessment data, medications given or care provided. Help develop a problem list. Call anyone that needs to be called using SBAR when appropriate. Act as recorder in a code. Make suggestions to the primary nurse.

Maintain whiteboard and keep team on track as necessary.

MEDICATION - Administer any medications that are ordered or needed. Initiate, monitor, change/adjust, trouble shoot any IV ${ }^{e e}$. May seek assistance from the treatment nurse or secondary nurse. Document appropriately. 
TREATMENT NURSE 2 - When there is a group of 6 a second treatment nurse will be the $6^{\text {th }}$ role.

After both groups have completed the scenario, we will move to another room and conduct a "Debriefing Session" where we reflect on what happened during the scenario, clarify any information, and discuss problem-solving during the situation. This session may use video/audio recordings of the simulation to review what occurred.

Policies: It is important that you arrive on time, in appropriate uniform and with your prep sheet completed. Place all your belongings in a locker including your lab coat and come into the lab ready to go. Although simulation is not a graded activity you can receive a clinical unsatisfactory if you do not comply with the above. We do not grade simulation because it is an opportunity for you to step out of your comfort zone, try new things, and feel safe - that you will not hurt a patient or negatively impact your clinical evaluation. It is a learning environment where mistakes are expected. You will learn from those mistakes. We are not judging you but we will provide meaningful feedback on your performance to enhance your learning.

Simulation absence policy: Attendance is expected at all assigned simulations. Simulation dates and times will be assigned to the student prior to the start of the semester. No alterations in schedule will be permitted. It is the expectation that the student attends his/her scheduled simulation time. Failure to do so will result in an absence. Any missed simulation must be made-up. A student who misses a simulation must notify the Simulation Coordinator or Lab Manager prior to the simulation sessions. Failure to do so will result in an unsatisfactory clinical day. Do not call the secretary or your course or clinical instructors. Excessive absences from simulation, which is more than one missed simulation, will result in the student repeating the course. If a second simulation is missed, the student will be administratively withdrawn and will be required to repeat the course.

General issues with using mannequins: (There are individual guidelines for each mannequin in this eCampus folder) Each scenario will begin with a situation that you are given. You will have time to review the situation and discuss with your team possible approaches. You will then enter the room and begin to assess your patient and the situation. You will review the chart for history, orders, lab and diagnostic data etc. With the exception of the infants in N213 you may talk directly to the patient and he or she will answer you. You will conduct an assessment as appropriate to the situation. You will conduct the assessment just as you would on a real patient. We try not to "pretend". If the data can be acquired from the mannequin, you will need to get it and document it, i.e. pulse, breath sounds, pupil size. If the data you require can not be obtained from the mannequin, i.e. skin temperature and moisture, pupil reaction, muscle movements etc., then you need to go through the motions of assessing that parameter and the information will be given to you by intercom or by faculty in the room. All mannequins have a compressor that cycles on and off. They are annoyingly loud and will make it difficult to hear sounds and vital signs. You will just need to wait until it turns off and reassess. Please see the individual mannequin orientation as assessment capabilities will vary depending on the mannequin you are using. You will carry out all orders, medications, and treatments just as you would on a real patient. Do not "simulate" or "pretend". We try not to reuse any supplies (unlike campus labs). In most instances you will obtain new kits, supplies, etc. and open and use them appropriately. We have two simulation supply carts and a medication card. Look for what you need in the room or on these three carts.

Exception: NEVER use betadine on a mannequin! You may use alcohol or chlorhexidine. NEVER use standard lubricant on a mannequin - ONLY silicone spray which will be labeled "water soluable lubricant" 
Phone calls: If you need to call someone - lab or radiology for results, doctors for orders, operators for codes - you have a real working phone in the room. The phone numbers are listed on the wall. A faculty member will answer the phone and act as the appropriate person or department and provide you with what you require. If the phone should ring, answer it. It will be either a faculty member calling you within the context of the scenario or a wrong number. Inform the caller if it is a wrong number and hang up. These phones are unpublished numbers.

NEVER DIAL $O$ or the Operator to call a code!! Use the number on the phone list on the wall.

Medications: All medications at WVUP Hospital are prepared in appropriate containers and are labeled with the generic name, dosage and strength. Medications are in a cart or bins with the patient "s name. Medications come in all routes and will be administered as appropriate with the exception of PO medications. You will "simulate" administering them but do not actually put the medication or water into the mannequin "s mouth or airway. Topical, rectal, parenteral and intravenous drugs are given in the normal manner. (see comments with each mannequin)

Monitors and Resusitation: If a patient requires cardiac monitoring, the electrodes can either be snapped into place on the pegs on the mannequin "s chest or actual electrodes can be attached in appropriate locations (over the pegs). Once the electrodes have been applied the rhythm will appear on the physiological monitor in the patients room. Monitor displays will vary depending on the level you are in the program. At a minimum you will have a heart rate and an SpO2 (pulse ox) when you apply the probe to the mannequin "es finger. Oxygen delivery devices, bag-valve-mask devices are available on the supply cart. When needed there is a crash cart with a defibrillator. You will apply the gel pads as appropriate and go through the motions of charging, clearing and discharging the paddles on the chest but the unit WILL NOT be turned on. When you shock, the patient will not move because there is no skeletal musculature. The "quick-look" feature of the defibrillator will not work. You need to always look at the physiologic monitor instead of the defibrillator screen to interpret the rhythm. We will not intubate the patient.

Stress: All participants in simulation feel some degree of stress or anxiety from the experience. The combination of responding to medical crises, practicing in an unfamiliar setting, the uncertainty of the simulation itself, being observed by peers and faculty, watching oneself on videotape among colleagues will all produce some anxiety for most people. You are not unusual if you experience this. A modicum of this response occurs regardless of one ${ }^{\text {ee }}$ experience or background. Generally this degree of stress is harmless and probably contributes to increased learning in most people. Most students have a positive feeling about the learning that takes place in simulation despite the anxiety that it produces. In rare instances, however, a participant is truly upset by the experience, their performance, or how others treated them. If you feel that you are upset by the experience and your reaction persists you are welcome to contact one of the faculty and talk about the situation. We want this to be a positive learning experience for everyone.

Again, welcome. We hope you have a successful, productive, fun semester of simulation. 
Appendix E

Simulation Prep Worksheets 
Nursing 111

Simulation Lab Preparation Cycle 4

Andrews

1. List signs and symptoms of fluid volume deficit.

2. What electrolyte imbalances occur with dehydration? What signs and symptoms would you see with each?

3. What acid-base disturbance can occur? What signs and symptoms would you see?

4. How do you treat electrolyte imbalances from dehydration? How could the patient be rehydrated? Are there any dangers in treating fluid and electrolyte imbalances in dehydration?

5. What fluids are used to correct fluid volume deficit from dehydration?

6. How will you know if the treatment is effective - list specific parameters or goals.

7. What are the manifestations of heat exhaustion?

8. How can a patient with heat exhaustion be cooled? 
Nursing 112

Simulation Lab Preparation Cycle 7

Lowers

1. List the assessments that are important for a patient who has had an abdominal hysterectomy? Put them in order of priority. List general post-op and specific hysterectomy assessment parameters separately.

2. What complications can occur following an abdominal hysterectomy? List the signs and symptoms for each complication and interventions for prevention of each.

\begin{tabular}{|l|l|l|}
\hline Complication & Signs and Symptoms & \\
\hline & & \\
& & \\
& & \\
& & \\
\end{tabular}

3. Discuss the relationship between $\mathrm{CBC}$, bleeding and decreased urine output, fatigue, exertional dyspnea and dizziness. What is the physiology of this interrelationship.

4. Describe the procedure for administering an IV push dose of medicine. How would the procedure differ if you were giving it in a running IV versus an IVL versus a central line?

5. List the IV administration information (rate, dilution, compatibilities) and relevant patient data to assess for the following drugs: Zophran, Dilaudid 
Nursing 213

Simulation Lab Preparation Cycle 7

Callahan

1. List the signs and symptoms of bipolar disorder. Include and differentiate between depressive, manic and hypomanic states.

2. Discuss lithium as a treatment for bipolar disorder. Include relevant patient data, side effects, toxicity, and drug interactions.

3. What are the normal and toxic blood levels of lithium and discuss the management of toxicity.

4. Develop a teaching plan for a patient with bipolar disorder being treated with lithium. Include disease process and management and drug therapy in the teaching plan. 
Nursing 216

Simulation Lab Preparation Cycle 2

Evans

1. Describe the management of chest pain - "chest pain protocol".

2. What complications can occur with an inferior wall MI?

3. Describe the EKG changes that occur with ischemia and infarction - distinguish between the two.

4. What are the characteristics of an atrial fibrillation rhythm?

5. What complications can occur with atrial fibrillation? What signs and symptoms would you see if the complications are occurring? (make a little chart)

6. Review med sheet information for nitroglycerine, metaprolol, and morphine

7. Review location of EKG electrodes. 
Appendix F

Simulation Curriculum with Objectives 


\section{West Virginia University Parkersburg \\ Health Sciences Division \\ Department of Nursing \\ Nursing 111 \\ Simulation Curriculum}

Orientation: Simulation and Mannequin Orientation, SBAR Review, 60 Second Assessment review

Cycle 1: $\quad$ Bed bath, application of O2, notification of HCP

Scenario: $\quad$ AM Care with activity intolerance - Lois Hodgkins Situational Awareness \#1

Cycle 2: $\quad$ NG skills and medication administration

Scenario: $\quad$ N\&V NG insertion - Skylar Hanson

Situational Awareness \#2

Cycle 3: $\quad$ Responding to an acute situation, medication administration

Scenario: $\quad$ CNS depression from opiod intoxication - Doris Bowman

Cycle 4: (Review flush and bolus, first week Monday and Thursday - no foley Hang an IV fluid bolus, saline flush, interpret labs, application of cold, foley

Scenario: Dehydration with fluid and electrolyte imbalance - Matt Anderson

Cycle 5: $\quad$ Restraint protocols, dealing with upset SO, IM meds, saline flush, ABG interpretation, foley insertion for incontinence

Scenario: Anxiety with restraints - Charles Alden 


\section{West Virginia University Parkersburg Health Sciences Division Department of Nursing Nursing 112 \\ Simulation Curriculum}

Cycle 1

Scenario: $\quad$ Pneumonia - Reaction to ABX - Kenneth Bronson

Cycle 2

Scenario: Chemo administration with N\&V, hypovolemia - George Riviera

Cycle 3

Scenario: $\quad$ Post-op Hemmorrhage - John Hoover

Cycle 4

Scenario: $\quad$ CHF exacerbation - Robert Gaines

Cycle 5

Scenario: $\quad$ Post-op Hip ORIF - transfusion reaction - Lloyd Bennett

Cycle 6

Scenario: Hypoglycemia / DKA - Tim Moore

Cycle 7

Scenario: $\quad$ Abdominal hysterectomy with FVD, bleeding - Amanda Lowers 


\section{West Virginia University Parkersburg Health Sciences Division Department of Nursing Nursing 213 \\ Simulation Curriculum}

Cycle 1

Scenario: Meti: Acetaminophen poisoning - Tommy Turner

Cycle 2

Scenario: Meti : Asthma attack in Pediatric Patient - Keven McGraw

Cycle 3

Scenario: $\quad$ Meti : First trimester with hyperemesis - Susan Roper

Cycle 4

Scenario: Meti : Post-partum hemorrhage - Rochelle and Baby Carter

Cycle 5

Scenario: $\quad$ Meti : Myelomeningocele - Patrick Dugan

Cycle 6

Scenario: $\quad$ Meti : Abruptio Placentae - Carrie Fisher

Cycle 7

Scenario: Meti : Bipolar Disorder with Lithium Toxicity - Alice Callahan 


\section{West Virginia University Parkersburg \\ Health Sciences Division \\ Department of Nursing \\ Nursing 216 \\ Simulation Curriculum}

Cycle 1

Scenario: $\quad$ Suctioning and Trachea Care with Hypoxia - John Byers

Cycle 2

Scenario: $\quad$ Chest Pain Protocol - Sam Evans

Cycle 3

Scenario: Sepsis, Septic Shock - Carl Bramson

Cycle 4

Scenarios: ARF - Ray Carlson

Cycle 5

Scenario: $\quad$ Spinal Cord Injury - Connor Lund

Cycle 6

Scenario: $\quad$ Meti: Brain Attack with Thrombolytic Therapy - Brian Bush

Cycle 7

Scenario: $\quad$ Cardiac Arrest - Basic Code - Henry Lopez 


\section{Appendix G}

Institutional Review Board Exempt Letter 


\title{
WestVirginiaUniversity.
}

\author{
Office of Research Compliance
}

\section{IRB Protocol-Exemption}

To: $\quad$ Breault, Rick

From: $\quad$ WVU Office of Research Compliance

Date: $\quad$ Tuesday, June 12, 2012

Subject: Exemption Acknowledgement

Tracking \#: $\mathrm{H}-24103$

Title: Relationship between fidelity and dose of human patient simulation (HPS), critical thinking abilities, and knowledge in an associate degree nursing program: An exploratory study

The above-referenced study was reviewed by the West Virginia University Institutional Review Board (IRB) and was granted exemption in accordance with 45 CFR 46.101(1).

This protocol was reviewed using the following:

This research study was granted an exemption in accordance withResearch on normal educational practices [45 CFR 46.101(1)].Exemption checklist

The following documents have been acknowledged for use in this study and are available in the BRAAN system:

Thank you.
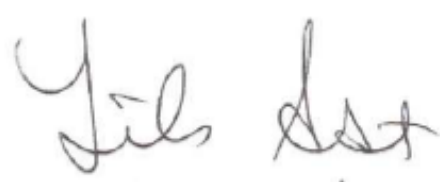

Board Designee: Ast, Lilo

Letter Sent By: Ast, Lilo, 6/12/2012 10:29 AM

Once you begin your human subject research, the following regulations apply:

1. Any modifications to the study protocol must be reviewed and acknowledged by the IRB prior to implementation.

2. You may not use a modified form until it has been acknowledged by the IRB. 


\section{Appendix $\mathrm{H}$}

West Virginia University at Parkersburg Approval 


\section{We Parkersburg \\ West VrginiaUniversity.}

OFFICE OF ACADEMIC AFFAIRS

Rhonda Tracy, Ph.D., Senior Vice President, Academic Affairs

\section{MEMORANDUM}

TO:

Rose Beebe

FROM:

RT

RE:

Rhonda Tracy, Senior Vice President for Academic Affairs

DATE:

Dissertation Research

CC:

May 23, 2012

Jeremy Starkey

You have permission to utilize data from the Health Sciences database and demographic information for your identified sample group as part of your dissertation research project. Information approved for your use includes the following: a) nursing student admission data; b) ACT scores; c) GPA; d) admission exam score; e) CT scores, and f) the exit score and critical thinking subscores, Student anonymity must be maintained in your research.

Best of luck as you continue your progress toward completion of your doctoral degree.

Kind regards.

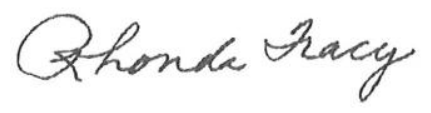


Appendix I

Elsevier/HESI Nurse Researcher Agreement 

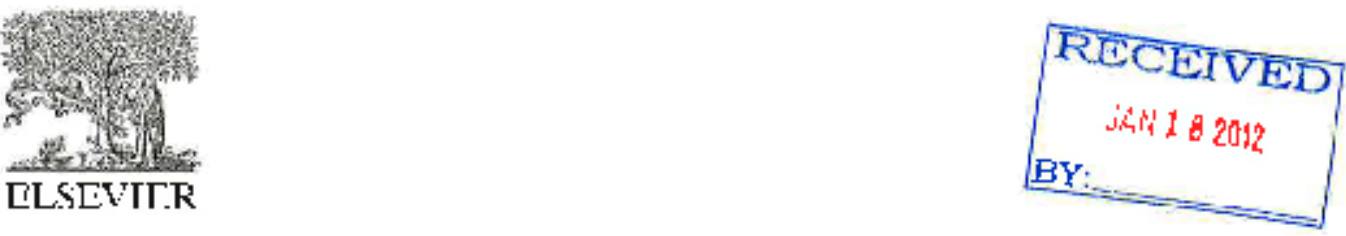

Nurse Resegrcler Agreement to part|c|pate In ElsevleriHE\$1 Educatlongl Rege日rch Projects

Thank you tor ardeelng to partlc|pate In Clsevle.3HC

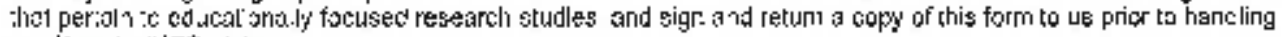
any E.soverllES data.

:1: All data recelved by ire nurse researcher for anaysls must the ma atalned in a secure ocation for ihe

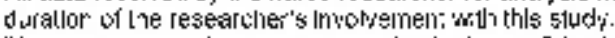

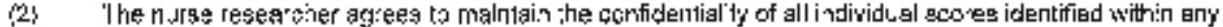
dste Eu:ו1וne $y$ dacument

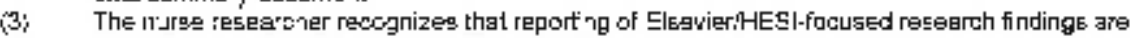

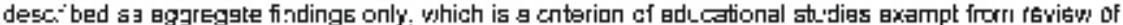

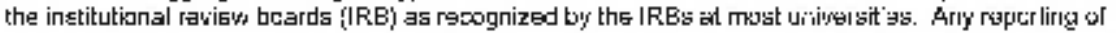

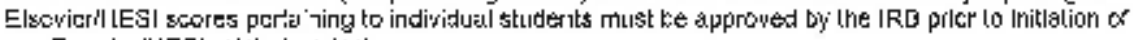
a'y E scricr'HESI-related projctst.

(4) Unloss IRE approval ollichuse alctates, onco the final aralys's of tre data s complate, the nurse

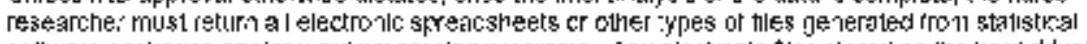
scrtware oackages anc'cr wcrd precessing programs. Any electionic tiles storec on 1re hand drive(s) of

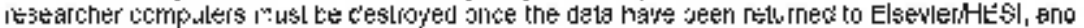

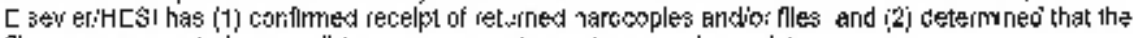
flles are urcornoted, accesslb:e on our ccmputer systems, ard ccmp'ete.

(5) If the nurse esearcher does not ineet toeir dissemlration plan, a report nay ke written for fisgem nation

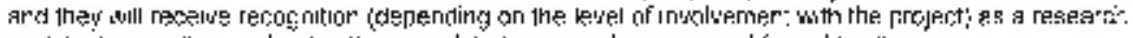
assistent, co-author. or lead author on related menuscriprs prepered for pubisation.

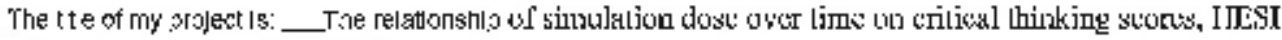
exil exdm: SL'utes, and NCT.TXX piss rales.

This agreersent g for the t merialne 1,2012 Mbnthi'Yegri to 2 i20'2

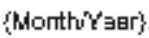

Wly c eseminaticr pign is _ D esertation defenes by 12,2012 (Pont!'Yegrị.

For any gues.icrs. sontact:

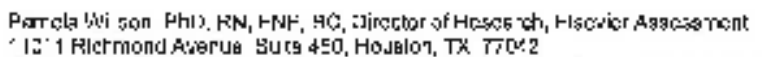

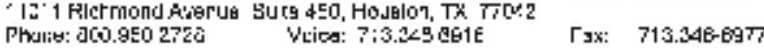

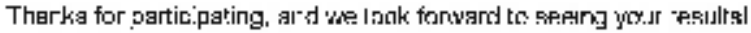

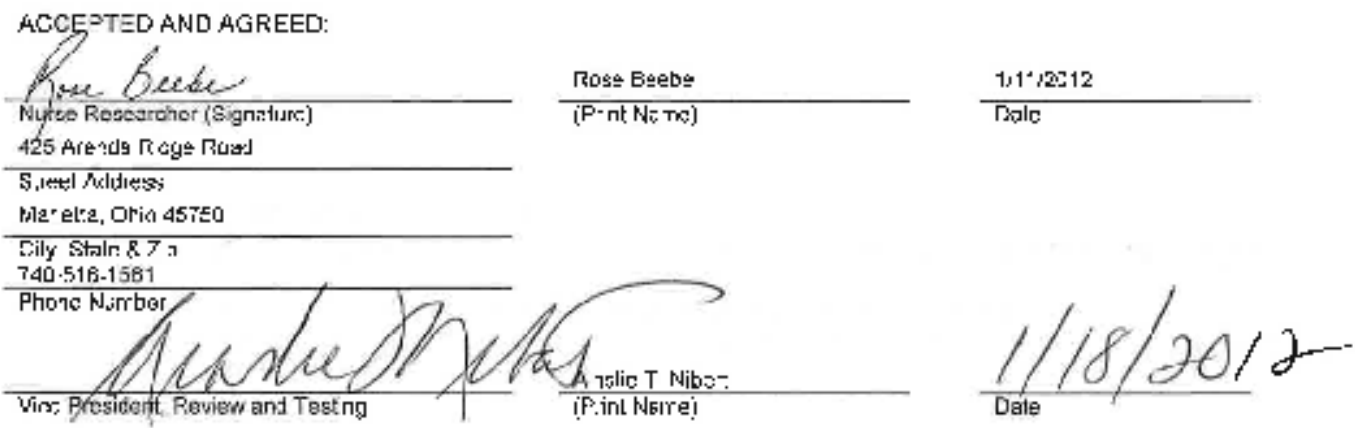

